NIST Technical Note 1816

\title{
Performance Criteria for an ASTM XRF STANDARD TEST METHOD FOR HYdRAUliC CEMENTS: INTER-LABORATORY STUDY ON CEMENTS A AND B
}

Paul Stutzman

Alan Heckert

http://dx.doi.org/10.6028/NIST.TN.1816

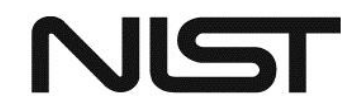

National Institute of Standards and Technology U.S. Department of Commerce 


\section{Performance Criteria FOR AN ASTM XRF STANDARD TEST METHOD FOR CHEMICAL ANALYSIS OF Hydraulic CEMENTS: INTER-LABORATORY STUDY ON CEMENTS A AND B}

Paul Stutzman Materials and Structural Systems Engineering Laboratory

Alan Heckert Statistical Engineering Information Technology Laboratory

http://dx.doi.org/10.6028/NIST.TN.1816

October 2013

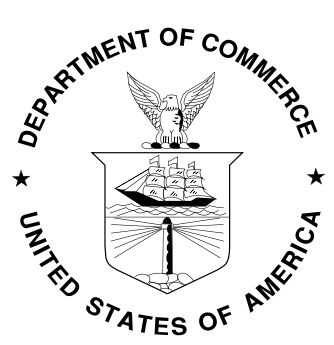

U.S. Department of Commerce Penny Pritzker, Secretary

National Institute of Standards and Technology Patrick D. Gallagher, Under Secretary of Commerce for Standards and Technology and Director 
Certain commercial entities, equipment, or materials may be identified in this document in order to describe an experimental procedure or concept adequately. Such identification is not intended to imply recommendation or endorsement by the National Institute of Standards and Technology, nor is it intended to imply that the entities, materials, or equipment are necessarily the best available for the purpose.

National Institute of Standards and Technology Technical Note 1816 Natl. Inst. Stand. Technol. Tech. Note 1816, 199 pages, October 2013 CODEN: NTNOEF http://dx.doi.org/10.6028/NIST.TN.1816 


\begin{abstract}
Bulk oxide determinations from a pair of portland cements provide the basis for calculating the precision and accuracy values for X-ray fluorescence (XRF) analysis for both the fused glass bead and the pressed powder sample preparation methods. The measurements were collected through an interlaboratory study using two cements (Cement A and Cement B), and the two sample preparation methods were divided (roughly evenly) among the laboratories. Each of the approximately 45 participating laboratories provided six replicate measurements, analyzed in duplicate, for each of the two separate portland cements. Each laboratory reported an estimate for the mass fraction of eleven analytes: $\mathrm{CaO}, \mathrm{SiO}_{2}, \mathrm{Al}_{2} \mathrm{O}_{3}$, $\mathrm{Fe}_{2} \mathrm{O}_{3}, \mathrm{SO}_{3}, \mathrm{MgO}, \mathrm{Na}_{2} \mathrm{O}, \mathrm{K}_{2} \mathrm{O}, \mathrm{TiO}_{2}, \mathrm{P}_{2} \mathrm{O}_{5}$, and $\mathrm{Cl}$. Chemical data using traditional chemical analyses (the Reference Methods) from the Cement and Concrete Reference Laboratory (CCRL) proficiency test program were included for comparison to the XRF results. Precision measures for within- and between-laboratory performance are presented as $1-\sigma$ and $95 \%$ limits (ASTM d2s). Accuracy criteria are based upon a two-sided $95 \%$ prediction interval for the mean of two test results, defining the range of values one might expect for each analyte relative to a certified value of a reference material.
\end{abstract}

Keywords: accuracy; hydraulic cement; precision; qualification; X-ray fluorescence 


\section{Table of Contents}

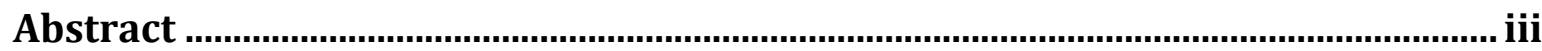

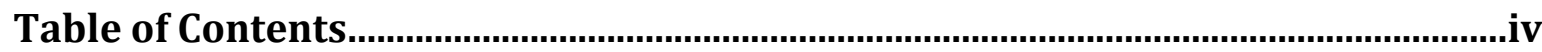

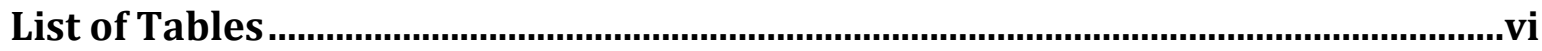

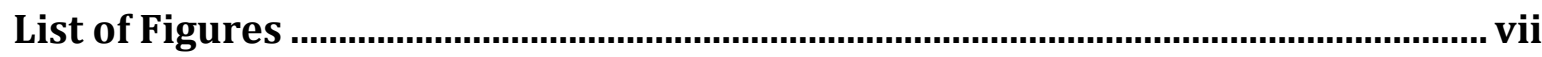

Introduction ........................................................................................................ 1

Development of a Test Method for XRF Analysis of Hydraulic Cements........................... 3

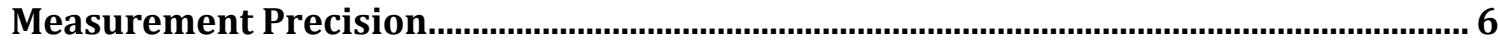

Measurement Accuracy and Method Bias......................................................................... 6

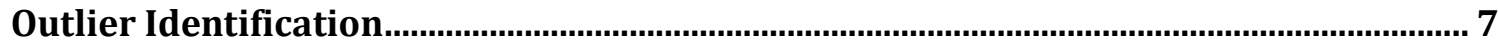

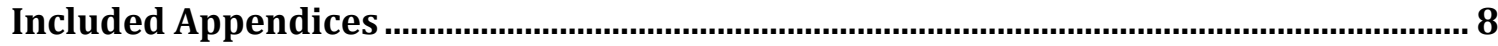

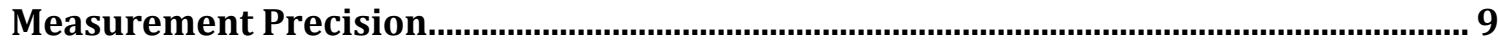

Prediction Intervals ......................................................................................................12

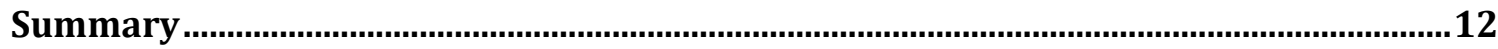

Appendix A. Individual Oxide Analysis.................................................................13

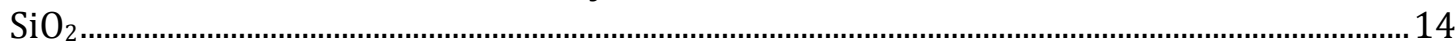

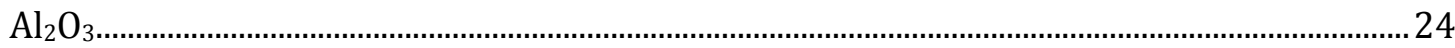

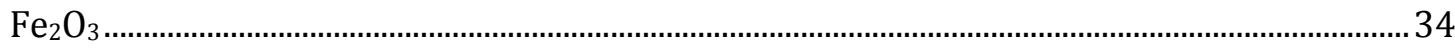

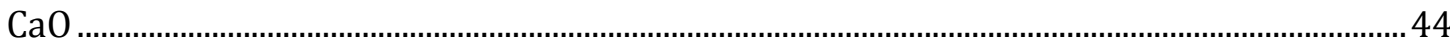

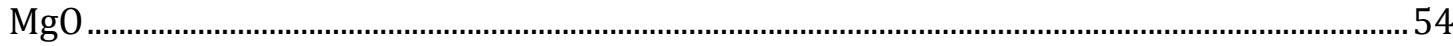

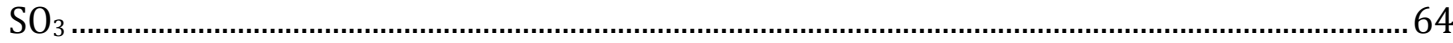

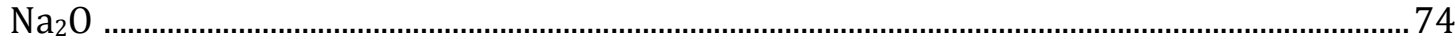

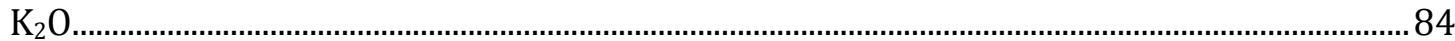

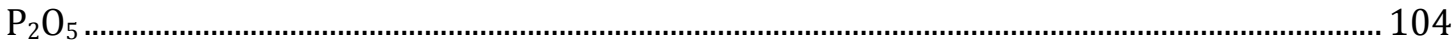

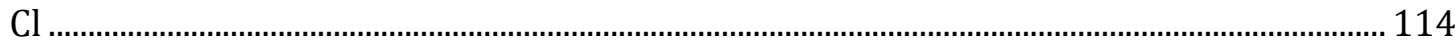

Appendix B. Request for Participants Letter........................................................ 121

Appendix C. XRF Glass Summary by Material and Replicate. ............................. 124

Appendix D. XRF Powder Summary by Material and Replicate............................ 126

Appendix E. Raw Data by Analyte............................................................................. 128

$\mathrm{SiO}_{2}$

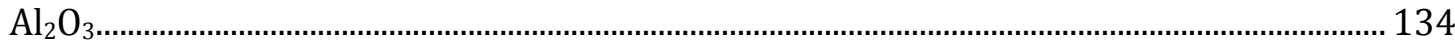

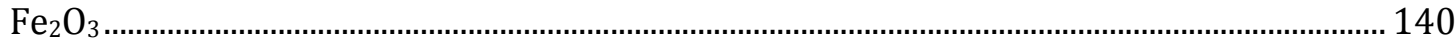

$\mathrm{CaO}$

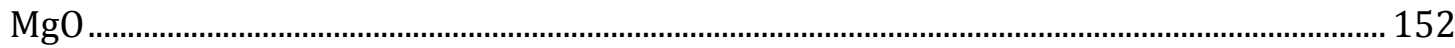

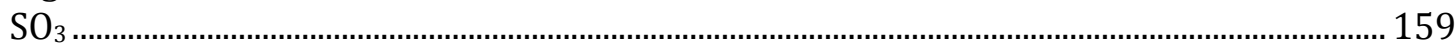

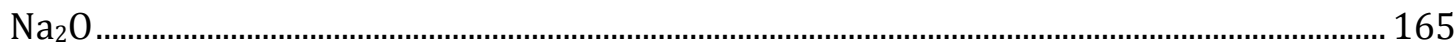

$\mathrm{K}_{2} \mathrm{O}$

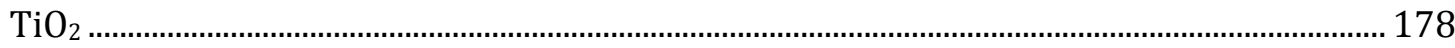

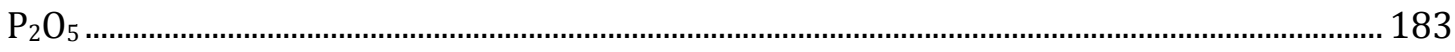

$\mathrm{Cl}$

Acknowledgements ........................................................................................... 191 


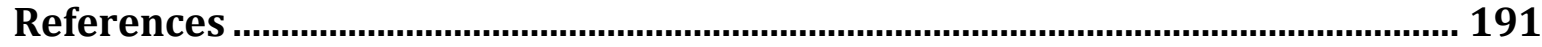




\section{List of Tables}

Table 1 Pooled Results for XRF-Glass, XRF-Powder and Combined Powder and Glass Methods, when the results are expressed as a percent. The values include the $1 \sigma$ within-laboratory $(\mathrm{Sr})$, the $1 \sigma$ between-laboratory (sR), and the ASTM d2s values for the $95 \%$ limit on the difference between two test results (r) and (R).

Table $295 \%$ Prediction Interval designed to bracket values of a mean of $k=2$ measurements. The mean result should differ from the known value of the certified reference material by no more than the value shown for each analyte.

Table 3 Precision Statistics $\mathrm{SiO}_{2} \mathrm{XRF}$, Cements A B

Table 4 Precision Statistics $\mathrm{Al}_{2} \mathrm{O}_{3}$ by XRF glass and powder preparations for replicates 1 and 2

Table 5 Precision Statistics $\mathrm{Fe}_{2} \mathrm{O}_{3}$ by XRF glass and powder preparations for replicates 1 and 2

Table 6 Precision Statistics $\mathrm{CaO}$ by XRF glass and powder preparations for replicates 1 and 2

Table 7 Precision Statistics $\mathrm{CaO}$ by XRF glass and powder preparations for replicates 1 and 2.

Table 8 Precision Statistics SO3 by XRF glass and powder preparations for replicates 1 and 2 73

Table 9 Precision Statistics $\mathrm{Na}_{2} \mathrm{O}$ by XRF glass and powder preparations for replicates 1 and 2 .

Table 10 Precision Statistics $\mathrm{K}_{2} \mathrm{O}$ by XRF glass and powder preparations for replicates 1 and 2 .

Table 11 Precision Statistics $\mathrm{TiO}_{2}$ by XRF glass and powder preparations for replicates 1 and 2 .

Table 12 Precision Statistics $\mathrm{P}_{2} \mathrm{O}_{5}$ by XRF glass and powder preparations for replicates 1 and 2 . 


\section{List of Figures}

Figure 1. ASTM C114 performance criteria for the chemical analysis of portland cements. From a letter by W.C. Hanna to the Working Committee on Methods of Chemical Analysis, June 12, 1946.

Figure 2 Reference and instrumental methods popularity from CCRL proficiency test data shows that over $80 \%$ of the labs use X-ray methods for bulk chemical analysis by either energy- or wavelength-dispersive analysis, using either powder or glass specimens.

Figure 3 Calculated values for the determination of within and between lab precision....... 5 Figure 4 Scatter plot for $\mathrm{CaO}, \mathrm{XRF}$-powder preparation as boxes with the "o" symbol noting excluded data due to exceeding the $\mathrm{h}$ or $\mathrm{k}$ statistics. 8

Figure $5 \mathrm{Lab}$ means and standard deviations for $\mathrm{CaO}$, powder preparation for cement $\mathrm{A}$ (Material 1) and cement B (Material 2).

Figure $6 \mathrm{~h}$ and k statistic plots illustrate the within- $(k)$ and between-laboratory $(h)$ precision and are useful in identifying unusual results that may be considered outliers. The dashed line marks the limits for each statistic.

Figure 7 Box plots for $\mathrm{SiO}_{2}$ for XRF glass and powder, and reference methods.............. 14 Figure $8 \mathrm{SiO}_{2}$ precision statistics by method with bar chart comparing results to current and past ASTM C114 limits and previous studies on chemical analysis precision as 1sigma, between lab $\left(\mathrm{S}_{\mathrm{R}}\right)$.

Figure 9 Box plots for $\mathrm{Al}_{2} \mathrm{O}_{3}$ XRF glass and powder, and reference methods.................. 24 Figure $10 \mathrm{Al}_{2} \mathrm{O}_{2}$ precision statistics by method with bar chart comparing results to current and past ASTM C114 limits and previous studies on chemical analysis precision as 1sigma, between lab $\left(\mathrm{S}_{\mathrm{R}}\right)$

Figure 11 Box plots for $\mathrm{Fe}_{2} \mathrm{O}_{3}$ XRF glass and powder, and reference methods .. 34

Figure $12 \mathrm{Fe}_{2} \mathrm{O}_{3}$ precision statistics by method with bar chart comparing results to current and past ASTM C114 limits and previous studies on chemical analysis precision as 1sigma, between lab $\left(\mathrm{S}_{\mathrm{R}}\right)$

Figure 13 Box plots for $\mathrm{CaO}$ XRF glass and powder, and reference methods.

Figure $14 \mathrm{CaO}$ precision statistics by method with bar chart comparing results to current and past ASTM C114 limits and previous studies on chemical analysis precision as 1sigma, between lab $\left(\mathrm{S}_{\mathrm{R}}\right)$.

Figure 15 Box plots for MgO XRF glass and powder, and reference methods.

Figure $16 \mathrm{MgO}$ precision statistics by method with bar chart comparing results to current and past ASTM C114 limits and previous studies on chemical analysis precision as 1sigma, between lab $\left(\mathrm{S}_{\mathrm{R}}\right)$.

Figure 17 Box plots for $\mathrm{SO}_{3}$ XRF glass and powder, and reference methods.

Figure $18 \mathrm{SO}_{3}$ precision statistics by method with bar chart comparing results to current and past ASTM C114 limits and previous studies on chemical analysis precision as 1sigma, between lab $\left(\mathrm{S}_{\mathrm{R}}\right)$.

Figure 19 Box plots for $\mathrm{Na}_{2} \mathrm{O}$ XRF glass and powder, and reference methods. 74 Figure $20 \mathrm{Na}_{2} \mathrm{O}$ precision statistics by method with bar chart comparing results to current and past ASTM C114 limits and previous studies on chemical analysis precision as 1sigma, between lab $\left(\mathrm{S}_{\mathrm{R}}\right)$.....

Figure 21 Box plots for $\mathrm{K}_{2} \mathrm{O}$ XRF glass and powder, and reference methods. 
Figure $22 \mathrm{~K}_{2} \mathrm{O}$ precision statistics by method with bar chart comparing results to current and past ASTM C114 limits and previous studies on chemical analysis precision as 1-

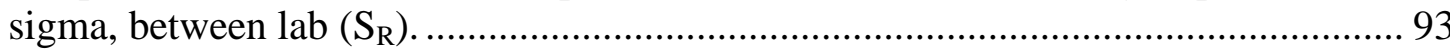

Figure 23 Box plots for $\mathrm{TiO}_{2}$ XRF glass and powder, and reference methods .................. 94 Figure $24 \mathrm{TiO}_{2}$ precision statistics by method with bar chart comparing results to current and past ASTM C114 limits and previous studies on chemical analysis precision as 1sigma, between lab $\left(\mathrm{S}_{\mathrm{R}}\right)$.

Figure 25 Box plots for $\mathrm{P}_{2} \mathrm{O}_{5}$ XRF glass and powder, and reference methods. ................ 104 Figure $26 \mathrm{P}_{2} \mathrm{O}_{5}$ precision statistics by method with bar chart comparing results to current and past ASTM C114 limits and previous studies on chemical analysis precision as 1sigma, between lab $\left(S_{R}\right)$. 113

Figure 27 Box plots for $\mathrm{Cl}$ XRF glass and powder, and reference methods. 114 


\section{Introduction}

Chemical analysis of portland cement is used for process control in clinker and cement manufacture, for demonstration specification compliance, and for relating chemical properties to performance attributes. Reporting requirements for the chemical composition of portland cements in North America date back to the 1915 Joint Conference on Uniform Methods of Tests and Standard Specifications for Cement which involved the American Society of Civil Engineers, the American Society for Testing Materials, and the United States Government and included the following analytes: $\mathrm{SiO}_{2}, \mathrm{Fe}_{2} \mathrm{O}_{3}, \mathrm{Al}_{2} \mathrm{O}_{3}, \mathrm{CaO}, \mathrm{MgO}$, $\mathrm{SO}_{3}$, Ignition Loss, and Insoluble Residue [1].

In 1946, work on harmonizing the Federal and ASTM Cement Specifications resulted in the development of ASTM C150, Specification for Portland Cement within ASTM C1 on Cement. Standard Methods of Chemical Analysis of Portland Cement, ASTM C114-44 was also published and contained a performance-based qualification scheme [Figure 1] that is very similar to that used today where duplicate determinations on different days are made and the two results must be within the limit of permissive variation with their average accepted as the correct value [2]. Using reference cements to qualify, this process was repeated if the criteria were not met.

The 1960's through the 1970's saw a shift from classical analytical "wet" chemistry measurements (referred to as the reference and the alternate methods) to instrumental methods, including atomic absorption spectrometry, X-ray spectrometry, and a spectrophotometric/titrimetric scheme. Forrester et al. [3], Midgley [4], Harrison et al. [5], Aldridge et al. [6,7], Stutzman and Lane [8], EN 196-2.2 [9], and a National Cooperative Highway Research Program report [10] have investigated uncertainty in chemical analyses of portland cements, with the latter three being based upon standardized methods. ASTM Subcommittee C 1.23, Chemical Analysis, amended the precision requirements with accuracy requirements in 1977 by replacing the third column in Figure 1 with a maximum difference between the mean of two replicate determinations and the value of a certified reference material. This amended set of criteria is now Table 1 of ASTM C114 and is the basis of method qualification for instrumental methods other than the reference methods, for example, those by X-ray fluorescence (XRF) [2]. While the column one requirements originated in the 1946 edition of ASTM C114 and are based upon the reference methods, none of the qualification requirements have supporting data available. The development of a standard test for XRF analysis of hydraulic cements provides an opportunity to collect the data and calculate appropriate qualification values. 


\begin{tabular}{|c|c|c|}
\hline \multicolumn{3}{|c|}{ Yeximum Lim1ts of $\mathrm{p} \Rightarrow \mathrm{rm} 1 \mathrm{se1 \textrm {V }}$ Var1otion } \\
\hline Component & $\begin{array}{l}\text { Between } \\
\text { two results } \\
\end{array}$ & $\begin{array}{l}\text { Betwaen the } \\
\text { extreme values } \\
\text { in three results } \\
\end{array}$ \\
\hline $\mathrm{S} 11100 \mathrm{r}$ diox1de, $\mathrm{S}_{2} \mathrm{O}_{2}$ & 0.16 & 0.24 \\
\hline Aluminum oxide, $\mathrm{Al}_{2} \mathrm{O}_{3}$ & 0.20 & 0.30 \\
\hline Ferric oxtde, $\mathrm{Fe}_{2} \mathrm{O}_{3}$ & $\cdot 0.10$ & 0.15 \\
\hline Celclum oxide, $\mathrm{CaO}$ & 0.20 & 0.30 \\
\hline Hagnesium ox1de, " Mo & 0.16 & 0.24 \\
\hline Sulfur trioxide, $\mathrm{SO}_{3}$ & 0.10 & 0.15 \\
\hline Lose on $1 \mathrm{gm} 1 \mathrm{tion}$ & 0.10 & 0.15 \\
\hline Sodium oxide, $1 a_{2} O$ & 0.03 & 0.05 \\
\hline Potassium oxide, $\mathrm{K}_{2} \mathrm{O}$ & 0.03 & 0.05 \\
\hline Phosphorus ventoxide, $\mathrm{P}_{2} \mathrm{O}_{5}$ & 0.03 & 0.05 \\
\hline Zanagen1c oxidn, $\mathrm{Nn}_{2} \mathrm{O}_{3}$ & 0.03 & 0.05 \\
\hline Insoluble residue & 0.20 & 0.15 \\
\hline $\begin{array}{l}\text { Chloroform - soluble } \\
\text { orzanic substances }\end{array}$ & 0.004 & 0.006 \\
\hline Free calcium oxide & 0.20 & 0.30 \\
\hline 7eter - soluble alkal1 & 0.05 & 0.08 \\
\hline
\end{tabular}

Figure 1. ASTM C114 performance criteria for the chemical analysis of portland cements. From a letter by W.C. Hanna to the Working Committee on Methods of Chemical Analysis, June 12, 1946.

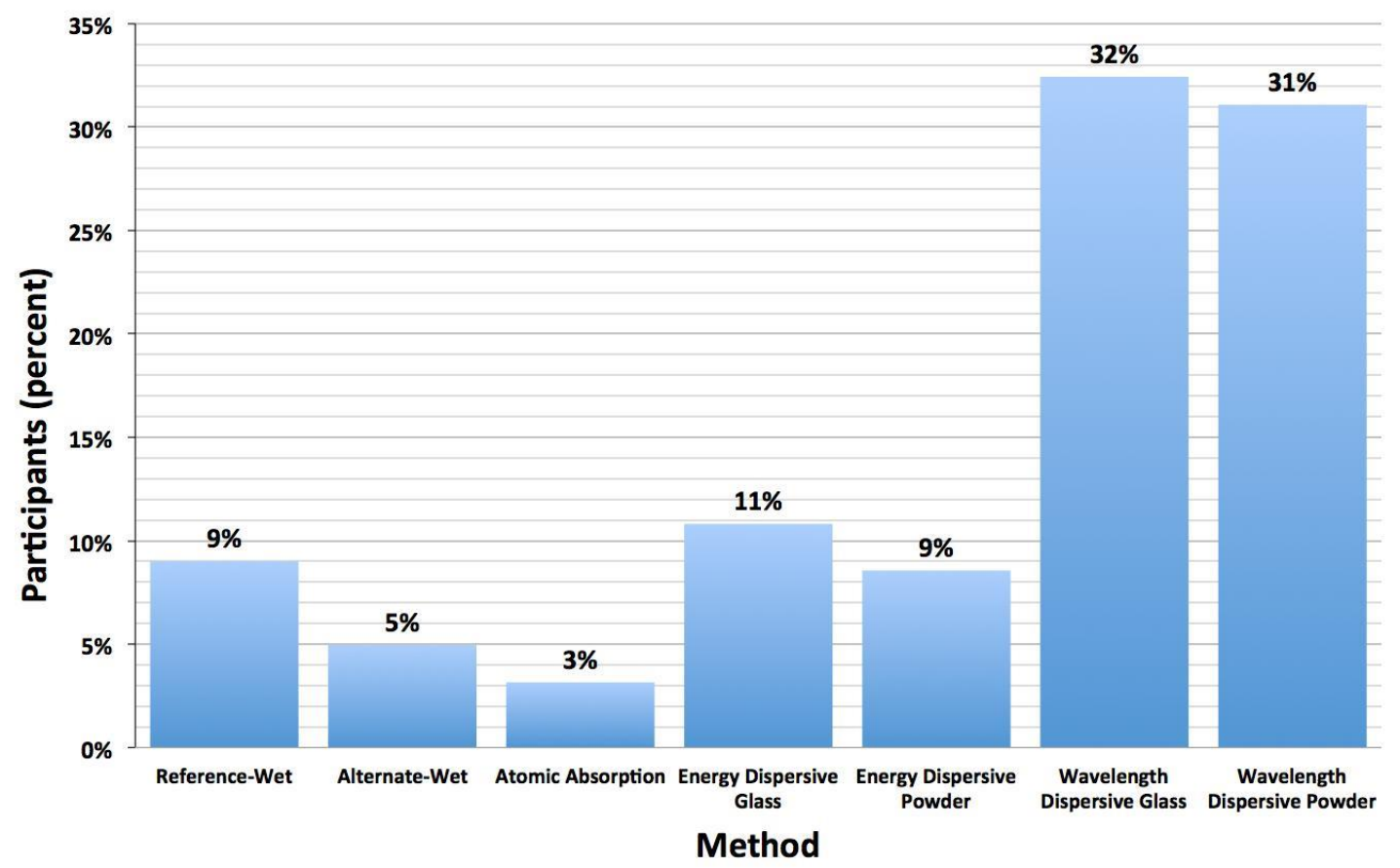

Figure 2 Reference and instrumental methods popularity from CCRL proficiency test data shows that over $80 \%$ of the labs use X-ray methods for bulk chemical analysis by either energy- or wavelength-dispersive analysis, using either powder or glass specimens. 


\section{Development of a Test Method for XRF Analysis of Hydraulic Cements}

Over $80 \%$ of the participants in the CCRL proficiency test program conduct XRF analysis using either a wavelength- or an energy-dispersive spectrometer [11]. The percentage of cement manufacturers that use XRF analysis is probably higher, and the remaining instrumental and traditional chemical methods of analysis are generally reserved as backup in case the principal XRF instrument is down for repair. In ASTM C114 terminology, the XRF method, atomic absorption and inductively coupled plasma spectrometry fall under rapid methods, reflecting the longer times required to perform the traditional chemical analyses. Current practice for chemical analysis follows ASTM C114, which states that any method of analysis may be used as long as it can be demonstrated to conform to precision and bias performance criteria of Table 1 in ASTM C114 [2]. This means that the rapid methods require qualification where results of six of seven certified reference materials must fall within the qualification criteria of Table 1; the seventh measurement must fall with twice the precision criteria in Table 1 . The qualification limits were originally published in 1946 and remain the same today. Bias criteria were originally based upon the maximum difference of three determinations, whereas today the criteria are a maximum limit on the difference of the mean of two determinations and an accepted reference value.

ASTM C 1.23 initiated an inter-laboratory study for XRF Analysis to establish a data set for assessing precision of the method being developed. This method does not provide a set of instructions to prepare specimens and perform an analysis, but rather outlines a goal of analysis of major and minor elements by XRF with use of either of two specimen preparation procedures: pressed powder and fused glass. Guidelines as a draft standard are provided for both the pressed pellet and the fused glass preparations.

For the inter-laboratory study, three pairs of cements were distributed to participants with approximately 45 laboratories participating. This report covers the first set of cements, which will be referred to as Cement A and Cement B, originating from the CCRL proficiency test program cements 163 and 164, respectively. Each laboratory was asked to follow their own standard operating procedure (SOP), as long as it fell within the draft standard guidelines, and prepare and analyze three specimens (replicates) to be analyzed twice each (duplicates) on two different days for a total of six specimens and twelve analyses (Appendix A). Subsequent studies will contain ASTM C595 1s cements (samples C D), portland cements with ca. $5 \%$ limestone addition (samples E F), and ASTM C595 1p cements with fly ash (samples $\mathrm{G} \mathrm{H}$ ). Results and data from each sample set will be reported separately.

Sample preparation for XRF can be achieved using either of two distinct methods: a pressed powder and a fused glass disk. Pressed powder specimens are typically ground in a tungsten carbide ring and puck mill with a binding agent to reduce the particle size and provide a packed powder mount that will remain intact for transport and analysis. The advantages of this preparation method include the simplicity and better detection limits while disadvantages include what is known as the "mineralogical effect", which requires a similar matrix between a bracketed calibration and unknown specimens for the calibrations to be valid. The potential for bias due to the mineralogical effect will be greater for the 
second, third, and fourth cement pairs, which will contain slag additions at the $50 \%$ level and limestone additions at a level of less than $5 \%$, and fly ash, respectively. The fused disk preparation eliminates the potentially adverse effects of discrete mineral phases by dissolving the cement in a flux and fusing the mixture into a homogeneous glass disk. However, the fluxing process is subject to volatization of some analytes if the heating process is not carefully controlled.

The data analysis follows ASTM E 691 [12]"Standard Practice for Conducting an Interlaboratory Study to Determine the Precision of a Test Method," and was performed using the Dataplot ${ }^{1}$ software. Data were compiled by the CCRL staff into a database and exported to a spreadsheet format for subsequent processing and analysis. The terms used in ASTM E691 and the means of their calculation are presented in Figure 3, with the last six terms being used subsequently in the evaluation and presentation of the results.

The layout of this report consists of some background for chemical analysis of cements, information on the interlaboratory study, methodology behind the precision calculations, a summary table for all reported elements, and individual analyte (as oxides) results in table and graphical form, along with comparisons to previous studies. The calculations provide precision estimates for consideration by the C01.23 Subcommittee on compositional analysis for use in developing a draft standard test method for XRF analysis of hydraulic cements. The draft method uses a qualification approach, similar to that used in ASTM $\mathrm{C} 114$, having criteria for precision as well as accuracy. Unfortunately, the process and data used to develop the C114 Table 1 criteria are no longer available, so while precision is calculated here, criteria for accuracy will require careful consideration by the subcommittee. If the predominant sources of uncertainty lie in the laboratory protocol - the sample preparation, the calibrations, and the analyses - the differences between laboratory results reflect the combined within-laboratory uncertainties and laboratory-specific bias based upon protocol. As was done with the XRD test method C1365, the proposed measure of accuracy uses the prediction interval to establish criteria for laboratory bias limits.

\footnotetext{
${ }^{1}$ http://www.itl.nist.gov/div898/software/dataplot/homepage.htm
} 
$n=$ number of test results per cell

p = number of laboratories

$x=$ individual test result

$\overline{\mathbf{x}}=$ cell average

$\bar{X}=$ average of cell averages for one material

$s=$ cell standard deviation

$\mathrm{d}=$ cell deviation

$\sum_{i=1}^{n} \bar{x} / n$

$\sum_{i=1}^{p} \bar{x} / p$

$\sum_{i=1}^{n}(x-\bar{x})^{2} /(n-1)$

$(\bar{x}-\bar{X})$

$S_{\overline{\mathbf{x}}}=$ standard deviation of cell averages

$\sum_{i=1}^{p}(d)^{2} /(p-1)$

$S_{r}=$ repeatability standard deviation

$\sqrt{\sum_{i=1}^{p} s^{2} / p}$

$s_{R}=$ reproducibility standard deviation

$\sqrt{s_{\bar{x}}^{2}+s_{r}^{2}(n-1) / n}$

$h=$ between-laboratory consistency

$d / s_{\bar{x}}$

$\mathbf{k}=$ within-laboratory consistency

$s / s_{r}$

$\mathbf{r}=95 \%$ repeatability statistic

$2.8 * s_{r}$

$\mathbf{R}=95 \%$ reproducibility statistic

$2.8 * s_{R}$

Figure 3 Calculated values for the determination of within and between lab precision 


\section{Measurement Precision}

Uncertainties in bulk oxide measurements originate from a number of sources: consistency and bias in specimen preparations, standardization, data collection procedures, and analysis protocol. Measurements are estimates of the actual value being measured and ideally have some statement of uncertainty. This may also be estimated through an interlaboratory study, which provides estimates on precision, or random error (Type A) and bias, or systematic error (Type B) uncertainty. ASTM defines precision as "the closeness of agreement between independent test results obtained under stipulated conditions (the standard test procedure), which may be expressed as a standard deviation (1- $\sigma)$." Precision is further differentiated by that achieved within a laboratory by a single instrument (and operator, or procedure), called repeatability, and that between different laboratories, called reproducibility as a single standard deviation or a 95 \% limit as defined by E691 and presented below:

\section{$\underline{\text { Repeatability: Precision under repeatability conditions }}$}

Repeatability limit (r): "The value below which the absolute difference between two individual test results obtained under repeatability conditions may be expected to occur with a probability of approximately $0.95(95 \%)$ "

The repeatability limit is $2.8\left(1.96^{*} \sqrt{2}\right)$ times the repeatability standard deviation

\section{$\underline{\text { Reproducibility: Precision under reproducibility conditions }}$}

Reproducibility limit (R): The value below which the absolute difference between two test results obtained under reproducibility conditions may be expected to occur with a probability of approximately $0.95(95 \%)$

The reproducibility limit is $2.8\left(1.96^{*} \sqrt{ } 2\right)$ times the reproducibility standard deviation

\section{Measurement Accuracy and Method Bias}

ASTM defines accuracy as "the closeness of agreement between a test result and a accepted reference value", which includes both random and systematic error. The qualification criteria in C 114, Table 1, Column 3 addresses accuracy. Bias is defined as "the difference between the expectation of the test results and an accepted reference value", and reflects the systematic error. A meaningful estimate of method bias is more difficult to extract from interlaboratory studies if an explicit protocol is not available, even if certified reference materials are available. Systematic error introduced by individual lab protocols dominates that of the method, making a universal bias correction difficult to estimate and apply. In addition, the cements used in this program were not reference materials (due to the number of participants), so a bias calculation is not possible. These materials were specially homogenized and packaged as part of the CCRL chemical proficiency test program. 


\section{Outlier Identification}

Participation in the inter-laboratory study (ILS) was open to all interested laboratories and was not restricted based on the laboratory staff's years of experience. In addition, the ILS did not specify an explicit method for preparation and analysis. These two factors contributed to the overall uncertainty in the measurement data. Some means of identification of outlying data was necessary to exclude any outlying results and their influences on the calculated precision and consensus values. A graphical representation of this process is provided in Figure 4 where the individual replicate data from powder data with results plotted with cement $A$ on the $\mathrm{x}$-axis and cement $\mathrm{B}$ on the $\mathrm{y}$-axis. The vertical and horizontal axes represent the consensus value means for cements $\mathrm{A}$ and $\mathrm{B}$, respectively for the original data (before outlier identification). Like a Youden plot, this plot of the data pairs provides some insight, based on the degree and directions of dispersion of the results. In the absence of bias, the point pattern is roughly circular, and the dispersion along a diagonal from the lower-left, to the upper-right quadrant represents systematic error, and dispersion that is orthogonal to this direction represents the random error (precision).

In the example for $\mathrm{CaO}$ by the powder preparation, there were three laboratories that reported data that were considered outliners. Lab 1 (labeled $\mathrm{O}$ 1) data, which was clustered along the diagonal well below the other values, exhibits significant systematic error for both cements, which may represent either a calibration or preparation error. Lab 3 (O 3 ) data, which was scattered in both along the diagonal and perpendicular to the diagonal, exhibit significant systematic and random errors. Finally, lab 20 (O 20) reported an errant value for one replicate of cement A. This might represent an error in the sample preparation or a data entry error. It was determined that the latter was most likely as the duplicate of this sample did not exhibit a similarly low value for cement A. In this case, no attempt was made to fix the data, and that analyte from that lab was removed for the final analysis to maintain the balanced data set required by E691.

The plots in Figure 5 of the laboratory means and standard deviation are also useful for rapid visualization of overall performance by laboratory and material. The means plots represent the mean of the three replicates for cements A and B (labeled as Material 1 and 2) against the consensus value. The mean values by laboratory have the effect of averaging out the random error in the analyses, providing a more robust estimate for each lab. Lab 1 stands out in producing consistently low values, which are those in the lower-left quadrant (O 1) of Figure 4. Precision problems of Lab 3 (cement B, material 2) and Lab 20 (cement A, material 1) are seen in the standard deviation (SD) plot.

Quantitative assessment of within- and between-lab precision is represented by the $h$ and $k$ statistics [12], which can take the table form or be expressed in a plot. The $h$ and $k$ consistency statistics, measures of the lab's within- and between-laboratory precision, are shown in Figure 6. These statistics were used to identify outlying lab data in the original data set, which were subsequently excluded on an analyte-by-analyte basis. The rationale for this being applied on a one time only basis was that it aided in the identification of unusual data due to standardization and procedural error, or errors on the reported values due to data entry. No attempt was made to evaluate the data and fix errant values due to 
entry error. ASTM E691 requires a balanced data set so if a lab submitted an incomplete data set or was flagged as an outlier due to an errant value, the entire data set for that analyte was eliminated.

\section{Included Appendices}

The analyses for each oxide, the letter of invitation to participate, and the raw data for each analyte are included in the appendices. Appendix A provides the analysis for each oxide, along with a comparison of the reported precision to the precision of other methods.

Appendix B provides the invitation to participate letter that provides instructions to the laboratory regarding the measurement and the reporting of values. Appendices $C$ and $D$ provides the raw data that provided by the laboratories for the glass and powder preparations, respectively, and Appendix E provides the raw data by analyte with index values for cement and replicate.

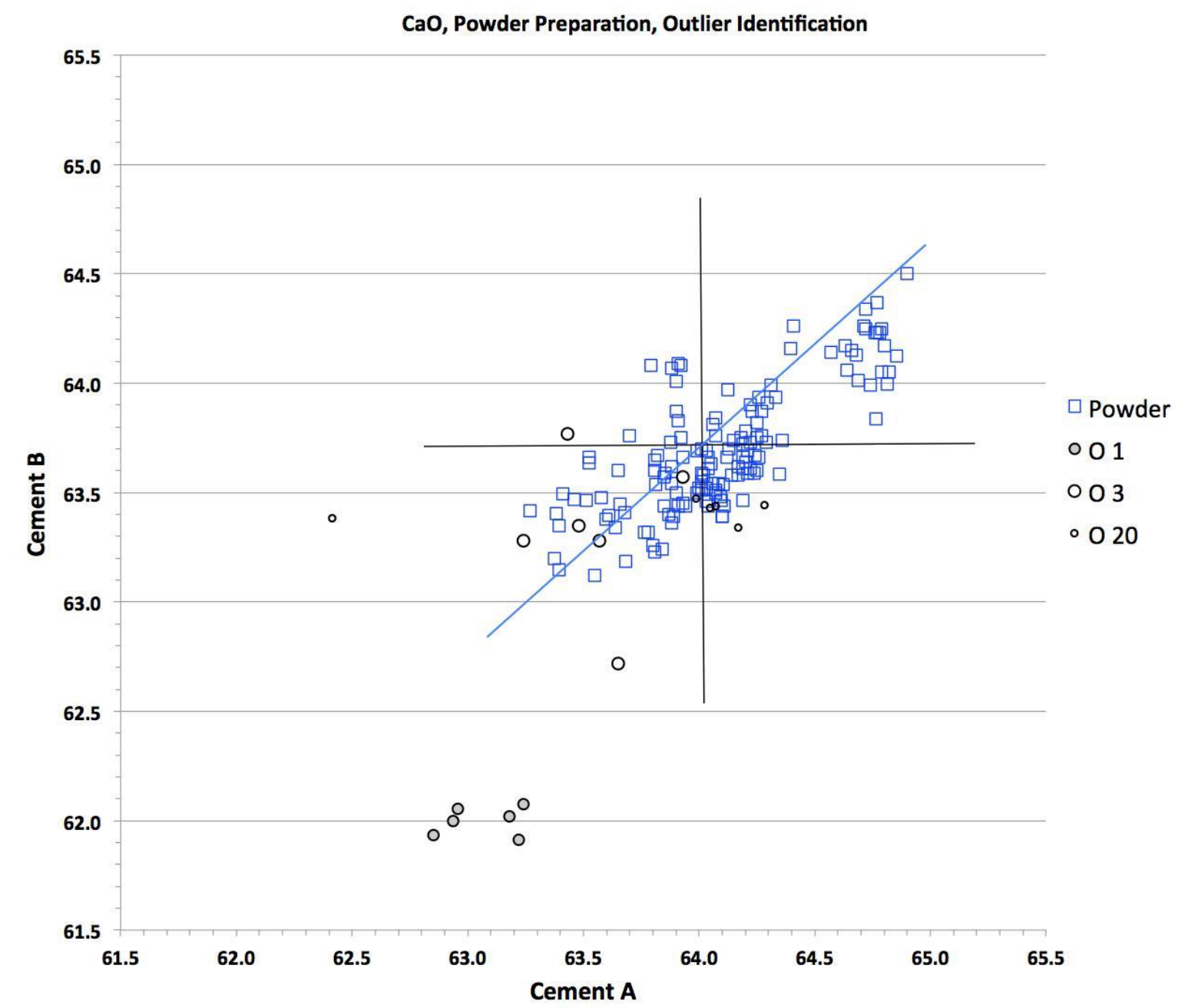

Figure 4 Scatter plot for $\mathrm{CaO}$, XRF-powder preparation as boxes with the "o" symbol noting excluded data due to exceeding the $\mathrm{h}$ or $\mathrm{k}$ statistics. 
CaO XRF, Powder Prep., Cements A B, Rep. 1
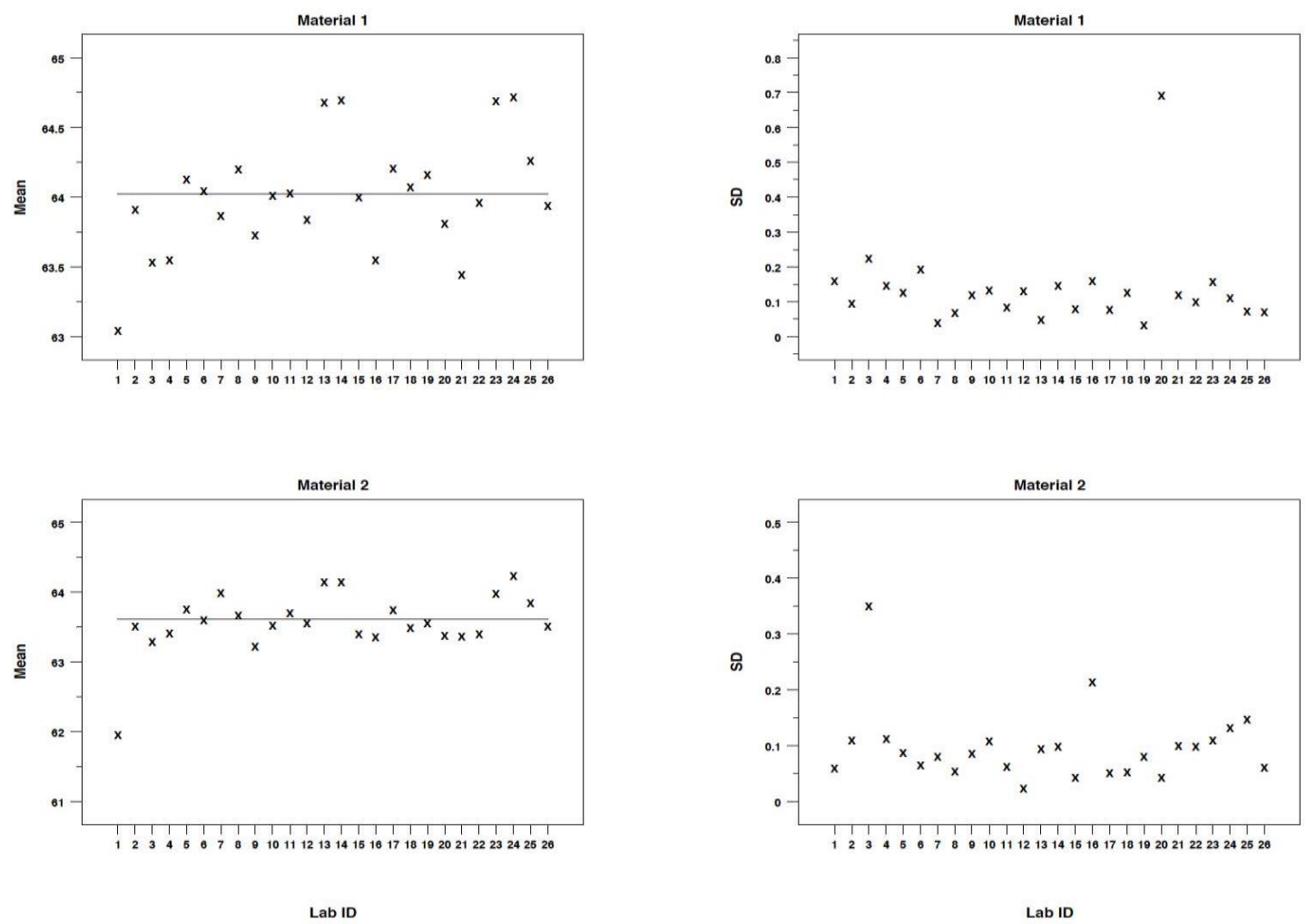

Figure 5 Lab means and standard deviations for $\mathrm{CaO}$, powder preparation for cement A (Material 1) and cement B (Material 2).

\section{Measurement Precision}

The results in Appendix A are condensed into Table 1, which presents the results by method, and includes the $1 \sigma$ within-laboratory $(S r)$, the $1 \sigma$ between-laboratory $(s R)$. The table also includes the appropriate ASTM d2s, the $95 \%$ limits on the difference between two test results, as $r$ and $R$, which represent a pooled standard deviation for the two replicates for both cements A and B. In addition, the results were pooled across glass and powder methods for consideration and are shown in the right-hand side of the table. More detailed summaries by method and cement are found in Appendix B for the glass preparation and Appendix $\mathrm{C}$ for the powder preparation. Appendix D contains the raw data used in this analysis. 

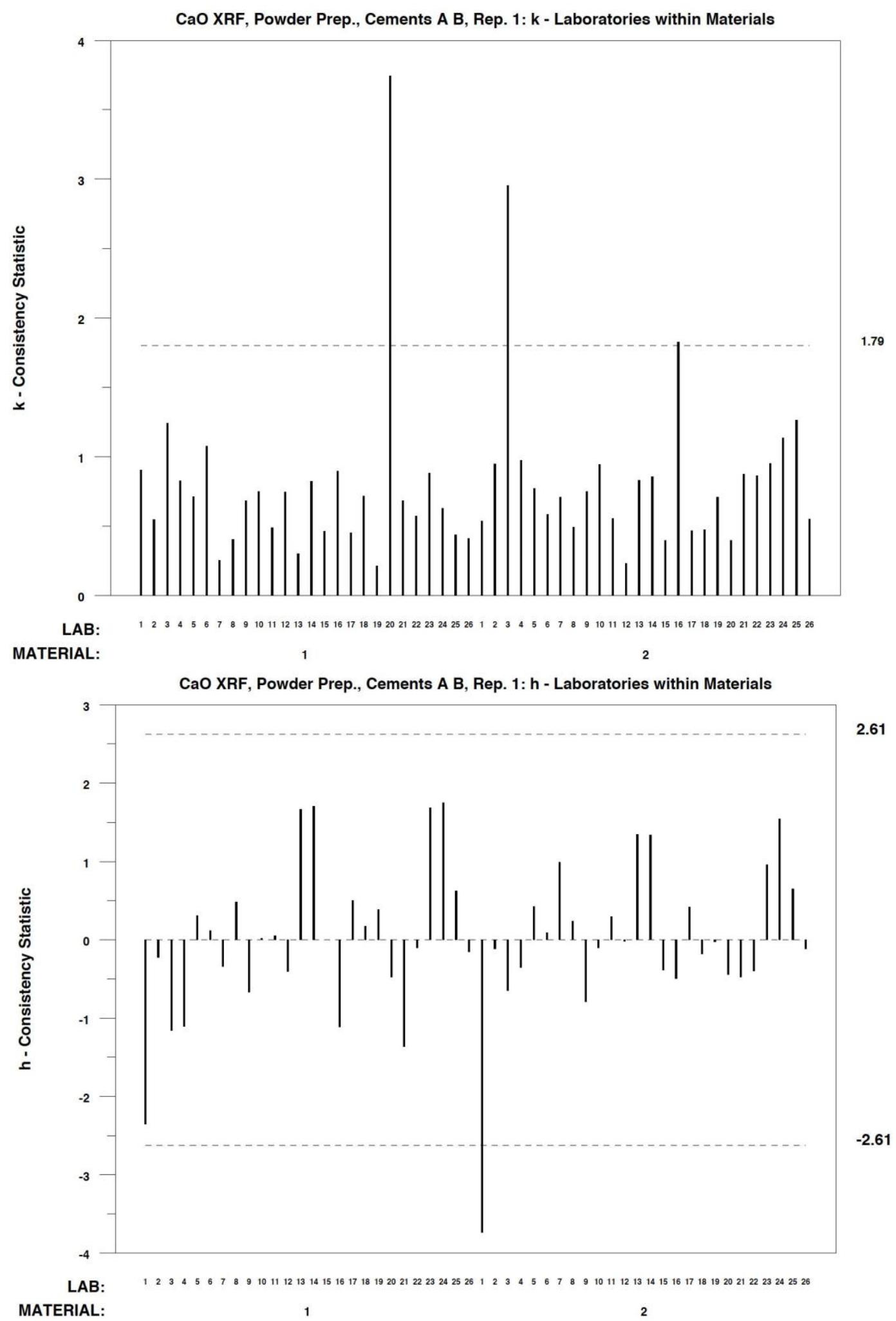

Figure $6 \mathrm{~h}$ and $\mathrm{k}$ statistic plots illustrate the within- $(k)$ and between-laboratory $(h)$ precision and are useful in identifying unusual results that may be considered outliers. The dashed line marks the limits for each statistic. 
Table 1 Pooled Results for XRF-Glass, XRF-Powder and Combined Powder and Glass Methods, when the results are expressed as a percent. The values include the $1 \sigma$ within-laboratory $(\mathrm{Sr})$, the $1 \sigma$ between-laboratory (sR), and the ASTM d2s values for the $95 \%$ limit on the difference between two test results (r) and (R).

\begin{tabular}{|c|c|c|c|c|c|c|c|c|c|c|c|c|}
\hline \multirow[b]{2}{*}{ Analyte } & \multicolumn{4}{|c|}{ Glass } & \multicolumn{4}{|c|}{ Powder } & \multicolumn{4}{|c|}{ Pooled Glass and Powder } \\
\hline & $S r$ & $s R$ & $\mathbf{r}$ & $\mathbf{R}$ & $\mathrm{Sr}$ & $s R$ & $\mathbf{r}$ & $\mathbf{R}$ & $S r$ & $s R$ & $\mathbf{r}$ & $\mathbf{R}$ \\
\hline $\mathrm{CaO}$ & 0.119 & 0.372 & 0.334 & 1.043 & 0.109 & 0.337 & 0.306 & 0.942 & 0.114 & 0.355 & 0.321 & 0.994 \\
\hline $\mathrm{SiO}_{2}$ & 0.054 & 0.106 & 0.151 & 0.298 & 0.056 & 0.161 & 0.155 & 0.450 & 0.055 & 0.136 & 0.153 & 0.381 \\
\hline $\mathrm{Al}_{2} \mathrm{O}_{3}$ & 0.021 & 0.057 & 0.058 & 0.160 & 0.022 & 0.096 & 0.063 & 0.266 & 0.022 & 0.079 & 0.060 & 0.220 \\
\hline $\mathrm{Fe}_{2} \mathrm{O}_{3}$ & 0.013 & 0.032 & 0.038 & 0.090 & 0.016 & 0.046 & 0.045 & 0.130 & 0.015 & 0.040 & 0.042 & 0.112 \\
\hline $\mathrm{SO}_{3}$ & 0.056 & 0.085 & 0.156 & 0.236 & 0.024 & 0.119 & 0.065 & 0.334 & 0.043 & 0.104 & 0.120 & 0.289 \\
\hline $\mathrm{MgO}$ & 0.012 & 0.026 & 0.034 & 0.077 & 0.012 & 0.065 & 0.033 & 0.185 & 0.012 & 0.050 & 0.033 & 0.142 \\
\hline $\mathrm{Na}_{2} \mathrm{O}$ & 0.023 & 0.029 & 0.062 & 0.080 & 0.005 & 0.019 & 0.013 & 0.055 & 0.016 & 0.025 & 0.045 & 0.069 \\
\hline $\mathrm{K}_{2} \mathrm{O}$ & 0.004 & 0.009 & 0.010 & 0.025 & 0.004 & 0.012 & 0.010 & 0.035 & 0.004 & 0.011 & 0.010 & 0.031 \\
\hline $\mathrm{TiO}_{2}$ & 0.004 & 0.008 & 0.010 & 0.020 & 0.003 & 0.007 & 0.010 & 0.020 & 0.004 & 0.008 & 0.010 & 0.020 \\
\hline $\mathrm{P}_{2} \mathrm{O}_{5}$ & 0.003 & 0.004 & 0.010 & 0.016 & 0.007 & 0.009 & 0.017 & 0.025 & 0.005 & 0.007 & 0.014 & 0.021 \\
\hline $\mathrm{Cl}$ (both) & 0.007 & 0.050 & 0.021 & 0.142 & 0.007 & 0.050 & 0.021 & 0.142 & 0.007 & 0.050 & 0.021 & 0.142 \\
\hline
\end{tabular}




\section{Prediction Intervals}

ASTM C114 qualification criteria specified in Table 1 includes an accuracy criterion for each analyte, by limiting the maximum difference between the mean of two duplicates and a certificate value from a certified reference material. A two-sided $95 \%$ prediction interval for the mean of two test results is presented in Table 2 for each analyte. This interval defines the maximum difference one might expect relative to a certified value of a reference material based upon the mean of two separate determinations [13]. This table provides the qualification criteria of column 3 of Table 1 in ASTM C114, titled "Maximum difference of the average of duplicates from CRM certificate values".

Table $295 \%$ Prediction Interval designed to bracket values of a mean of $k=2$ measurements. The mean result should differ from the known value of the certified reference material by no more than the value shown for each analyte.

\begin{tabular}{lccc}
\hline & \multicolumn{3}{c}{ Prediction Interval k=2 } \\
& Glass & Powder & Pooled \\
\hline $\mathbf{C a O}$ & 0.569 & 0.477 & 0.492 \\
$\mathbf{S i O}_{\mathbf{2}}$ & 0.222 & 0.367 & 0.287 \\
$\mathbf{A l}_{\mathbf{2}} \mathbf{O}_{\mathbf{3}}$ & 0.106 & 0.149 & 0.122 \\
$\mathbf{F e}_{\mathbf{2}} \mathbf{O}_{3}$ & 0.057 & 0.069 & 0.059 \\
$\mathbf{S O}_{\mathbf{3}}$ & 0.132 & 0.175 & 0.147 \\
$\mathbf{M g O}_{\mathbf{O}}$ & 0.037 & 0.085 & 0.063 \\
$\mathbf{N a}_{\mathbf{2}} \mathbf{O}$ & 0.038 & 0.031 & 0.032 \\
$\mathbf{K}_{\mathbf{2}} \mathbf{O}$ & 0.017 & 0.023 & 0.019 \\
$\mathbf{T i O}_{\mathbf{2}}$ & 0.013 & 0.011 & 0.011 \\
$\mathbf{P}_{\mathbf{2}} \mathbf{O}_{\mathbf{5}}$ & 0.007 & 0.017 & 0.012 \\
$\mathbf{C l}$ (both) & & & 0.064
\end{tabular}

\section{Summary}

Precision and accuracy estimates from analysis of data from an ASTM interlaboratory test program provide the basis for qualification statement for the new XRF standard test method now in development. The current qualification criteria are based upon traditional wet chemistry test methods, and the original data and means of their calculation of the qualification criteria data being no longer available. Following ASTM E691, precision values for within- and between-laboratory and their $95 \%$ limits have been determined. The accuracy criterion in ASTM C114, Table 1 is developed here using a two-point mean and $95 \%$ prediction interval. Together, these performance criteria will aid in facilitating accurate and consistent analyses of the bulk chemical compositions of hydraulic cements. 


\section{Appendix A. Individual Oxide Analysis}

The results by analyte and method are represented by a consistent set of plots and tables to illustrate the results, and by a summary table to provide a comparison to previous studies on precision. These results include:

- A box plot for Cement A and Cement B, which includes Reference Methods data from the CCRL proficiency test program,

- Summary tables of means, standard deviations, cell deviation, and $\mathrm{h}$ and $\mathrm{k}$ statistics (see Fig. 3),

- Summary tables by method and replicate for within- and between-laboratory precision expressed as a standard deviation and ASTM d2s $95 \%$ limits, and

- A bar chart comparing between lab standard deviation (reproducibility) to that of previous studies.

Each analyte, expressed as an oxide, is represented by box plots for both cements A and B that include the reference data from the CCRL proficiency test program for these cements for comparison, a table of results for each duplicate and replicate by sample preparation (glass and powder), a summary table for the precision calculations by cement, duplicate and replicate, and a bar chart illustrating the pooled results against the ASTM C114 criteria and reproducibility values calculated in other studies on analytical uncertainty.

Box plots are a graphical one-way ANOVA, enabling comparison of the two XRF preparation and the reference methods results through assessment of the alignment or misalignment of median values, differences in interquartile ranges, and the extant of the data extremes. The box plots presented represent the XRF data after outliers from the initial analysis have been removed.

Important features of the box plot are:

- the width of each box is proportional to sample size,

- the median value, identified by the $\mathrm{X}$ within the box, is used for its resistance to outliers,

- the interquartile range ("middle half") of the data are represented by the body of the box,

- the top and the bottom of the box represent the estimated $75 \%$ and $25 \%$ point, respectively, and

- the extremes (minimum and maximum) are represented by the ends of the straight lines projecting from the box. 
$\mathrm{SiO}_{2}$

SiO2
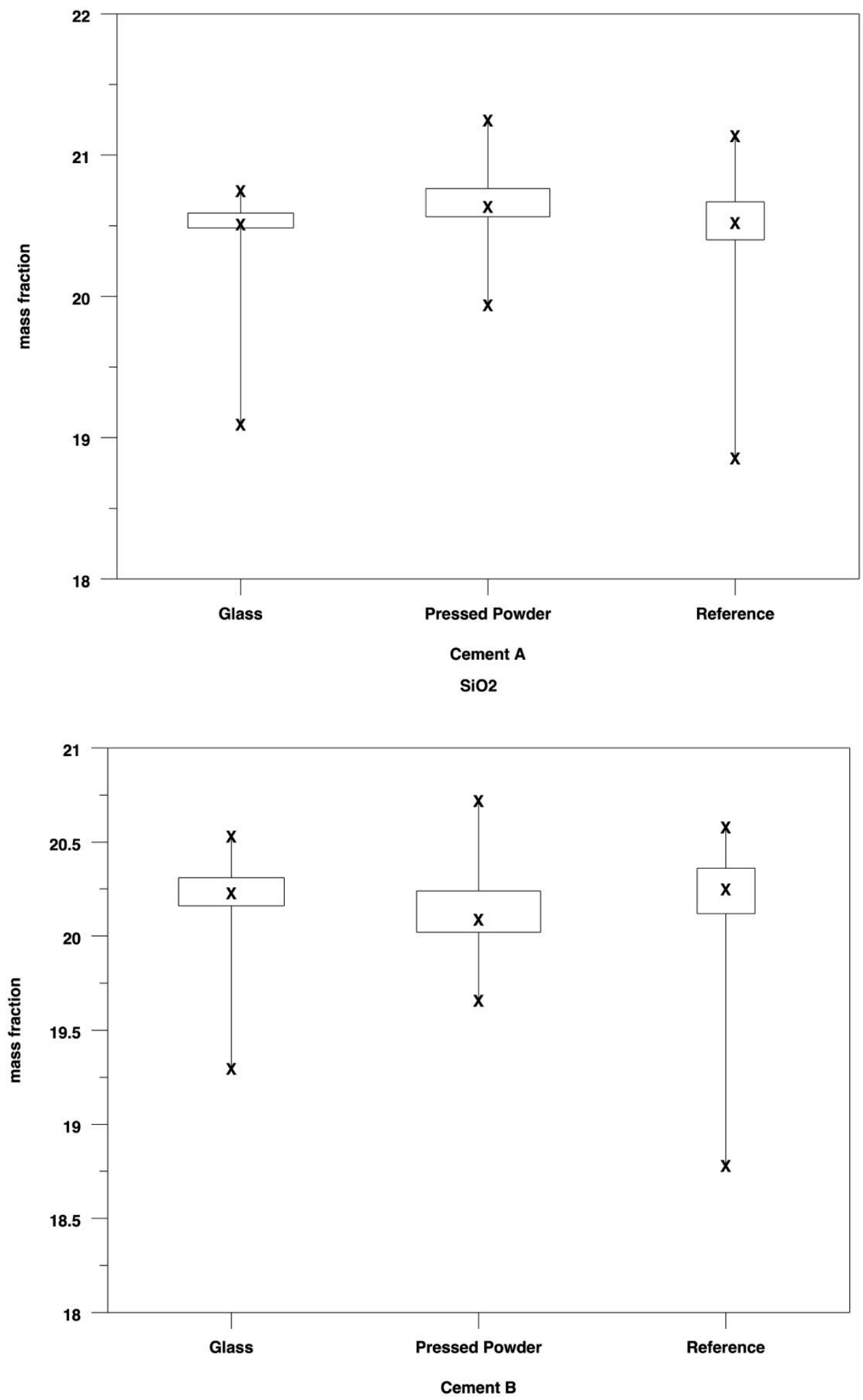

Figure $7 \mathrm{Box}$ plots for $\mathrm{SiO}_{2}$ for XRF glass and powder, and reference methods. 
$\mathrm{SiO}_{2}$ XRF, Cements A B, Glass, Replicate 1, Material: 1

\begin{tabular}{|c|c|c|c|c|c|}
\hline $\begin{array}{l}\text { Laboratory } \\
\text { Number }\end{array}$ & $\begin{array}{r}\text { Cell } \\
\text { Mean } \\
\end{array}$ & $\begin{array}{r}\text { Cell } \\
\text { SD } \\
\end{array}$ & $d$ & $\boldsymbol{h}$ & $\boldsymbol{k}$ \\
\hline 1 & 20.3965 & 0.0797 & -0.1385 & -1.85 & 1.62 \\
\hline 2 & 20.3835 & 0.0794 & -0.1515 & -2.02 & 1.61 \\
\hline 3 & 20.5300 & 0.0185 & -0.0050 & -0.07 & 0.37 \\
\hline 4 & 20.5267 & 0.0163 & -0.0084 & -0.11 & 0.33 \\
\hline 5 & 20.6005 & 0.0401 & 0.0655 & 0.87 & 0.81 \\
\hline 6 & 20.5283 & 0.0366 & -0.0067 & -0.09 & 0.74 \\
\hline 7 & 20.6633 & 0.0728 & 0.1283 & 1.71 & 1.48 \\
\hline 8 & 20.5020 & 0.0304 & -0.0330 & -0.44 & 0.62 \\
\hline 9 & 20.5167 & 0.0308 & -0.0184 & -0.24 & 0.62 \\
\hline 10 & 20.5383 & 0.0598 & 0.0033 & 0.04 & 1.21 \\
\hline 11 & 20.4650 & 0.0451 & -0.0700 & -0.93 & 0.91 \\
\hline 12 & 20.5817 & 0.0331 & 0.0466 & 0.62 & 0.67 \\
\hline 13 & 20.5660 & 0.0659 & 0.0310 & 0.41 & 1.34 \\
\hline 14 & 20.5233 & 0.0378 & -0.0117 & -0.16 & 0.77 \\
\hline 15 & 20.5450 & 0.0742 & 0.0100 & 0.13 & 1.50 \\
\hline 16 & 20.6633 & 0.0294 & 0.1283 & 1.71 & 0.60 \\
\hline 17 & 20.4952 & 0.0303 & -0.0399 & -0.53 & 0.61 \\
\hline 18 & 20.6050 & 0.0259 & 0.0700 & 0.93 & 0.52 \\
\hline \multicolumn{2}{|l|}{ Average of } & \multicolumn{4}{|c|}{$=20.53502$} \\
\hline Standard Devi & of cell averages & \multicolumn{4}{|c|}{$=0.07503$} \\
\hline Repeatability & rd Deviation & \multicolumn{4}{|c|}{0.04934} \\
\hline \multirow{2}{*}{\multicolumn{2}{|c|}{ Critical values $\mathrm{h}, \mathrm{k}$}} & \multicolumn{4}{|c|}{0.08751} \\
\hline & & \multicolumn{4}{|c|}{$2.53,1.78$} \\
\hline
\end{tabular}


$\mathrm{SiO}_{2}$ XRF, Cements A B, Glass, Replicate 1, Material: 2

\begin{tabular}{cclrrr}
\hline $\begin{array}{c}\text { Laboratory } \\
\text { Number }\end{array}$ & $\begin{array}{r}\text { Cell } \\
\text { Mean }\end{array}$ & $\begin{array}{r}\text { Cell } \\
\text { SD }\end{array}$ & $\boldsymbol{d}$ & $\boldsymbol{h}$ & $\boldsymbol{k}$ \\
\hline 1 & 20.2365 & 0.0805 & -0.0024 & -0.02 & 1.40 \\
2 & 20.2210 & 0.0873 & -0.0179 & -0.16 & 1.52 \\
3 & 20.1612 & 0.0169 & -0.0777 & -0.71 & 0.29 \\
4 & 20.2950 & 0.0327 & 0.0561 & 0.51 & 0.57 \\
5 & 20.3387 & 0.0279 & 0.0998 & 0.91 & 0.49 \\
6 & 20.2983 & 0.0744 & 0.0594 & 0.54 & 1.30 \\
7 & 20.3517 & 0.0809 & 0.1128 & 1.03 & 1.41 \\
8 & 20.2447 & 0.0229 & 0.0058 & 0.05 & 0.40 \\
9 & 20.1883 & 0.0194 & -0.0506 & -0.46 & 0.34 \\
10 & 20.1950 & 0.0497 & -0.0439 & -0.40 & 0.87 \\
11 & 19.9533 & 0.0622 & -0.2856 & -2.60 & 1.08 \\
12 & 20.2817 & 0.0279 & 0.0428 & 0.39 & 0.49 \\
13 & 20.1127 & 0.0735 & -0.1262 & -1.15 & 1.28 \\
14 & 20.2767 & 0.0367 & 0.0378 & 0.34 & 0.64 \\
15 & 20.2667 & 0.1141 & 0.0278 & 0.25 & 1.99 \\
16 & 20.4517 & 0.0492 & 0.2128 & 1.93 & 0.86 \\
17 & 20.1205 & 0.0197 & -0.1184 & -1.08 & 0.34 \\
18 & 20.3067 & 0.0216 & 0.0678 & 0.62 & 0.38 \\
\hline Average of cell averages & $=$ & 20.23890 & & \\
Standard Deviation of cell averages & $=$ & 0.10998 & & \\
Repeatability Standard Deviation & $=$ & 0.05744 & & \\
Reproducibility Standard Deviation & $=$ & 0.12184 & & \\
Critical values h, k & & $=$ & $2.53,1.78$ & &
\end{tabular}


$\mathrm{SiO}_{2}$ XRF, Cements A B, Glass, Replicate 2, Material: 1

\begin{tabular}{|c|c|c|c|c|c|}
\hline $\begin{array}{c}\text { Laboratory } \\
\text { Number }\end{array}$ & $\begin{array}{r}\text { Cell } \\
\text { Mean } \\
\end{array}$ & $\begin{array}{r}\text { Cell } \\
\text { SD }\end{array}$ & $d$ & $\boldsymbol{h}$ & $\boldsymbol{k}$ \\
\hline 1 & 20.3812 & 0.0655 & -0.1504 & -1.84 & 1.40 \\
\hline 2 & 20.3577 & 0.0782 & -0.1739 & -2.13 & 1.67 \\
\hline 3 & 20.5403 & 0.0151 & 0.0087 & 0.11 & 0.32 \\
\hline 4 & 20.5233 & 0.0234 & -0.0083 & -0.10 & 0.50 \\
\hline 5 & 20.6037 & 0.0416 & 0.0721 & 0.88 & 0.89 \\
\hline 6 & 20.5183 & 0.0640 & -0.0133 & -0.16 & 1.37 \\
\hline 7 & 20.6567 & 0.0659 & 0.1251 & 1.53 & 1.41 \\
\hline 8 & 20.4963 & 0.0483 & -0.0353 & -0.43 & 1.03 \\
\hline 9 & 20.4833 & 0.0308 & -0.0483 & -0.59 & 0.66 \\
\hline 10 & 20.5133 & 0.0383 & -0.0183 & -0.22 & 0.82 \\
\hline 11 & 20.4800 & 0.0316 & -0.0516 & -0.63 & 0.68 \\
\hline 12 & 20.5567 & 0.0468 & 0.0251 & 0.31 & 1.00 \\
\hline 13 & 20.6117 & 0.0486 & 0.0801 & 0.98 & 1.04 \\
\hline 14 & 20.5033 & 0.0314 & -0.0283 & -0.35 & 0.67 \\
\hline 15 & 20.5617 & 0.0605 & 0.0301 & 0.37 & 1.30 \\
\hline 16 & 20.6533 & 0.0333 & 0.1217 & 1.49 & 0.71 \\
\hline 17 & 20.5030 & 0.0246 & -0.0286 & -0.35 & 0.53 \\
\hline 18 & 20.6250 & 0.0351 & 0.0934 & 1.14 & 0.75 \\
\hline \multicolumn{2}{|c|}{ Average of cell averages } & \multicolumn{2}{|c|}{$=\quad 20.53160$} & & \\
\hline Standard Devi & of cell averages & \multicolumn{2}{|c|}{0.08179} & & \\
\hline Repeatability & rd Deviation & \multicolumn{2}{|c|}{0.04668} & & \\
\hline Reproducibili & dard Deviation & \multicolumn{2}{|c|}{0.09222} & & \\
\hline \multicolumn{2}{|c|}{ Critical values $\mathrm{h}, \mathrm{k}$} & $=$ & $2.53,1.78$ & & \\
\hline
\end{tabular}


$\mathrm{SiO}_{2}$ XRF, Cements A B, Glass, Replicate 2, Material: 2

\begin{tabular}{|c|c|c|c|c|c|}
\hline $\begin{array}{l}\text { Laboratory } \\
\text { Number }\end{array}$ & $\begin{array}{r}\text { Cell } \\
\text { Mean } \\
\end{array}$ & $\begin{array}{r}\text { Cell } \\
\text { SD } \\
\end{array}$ & $d$ & $\boldsymbol{h}$ & $\boldsymbol{k}$ \\
\hline 1 & 20.1507 & 0.0755 & -0.0829 & -0.81 & 1.24 \\
\hline 2 & 20.1672 & 0.1104 & -0.0664 & -0.65 & 1.82 \\
\hline 3 & 20.1625 & 0.0236 & -0.0711 & -0.69 & 0.39 \\
\hline 4 & 20.2950 & 0.0302 & 0.0614 & 0.60 & 0.50 \\
\hline 5 & 20.3352 & 0.0500 & 0.1016 & 0.99 & 0.82 \\
\hline 6 & 20.2683 & 0.0783 & 0.0348 & 0.34 & 1.29 \\
\hline 7 & 20.3617 & 0.0781 & 0.1281 & 1.25 & 1.29 \\
\hline 8 & 20.2377 & 0.0227 & 0.0041 & 0.04 & 0.37 \\
\hline 9 & 20.2217 & 0.0512 & -0.0119 & -0.12 & 0.84 \\
\hline 10 & 20.1983 & 0.0700 & -0.0352 & -0.34 & 1.15 \\
\hline 11 & 19.9800 & 0.0790 & -0.2536 & -2.47 & 1.30 \\
\hline 12 & 20.2233 & 0.0273 & -0.0102 & -0.10 & 0.45 \\
\hline 13 & 20.1775 & 0.0370 & -0.0561 & -0.55 & 0.61 \\
\hline 14 & 20.2867 & 0.0266 & 0.0531 & 0.52 & 0.44 \\
\hline 15 & 20.2583 & 0.1134 & 0.0248 & 0.24 & 1.87 \\
\hline 16 & 20.4400 & 0.0297 & 0.2064 & 2.01 & 0.49 \\
\hline 17 & 20.1300 & 0.0160 & -0.1036 & -1.01 & 0.26 \\
\hline 18 & 20.3100 & 0.0352 & 0.0764 & 0.74 & 0.58 \\
\hline \multicolumn{2}{|c|}{ Average of cell averages } & \multicolumn{4}{|c|}{$=20.23355$} \\
\hline Standard Devi & of cell averages & \multicolumn{4}{|c|}{$=\quad 0.10279$} \\
\hline \multicolumn{2}{|c|}{ Repeatability Standard Deviation } & \multicolumn{4}{|c|}{0.06074} \\
\hline \multicolumn{2}{|c|}{ Reproducibility Standard Deviation } & \multicolumn{4}{|c|}{0.11679} \\
\hline \multicolumn{2}{|c|}{ Critical values $\mathrm{h}, \mathrm{k}$} & \multicolumn{4}{|c|}{$2.53,1.78$} \\
\hline
\end{tabular}


$\mathrm{SiO}_{2}$ XRF, Cements A B, Powder, Replicate 1, Material: 1

\begin{tabular}{|c|c|c|c|c|c|}
\hline $\begin{array}{c}\text { Laboratory } \\
\text { Number }\end{array}$ & $\begin{array}{r}\text { Cell } \\
\text { Mean }\end{array}$ & $\begin{array}{r}\text { Cell } \\
\text { SD }\end{array}$ & $d$ & $\boldsymbol{h}$ & $\boldsymbol{k}$ \\
\hline 1 & 20.6900 & 0.0874 & 0.0200 & 0.16 & 1.56 \\
\hline 2 & 20.6093 & 0.0540 & -0.0606 & -0.48 & 0.96 \\
\hline 3 & 20.6337 & 0.0546 & -0.0363 & -0.29 & 0.97 \\
\hline 4 & 20.6283 & 0.0500 & -0.0416 & -0.33 & 0.89 \\
\hline 5 & 20.6617 & 0.0705 & -0.0083 & -0.07 & 1.26 \\
\hline 6 & 20.9333 & 0.0197 & 0.2634 & 2.09 & 0.35 \\
\hline 7 & 20.7717 & 0.0382 & 0.1017 & 0.81 & 0.68 \\
\hline 8 & 20.7500 & 0.0219 & 0.0800 & 0.64 & 0.39 \\
\hline 9 & 20.4383 & 0.0407 & -0.2316 & -1.84 & 0.73 \\
\hline 10 & 20.7512 & 0.0294 & 0.0812 & 0.65 & 0.52 \\
\hline 11 & 20.5513 & 0.0341 & -0.1186 & -0.94 & 0.61 \\
\hline 12 & 20.6817 & 0.0838 & 0.0117 & 0.09 & 1.49 \\
\hline 13 & 20.5812 & 0.0326 & -0.0888 & -0.71 & 0.58 \\
\hline 14 & 20.6105 & 0.0690 & -0.0595 & -0.47 & 1.23 \\
\hline 15 & 20.6467 & 0.0592 & -0.0233 & -0.19 & 1.06 \\
\hline 16 & 20.4917 & 0.0299 & -0.1783 & -1.42 & 0.53 \\
\hline 17 & 20.5500 & 0.0805 & -0.1200 & -0.95 & 1.44 \\
\hline 18 & 20.5432 & 0.0553 & -0.1268 & -1.01 & 0.99 \\
\hline 19 & 20.8183 & 0.0725 & 0.1484 & 1.18 & 1.29 \\
\hline 20 & 20.7950 & 0.0812 & 0.1250 & 0.99 & 1.45 \\
\hline 21 & 20.7055 & 0.0404 & 0.0355 & 0.28 & 0.72 \\
\hline 22 & 20.8967 & 0.0432 & 0.2267 & 1.80 & 0.77 \\
\hline
\end{tabular}

Average of cell averages $\quad=20.66996$

Standard Deviation of cell averages $=0.12579$

Repeatability Standard Deviation $=0.05606$

Reproducibility Standard Deviation $=0.13580$

Critical values $\mathrm{h}, \mathrm{k}=2.58,1.78$ 
$\mathrm{SiO}_{2}$ XRF, Cements A B, Powder, Replicate 1, Material: 2

\begin{tabular}{|c|c|c|c|c|c|}
\hline $\begin{array}{c}\text { Laboratory } \\
\text { Number }\end{array}$ & $\begin{array}{r}\text { Cell } \\
\text { Mean }\end{array}$ & $\begin{array}{r}\text { Cell } \\
\text { SD }\end{array}$ & $d$ & $\boldsymbol{h}$ & $k$ \\
\hline 1 & 20.1433 & 0.0339 & 0.0263 & 0.15 & $\overline{0.63}$ \\
\hline 2 & 19.7277 & 0.0464 & -0.3894 & -2.21 & 0.86 \\
\hline 3 & 20.0672 & 0.0822 & -0.0499 & -0.28 & 1.53 \\
\hline 4 & 20.2650 & 0.0288 & 0.1480 & 0.84 & 0.54 \\
\hline 5 & 20.3067 & 0.0463 & 0.1896 & 1.08 & 0.86 \\
\hline 6 & 20.3750 & 0.0547 & 0.2580 & 1.47 & 1.02 \\
\hline 7 & 20.5217 & 0.0417 & 0.4046 & 2.30 & 0.77 \\
\hline 8 & 20.0450 & 0.0592 & -0.0720 & -0.41 & 1.10 \\
\hline 9 & 19.8967 & 0.0413 & -0.2204 & -1.25 & 0.77 \\
\hline 10 & 20.4022 & 0.0481 & 0.2851 & 1.62 & 0.89 \\
\hline 11 & 19.9907 & 0.0290 & -0.1264 & -0.72 & 0.54 \\
\hline 12 & 20.0833 & 0.0771 & -0.0337 & -0.19 & 1.43 \\
\hline 13 & 20.0695 & 0.0285 & -0.0475 & -0.27 & 0.53 \\
\hline 14 & 19.9770 & 0.0715 & -0.1400 & -0.80 & 1.33 \\
\hline 15 & 20.0850 & 0.0451 & -0.0320 & -0.18 & 0.84 \\
\hline 16 & 20.0917 & 0.0445 & -0.0254 & -0.14 & 0.83 \\
\hline 17 & 19.9800 & 0.0860 & -0.1370 & -0.78 & 1.60 \\
\hline 18 & 20.0770 & 0.0656 & -0.0400 & -0.23 & 1.22 \\
\hline 19 & 20.1617 & 0.0436 & 0.0446 & 0.25 & 0.81 \\
\hline 20 & 20.0467 & 0.0520 & -0.0704 & -0.40 & 0.97 \\
\hline 21 & 20.0870 & 0.0594 & -0.0300 & -0.17 & 1.10 \\
\hline 22 & 20.1750 & 0.0423 & 0.0580 & 0.33 & 0.79 \\
\hline
\end{tabular}

Average of cell averages $\quad=\quad 20.11704$

Standard Deviation of cell averages $=0.17596$

Repeatability Standard Deviation $=0.05377$

Reproducibility Standard Deviation $=0.18268$

Critical values $\mathrm{h}, \mathrm{k}=2.58,1.78$ 
$\mathrm{SiO}_{2}$ XRF, Cements A B, Powder, Replicate 2, Material: 1

\begin{tabular}{|c|c|c|c|c|c|}
\hline $\begin{array}{c}\text { Laboratory } \\
\text { Number }\end{array}$ & $\begin{array}{r}\text { Cell } \\
\text { Mean }\end{array}$ & $\begin{array}{r}\text { Cell } \\
\text { SD }\end{array}$ & $d$ & $\boldsymbol{h}$ & $\boldsymbol{k}$ \\
\hline 1 & 20.7250 & 0.0628 & 0.0474 & 0.38 & $\overline{1.08}$ \\
\hline 2 & 20.5675 & 0.1028 & -0.1101 & -0.88 & 1.76 \\
\hline 3 & 20.6398 & 0.0484 & -0.0378 & -0.30 & 0.83 \\
\hline 4 & 20.6283 & 0.0611 & -0.0493 & -0.39 & 1.05 \\
\hline 5 & 20.6767 & 0.0812 & -0.0010 & -0.01 & 1.39 \\
\hline 6 & 20.9267 & 0.0137 & 0.2490 & 1.98 & 0.23 \\
\hline 7 & 20.7900 & 0.0335 & 0.1124 & 0.90 & 0.57 \\
\hline 8 & 20.7450 & 0.0187 & 0.0674 & 0.54 & 0.32 \\
\hline 9 & 20.4617 & 0.0392 & -0.2160 & -1.72 & 0.67 \\
\hline 10 & 20.7458 & 0.0279 & 0.0682 & 0.54 & 0.48 \\
\hline 11 & 20.5480 & 0.0412 & -0.1296 & -1.03 & 0.71 \\
\hline 12 & 20.6950 & 0.0896 & 0.0174 & 0.14 & 1.54 \\
\hline 13 & 20.6213 & 0.0259 & -0.0563 & -0.45 & 0.44 \\
\hline 14 & 20.6280 & 0.0709 & -0.0496 & -0.40 & 1.21 \\
\hline 15 & 20.6500 & 0.0740 & -0.0276 & -0.22 & 1.27 \\
\hline 16 & 20.5200 & 0.0363 & -0.1576 & -1.26 & 0.62 \\
\hline 17 & 20.5350 & 0.0771 & -0.1426 & -1.14 & 1.32 \\
\hline 18 & 20.5572 & 0.0614 & -0.1205 & -0.96 & 1.05 \\
\hline 19 & 20.8467 & 0.0717 & 0.1690 & 1.35 & 1.23 \\
\hline 20 & 20.8050 & 0.0493 & 0.1274 & 1.01 & 0.84 \\
\hline 21 & 20.6850 & 0.0492 & 0.0074 & 0.06 & 0.84 \\
\hline 22 & 20.9100 & 0.0424 & 0.2324 & 1.85 & 0.73 \\
\hline
\end{tabular}

Average of cell averages $\quad=20.67762$

Standard Deviation of cell averages $=0.12550$

Repeatability Standard Deviation $=0.05835$

Reproducibility Standard Deviation $=0.13634$

Critical values $\mathrm{h}, \mathrm{k} \quad=\quad 2.58,1.79$ 
$\mathrm{SiO}_{2}$ XRF, Cements A B, Powder, Replicate 2, Material: 2

\begin{tabular}{crrrrr}
\hline $\begin{array}{c}\text { Laboratory } \\
\text { Number }\end{array}$ & $\begin{array}{r}\text { Cell } \\
\text { Mean }\end{array}$ & $\begin{array}{r}\text { Cell } \\
\text { SD }\end{array}$ & $\boldsymbol{d}$ & $\boldsymbol{h}$ & $\boldsymbol{k}$ \\
\hline 1 & 20.1467 & 0.0216 & 0.0248 & 0.14 & 0.40 \\
2 & 19.7477 & 0.0374 & -0.3742 & -2.13 & 0.69 \\
3 & 20.0790 & 0.0811 & -0.0428 & -0.24 & 1.50 \\
4 & 20.2533 & 0.0320 & 0.1315 & 0.75 & 0.59 \\
5 & 20.3017 & 0.0286 & 0.1798 & 1.02 & 0.53 \\
6 & 20.3717 & 0.0578 & 0.2498 & 1.42 & 1.07 \\
7 & 20.5383 & 0.0343 & 0.4165 & 2.37 & 0.63 \\
8 & 20.0300 & 0.0498 & -0.0918 & -0.52 & 0.92 \\
9 & 19.9117 & 0.0431 & -0.2102 & -1.20 & 0.80 \\
10 & 20.4063 & 0.0496 & 0.2845 & 1.62 & 0.92 \\
11 & 19.9875 & 0.0357 & -0.1343 & -0.77 & 0.66 \\
12 & 20.0983 & 0.0833 & -0.0235 & -0.13 & 1.54 \\
13 & 20.1020 & 0.0293 & -0.0198 & -0.11 & 0.54 \\
14 & 19.9942 & 0.0684 & -0.1277 & -0.73 & 1.26 \\
15 & 20.0333 & 0.0561 & -0.0885 & -0.50 & 1.04 \\
16 & 20.0983 & 0.0475 & -0.0235 & -0.13 & 0.88 \\
17 & 19.9717 & 0.0842 & -0.1502 & -0.86 & 1.56 \\
18 & 20.0747 & 0.0752 & -0.0472 & -0.27 & 1.39 \\
19 & 20.2017 & 0.0392 & 0.0798 & 0.45 & 0.72 \\
20 & 20.0517 & 0.0591 & -0.0702 & -0.40 & 1.09 \\
21 & 20.0875 & 0.0677 & -0.0343 & -0.20 & 1.25 \\
22 & 20.1933 & 0.0372 & 0.0715 & 0.41 & 0.69 \\
\hline
\end{tabular}

Average of cell averages $\quad=\quad 20.12184$

Standard Deviation of cell averages $=0.17560$

Repeatability Standard Deviation $=0.05416$

Reproducibility Standard Deviation $=0.18243$

Critical values $\mathrm{h}, \mathrm{k}=2.58,1.79$ 
Table 3 Precision Statistics $\mathrm{SiO}_{2}$ XRF, Cements A B

XRF Glass, Replicate 1

\begin{tabular}{crrrrrr}
\hline Material & Xbar & $\boldsymbol{s}_{\boldsymbol{x}}$ & $\boldsymbol{s}_{\boldsymbol{r}}$ & $\boldsymbol{s}_{\boldsymbol{R}}$ & $\boldsymbol{r}$ & $\boldsymbol{R}$ \\
\hline 1 & 20.5350 & 0.0750 & 0.0493 & 0.0875 & 0.14 & 0.25 \\
2 & 20.2389 & 0.1100 & 0.0574 & 0.1218 & 0.16 & 0.34 \\
\hline
\end{tabular}

XRF Glass, Replicate 2

\begin{tabular}{crrrrrr}
\hline Material & Xbar & $\boldsymbol{s}_{\boldsymbol{x}}$ & $\boldsymbol{s}_{\boldsymbol{r}}$ & $\boldsymbol{s}_{\boldsymbol{R}}$ & $\boldsymbol{r}$ & $\boldsymbol{R}$ \\
\hline 1 & 20.5316 & 0.0818 & 0.0467 & 0.0922 & 0.13 & 0.26 \\
2 & 20.2336 & 0.1028 & 0.0607 & 0.1168 & 0.17 & 0.33 \\
\hline
\end{tabular}

XRF Powder Replicate 1

\begin{tabular}{crrrrrr}
\hline Material & Xbar & $\boldsymbol{s}_{\boldsymbol{x}}$ & $\boldsymbol{s}_{\boldsymbol{r}}$ & $\boldsymbol{s}_{\boldsymbol{R}}$ & $\boldsymbol{r}$ & $\boldsymbol{R}$ \\
\hline 1 & 20.6700 & 0.1258 & 0.0561 & 0.1358 & 0.16 & 0.38 \\
2 & 20.1170 & 0.1760 & 0.0538 & 0.1827 & 0.15 & 0.51 \\
\hline
\end{tabular}

XRF Powder, Replicate 2

\begin{tabular}{crrrrrr}
\hline Material & Xbar & $\boldsymbol{s}_{\boldsymbol{x}}$ & $\boldsymbol{s}_{\boldsymbol{r}}$ & $\boldsymbol{s}_{\boldsymbol{R}}$ & $\boldsymbol{r}$ & $\boldsymbol{R}$ \\
\hline 1 & 20.6776 & 0.1255 & 0.0584 & 0.1363 & 0.16 & 0.38 \\
2 & 20.1218 & 0.1756 & 0.0542 & 0.1824 & 0.15 & 0.51 \\
\hline
\end{tabular}

\section{SiO2}

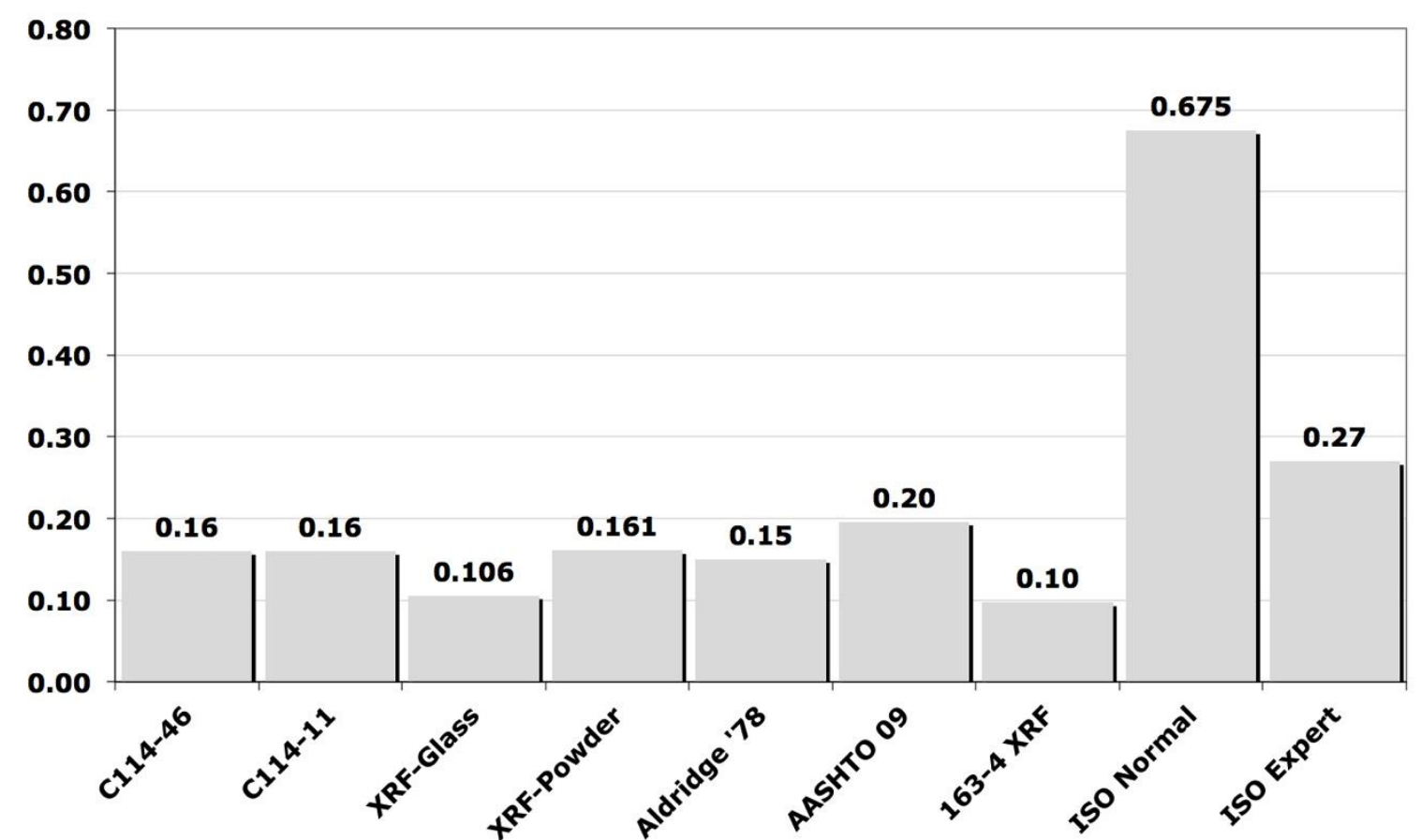

Figure $8 \mathrm{SiO}_{2}$ precision statistics by method with bar chart comparing results to current and past ASTM C114 limits and previous studies on chemical analysis precision as 1-sigma, between lab $\left(\mathbf{S}_{\mathbf{R}}\right)$. 
$\mathrm{Al}_{2} \mathrm{O}_{3}$

Al2O3
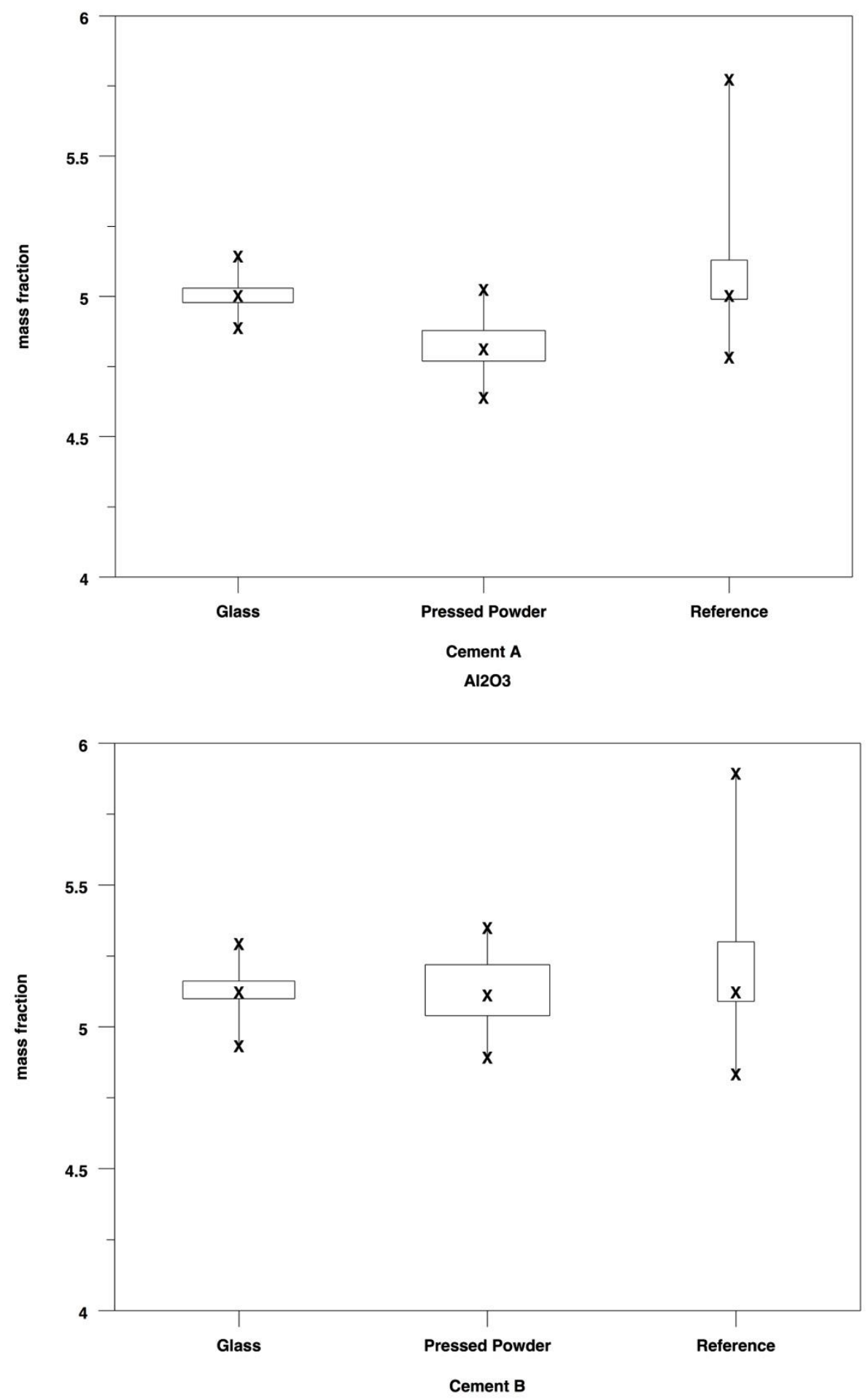

Figure 9 Box plots for $\mathrm{Al}_{2} \mathrm{O}_{3}$ XRF glass and powder, and reference methods. 
$\mathrm{Al}_{2} \mathrm{O}_{3} \mathrm{XRF}$, Cements A B, Glass, Replicate 1, Material: 1

\begin{tabular}{crrrrr}
\hline $\begin{array}{c}\text { Laboratory } \\
\text { Number }\end{array}$ & $\begin{array}{r}\text { Cell } \\
\text { Mean }\end{array}$ & $\begin{array}{r}\text { Cell } \\
\text { SD }\end{array}$ & $\boldsymbol{d}$ & $\boldsymbol{h}$ & $\boldsymbol{k}$ \\
\hline 1 & 4.9298 & 0.0239 & -0.0780 & -1.58 & 1.11 \\
2 & 5.0260 & 0.0114 & 0.0182 & 0.37 & 0.53 \\
3 & 4.9683 & 0.0098 & -0.0395 & -0.80 & 0.46 \\
4 & 4.9777 & 0.0144 & -0.0302 & -0.61 & 0.67 \\
5 & 4.9733 & 0.0266 & -0.0345 & -0.70 & 1.23 \\
6 & 5.1367 & 0.0082 & 0.1288 & 2.60 & 0.38 \\
7 & 5.0002 & 0.0223 & -0.0077 & -0.16 & 1.04 \\
8 & 5.0133 & 0.0163 & 0.0055 & 0.11 & 0.76 \\
9 & 4.9683 & 0.0279 & -0.0395 & -0.80 & 1.29 \\
10 & 4.9450 & 0.0281 & -0.0628 & -1.27 & 1.30 \\
11 & 5.0117 & 0.0214 & 0.0038 & 0.08 & 0.99 \\
12 & 5.0638 & 0.0403 & 0.0560 & 1.13 & 1.87 \\
13 & 4.9883 & 0.0248 & -0.0195 & -0.39 & 1.15 \\
14 & 5.0150 & 0.0105 & 0.0072 & 0.14 & 0.49 \\
15 & 5.0450 & 0.0302 & 0.0372 & 0.75 & 1.40 \\
16 & 5.0492 & 0.0047 & 0.0413 & 0.84 & 0.22 \\
17 & 5.0217 & 0.0075 & 0.0138 & 0.28 & 0.35 \\
\hline
\end{tabular}

Average of cell averages $\quad=\quad 5.00784$

Standard Deviation of cell averages $=0.04946$

Repeatability Standard Deviation $=0.02156$

Reproducibility Standard Deviation $=0.05323$

$\mathrm{h}, \mathrm{k}$ Critical Values $\quad=\quad 2.51000,1.78000$ 
$\mathrm{Al}_{2} \mathrm{O}_{3} \mathrm{XRF}$, Cements A B, Glass, Replicate 1, Material: 2

\begin{tabular}{crrrrr}
\hline $\begin{array}{c}\text { Laboratory } \\
\text { Number }\end{array}$ & $\begin{array}{r}\text { Cell } \\
\text { Mean }\end{array}$ & $\begin{array}{r}\text { Cell } \\
\text { SD }\end{array}$ & $\boldsymbol{d}$ & $\boldsymbol{h}$ & $\boldsymbol{k}$ \\
\hline 1 & 5.0475 & 0.0165 & -0.0825 & -1.45 & 0.74 \\
2 & 5.1378 & 0.0091 & 0.0078 & 0.14 & 0.41 \\
3 & 5.1150 & 0.0084 & -0.0150 & -0.26 & 0.37 \\
4 & 5.1015 & 0.0117 & -0.0285 & -0.50 & 0.52 \\
5 & 5.1133 & 0.0294 & -0.0167 & -0.29 & 1.32 \\
6 & 5.2583 & 0.0172 & 0.1283 & 2.26 & 0.77 \\
7 & 5.1467 & 0.0181 & 0.0167 & 0.29 & 0.81 \\
8 & 5.1233 & 0.0197 & -0.0067 & -0.12 & 0.88 \\
9 & 5.0933 & 0.0301 & -0.0367 & -0.64 & 1.35 \\
10 & 5.0117 & 0.0407 & -0.1183 & -2.08 & 1.82 \\
11 & 5.1283 & 0.0172 & -0.0017 & -0.03 & 0.77 \\
12 & 5.1747 & 0.0234 & 0.0447 & 0.79 & 1.05 \\
13 & 5.0917 & 0.0392 & -0.0383 & -0.67 & 1.76 \\
14 & 5.1433 & 0.0207 & 0.0133 & 0.23 & 0.93 \\
15 & 5.2067 & 0.0197 & 0.0767 & 1.35 & 0.88 \\
16 & 5.1702 & 0.0026 & 0.0402 & 0.71 & 0.12 \\
17 & 5.1467 & 0.0163 & 0.0167 & 0.29 & 0.73 \\
\hline
\end{tabular}

Average of cell averages $\quad=\quad 5.13000$

Standard Deviation of cell averages $=0.05686$

Repeatability Standard Deviation $=0.02232$

Reproducibility Standard Deviation $=0.06040$

$\mathrm{h}, \mathrm{k}$ Critical Values $\quad=\quad 2.51000,1.78000$ 
$\mathrm{Al}_{2} \mathrm{O}_{3} \mathrm{XRF}$, Cements A B, Glass, Replicate 2, Material: 1

\begin{tabular}{crrrrr}
\hline $\begin{array}{c}\text { Laboratory } \\
\text { Number }\end{array}$ & $\begin{array}{c}\text { Cell } \\
\text { Mean }\end{array}$ & $\begin{array}{r}\text { Cell } \\
\text { SD }\end{array}$ & $\boldsymbol{d}$ & $\boldsymbol{h}$ & $\boldsymbol{k}$ \\
\hline 1 & 4.9332 & 0.0270 & -0.0753 & -1.51 & 1.43 \\
2 & 5.0187 & 0.0074 & 0.0102 & 0.20 & 0.39 \\
3 & 4.9767 & 0.0052 & -0.0318 & -0.64 & 0.27 \\
4 & 4.9707 & 0.0107 & -0.0378 & -0.76 & 0.57 \\
5 & 4.9750 & 0.0207 & -0.0335 & -0.67 & 1.10 \\
6 & 5.1350 & 0.0138 & 0.1265 & 2.54 & 0.73 \\
7 & 4.9952 & 0.0200 & -0.0133 & -0.27 & 1.06 \\
8 & 5.0050 & 0.0138 & -0.0035 & -0.07 & 0.73 \\
9 & 4.9567 & 0.0207 & -0.0518 & -1.04 & 1.10 \\
10 & 4.9483 & 0.0256 & -0.0601 & -1.21 & 1.36 \\
11 & 5.0117 & 0.0306 & 0.0032 & 0.06 & 1.63 \\
12 & 5.0805 & 0.0206 & 0.0720 & 1.45 & 1.10 \\
13 & 5.0133 & 0.0121 & 0.0049 & 0.10 & 0.64 \\
14 & 5.0200 & 0.0167 & 0.0115 & 0.23 & 0.89 \\
15 & 5.0467 & 0.0273 & 0.0382 & 0.77 & 1.45 \\
16 & 5.0377 & 0.0099 & 0.0292 & 0.59 & 0.53 \\
17 & 5.0200 & 0.0126 & 0.0115 & 0.23 & 0.67 \\
\hline
\end{tabular}

Average of cell averages $\quad=\quad 5.00848$

Standard Deviation of cell averages $=0.04977$

Repeatability Standard Deviation $=0.01880$

Reproducibility Standard Deviation $=0.05265$

$\mathrm{h}, \mathrm{k}$ Critical Values $\quad=\quad 2.51000,1.78000$ 
$\mathrm{Al}_{2} \mathrm{O}_{3}$ XRF, Cements A B, Glass, Replicate 2, Material: 2

\begin{tabular}{crrrrr}
\hline $\begin{array}{c}\text { Laboratory } \\
\text { Number }\end{array}$ & $\begin{array}{r}\text { Cell } \\
\text { Mean }\end{array}$ & $\begin{array}{r}\text { Cell } \\
\text { SD }\end{array}$ & $\boldsymbol{d}$ & $\boldsymbol{h}$ & $\boldsymbol{k}$ \\
\hline 1 & 5.0528 & 0.0174 & -0.0802 & -1.38 & 0.84 \\
2 & 5.1357 & 0.0082 & 0.0027 & 0.05 & 0.40 \\
3 & 5.1150 & 0.0105 & -0.0180 & -0.31 & 0.51 \\
4 & 5.1038 & 0.0181 & -0.0292 & -0.50 & 0.87 \\
5 & 5.1100 & 0.0283 & -0.0230 & -0.40 & 1.37 \\
6 & 5.2717 & 0.0240 & 0.1387 & 2.39 & 1.16 \\
7 & 5.1485 & 0.0144 & 0.0155 & 0.27 & 0.69 \\
8 & 5.1250 & 0.0138 & -0.0080 & -0.14 & 0.67 \\
9 & 5.0733 & 0.0121 & -0.0597 & -1.03 & 0.58 \\
10 & 5.0300 & 0.0253 & -0.1030 & -1.77 & 1.22 \\
11 & 5.1200 & 0.0141 & -0.0130 & -0.22 & 0.68 \\
12 & 5.1952 & 0.0281 & 0.0622 & 1.07 & 1.36 \\
13 & 5.1050 & 0.0389 & -0.0280 & -0.48 & 1.88 \\
14 & 5.1550 & 0.0302 & 0.0220 & 0.38 & 1.46 \\
15 & 5.2083 & 0.0160 & 0.0753 & 1.30 & 0.77 \\
16 & 5.1635 & 0.0016 & 0.0305 & 0.52 & 0.08 \\
17 & 5.1483 & 0.0160 & 0.0153 & 0.26 & 0.77 \\
\hline
\end{tabular}

Average of cell averages $\quad=\quad 5.13301$

Standard Deviation of cell averages $=0.05808$

Repeatability Standard Deviation $=0.02071$

Reproducibility Standard Deviation $=0.06109$

$\mathrm{h}, \mathrm{k}$ Critical Values $\quad=\quad 2.57000,1.79000$ 
$\mathrm{Al}_{2} \mathrm{O}_{3} \mathrm{XRF}$, Cements A B, Powder, Replicate 1, Material: 1

\begin{tabular}{crrrrr}
\hline $\begin{array}{c}\text { Laboratory } \\
\text { Number }\end{array}$ & $\begin{array}{r}\text { Cell } \\
\text { Mean }\end{array}$ & $\begin{array}{r}\text { Cell } \\
\text { SD }\end{array}$ & $\boldsymbol{d}$ & $\boldsymbol{h}$ & $\boldsymbol{k}$ \\
\hline 1 & 4.7500 & 0.0522 & -0.0713 & -0.92 & 2.18 \\
2 & 4.7317 & 0.0194 & -0.0896 & -1.16 & 0.81 \\
3 & 4.7213 & 0.0305 & -0.0999 & -1.29 & 1.28 \\
4 & 4.8473 & 0.0365 & 0.0261 & 0.34 & 1.53 \\
5 & 4.9483 & 0.0133 & 0.1271 & 1.64 & 0.56 \\
6 & 4.8267 & 0.0186 & 0.0054 & 0.07 & 0.78 \\
7 & 4.7917 & 0.0117 & -0.0296 & -0.38 & 0.49 \\
8 & 4.9850 & 0.0138 & 0.1637 & 2.12 & 0.58 \\
9 & 4.8267 & 0.0082 & 0.0054 & 0.07 & 0.34 \\
10 & 4.8400 & 0.0167 & 0.0187 & 0.24 & 0.70 \\
11 & 4.8853 & 0.0066 & 0.0641 & 0.83 & 0.27 \\
13 & 4.7647 & 0.0155 & -0.0566 & -0.73 & 0.65 \\
14 & 4.7867 & 0.0250 & -0.0346 & -0.45 & 1.05 \\
15 & 4.7527 & 0.0166 & -0.0686 & -0.89 & 0.70 \\
16 & 4.9555 & 0.0405 & 0.1342 & 1.74 & 1.69 \\
17 & 4.7783 & 0.0223 & -0.0429 & -0.56 & 0.93 \\
18 & 4.8283 & 0.0075 & 0.0071 & 0.09 & 0.32 \\
19 & 4.8280 & 0.0261 & 0.0067 & 0.09 & 1.09 \\
20 & 4.8083 & 0.0306 & -0.0129 & -0.17 & 1.28 \\
21 & 4.8867 & 0.0175 & 0.0654 & 0.85 & 0.73 \\
22 & 4.7033 & 0.0109 & -0.1179 & -1.53 & 0.45 \\
\hline
\end{tabular}

Average of cell averages $\quad=\quad 4.82126$

Standard Deviation of cell averages $=0.07731$

Repeatability Standard Deviation $=0.02388$

Reproducibility Standard Deviation $=0.08032$

$\mathrm{h}, \mathrm{k}$ Critical Value $\quad=\quad 2.57000,1.79000$ 
$\mathrm{Al}_{2} \mathrm{O}_{3}$ XRF, Cements A B, Powder, Replicate 1, Material: 2

\begin{tabular}{crrrrr}
\hline $\begin{array}{c}\text { Laboratory } \\
\text { Number }\end{array}$ & $\begin{array}{r}\text { Cell } \\
\text { Mean }\end{array}$ & $\begin{array}{r}\text { Cell } \\
\text { SD }\end{array}$ & $\boldsymbol{d}$ & $\boldsymbol{h}$ & $\boldsymbol{k}$ \\
\hline 1 & 4.9433 & 0.0234 & -0.1858 & -1.68 & 1.20 \\
2 & 5.2250 & 0.0084 & 0.0958 & 0.87 & 0.43 \\
3 & 5.3067 & 0.0207 & 0.1775 & 1.61 & 1.06 \\
4 & 5.1215 & 0.0196 & -0.0077 & -0.07 & 1.00 \\
5 & 5.2733 & 0.0175 & 0.1442 & 1.31 & 0.90 \\
6 & 5.0650 & 0.0105 & -0.0642 & -0.58 & 0.54 \\
7 & 5.0267 & 0.0103 & -0.1025 & -0.93 & 0.53 \\
8 & 5.2833 & 0.0175 & 0.1542 & 1.40 & 0.90 \\
9 & 5.1367 & 0.0137 & 0.0075 & 0.07 & 0.70 \\
10 & 5.1883 & 0.0331 & 0.0592 & 0.54 & 1.69 \\
11 & 5.0728 & 0.0284 & -0.0563 & -0.51 & 1.45 \\
13 & 5.3123 & 0.0125 & 0.1832 & 1.66 & 0.64 \\
14 & 5.0383 & 0.0194 & -0.0908 & -0.82 & 0.99 \\
15 & 4.9863 & 0.0263 & -0.1428 & -1.29 & 1.35 \\
16 & 5.2137 & 0.0128 & 0.0845 & 0.77 & 0.66 \\
17 & 5.1367 & 0.0186 & 0.0075 & 0.07 & 0.95 \\
18 & 5.0383 & 0.0214 & -0.0908 & -0.82 & 1.09 \\
19 & 5.0770 & 0.0188 & -0.0522 & -0.47 & 0.96 \\
20 & 5.0733 & 0.0137 & -0.0558 & -0.51 & 0.70 \\
21 & 5.1883 & 0.0286 & 0.0592 & 0.54 & 1.46 \\
22 & 5.0055 & 0.0116 & -0.1237 & -1.12 & 0.59 \\
\hline
\end{tabular}

Average of cell averages $\quad=\quad 5.12917$

Standard Deviation of cell averages $=0.11033$

Repeatability Standard Deviation $=0.01956$

Reproducibility Standard Deviation $=0.11177$

$\mathrm{h}, \mathrm{k}$ Critical Value $\quad=\quad 2.57000,1.79000$ 
$\mathrm{Al}_{2} \mathrm{O}_{3}$ XRF, Cements A B, Powder, Replicate 2, Material: 1

\begin{tabular}{llllrl}
\hline Laboratory & Cell & Cell & & \\
Number & Mean & SD & \multicolumn{1}{l}{$d$} & \multicolumn{1}{l}{$h$} \\
\hline 1 & 4.7600 & 0.0460 & -0.0667 & -0.87 & 1.88 \\
2 & 4.7333 & 0.0207 & -0.0933 & -1.22 & 0.85 \\
3 & 4.7257 & 0.0414 & -0.1010 & -1.32 & 1.70 \\
4 & 4.8528 & 0.0411 & 0.0262 & 0.34 & 1.68 \\
5 & 4.9467 & 0.0121 & 0.1200 & 1.57 & 0.50 \\
6 & 4.8350 & 0.0243 & 0.0083 & 0.11 & 0.99 \\
7 & 4.7933 & 0.0082 & -0.0333 & -0.44 & 0.33 \\
8 & 4.9883 & 0.0133 & 0.1617 & 2.12 & 0.54 \\
9 & 4.8333 & 0.0082 & 0.0067 & 0.09 & 0.33 \\
10 & 4.8467 & 0.0197 & 0.0200 & 0.26 & 0.80 \\
11 & 4.8878 & 0.0088 & 0.0612 & 0.80 & 0.36 \\
13 & 4.7662 & 0.0134 & -0.0605 & -0.79 & 0.55 \\
14 & 4.7783 & 0.0214 & -0.0483 & -0.63 & 0.87 \\
15 & 4.7917 & 0.0085 & -0.0350 & -0.46 & 0.35 \\
16 & 4.9592 & 0.0395 & 0.1325 & 1.74 & 1.62 \\
17 & 4.7883 & 0.0204 & -0.0383 & -0.50 & 0.84 \\
18 & 4.8333 & 0.0103 & 0.0067 & 0.09 & 0.42 \\
19 & 4.8313 & 0.0273 & 0.0047 & 0.06 & 1.12 \\
20 & 4.8233 & 0.0314 & -0.0033 & -0.04 & 1.29 \\
21 & 4.8867 & 0.0207 & 0.0600 & 0.79 & 0.85 \\
22 & 4.6983 & 0.0113 & -0.1283 & -1.68 & 0.46 \\
\hline
\end{tabular}

Average of cell averages $\quad=\quad 4.82665$

Standard Deviation of cell averages $=0.07627$

Repeatability Standard Deviation $=0.02443$

Reproducibility Standard Deviation $=0.07946$

$\mathrm{h}, \mathrm{k}$ Critical Value $\quad=\quad 2.57000,1.79000$ 
$\mathrm{Al}_{2} \mathrm{O}_{3}$ XRF, Cements A B, Powder, Replicate 2, Material: 2

\begin{tabular}{crrrrr}
\hline $\begin{array}{c}\text { Laboratory } \\
\text { Number }\end{array}$ & $\begin{array}{r}\text { Cell } \\
\text { Mean }\end{array}$ & $\begin{array}{r}\text { Cell } \\
\text { SD }\end{array}$ & $\boldsymbol{d}$ & $\boldsymbol{h}$ & $\boldsymbol{k}$ \\
\hline 1 & 4.9483 & 0.0279 & -0.1837 & -1.72 & 1.38 \\
2 & 5.2117 & 0.0183 & 0.0796 & 0.75 & 0.91 \\
3 & 5.3157 & 0.0284 & 0.1836 & 1.72 & 1.40 \\
4 & 5.1247 & 0.0222 & -0.0074 & -0.07 & 1.10 \\
5 & 5.2733 & 0.0103 & 0.1413 & 1.33 & 0.51 \\
6 & 5.0650 & 0.0138 & -0.0671 & -0.63 & 0.68 \\
7 & 5.0250 & 0.0105 & -0.1071 & -1.00 & 0.52 \\
8 & 5.2783 & 0.0147 & 0.1463 & 1.37 & 0.73 \\
9 & 5.1400 & 0.0110 & 0.0079 & 0.07 & 0.54 \\
10 & 5.1867 & 0.0344 & 0.0546 & 0.51 & 1.70 \\
11 & 5.0717 & 0.0207 & -0.0604 & -0.57 & 1.02 \\
13 & 5.3052 & 0.0118 & 0.1731 & 1.62 & 0.58 \\
14 & 5.0367 & 0.0207 & -0.0954 & -0.89 & 1.02 \\
15 & 5.0317 & 0.0171 & -0.1004 & -0.94 & 0.85 \\
16 & 5.2122 & 0.0111 & 0.0801 & 0.75 & 0.55 \\
17 & 5.1333 & 0.0175 & 0.0013 & 0.01 & 0.86 \\
18 & 5.0333 & 0.0197 & -0.0987 & -0.93 & 0.97 \\
19 & 5.0877 & 0.0334 & -0.0444 & -0.42 & 1.65 \\
20 & 5.1017 & 0.0075 & -0.0304 & -0.29 & 0.37 \\
21 & 5.1917 & 0.0279 & 0.0596 & 0.56 & 1.38 \\
22 & 4.9995 & 0.0149 & -0.1326 & -1.24 & 0.74 \\
\hline
\end{tabular}

Average of cell averages $\quad=\quad 5.13206$

Standard Deviation of cell averages $=0.10658$

Repeatability Standard Deviation $=0.02025$

Reproducibility Standard Deviation $=0.10817$

$\mathrm{h}, \mathrm{k}$ Critical Value $\quad=\quad 2.57000,1.79000$ 
Table 4 Precision Statistics $\mathrm{Al}_{2} \mathrm{O}_{3}$ by XRF glass and powder preparations for replicates 1 and 2

$\mathrm{Al}_{2} \mathrm{O}_{3}$ Glass, Replicate 1

\begin{tabular}{lllllll}
\hline Material & Xbar & $\boldsymbol{s}_{\boldsymbol{x}}$ & $\boldsymbol{s}_{\boldsymbol{r}}$ & $\boldsymbol{s}_{\boldsymbol{R}}$ & $\boldsymbol{r}$ & $\boldsymbol{R}$ \\
\hline 1 & 5.0078 & 0.0495 & 0.0216 & 0.0532 & 0.06 & 0.15 \\
2 & 5.1300 & 0.0569 & 0.0223 & 0.0604 & 0.06 & 0.17 \\
\hline
\end{tabular}

$\mathrm{Al}_{2} \mathrm{O}_{3}$ Glass, Replicate 2

\begin{tabular}{lllllll}
\hline Material & Xbar & $\boldsymbol{s}_{\boldsymbol{x}}$ & $\boldsymbol{s}_{\boldsymbol{r}}$ & $\boldsymbol{s}_{\boldsymbol{R}}$ & $\boldsymbol{r}$ & $\boldsymbol{R}$ \\
\hline 1 & 5.0085 & 0.0498 & 0.0188 & 0.0526 & 0.05 & 0.15 \\
2 & 5.1330 & 0.0581 & 0.0207 & 0.0611 & 0.06 & 0.17 \\
\hline
\end{tabular}

$\mathrm{Al}_{2} \mathrm{O}_{3}$ Powder, Replicate 1

\begin{tabular}{lllllll}
\hline Material & Xbar & $\boldsymbol{s}_{\boldsymbol{x}}$ & $\boldsymbol{s}_{\boldsymbol{r}}$ & $\boldsymbol{s}_{\boldsymbol{R}}$ & $\boldsymbol{r}$ & $\boldsymbol{R}$ \\
\hline 1 & 4.8213 & 0.0773 & 0.0239 & 0.0803 & 0.07 & 0.22 \\
2 & 5.1292 & 0.1103 & 0.0196 & 0.1118 & 0.05 & 0.31 \\
\hline
\end{tabular}

$\mathrm{Al}_{2} \mathrm{O}_{3}$ Powder, Replicate 2

\begin{tabular}{lllllll}
\hline Material & Xbar & $\boldsymbol{s}_{\boldsymbol{x}}$ & $\boldsymbol{s}_{\boldsymbol{r}}$ & $\boldsymbol{s}_{\boldsymbol{R}}$ & $\boldsymbol{r}$ & $\boldsymbol{R}$ \\
\hline 1 & 4.8267 & 0.0763 & 0.0244 & 0.0795 & 0.07 & 0.22 \\
2 & 5.1321 & 0.1066 & 0.0203 & 0.1082 & 0.06 & 0.30 \\
\hline
\end{tabular}

\section{Al203}

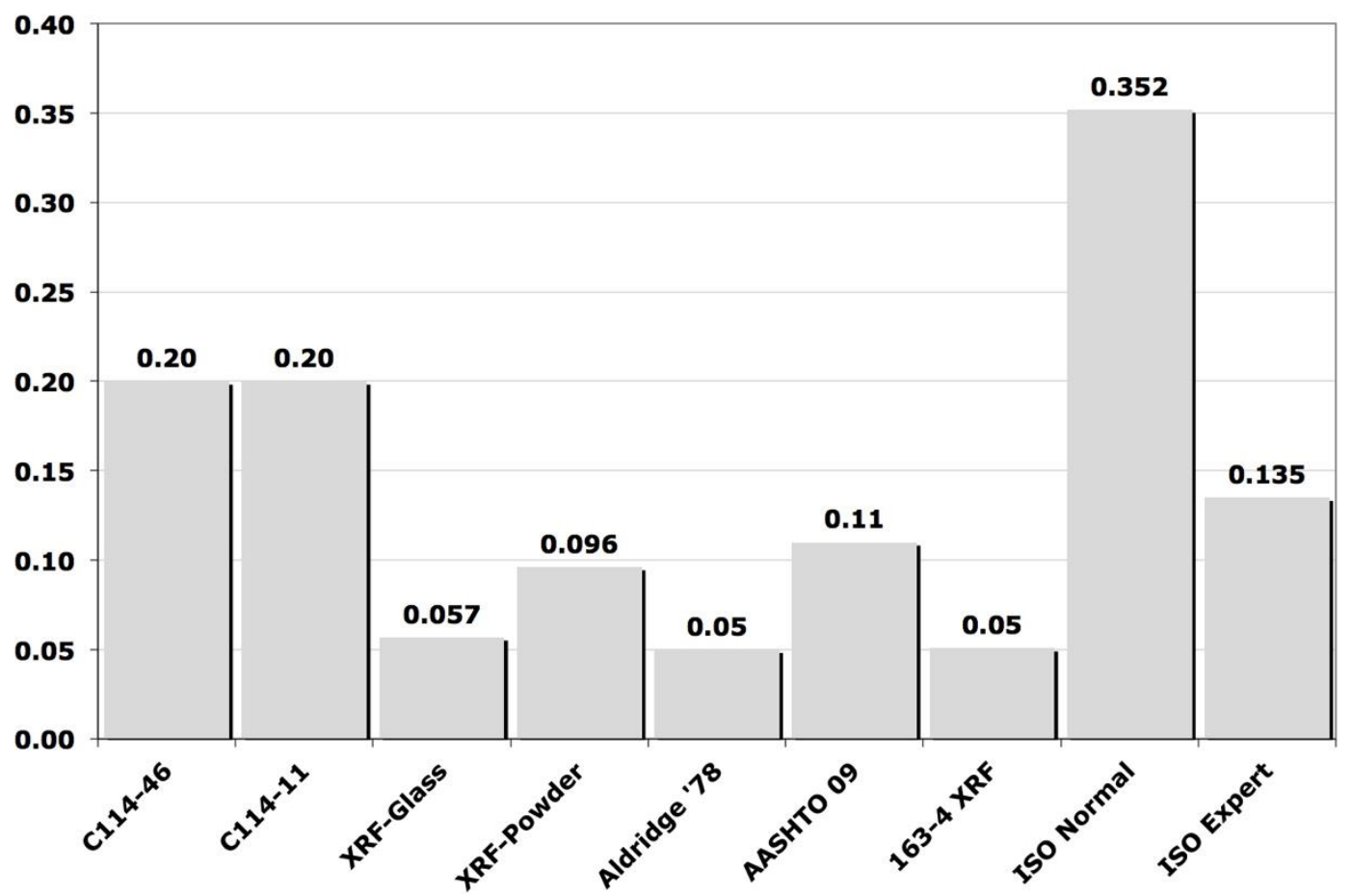

Figure $10 \mathrm{Al}_{2} \mathrm{O}_{2}$ precision statistics by method with bar chart comparing results to current and past ASTM C114 limits and previous studies on chemical analysis precision as 1-sigma, between lab $\left(S_{R}\right)$. 
$\mathrm{Fe}_{2} \mathrm{O}_{3}$

Fe2O3
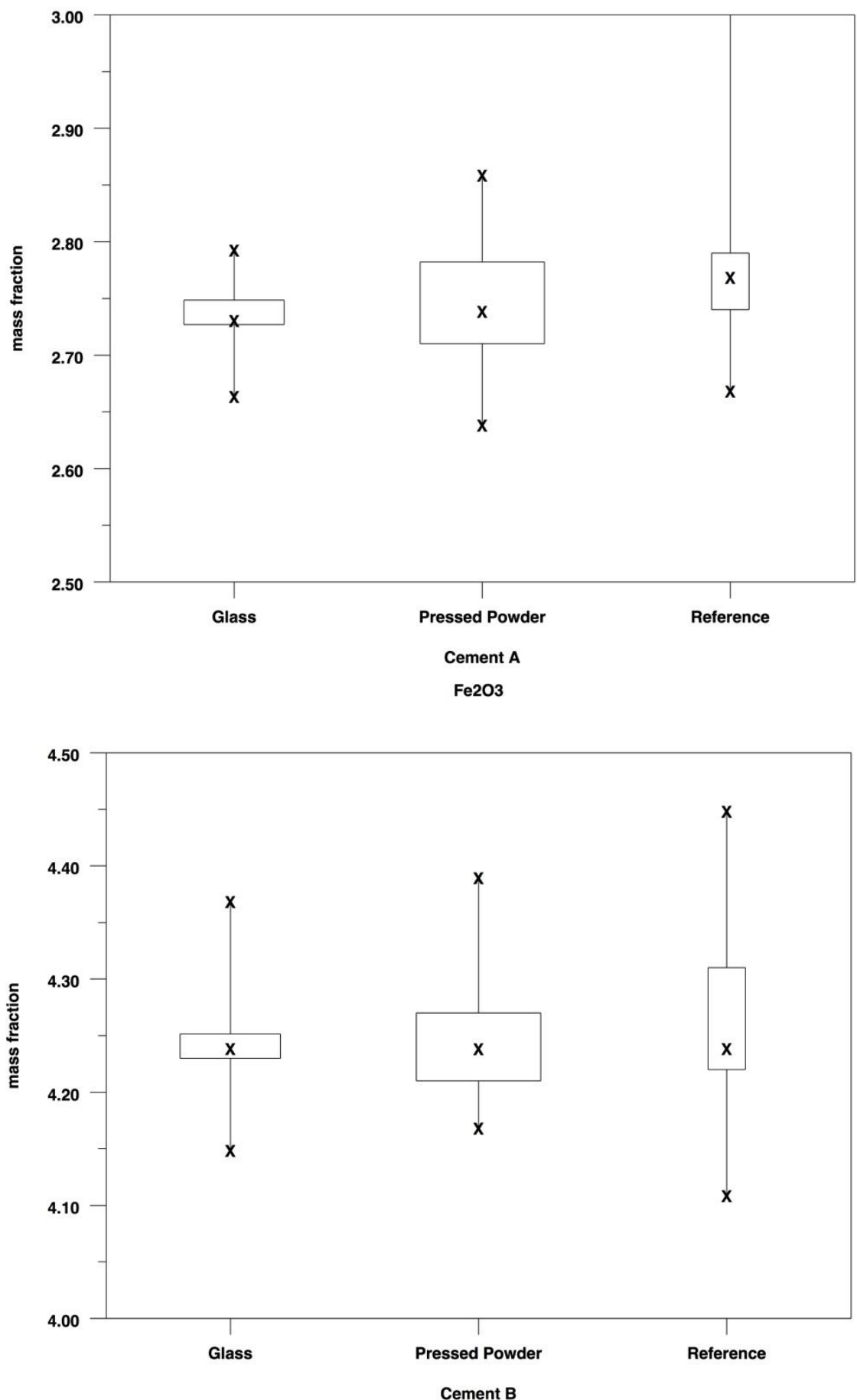

Figure 11 Box plots for $\mathrm{Fe}_{2} \mathrm{O}_{3}$ XRF glass and powder, and reference methods. 
$\mathrm{Fe}_{2} \mathrm{O}_{3}$ XRF, Cements A B, glass, Replicate 1, Material: 1

\begin{tabular}{crrrrr}
\hline $\begin{array}{c}\text { Laboratory } \\
\text { Number }\end{array}$ & $\begin{array}{r}\text { Cell } \\
\text { Mean }\end{array}$ & $\begin{array}{r}\text { Cell } \\
\text { SD }\end{array}$ & $\boldsymbol{d}$ & $\boldsymbol{h}$ & $\boldsymbol{k}$ \\
\hline 1 & 2.7280 & 0.0158 & -0.0083 & -0.29 & 1.15 \\
2 & 2.7313 & 0.0338 & -0.0050 & -0.18 & 2.46 \\
3 & 2.7287 & 0.0034 & -0.0077 & -0.27 & 0.25 \\
4 & 2.7317 & 0.0041 & -0.0047 & -0.16 & 0.30 \\
5 & 2.7295 & 0.0025 & -0.0068 & -0.24 & 0.18 \\
6 & 2.7333 & 0.0052 & -0.0030 & -0.11 & 0.38 \\
7 & 2.6900 & 0.0089 & -0.0463 & -1.64 & 0.65 \\
8 & 2.7390 & 0.0064 & 0.0027 & 0.09 & 0.47 \\
9 & 2.7850 & 0.0055 & 0.0487 & 1.72 & 0.40 \\
10 & 2.6900 & 0.0297 & -0.0463 & -1.64 & 2.16 \\
11 & 2.7383 & 0.0075 & 0.0020 & 0.07 & 0.55 \\
12 & 2.7750 & 0.0130 & 0.0387 & 1.37 & 0.94 \\
13 & 2.7283 & 0.0098 & -0.0080 & -0.28 & 0.71 \\
14 & 2.7883 & 0.0041 & 0.0520 & 1.84 & 0.30 \\
15 & 2.7283 & 0.0041 & -0.0080 & -0.28 & 0.30 \\
\hline
\end{tabular}

Average of cell averages $\quad=\quad 2.73632$

Standard Deviation of cell averages $=0.02832$

Repeatability Standard Deviation $=0.01376$

Reproducibility Standard Deviation $=0.03098$

$\mathrm{h}$, k Critical Values $\quad=\quad 2.47000,1.77000$ 
$\mathrm{Fe}_{2} \mathrm{O}_{3}$ XRF, Cements A B, glass, Replicate 1, Material: 2

\begin{tabular}{crrrrr}
\hline $\begin{array}{c}\text { Laboratory } \\
\text { Number }\end{array}$ & $\begin{array}{r}\text { Cell } \\
\text { Mean }\end{array}$ & $\begin{array}{r}\text { Cell } \\
\text { SD }\end{array}$ & $\boldsymbol{d}$ & $\boldsymbol{h}$ & $\boldsymbol{k}$ \\
\hline 1 & 4.2225 & 0.0081 & -0.0176 & -0.60 & 0.75 \\
2 & 4.2363 & 0.0259 & -0.0038 & -0.13 & 2.40 \\
3 & 4.2460 & 0.0037 & 0.0059 & 0.20 & 0.35 \\
4 & 4.2167 & 0.0082 & -0.0234 & -0.80 & 0.76 \\
5 & 4.2398 & 0.0052 & -0.0003 & -0.01 & 0.49 \\
6 & 4.2517 & 0.0075 & 0.0116 & 0.40 & 0.70 \\
7 & 4.1683 & 0.0117 & -0.0718 & -2.46 & 1.08 \\
8 & 4.2408 & 0.0063 & 0.0007 & 0.02 & 0.58 \\
9 & 4.3000 & 0.0063 & 0.0599 & 2.06 & 0.59 \\
10 & 4.2583 & 0.0194 & 0.0182 & 0.63 & 1.80 \\
11 & 4.2300 & 0.0063 & -0.0101 & -0.35 & 0.59 \\
12 & 4.2528 & 0.0095 & 0.0127 & 0.44 & 0.88 \\
13 & 4.2300 & 0.0089 & -0.0101 & -0.35 & 0.83 \\
14 & 4.2767 & 0.0052 & 0.0366 & 1.25 & 0.48 \\
15 & 4.2317 & 0.0041 & -0.0084 & -0.29 & 0.38 \\
\hline
\end{tabular}

Average of cell averages $\quad=\quad 4.24011$

Standard Deviation of cell averages $=0.02914$

Repeatability Standard Deviation $=0.01079$

Reproducibility Standard Deviation $=0.03076$

$\mathrm{h}, \mathrm{k}$ Critical Values $\quad=\quad 2.47000,1.77000$ 
$\mathrm{Fe}_{2} \mathrm{O}_{3}$ XRF, Cements A B, glass, Replicate 2, Material: 1

\begin{tabular}{crrrrr}
\hline $\begin{array}{c}\text { Laboratory } \\
\text { Number }\end{array}$ & $\begin{array}{r}\text { Cell } \\
\text { Mean }\end{array}$ & $\begin{array}{r}\text { Cell } \\
\text { SD }\end{array}$ & $\boldsymbol{d}$ & $\boldsymbol{h}$ & $\boldsymbol{k}$ \\
\hline 1 & 2.7250 & 0.0059 & -0.0121 & -0.42 & 0.54 \\
2 & 2.7333 & 0.0335 & -0.0038 & -0.13 & 3.07 \\
3 & 2.7295 & 0.0028 & -0.0076 & -0.27 & 0.26 \\
4 & 2.7317 & 0.0041 & -0.0055 & -0.19 & 0.37 \\
5 & 2.7313 & 0.0022 & -0.0058 & -0.20 & 0.20 \\
6 & 2.7367 & 0.0052 & -0.0005 & -0.02 & 0.47 \\
7 & 2.6917 & 0.0075 & -0.0455 & -1.59 & 0.69 \\
8 & 2.7423 & 0.0044 & 0.0052 & 0.18 & 0.40 \\
9 & 2.7850 & 0.0055 & 0.0479 & 1.67 & 0.50 \\
10 & 2.6867 & 0.0082 & -0.0505 & -1.76 & 0.75 \\
11 & 2.7367 & 0.0121 & -0.0005 & -0.02 & 1.11 \\
12 & 2.7790 & 0.0097 & 0.0419 & 1.46 & 0.89 \\
13 & 2.7300 & 0.0089 & -0.0071 & -0.25 & 0.82 \\
14 & 2.7867 & 0.0052 & 0.0495 & 1.73 & 0.47 \\
15 & 2.7317 & 0.0075 & -0.0055 & -0.19 & 0.69 \\
\hline
\end{tabular}

Average of cell averages $\quad=\quad 2.73714$

Standard Deviation of cell averages $=0.02866$

Repeatability Standard Deviation $=0.01091$

Reproducibility Standard Deviation $=0.03035$

$\mathrm{h}, \mathrm{k}$ Critical Values $\quad=\quad 2.47000,1.77000$ 
$\mathrm{Fe}_{2} \mathrm{O}_{3} \mathrm{XRF}$, Cements A B, glass, Replicate 2, Material: 2

\begin{tabular}{crrrrr}
\hline $\begin{array}{c}\text { Laboratory } \\
\text { Number }\end{array}$ & $\begin{array}{r}\text { Cell } \\
\text { Mean }\end{array}$ & $\begin{array}{r}\text { Cell } \\
\text { SD }\end{array}$ & $\boldsymbol{d}$ & $\boldsymbol{h}$ & $\boldsymbol{k}$ \\
\hline 1 & 4.2173 & 0.0096 & -0.0225 & -0.73 & 0.55 \\
2 & 4.2268 & 0.0286 & -0.0130 & -0.42 & 1.65 \\
3 & 4.2448 & 0.0038 & 0.0050 & 0.16 & 0.22 \\
4 & 4.2150 & 0.0084 & -0.0249 & -0.81 & 0.48 \\
5 & 4.2385 & 0.0012 & -0.0014 & -0.04 & 0.07 \\
6 & 4.2483 & 0.0075 & 0.0085 & 0.27 & 0.43 \\
7 & 4.1667 & 0.0151 & -0.0732 & -2.37 & 0.87 \\
8 & 4.2450 & 0.0068 & 0.0051 & 0.17 & 0.39 \\
9 & 4.2983 & 0.0075 & 0.0585 & 1.89 & 0.43 \\
10 & 4.2700 & 0.0514 & 0.0301 & 0.98 & 2.96 \\
11 & 4.2317 & 0.0133 & -0.0082 & -0.27 & 0.77 \\
12 & 4.2603 & 0.0080 & 0.0205 & 0.66 & 0.46 \\
13 & 4.2283 & 0.0098 & -0.0115 & -0.37 & 0.57 \\
14 & 4.2783 & 0.0041 & 0.0385 & 1.25 & 0.24 \\
15 & 4.2283 & 0.0117 & -0.0115 & -0.37 & 0.67 \\
\hline
\end{tabular}

Average of cell averages $\quad=\quad 4.23986$

Standard Deviation of cell averages $=0.03086$

Repeatability Standard Deviation $=0.01734$

Reproducibility Standard Deviation $=0.03469$

$\mathrm{h}, \mathrm{k}$ Critical Values $\quad=\quad 2.47000,1.77000$ 
$\mathrm{Fe}_{2} \mathrm{O}_{3}$ XRF, Cements A B, Powder, Replicate 1, Material: 1

\begin{tabular}{crrrrr}
\hline $\begin{array}{c}\text { Laboratory } \\
\text { Number }\end{array}$ & $\begin{array}{r}\text { Cell } \\
\text { Mean }\end{array}$ & $\begin{array}{r}\text { Cell } \\
\text { SD }\end{array}$ & $\boldsymbol{d}$ & $\boldsymbol{h}$ & $\boldsymbol{k}$ \\
\hline 1 & 2.6600 & 0.0063 & -0.0844 & -1.92 & 0.50 \\
2 & 2.7083 & 0.0194 & -0.0360 & -0.82 & 1.53 \\
4 & 2.7218 & 0.0212 & -0.0225 & -0.51 & 1.67 \\
5 & 2.7383 & 0.0041 & -0.0060 & -0.14 & 0.32 \\
6 & 2.7500 & 0.0063 & 0.0056 & 0.13 & 0.50 \\
7 & 2.7233 & 0.0052 & -0.0210 & -0.48 & 0.41 \\
8 & 2.8317 & 0.0133 & 0.0873 & 1.99 & 1.05 \\
9 & 2.7833 & 0.0327 & 0.0390 & 0.89 & 2.58 \\
10 & 2.8067 & 0.0082 & 0.0623 & 1.42 & 0.64 \\
11 & 2.7887 & 0.0085 & 0.0443 & 1.01 & 0.67 \\
12 & 2.6765 & 0.0098 & -0.0679 & -1.54 & 0.77 \\
13 & 2.7270 & 0.0095 & -0.0174 & -0.40 & 0.75 \\
14 & 2.7685 & 0.0164 & 0.0241 & 0.55 & 1.29 \\
15 & 2.7200 & 0.0063 & -0.0244 & -0.55 & 0.50 \\
16 & 2.7817 & 0.0041 & 0.0373 & 0.85 & 0.32 \\
17 & 2.7066 & 0.0169 & -0.0377 & -0.86 & 1.33 \\
18 & 2.7468 & 0.0103 & 0.0025 & 0.06 & 0.82 \\
19 & 2.7117 & 0.0160 & -0.0327 & -0.74 & 1.26 \\
20 & 2.7967 & 0.0052 & 0.0523 & 1.19 & 0.41 \\
21 & 2.7513 & 0.0065 & 0.0070 & 0.16 & 0.51 \\
22 & 2.7117 & 0.0041 & -0.0327 & -0.74 & 0.32 \\
23 & 2.7100 & 0.0063 & -0.0344 & -0.78 & 0.50 \\
24 & 2.8000 & 0.0089 & 0.0556 & 1.27 & 0.71 \\
\hline & & & & &
\end{tabular}

Average of cell averages $\quad=\quad 2.74438$

Standard Deviation of cell averages $=0.04396$

Repeatability Standard Deviation $\quad=0.01268$

Reproducibility Standard Deviation $=0.04546$

$\mathrm{h}, \mathrm{k}$ Critical Values $\quad=\quad 2.59000,1.79000$ 
$\mathrm{Fe}_{2} \mathrm{O}_{3}$ XRF, Cements A B, Powder, Replicate 1, Material: 2

\begin{tabular}{crrrrr}
\hline $\begin{array}{c}\text { Laboratory } \\
\text { Number }\end{array}$ & $\begin{array}{r}\text { Cell } \\
\text { Mean }\end{array}$ & $\begin{array}{r}\text { Cell } \\
\text { SD }\end{array}$ & $\boldsymbol{d}$ & $\boldsymbol{h}$ & $\boldsymbol{k}$ \\
\hline 1 & 4.2567 & 0.0137 & 0.0079 & 0.19 & 0.73 \\
2 & 4.2633 & 0.0532 & 0.0146 & 0.35 & 2.84 \\
4 & 4.2250 & 0.0246 & -0.0238 & -0.56 & 1.31 \\
5 & 4.2333 & 0.0103 & -0.0154 & -0.37 & 0.55 \\
6 & 4.2450 & 0.0055 & -0.0038 & -0.09 & 0.29 \\
7 & 4.2500 & 0.0089 & 0.0012 & 0.03 & 0.48 \\
8 & 4.3333 & 0.0082 & 0.0846 & 2.00 & 0.44 \\
9 & 4.2533 & 0.0175 & 0.0046 & 0.11 & 0.93 \\
10 & 4.2917 & 0.0075 & 0.0429 & 1.02 & 0.40 \\
11 & 4.3159 & 0.0110 & 0.0672 & 1.59 & 0.58 \\
12 & 4.1978 & 0.0062 & -0.0509 & -1.21 & 0.33 \\
13 & 4.2055 & 0.0140 & -0.0433 & -1.02 & 0.75 \\
14 & 4.1987 & 0.0100 & -0.0501 & -1.19 & 0.53 \\
15 & 4.2600 & 0.0237 & 0.0113 & 0.27 & 1.26 \\
16 & 4.2717 & 0.0147 & 0.0229 & 0.54 & 0.79 \\
17 & 4.2046 & 0.0137 & -0.0442 & -1.05 & 0.73 \\
18 & 4.3403 & 0.0450 & 0.0916 & 2.17 & 2.40 \\
19 & 4.2067 & 0.0052 & -0.0421 & -1.00 & 0.28 \\
20 & 4.2683 & 0.0041 & 0.0196 & 0.46 & 0.22 \\
21 & 4.2651 & 0.0029 & 0.0163 & 0.39 & 0.16 \\
22 & 4.2100 & 0.0063 & -0.0388 & -0.92 & 0.34 \\
23 & 4.2067 & 0.0121 & -0.0421 & -1.00 & 0.65 \\
24 & 4.2183 & 0.0117 & -0.0304 & -0.72 & 0.62 \\
\hline & & & & &
\end{tabular}

Average of cell averages $\quad=\quad 4.24875$

Standard Deviation of cell averages $=0.04222$

Repeatability Standard Deviation $=0.01875$

Reproducibility Standard Deviation $=0.04555$

$\mathrm{h}, \mathrm{k}$ Critical Values $\quad=\quad 2.59000,1.79000$ 
$\mathrm{Fe}_{2} \mathrm{O}_{3}$ XRF, Cements A B, Powder, Replicate 2, Material: 1

\begin{tabular}{crrrrr}
\hline $\begin{array}{c}\text { Laboratory } \\
\text { Number }\end{array}$ & $\begin{array}{r}\text { Cell } \\
\text { Mean }\end{array}$ & $\begin{array}{r}\text { Cell } \\
\text { SD }\end{array}$ & $\boldsymbol{d}$ & $\boldsymbol{h}$ & $\boldsymbol{k}$ \\
\hline 1 & 2.6533 & 0.0082 & -0.0909 & -2.03 & 0.58 \\
2 & 2.6933 & 0.0333 & -0.0509 & -1.13 & 2.37 \\
4 & 2.7228 & 0.0212 & -0.0214 & -0.48 & 1.51 \\
5 & 2.7400 & 0.0063 & -0.0042 & -0.09 & 0.45 \\
6 & 2.7467 & 0.0103 & 0.0025 & 0.05 & 0.74 \\
7 & 2.7350 & 0.0105 & -0.0092 & -0.21 & 0.75 \\
8 & 2.8317 & 0.0160 & 0.0875 & 1.95 & 1.14 \\
9 & 2.7750 & 0.0295 & 0.0308 & 0.69 & 2.10 \\
10 & 2.8067 & 0.0082 & 0.0625 & 1.39 & 0.58 \\
11 & 2.7945 & 0.0105 & 0.0503 & 1.12 & 0.75 \\
12 & 2.6788 & 0.0051 & -0.0654 & -1.46 & 0.36 \\
13 & 2.7258 & 0.0109 & -0.0184 & -0.41 & 0.78 \\
14 & 2.7705 & 0.0165 & 0.0263 & 0.59 & 1.18 \\
15 & 2.7217 & 0.0075 & -0.0225 & -0.50 & 0.54 \\
16 & 2.7850 & 0.0084 & 0.0408 & 0.91 & 0.60 \\
17 & 2.7064 & 0.0173 & -0.0378 & -0.84 & 1.23 \\
18 & 2.7480 & 0.0097 & 0.0038 & 0.08 & 0.69 \\
19 & 2.7117 & 0.0160 & -0.0325 & -0.73 & 1.14 \\
20 & 2.7950 & 0.0055 & 0.0508 & 1.13 & 0.39 \\
21 & 2.7465 & 0.0048 & 0.0023 & 0.05 & 0.34 \\
22 & 2.7117 & 0.0041 & -0.0325 & -0.73 & 0.29 \\
23 & 2.7150 & 0.0055 & -0.0292 & -0.65 & 0.39 \\
24 & 2.8017 & 0.0075 & 0.0575 & 1.28 & 0.54 \\
\hline & & & & &
\end{tabular}

Average of cell averages $\quad=\quad 2.74421$

Standard Deviation of cell averages $=0.04483$

Repeatability Standard Deviation $=0.01404$

Reproducibility Standard Deviation $=0.04662$

$\mathrm{h}, \mathrm{k}$ Critical Values $\quad=\quad 1.79000,2.59000$ 
$\mathrm{Fe}_{2} \mathrm{O}_{3}$ XRF, Cements A B, Powder, Replicate 2, Material: 2

\begin{tabular}{crrrrr}
\hline $\begin{array}{c}\text { Laboratory } \\
\text { Number }\end{array}$ & $\begin{array}{r}\text { Cell } \\
\text { Mean }\end{array}$ & $\begin{array}{r}\text { Cell } \\
\text { SD }\end{array}$ & $\boldsymbol{d}$ & $\boldsymbol{h}$ & $\boldsymbol{k}$ \\
\hline 1 & 4.2600 & 0.0167 & 0.0125 & 0.29 & 0.92 \\
2 & 4.2683 & 0.0504 & 0.0208 & 0.48 & 2.78 \\
4 & 4.2227 & 0.0198 & -0.0248 & -0.57 & 1.10 \\
5 & 4.2333 & 0.0103 & -0.0142 & -0.33 & 0.57 \\
6 & 4.2483 & 0.0075 & 0.0008 & 0.02 & 0.42 \\
7 & 4.2433 & 0.0082 & -0.0042 & -0.10 & 0.45 \\
8 & 4.3350 & 0.0055 & 0.0875 & 2.02 & 0.30 \\
9 & 4.2017 & 0.0223 & -0.0458 & -1.06 & 1.23 \\
10 & 4.2950 & 0.0055 & 0.0475 & 1.10 & 0.30 \\
11 & 4.3177 & 0.0113 & 0.0703 & 1.62 & 0.62 \\
12 & 4.2007 & 0.0118 & -0.0468 & -1.08 & 0.65 \\
13 & 4.2120 & 0.0130 & -0.0355 & -0.82 & 0.72 \\
14 & 4.2000 & 0.0147 & -0.0475 & -1.10 & 0.81 \\
15 & 4.2483 & 0.0194 & 0.0008 & 0.02 & 1.07 \\
16 & 4.2783 & 0.0147 & 0.0308 & 0.71 & 0.81 \\
17 & 4.2062 & 0.0141 & -0.0413 & -0.95 & 0.78 \\
18 & 4.3398 & 0.0431 & 0.0923 & 2.13 & 2.38 \\
19 & 4.2083 & 0.0075 & -0.0392 & -0.90 & 0.42 \\
20 & 4.2683 & 0.0041 & 0.0208 & 0.48 & 0.23 \\
21 & 4.2663 & 0.0042 & 0.0189 & 0.44 & 0.23 \\
22 & 4.2150 & 0.0055 & -0.0325 & -0.75 & 0.30 \\
23 & 4.2067 & 0.0082 & -0.0408 & -0.94 & 0.45 \\
24 & 4.2167 & 0.0082 & -0.0308 & -0.71 & 0.45 \\
\hline & & & & &
\end{tabular}

Average of cell averages $\quad=\quad 4.24748$

Standard Deviation of cell averages $=0.04331$

Repeatability Standard Deviation $\quad=0.01812$

Reproducibility Standard Deviation $=0.04636$

$\mathrm{h}, \mathrm{k}$ Critical Values $\quad=\quad 1.79000,2.59000$ 
Table 5 Precision Statistics $\mathrm{Fe}_{2} \mathrm{O}_{3}$ by XRF glass and powder preparations for replicates 1 and 2

$\mathrm{Fe}_{2} \mathrm{O}_{3}$ Glass, Replicate 1

\begin{tabular}{lllllll}
\hline Material & Xbar & $\boldsymbol{s}_{\boldsymbol{x}}$ & $\boldsymbol{s}_{\boldsymbol{r}}$ & $\boldsymbol{s}_{\boldsymbol{R}}$ & $\boldsymbol{r}$ & $\boldsymbol{R}$ \\
\hline 1 & 2.7363 & 0.0283 & 0.0138 & 0.0310 & 0.04 & 0.09 \\
2 & 4.2401 & 0.0291 & 0.0108 & 0.0308 & 0.03 & 0.09 \\
\hline
\end{tabular}

$\mathrm{Fe}_{2} \mathrm{O}_{3}$ Glass, Replicate 2

\begin{tabular}{lllllll}
\hline Material & Xbar & $\boldsymbol{s}_{\boldsymbol{x}}$ & $\boldsymbol{s}_{\boldsymbol{r}}$ & $\boldsymbol{s}_{\boldsymbol{R}}$ & $\boldsymbol{r}$ & $\boldsymbol{R}$ \\
\hline 1 & 2.7371 & 0.0287 & 0.0109 & 0.0303 & 0.03 & 0.08 \\
2 & 4.2399 & 0.0309 & 0.0173 & 0.0347 & 0.05 & 0.10 \\
\hline
\end{tabular}

$\mathrm{Fe}_{2} \mathrm{O}_{3}$ Powder, Replicate 1

\begin{tabular}{lllllll}
\hline Material & Xbar & $\boldsymbol{s}_{\boldsymbol{x}}$ & $\boldsymbol{s}_{\boldsymbol{r}}$ & $\boldsymbol{s}_{\boldsymbol{R}}$ & $\boldsymbol{r}$ & $\boldsymbol{R}$ \\
\hline 1 & 2.7444 & 0.0440 & 0.0127 & 0.0455 & 0.04 & 0.13 \\
2 & 4.2488 & 0.0422 & 0.0187 & 0.0456 & 0.05 & 0.13 \\
\hline
\end{tabular}

$\mathrm{Fe}_{2} \mathrm{O}_{3}$ Powder, Replicate 2

\begin{tabular}{lllllll}
\hline Material & Xbar & $\boldsymbol{s}_{\boldsymbol{x}}$ & $\boldsymbol{s}_{\boldsymbol{r}}$ & $\boldsymbol{s}_{\boldsymbol{R}}$ & $\boldsymbol{r}$ & $\boldsymbol{R}$ \\
\hline 1 & 2.7442 & 0.0448 & 0.0140 & 0.0466 & 0.04 & 0.13 \\
2 & 4.2475 & 0.0433 & 0.0181 & 0.0464 & 0.05 & 0.13 \\
\hline
\end{tabular}

\section{Fe2O3}

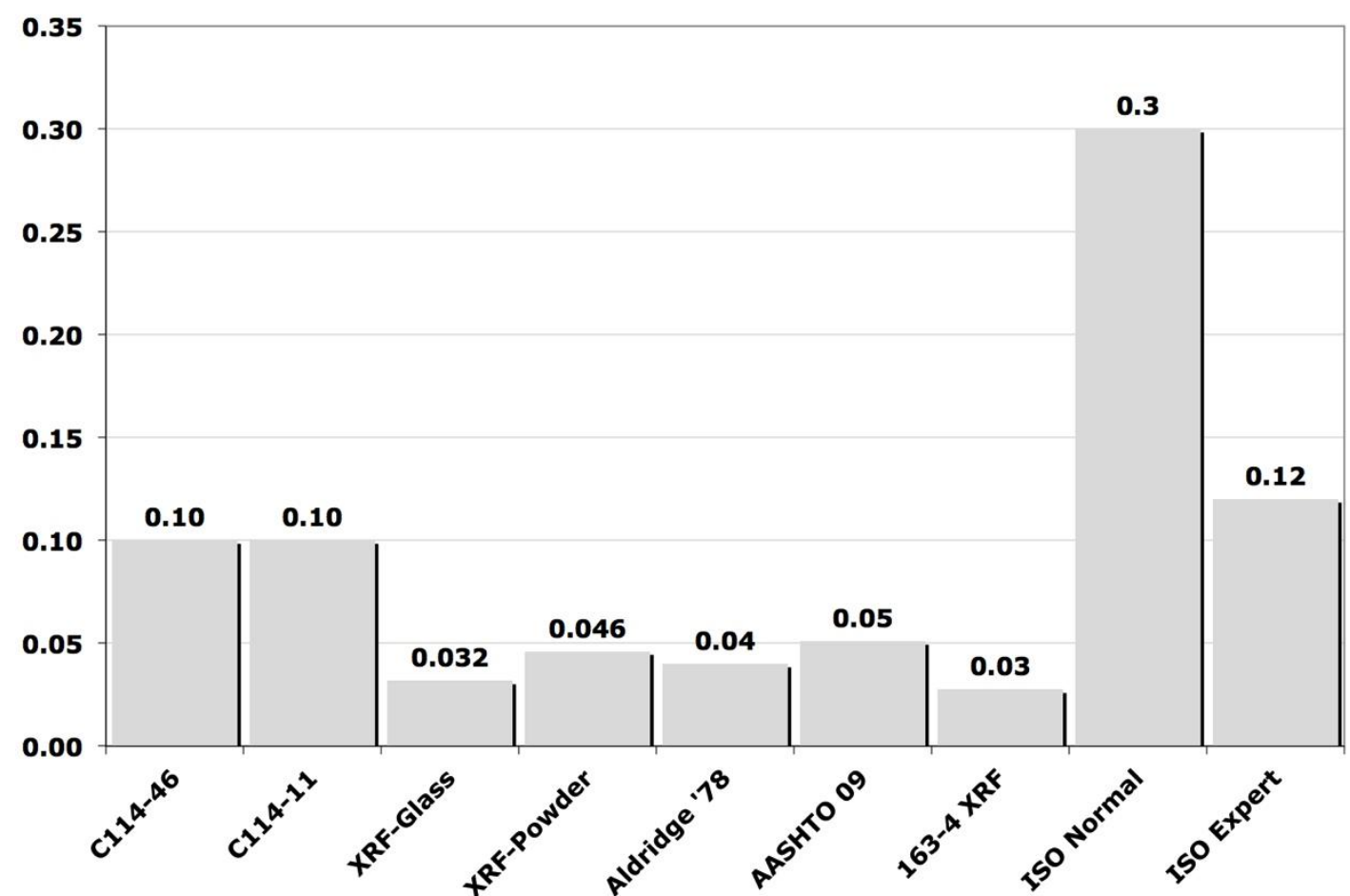

Figure $12 \mathrm{Fe}_{2} \mathrm{O}_{3}$ precision statistics by method with bar chart comparing results to current and past ASTM C114 limits and previous studies on chemical analysis precision as 1-sigma, between lab $\left(S_{R}\right)$. 
$\mathrm{CaO}$

$\mathrm{CaO}$
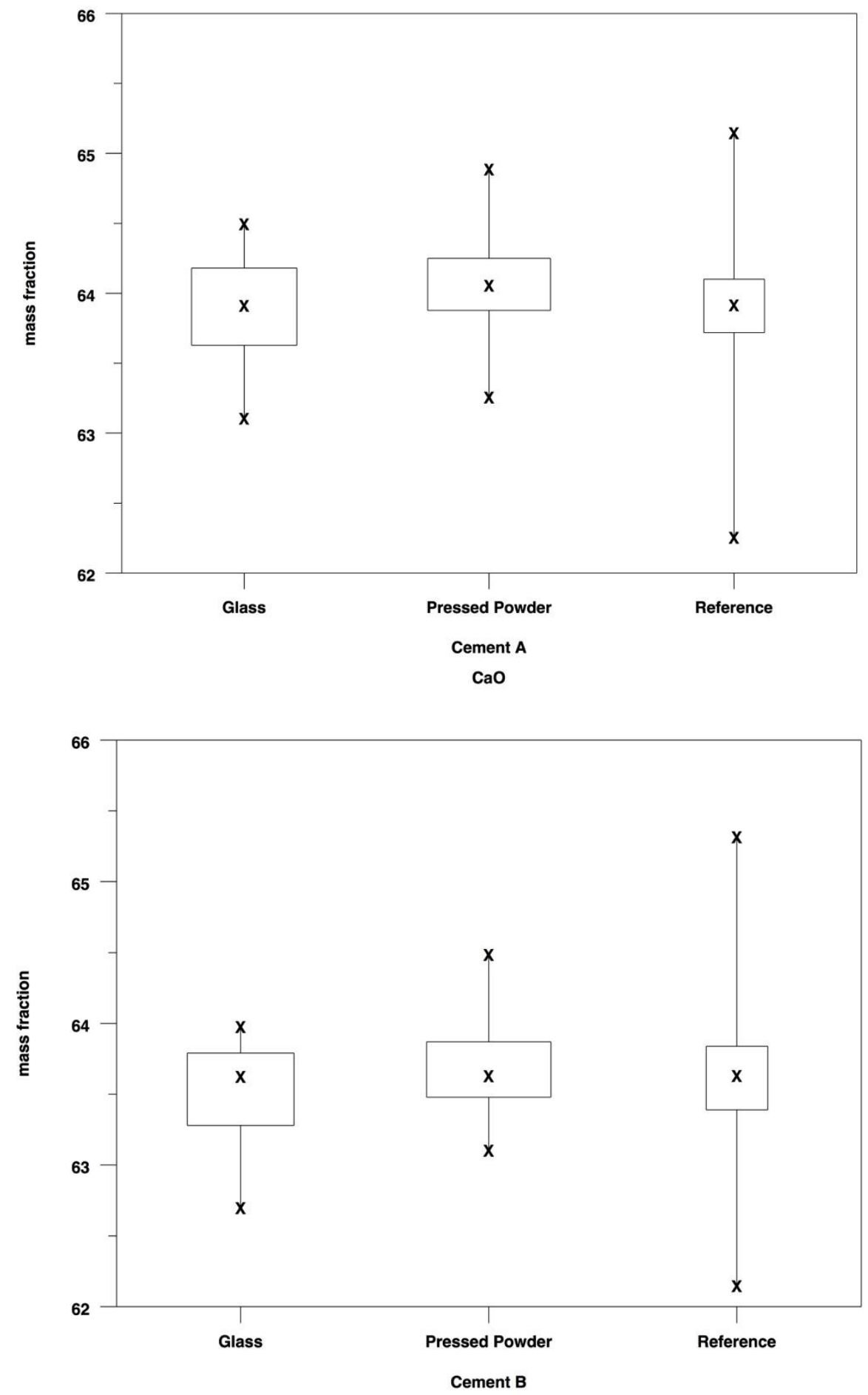

Figure 13 Box plots for CaO XRF glass and powder, and reference methods. 
CaO XRF, Cements A B, Glass, Replicate 1, Material: 1

\begin{tabular}{crrrrr}
\hline $\begin{array}{c}\text { Laboratory } \\
\text { Number }\end{array}$ & $\begin{array}{r}\text { Cell } \\
\text { Mean }\end{array}$ & $\begin{array}{r}\text { Cell } \\
\text { SD }\end{array}$ & $\boldsymbol{d}$ & $\boldsymbol{h}$ & $\boldsymbol{k}$ \\
\hline 1 & 64.1400 & 0.0636 & 0.2613 & 0.75 & 0.60 \\
2 & 64.1667 & 0.1593 & 0.2880 & 0.82 & 1.50 \\
3 & 63.8800 & 0.1188 & 0.0013 & 0.00 & 1.12 \\
4 & 63.2650 & 0.1199 & -0.6137 & -1.75 & 1.13 \\
5 & 63.6978 & 0.0798 & -0.1808 & -0.52 & 0.75 \\
6 & 64.0700 & 0.1664 & 0.1913 & 0.55 & 1.57 \\
7 & 63.6200 & 0.0856 & -0.2587 & -0.74 & 0.81 \\
8 & 64.3000 & 0.0465 & 0.4213 & 1.20 & 0.44 \\
9 & 63.6295 & 0.1095 & -0.2492 & -0.71 & 1.03 \\
10 & 63.9867 & 0.0520 & 0.1080 & 0.31 & 0.49 \\
11 & 63.3730 & 0.1117 & -0.5057 & -1.45 & 1.05 \\
12 & 64.1167 & 0.1340 & 0.2380 & 0.68 & 1.26 \\
13 & 63.8780 & 0.0258 & -0.0007 & 0.00 & 0.24 \\
14 & 64.2683 & 0.0889 & 0.3897 & 1.11 & 0.84 \\
15 & 63.2773 & 0.1387 & -0.6013 & -1.72 & 1.31 \\
16 & 64.3417 & 0.1221 & 0.4630 & 1.32 & 1.15 \\
17 & 63.9267 & 0.0455 & 0.0480 & 0.14 & 0.43 \\
\hline
\end{tabular}

Average of cell averages $\quad=\quad 63.87867$

Standard Deviation of cell averages $=0.34980$

Repeatability Standard Deviation $=0.10610$

Reproducibility Standard Deviation $=0.36296$

$\mathrm{h}, \mathrm{k}$ Critical Values $\quad=\quad 2.51000,1.78000$ 
CaO XRF, Cements A B, Glass, Replicate 1, Material: 2

\begin{tabular}{crrrrr}
\hline $\begin{array}{c}\text { Laboratory } \\
\text { Number }\end{array}$ & $\begin{array}{r}\text { Cell } \\
\text { Mean }\end{array}$ & $\begin{array}{r}\text { Cell } \\
\text { SD }\end{array}$ & $\boldsymbol{d}$ & $\boldsymbol{h}$ & $\boldsymbol{k}$ \\
\hline 1 & 63.7133 & 0.1498 & 0.1971 & 0.58 & 1.19 \\
2 & 63.7000 & 0.2320 & 0.1838 & 0.54 & 1.84 \\
3 & 63.6033 & 0.1350 & 0.0871 & 0.26 & 1.07 \\
4 & 62.8905 & 0.1192 & -0.6257 & -1.85 & 0.95 \\
5 & 63.4462 & 0.1039 & -0.0701 & -0.21 & 0.82 \\
6 & 63.7450 & 0.1787 & 0.2288 & 0.68 & 1.42 \\
7 & 63.0617 & 0.0454 & -0.4546 & -1.34 & 0.36 \\
8 & 63.8283 & 0.0407 & 0.3121 & 0.92 & 0.32 \\
9 & 62.8667 & 0.1388 & -0.6496 & -1.92 & 1.10 \\
10 & 63.8050 & 0.0848 & 0.2888 & 0.85 & 0.67 \\
11 & 63.0392 & 0.0276 & -0.4771 & -1.41 & 0.22 \\
12 & 63.7183 & 0.0567 & 0.2021 & 0.60 & 0.45 \\
13 & 63.6280 & 0.0458 & 0.1118 & 0.33 & 0.36 \\
14 & 63.7733 & 0.0857 & 0.2571 & 0.76 & 0.68 \\
15 & 63.4070 & 0.2229 & -0.1092 & -0.32 & 1.77 \\
16 & 63.7583 & 0.1560 & 0.2421 & 0.72 & 1.24 \\
17 & 63.7917 & 0.0355 & 0.2754 & 0.81 & 0.28 \\
\hline
\end{tabular}

Average of cell averages $\quad=\quad 63.51622$

Standard Deviation of cell averages $=0.33836$

Repeatability Standard Deviation $=0.12610$

Reproducibility Standard Deviation $=0.35740$

$\mathrm{h}, \mathrm{k}$ Critical Values $\quad=\quad 2.51000,1.78000$ 
CaO XRF, Cements A B, Glass, Replicate 2, Material: 1

\begin{tabular}{crrrrr}
\hline $\begin{array}{c}\text { Laboratory } \\
\text { Number }\end{array}$ & $\begin{array}{r}\text { Cell } \\
\text { Mean }\end{array}$ & $\begin{array}{r}\text { Cell } \\
\text { SD }\end{array}$ & $\boldsymbol{d}$ & $\boldsymbol{h}$ & $\boldsymbol{k}$ \\
\hline 1 & 64.1583 & 0.1206 & 0.2863 & 0.79 & 1.14 \\
2 & 64.1617 & 0.1412 & 0.2897 & 0.80 & 1.34 \\
3 & 63.7717 & 0.0962 & -0.1003 & -0.28 & 0.91 \\
4 & 63.2313 & 0.0610 & -0.6407 & -1.77 & 0.58 \\
5 & 63.6963 & 0.0448 & -0.1757 & -0.48 & 0.42 \\
6 & 64.1283 & 0.1876 & 0.2563 & 0.71 & 1.78 \\
7 & 63.6500 & 0.0600 & -0.2220 & -0.61 & 0.57 \\
8 & 64.3050 & 0.0532 & 0.4330 & 1.19 & 0.50 \\
9 & 63.5303 & 0.0934 & -0.3417 & -0.94 & 0.88 \\
10 & 63.9833 & 0.1065 & 0.1113 & 0.31 & 1.01 \\
11 & 63.3885 & 0.0757 & -0.4835 & -1.33 & 0.72 \\
12 & 64.1483 & 0.1347 & 0.2763 & 0.76 & 1.28 \\
13 & 63.8647 & 0.0214 & -0.0073 & -0.02 & 0.20 \\
14 & 64.2767 & 0.0301 & 0.4047 & 1.12 & 0.29 \\
15 & 63.2727 & 0.1657 & -0.5993 & -1.65 & 1.57 \\
16 & 64.3433 & 0.1421 & 0.4713 & 1.30 & 1.35 \\
17 & 63.9133 & 0.0742 & 0.0413 & 0.11 & 0.70 \\
\hline
\end{tabular}

Average of cell averages $\quad=\quad 63.87199$

Standard Deviation of cell averages $=0.36279$

Repeatability Standard Deviation $=0.10558$

Reproducibility Standard Deviation $=0.37538$

$\mathrm{h}, \mathrm{k}$ Critical Values $\quad=\quad 2.51000,1.78000$ 
CaO XRF, Cements A B, Glass, Replicate 2, Material: 2

\begin{tabular}{crrrrr}
\hline $\begin{array}{c}\text { Laboratory } \\
\text { Number }\end{array}$ & $\begin{array}{r}\text { Cell } \\
\text { Mean }\end{array}$ & $\begin{array}{r}\text { Cell } \\
\text { SD }\end{array}$ & $\boldsymbol{d}$ & $\boldsymbol{h}$ & $\boldsymbol{k}$ \\
\hline 1 & 63.6733 & 0.1934 & 0.1711 & 0.46 & 1.44 \\
2 & 63.6983 & 0.2303 & 0.1961 & 0.53 & 1.71 \\
3 & 63.6117 & 0.1606 & 0.1095 & 0.29 & 1.19 \\
4 & 62.8562 & 0.1561 & -0.6460 & -1.74 & 1.16 \\
5 & 63.4400 & 0.0577 & -0.0622 & -0.17 & 0.43 \\
6 & 63.7383 & 0.1668 & 0.2361 & 0.63 & 1.24 \\
7 & 62.9967 & 0.0356 & -0.5055 & -1.36 & 0.26 \\
8 & 63.8200 & 0.0518 & 0.3178 & 0.85 & 0.39 \\
9 & 62.7257 & 0.0616 & -0.7765 & -2.09 & 0.46 \\
10 & 63.8283 & 0.1333 & 0.3261 & 0.88 & 0.99 \\
11 & 63.0040 & 0.0575 & -0.4982 & -1.34 & 0.43 \\
12 & 63.7067 & 0.0712 & 0.2045 & 0.55 & 0.53 \\
13 & 63.6203 & 0.0333 & 0.1181 & 0.32 & 0.25 \\
14 & 63.8417 & 0.0833 & 0.3395 & 0.91 & 0.62 \\
15 & 63.4047 & 0.2306 & -0.0975 & -0.26 & 1.72 \\
16 & 63.7633 & 0.1936 & 0.2611 & 0.70 & 1.44 \\
17 & 63.8083 & 0.0534 & 0.3061 & 0.82 & 0.40 \\
\hline
\end{tabular}

Average of cell averages $\quad=\quad 63.50220$

Standard Deviation of cell averages $=0.37193$

Repeatability Standard Deviation $=0.13443$

Reproducibility Standard Deviation $=0.39165$

$\mathrm{h}, \mathrm{k}$ Critical Values $\quad=\quad 2.51000,1.78000$ 
CaO XRF, Cements A B, Powder, Replicate 1, Material: 1

\begin{tabular}{crrrrr}
\hline $\begin{array}{c}\text { Laboratory } \\
\text { Number }\end{array}$ & $\begin{array}{r}\text { Cell } \\
\text { Mean }\end{array}$ & $\begin{array}{r}\text { Cell } \\
\text { SD }\end{array}$ & $\boldsymbol{d}$ & $\boldsymbol{h}$ & $\boldsymbol{k}$ \\
\hline 1 & 63.9297 & 0.1025 & -0.1635 & -0.45 & 0.85 \\
2 & 63.5705 & 0.1548 & -0.5226 & -1.45 & 1.28 \\
3 & 64.1467 & 0.1334 & 0.0536 & 0.15 & 1.10 \\
4 & 64.0683 & 0.2013 & -0.0248 & -0.07 & 1.67 \\
5 & 63.8833 & 0.0476 & -0.2098 & -0.58 & 0.39 \\
6 & 64.2200 & 0.0759 & 0.1269 & 0.35 & 0.63 \\
7 & 63.7500 & 0.1278 & -0.3431 & -0.95 & 1.06 \\
8 & 64.0300 & 0.1401 & -0.0631 & -0.18 & 1.16 \\
9 & 64.0450 & 0.0914 & -0.0481 & -0.13 & 0.76 \\
10 & 63.8583 & 0.1395 & -0.2348 & -0.65 & 1.16 \\
11 & 64.7017 & 0.0567 & 0.6086 & 1.69 & 0.47 \\
12 & 64.7167 & 0.1538 & 0.6236 & 1.73 & 1.27 \\
13 & 64.0217 & 0.0866 & -0.0714 & -0.20 & 0.72 \\
14 & 63.5695 & 0.1675 & -0.5236 & -1.45 & 1.39 \\
15 & 64.2267 & 0.0850 & 0.1336 & 0.37 & 0.70 \\
16 & 64.0937 & 0.1335 & 0.0005 & 0.00 & 1.11 \\
17 & 64.1800 & 0.0400 & 0.0869 & 0.24 & 0.33 \\
18 & 63.4662 & 0.1279 & -0.6269 & -1.74 & 1.06 \\
19 & 63.9800 & 0.1073 & -0.1131 & -0.31 & 0.89 \\
20 & 64.7093 & 0.1647 & 0.6162 & 1.71 & 1.36 \\
21 & 64.7350 & 0.1178 & 0.6419 & 1.78 & 0.98 \\
22 & 64.2778 & 0.0818 & 0.1847 & 0.51 & 0.68 \\
23 & 63.9617 & 0.0773 & -0.1315 & -0.36 & 0.64 \\
\hline & & & & &
\end{tabular}

Average of cell averages $\quad=64.09312$

Standard Deviation of cell averages $=0.36027$

Repeatability Standard Deviation $=0.12072$

Reproducibility Standard Deviation $=0.37674$

$\mathrm{h}, \mathrm{k}$ Critical Values $\quad=\quad 2.59000,1.79000$ 
CaO XRF, Cements A B, Powder, Replicate 1, Material: 2

\begin{tabular}{crrrrr}
\hline $\begin{array}{c}\text { Laboratory } \\
\text { Number }\end{array}$ & $\begin{array}{r}\text { Cell } \\
\text { Mean }\end{array}$ & $\begin{array}{r}\text { Cell } \\
\text { SD }\end{array}$ & $\boldsymbol{d}$ & $\boldsymbol{h}$ & $\boldsymbol{k}$ \\
\hline 1 & 63.5562 & 0.1137 & -0.1417 & -0.50 & 1.11 \\
2 & 63.4558 & 0.1165 & -0.2420 & -0.86 & 1.14 \\
3 & 63.7900 & 0.0925 & 0.0921 & 0.33 & 0.90 \\
4 & 63.6467 & 0.0703 & -0.0512 & -0.18 & 0.69 \\
5 & 64.0333 & 0.0850 & 0.3355 & 1.19 & 0.83 \\
6 & 63.7083 & 0.0591 & 0.0105 & 0.04 & 0.58 \\
7 & 63.2683 & 0.0900 & -0.4295 & -1.53 & 0.88 \\
8 & 63.5617 & 0.1134 & -0.1362 & -0.48 & 1.11 \\
9 & 63.7367 & 0.0665 & 0.0388 & 0.14 & 0.65 \\
10 & 63.5983 & 0.0279 & -0.0995 & -0.35 & 0.27 \\
11 & 64.1850 & 0.0997 & 0.4871 & 1.73 & 0.97 \\
12 & 64.1817 & 0.1025 & 0.4838 & 1.72 & 1.00 \\
13 & 63.4417 & 0.0479 & -0.2562 & -0.91 & 0.47 \\
14 & 63.3940 & 0.2189 & -0.3039 & -1.08 & 2.14 \\
15 & 63.7867 & 0.0561 & 0.0888 & 0.32 & 0.55 \\
16 & 63.5303 & 0.0568 & -0.1675 & -0.60 & 0.55 \\
17 & 63.5967 & 0.0850 & -0.1012 & -0.36 & 0.83 \\
18 & 63.4042 & 0.1049 & -0.2937 & -1.05 & 1.02 \\
19 & 63.4367 & 0.1037 & -0.2612 & -0.93 & 1.01 \\
20 & 64.0197 & 0.1144 & 0.3218 & 1.15 & 1.12 \\
21 & 64.2733 & 0.1363 & 0.5755 & 2.05 & 1.33 \\
22 & 63.8872 & 0.1513 & 0.1893 & 0.67 & 1.48 \\
23 & 63.5583 & 0.0659 & -0.1395 & -0.50 & 0.64 \\
\hline & & & & &
\end{tabular}

Average of cell averages $\quad=\quad 63.69785$

Standard Deviation of cell averages $=0.28098$

Repeatability Standard Deviation $=0.10254$

Reproducibility Standard Deviation $=0.29616$

$\mathrm{h}, \mathrm{k}$ Critical Values $\quad=\quad 2.59000,1.79000$ 
CaO XRF, Cements A B, Powder, Replicate 2, Material: 1

\begin{tabular}{crrrrr}
\hline $\begin{array}{c}\text { Laboratory } \\
\text { Number }\end{array}$ & $\begin{array}{r}\text { Cell } \\
\text { Mean }\end{array}$ & $\begin{array}{r}\text { Cell } \\
\text { SD }\end{array}$ & $\boldsymbol{d}$ & $\boldsymbol{h}$ & $\boldsymbol{k}$ \\
\hline 1 & 63.9270 & 0.1337 & -0.1910 & -0.53 & 1.18 \\
2 & 63.5670 & 0.1663 & -0.5510 & -1.52 & 1.47 \\
3 & 64.2083 & 0.1005 & 0.0904 & 0.25 & 0.89 \\
4 & 64.1217 & 0.1155 & 0.0037 & 0.01 & 1.02 \\
5 & 63.9333 & 0.0579 & -0.1846 & -0.51 & 0.51 \\
6 & 64.3000 & 0.0651 & 0.1820 & 0.50 & 0.57 \\
7 & 63.8967 & 0.1162 & -0.2213 & -0.61 & 1.03 \\
8 & 64.1133 & 0.1134 & -0.0046 & -0.01 & 1.00 \\
9 & 64.0717 & 0.0755 & -0.0463 & -0.13 & 0.67 \\
10 & 63.8350 & 0.1536 & -0.2830 & -0.78 & 1.36 \\
11 & 64.7167 & 0.0524 & 0.5987 & 1.65 & 0.46 \\
12 & 64.7350 & 0.1247 & 0.6170 & 1.71 & 1.10 \\
13 & 64.0350 & 0.1050 & -0.0830 & -0.23 & 0.93 \\
14 & 63.5732 & 0.1811 & -0.5448 & -1.51 & 1.60 \\
15 & 64.2483 & 0.0605 & 0.1304 & 0.36 & 0.53 \\
16 & 64.0127 & 0.0696 & -0.1053 & -0.29 & 0.61 \\
17 & 64.1950 & 0.0288 & 0.0770 & 0.21 & 0.25 \\
18 & 63.4852 & 0.1265 & -0.6328 & -1.75 & 1.12 \\
19 & 64.0150 & 0.1283 & -0.1030 & -0.28 & 1.13 \\
20 & 64.7488 & 0.1806 & 0.6309 & 1.74 & 1.59 \\
21 & 64.7400 & 0.1103 & 0.6220 & 1.72 & 0.97 \\
22 & 64.2910 & 0.0932 & 0.1730 & 0.48 & 0.82 \\
23 & 63.9433 & 0.0753 & -0.1746 & -0.48 & 0.66 \\
\hline & & & & &
\end{tabular}

Average of cell averages $\quad=64.11797$

Standard Deviation of cell averages $=0.36181$

Repeatability Standard Deviation $=0.11330$

Reproducibility Standard Deviation $=0.37630$

$\mathrm{h}, \mathrm{k}$ Critical Values $\quad=\quad 2.59000,1.79000$ 
CaO XRF, Cements A B, Powder, Replicate 2, Material: 2

\begin{tabular}{crrrrr}
\hline $\begin{array}{c}\text { Laboratory } \\
\text { Number }\end{array}$ & $\begin{array}{r}\text { Cell } \\
\text { Mean }\end{array}$ & $\begin{array}{r}\text { Cell } \\
\text { SD }\end{array}$ & $\boldsymbol{d}$ & $\boldsymbol{h}$ & $\boldsymbol{k}$ \\
\hline 1 & 63.6030 & 0.0795 & -0.1167 & -0.42 & 0.83 \\
2 & 63.4658 & 0.1136 & -0.2539 & -0.92 & 1.18 \\
3 & 63.8383 & 0.1034 & 0.1186 & 0.43 & 1.08 \\
4 & 63.6700 & 0.0616 & -0.0497 & -0.18 & 0.64 \\
5 & 64.0017 & 0.0643 & 0.2820 & 1.03 & 0.67 \\
6 & 63.7783 & 0.0608 & 0.0586 & 0.21 & 0.63 \\
7 & 63.3767 & 0.0845 & -0.3430 & -1.25 & 0.88 \\
8 & 63.5850 & 0.0841 & -0.1347 & -0.49 & 0.87 \\
9 & 63.7433 & 0.0927 & 0.0236 & 0.09 & 0.96 \\
10 & 63.6083 & 0.0688 & -0.1114 & -0.41 & 0.72 \\
11 & 64.2083 & 0.0747 & 0.4886 & 1.78 & 0.78 \\
12 & 64.2417 & 0.0838 & 0.5220 & 1.90 & 0.87 \\
13 & 63.4667 & 0.0794 & -0.2530 & -0.92 & 0.83 \\
14 & 63.4353 & 0.2025 & -0.2844 & -1.04 & 2.11 \\
15 & 63.7883 & 0.0402 & 0.0686 & 0.25 & 0.42 \\
16 & 63.4985 & 0.0454 & -0.2212 & -0.81 & 0.47 \\
17 & 63.6167 & 0.0918 & -0.1030 & -0.38 & 0.95 \\
18 & 63.4195 & 0.1002 & -0.3002 & -1.09 & 1.04 \\
19 & 63.4617 & 0.0601 & -0.2580 & -0.94 & 0.63 \\
20 & 64.0592 & 0.0942 & 0.3395 & 1.24 & 0.98 \\
21 & 64.2517 & 0.1339 & 0.5320 & 1.94 & 1.39 \\
22 & 63.8885 & 0.1602 & 0.1688 & 0.61 & 1.67 \\
23 & 63.5467 & 0.0731 & -0.1730 & -0.63 & 0.76 \\
\hline & & & & &
\end{tabular}

Average of cell averages $\quad=63.71970$

Standard Deviation of cell averages $=0.27469$

Repeatability Standard Deviation $=0.09613$

Reproducibility Standard Deviation $=0.28837$

$\mathrm{h}, \mathrm{k}$ Critical Values $\quad=\quad 2.59000,1.79000$ 
Table 6 Precision Statistics $\mathrm{CaO}$ by XRF glass and powder preparations for replicates 1 and 2

\section{CaO Glass, Replicate 1}

\begin{tabular}{crrrrrr}
\hline Material & Xbar & $\boldsymbol{s}_{\boldsymbol{x}}$ & $\boldsymbol{s}_{\boldsymbol{r}}$ & $\boldsymbol{s}_{\boldsymbol{R}}$ & $\boldsymbol{r}$ & $\boldsymbol{R}$ \\
\hline 1 & 63.8787 & 0.3498 & 0.1061 & 0.3630 & 0.30 & 1.02 \\
2 & 63.5162 & 0.3384 & 0.1261 & 0.3574 & 0.35 & 1.00 \\
\hline
\end{tabular}

CaO Glass, Replicate 2

\begin{tabular}{crrrrrr}
\hline Material & Xbar & $\boldsymbol{s}_{\boldsymbol{x}}$ & $\boldsymbol{s}_{\boldsymbol{r}}$ & $\boldsymbol{s}_{\boldsymbol{R}}$ & $\boldsymbol{r}$ & $\boldsymbol{R}$ \\
\hline 1 & 63.8720 & 0.3628 & 0.1056 & 0.3754 & 0.30 & 1.05 \\
2 & 63.5022 & 0.3719 & 0.1344 & 0.3916 & 0.38 & 1.10 \\
\hline
\end{tabular}

\section{CaO Powder, Replicate 1}

\begin{tabular}{crrrrrr}
\hline Material & Xbar & $\boldsymbol{s}_{\boldsymbol{x}}$ & $\boldsymbol{s}_{\boldsymbol{r}}$ & $\boldsymbol{s}_{\boldsymbol{R}}$ & $\boldsymbol{r}$ & $\boldsymbol{R}$ \\
\hline 1 & 64.0931 & 0.3603 & 0.1207 & 0.3767 & 0.34 & 1.05 \\
2 & 63.6979 & 0.2810 & 0.1025 & 0.2962 & 0.29 & 0.83 \\
\hline
\end{tabular}

CaO Powder, Replicate 2

\begin{tabular}{crrrrrr}
\hline Material & Xbar & $\boldsymbol{s}_{\boldsymbol{x}}$ & $\boldsymbol{s}_{\boldsymbol{r}}$ & $\boldsymbol{s}_{\boldsymbol{R}}$ & $\boldsymbol{r}$ & $\boldsymbol{R}$ \\
\hline 1 & 64.1180 & 0.3618 & 0.1133 & 0.3763 & 0.32 & 1.05 \\
2 & 63.7197 & 0.2747 & 0.0961 & 0.2884 & 0.27 & 0.81 \\
\hline
\end{tabular}

CaO

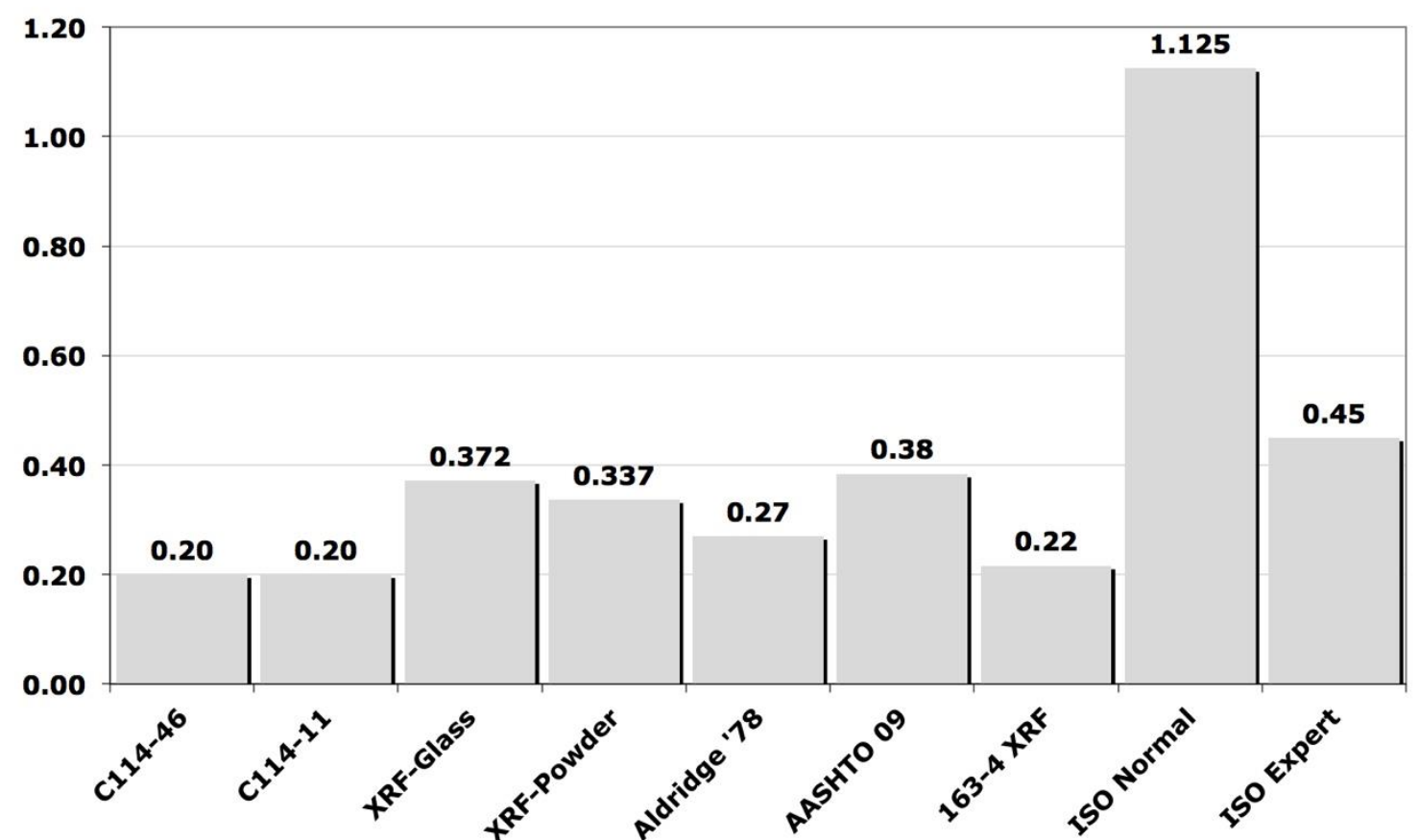

Figure $14 \mathrm{CaO}$ precision statistics by method with bar chart comparing results to current and past ASTM C114 limits and previous studies on chemical analysis precision as 1-sigma, between lab $\left(S_{R}\right)$. 
MgO
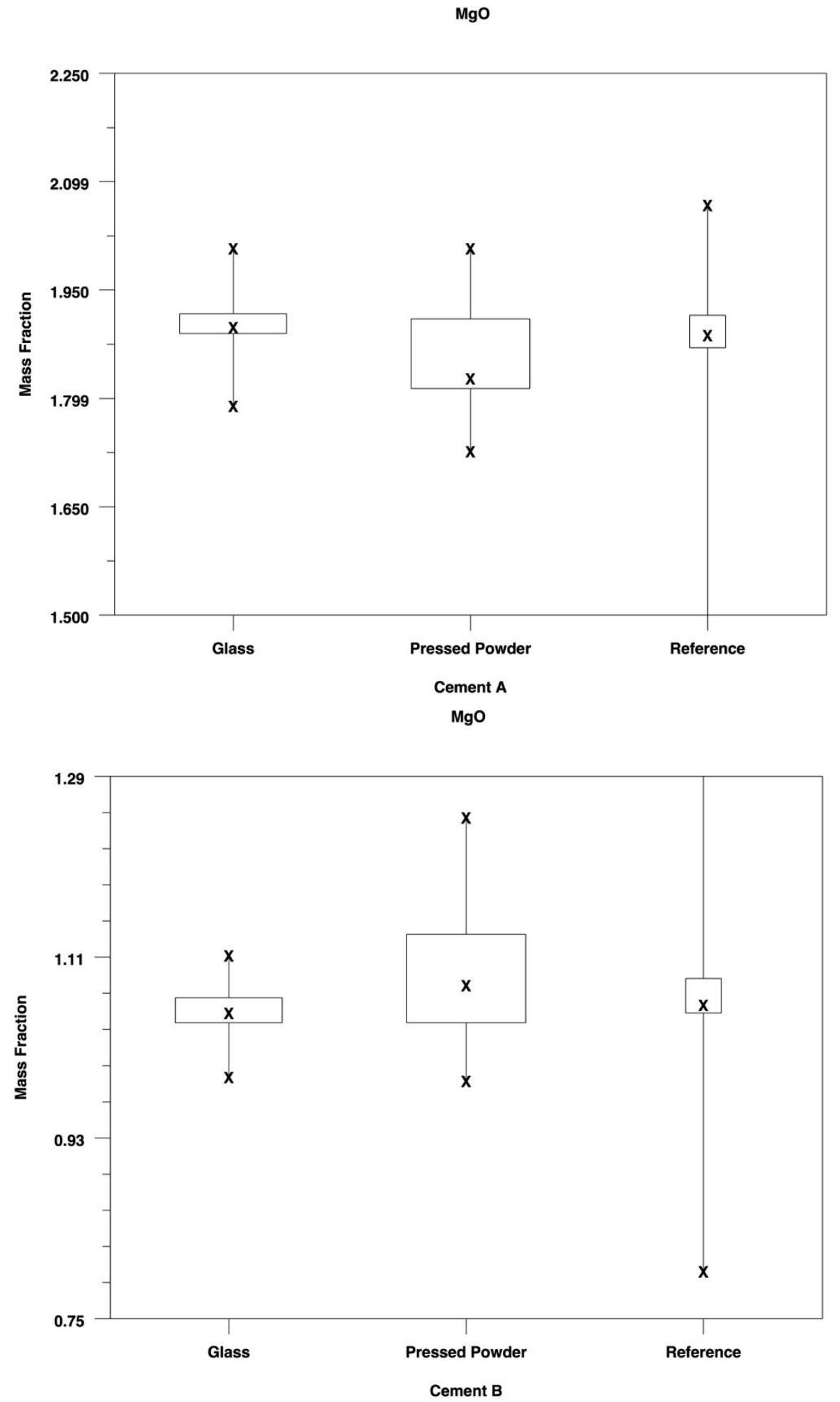

Figure 15 Box plots for MgO XRF glass and powder, and reference methods. 
MgO XRF, Cements A B, Glass, Replicate 1, Material: 1

\begin{tabular}{crrrrr}
\hline $\begin{array}{c}\text { Laboratory } \\
\text { Number }\end{array}$ & $\begin{array}{r}\text { Cell } \\
\text { Mean }\end{array}$ & $\begin{array}{r}\text { Cell } \\
\text { SD }\end{array}$ & $\boldsymbol{d}$ & $\boldsymbol{h}$ & $\boldsymbol{k}$ \\
\hline 1 & 1.8828 & 0.0081 & -0.0225 & -0.81 & 0.56 \\
2 & 1.8715 & 0.0422 & -0.0338 & -1.22 & 2.90 \\
3 & 1.9067 & 0.0046 & 0.0013 & 0.05 & 0.32 \\
4 & 1.8983 & 0.0041 & -0.0070 & -0.25 & 0.28 \\
5 & 1.9037 & 0.0084 & -0.0017 & -0.06 & 0.57 \\
6 & 1.9050 & 0.0084 & -0.0003 & -0.01 & 0.57 \\
7 & 1.9867 & 0.0103 & 0.0813 & 2.93 & 0.71 \\
8 & 1.9320 & 0.0049 & 0.0267 & 0.96 & 0.34 \\
9 & 1.8667 & 0.0163 & -0.0387 & -1.39 & 1.12 \\
10 & 1.8833 & 0.0103 & -0.0220 & -0.79 & 0.71 \\
11 & 1.9217 & 0.0172 & 0.0163 & 0.59 & 1.18 \\
12 & 1.9317 & 0.0117 & 0.0263 & 0.95 & 0.80 \\
13 & 1.8953 & 0.0173 & -0.0100 & -0.36 & 1.19 \\
14 & 1.8983 & 0.0075 & -0.0070 & -0.25 & 0.52 \\
15 & 1.8933 & 0.0137 & -0.0120 & -0.43 & 0.94 \\
16 & 1.9135 & 0.0044 & 0.0081 & 0.29 & 0.30 \\
17 & 1.9000 & 0.0089 & -0.0053 & -0.19 & 0.61 \\
\hline
\end{tabular}

Average of cell averages $\quad=\quad 1.90532$

Standard Deviation of cell averages $=0.02779$

Repeatability Standard Deviation $=0.01457$

Reproducibility Standard Deviation $=0.03081$

$\mathrm{h}, \mathrm{k}$ Critical Values $\quad=\quad 2.51000,1.78000$ 
MgO XRF, Cements A B, Glass, Replicate 1, Material: 2

\begin{tabular}{crrrrr}
\hline $\begin{array}{c}\text { Laboratory } \\
\text { Number }\end{array}$ & $\begin{array}{r}\text { Cell } \\
\text { Mean }\end{array}$ & $\begin{array}{r}\text { Cell } \\
\text { SD }\end{array}$ & $\boldsymbol{d}$ & $\boldsymbol{h}$ & $\boldsymbol{k}$ \\
\hline 1 & 1.0490 & 0.0055 & -0.0163 & -0.75 & 0.57 \\
2 & 1.0372 & 0.0218 & -0.0282 & -1.30 & 2.26 \\
3 & 1.0603 & 0.0048 & -0.0050 & -0.23 & 0.50 \\
4 & 1.0683 & 0.0075 & 0.0030 & 0.14 & 0.78 \\
5 & 1.0662 & 0.0035 & 0.0008 & 0.04 & 0.36 \\
6 & 1.0650 & 0.0055 & -0.0003 & -0.02 & 0.57 \\
7 & 1.1050 & 0.0105 & 0.0397 & 1.83 & 1.09 \\
8 & 1.0785 & 0.0054 & 0.0132 & 0.61 & 0.56 \\
9 & 1.1050 & 0.0105 & 0.0397 & 1.83 & 1.09 \\
10 & 1.0483 & 0.0041 & -0.0170 & -0.78 & 0.42 \\
11 & 1.1017 & 0.0117 & 0.0363 & 1.67 & 1.21 \\
12 & 1.0583 & 0.0117 & -0.0070 & -0.32 & 1.21 \\
13 & 1.0445 & 0.0144 & -0.0208 & -0.96 & 1.50 \\
14 & 1.0417 & 0.0041 & -0.0237 & -1.09 & 0.42 \\
15 & 1.0750 & 0.0084 & 0.0097 & 0.45 & 0.87 \\
16 & 1.0600 & 0.0031 & -0.0053 & -0.25 & 0.32 \\
17 & 1.0467 & 0.0103 & -0.0187 & -0.86 & 1.07 \\
\hline
\end{tabular}

Average of cell averages $\quad=\quad 1.06533$

Standard Deviation of cell averages $=0.02171$

Repeatability Standard Deviation $=0.00962$

Reproducibility Standard Deviation $=0.02342$

$\mathrm{h}, \mathrm{k}$ Critical Values $\quad=\quad 2.51000,1.78000$ 
MgO XRF, Cements A B, Glass, Replicate 2, Material: 1

\begin{tabular}{crrrrr}
\hline $\begin{array}{c}\text { Laboratory } \\
\text { Number }\end{array}$ & $\begin{array}{r}\text { Cell } \\
\text { Mean }\end{array}$ & $\begin{array}{r}\text { Cell } \\
\text { SD }\end{array}$ & $\boldsymbol{d}$ & $\boldsymbol{h}$ & $\boldsymbol{k}$ \\
\hline 1 & 1.8907 & 0.0167 & -0.0164 & -0.60 & 1.10 \\
2 & 1.8750 & 0.0421 & -0.0321 & -1.18 & 2.78 \\
3 & 1.9087 & 0.0029 & 0.0016 & 0.06 & 0.19 \\
4 & 1.8917 & 0.0075 & -0.0154 & -0.57 & 0.50 \\
5 & 1.9037 & 0.0072 & -0.0034 & -0.12 & 0.47 \\
6 & 1.9117 & 0.0117 & 0.0046 & 0.17 & 0.77 \\
7 & 1.9900 & 0.0141 & 0.0829 & 3.04 & 0.93 \\
8 & 1.9298 & 0.0053 & 0.0228 & 0.84 & 0.35 \\
9 & 1.8767 & 0.0163 & -0.0304 & -1.12 & 1.08 \\
10 & 1.8800 & 0.0063 & -0.0271 & -0.99 & 0.42 \\
11 & 1.9167 & 0.0121 & 0.0096 & 0.35 & 0.80 \\
12 & 1.9350 & 0.0055 & 0.0279 & 1.03 & 0.36 \\
13 & 1.9130 & 0.0224 & 0.0059 & 0.22 & 1.48 \\
14 & 1.9000 & 0.0155 & -0.0071 & -0.26 & 1.02 \\
15 & 1.8967 & 0.0082 & -0.0104 & -0.38 & 0.54 \\
16 & 1.9076 & 0.0048 & 0.0005 & 0.02 & 0.32 \\
17 & 1.8933 & 0.0082 & -0.0137 & -0.50 & 0.54 \\
\hline
\end{tabular}

Average of cell averages $\quad=\quad 1.90706$

Standard Deviation of cell averages $=0.02725$

Repeatability Standard Deviation $=0.01517$

Reproducibility Standard Deviation $=0.03056$

$\mathrm{h}, \mathrm{k}$ Critical Values $\quad=\quad 2.51000,1.78000$ 
MgO XRF, Cements A B, Glass, Replicate 2, Material: 2

\begin{tabular}{crrrrr}
\hline $\begin{array}{c}\text { Laboratory } \\
\text { Number }\end{array}$ & $\begin{array}{r}\text { Cell } \\
\text { Mean }\end{array}$ & $\begin{array}{r}\text { Cell } \\
\text { SD }\end{array}$ & $\boldsymbol{d}$ & $\boldsymbol{h}$ & $\boldsymbol{k}$ \\
\hline 1 & 1.0452 & 0.0040 & -0.0196 & -1.22 & 0.46 \\
2 & 1.0435 & 0.0229 & -0.0213 & -1.32 & 2.62 \\
3 & 1.0598 & 0.0050 & -0.0050 & -0.31 & 0.58 \\
4 & 1.0667 & 0.0103 & 0.0019 & 0.12 & 1.19 \\
5 & 1.0648 & 0.0025 & 0.0000 & 0.00 & 0.29 \\
6 & 1.0667 & 0.0052 & 0.0019 & 0.12 & 0.59 \\
7 & 1.0967 & 0.0052 & 0.0319 & 1.98 & 0.59 \\
8 & 1.0738 & 0.0065 & 0.0090 & 0.56 & 0.75 \\
9 & 1.0933 & 0.0103 & 0.0285 & 1.77 & 1.19 \\
10 & 1.0483 & 0.0041 & -0.0165 & -1.02 & 0.47 \\
11 & 1.0850 & 0.0084 & 0.0202 & 1.25 & 0.96 \\
12 & 1.0683 & 0.0075 & 0.0035 & 0.22 & 0.86 \\
13 & 1.0585 & 0.0118 & -0.0063 & -0.39 & 1.36 \\
14 & 1.0500 & 0.0063 & -0.0148 & -0.92 & 0.73 \\
15 & 1.0750 & 0.0055 & 0.0102 & 0.63 & 0.63 \\
16 & 1.0574 & 0.0011 & -0.0074 & -0.46 & 0.13 \\
17 & 1.0483 & 0.0075 & -0.0165 & -1.02 & 0.86 \\
\hline
\end{tabular}

Average of cell averages $\quad=\quad 1.06479$

Standard Deviation of cell averages $=0.01613$

Repeatability Standard Deviation $=0.00871$

Reproducibility Standard Deviation $=0.01798$

$\mathrm{h}, \mathrm{k}$ Critical Values $\quad=\quad 2.51000,1.78000$ 
MgO XRF, Cements A B, Powder, Replicate 1, Material: 1

\begin{tabular}{crrrrr}
\hline $\begin{array}{c}\text { Laboratory } \\
\text { Number }\end{array}$ & $\begin{array}{r}\text { Cell } \\
\text { Mean }\end{array}$ & $\begin{array}{r}\text { Cell } \\
\text { SD }\end{array}$ & $\boldsymbol{d}$ & $\boldsymbol{h}$ & $\boldsymbol{k}$ \\
\hline 1 & 1.9733 & 0.0137 & 0.1191 & 1.82 & 1.14 \\
2 & 1.8267 & 0.0082 & -0.0275 & -0.42 & 0.68 \\
3 & 1.7952 & 0.0147 & -0.0590 & -0.90 & 1.23 \\
4 & 1.7403 & 0.0065 & -0.1139 & -1.74 & 0.54 \\
5 & 1.9083 & 0.0183 & 0.0541 & 0.83 & 1.54 \\
6 & 1.8450 & 0.0138 & -0.0092 & -0.14 & 1.16 \\
7 & 1.8383 & 0.0133 & -0.0159 & -0.24 & 1.11 \\
8 & 1.9317 & 0.0041 & 0.0775 & 1.18 & 0.34 \\
9 & 1.8450 & 0.0084 & -0.0092 & -0.14 & 0.70 \\
10 & 1.9933 & 0.0151 & 0.1391 & 2.12 & 1.26 \\
12 & 1.9183 & 0.0133 & 0.0641 & 0.98 & 1.11 \\
13 & 1.9121 & 0.0041 & 0.0579 & 0.88 & 0.34 \\
14 & 1.8062 & 0.0118 & -0.0480 & -0.73 & 0.99 \\
17 & 1.8163 & 0.0102 & -0.0379 & -0.58 & 0.85 \\
18 & 1.7733 & 0.0207 & -0.0809 & -1.23 & 1.73 \\
19 & 1.8317 & 0.0041 & -0.0225 & -0.34 & 0.34 \\
20 & 1.9050 & 0.0138 & 0.0508 & 0.78 & 1.16 \\
21 & 1.8161 & 0.0093 & -0.0381 & -0.58 & 0.78 \\
23 & 1.8183 & 0.0098 & -0.0359 & -0.55 & 0.82 \\
25 & 1.8235 & 0.0072 & -0.0307 & -0.47 & 0.60 \\
26 & 1.8200 & 0.0126 & -0.0342 & -0.52 & 1.06 \\
\hline
\end{tabular}

Average of cell averages $=1.85419$

Standard Deviation of cell averages $=0.06550$

Repeatability Standard Deviation $=0.01193$

Reproducibility Standard Deviation $=0.06640$

$\mathrm{h}, \mathrm{k}$ Critical Values $\quad=\quad 2.57000,1.79000$ 
MgO XRF, Cements A B, Powder, Replicate 1, Material: 2

\begin{tabular}{crrrrr}
\hline $\begin{array}{c}\text { Laboratory } \\
\text { Number }\end{array}$ & $\begin{array}{r}\text { Cell } \\
\text { Mean }\end{array}$ & $\begin{array}{r}\text { Cell } \\
\text { SD }\end{array}$ & $\boldsymbol{d}$ & $\boldsymbol{h}$ & $\boldsymbol{k}$ \\
\hline 1 & 1.1483 & 0.0147 & 0.0467 & 0.75 & 1.36 \\
2 & 1.1200 & 0.0110 & 0.0184 & 0.29 & 1.01 \\
3 & 1.1423 & 0.0105 & 0.0407 & 0.65 & 0.97 \\
4 & 1.0102 & 0.0204 & -0.0915 & -1.46 & 1.89 \\
5 & 1.0833 & 0.0121 & -0.0183 & -0.29 & 1.12 \\
6 & 1.0900 & 0.0089 & -0.0116 & -0.19 & 0.83 \\
7 & 1.0600 & 0.0110 & -0.0416 & -0.66 & 1.01 \\
8 & 1.1300 & 0.0089 & 0.0284 & 0.45 & 0.83 \\
9 & 1.1050 & 0.0055 & 0.0034 & 0.05 & 0.51 \\
10 & 1.2583 & 0.0041 & 0.1567 & 2.50 & 0.38 \\
12 & 1.1867 & 0.0137 & 0.0850 & 1.36 & 1.26 \\
13 & 1.1969 & 0.0065 & 0.0952 & 1.52 & 0.60 \\
14 & 1.1198 & 0.0124 & 0.0182 & 0.29 & 1.15 \\
17 & 1.0717 & 0.0049 & -0.0300 & -0.48 & 0.45 \\
18 & 1.0500 & 0.0179 & -0.0516 & -0.82 & 1.65 \\
19 & 1.0583 & 0.0075 & -0.0433 & -0.69 & 0.70 \\
20 & 1.1383 & 0.0117 & 0.0367 & 0.59 & 1.08 \\
21 & 1.0504 & 0.0093 & -0.0513 & -0.82 & 0.86 \\
23 & 1.0417 & 0.0075 & -0.0600 & -0.96 & 0.70 \\
25 & 1.0245 & 0.0019 & -0.0771 & -1.23 & 0.18 \\
26 & 1.0483 & 0.0075 & -0.0533 & -0.85 & 0.70 \\
\hline
\end{tabular}

Average of cell averages $\quad=\quad 1.10162$

Standard Deviation of cell averages $=0.06267$

Repeatability Standard Deviation $=0.01082$

Reproducibility Standard Deviation $=0.06344$

$\mathrm{h}, \mathrm{k}$ Critical Values $\quad=\quad 2.57000,1.79000$ 
MgO XRF, Cements A B, Powder, Replicate 2, Material: 1

\begin{tabular}{crrrrr}
\hline $\begin{array}{c}\text { Laboratory } \\
\text { Number }\end{array}$ & $\begin{array}{r}\text { Cell } \\
\text { Mean }\end{array}$ & $\begin{array}{r}\text { Cell } \\
\text { SD }\end{array}$ & $\boldsymbol{d}$ & $\boldsymbol{h}$ & $\boldsymbol{k}$ \\
\hline 1 & 1.9717 & 0.0299 & 0.1176 & 1.77 & 2.33 \\
2 & 1.8267 & 0.0103 & -0.0274 & -0.41 & 0.80 \\
3 & 1.7955 & 0.0124 & -0.0586 & -0.88 & 0.97 \\
4 & 1.7405 & 0.0071 & -0.1136 & -1.71 & 0.55 \\
5 & 1.9133 & 0.0137 & 0.0592 & 0.89 & 1.06 \\
6 & 1.8433 & 0.0052 & -0.0108 & -0.16 & 0.40 \\
7 & 1.8417 & 0.0194 & -0.0124 & -0.19 & 1.51 \\
8 & 1.9300 & 0.0089 & 0.0759 & 1.15 & 0.70 \\
9 & 1.8467 & 0.0082 & -0.0074 & -0.11 & 0.64 \\
10 & 1.9933 & 0.0151 & 0.1392 & 2.10 & 1.17 \\
12 & 1.9233 & 0.0052 & 0.0692 & 1.04 & 0.40 \\
13 & 1.9139 & 0.0046 & 0.0598 & 0.90 & 0.36 \\
14 & 1.7990 & 0.0170 & -0.0551 & -0.83 & 1.32 \\
17 & 1.8163 & 0.0116 & -0.0378 & -0.57 & 0.90 \\
18 & 1.7667 & 0.0197 & -0.0874 & -1.32 & 1.53 \\
19 & 1.8333 & 0.0052 & -0.0208 & -0.31 & 0.40 \\
20 & 1.9017 & 0.0117 & 0.0476 & 0.72 & 0.91 \\
21 & 1.8145 & 0.0087 & -0.0397 & -0.60 & 0.67 \\
23 & 1.8233 & 0.0121 & -0.0308 & -0.46 & 0.94 \\
25 & 1.8184 & 0.0051 & -0.0357 & -0.54 & 0.40 \\
26 & 1.8233 & 0.0052 & -0.0308 & -0.46 & 0.40 \\
\hline
\end{tabular}

Average of cell averages $\quad=\quad 1.85412$

Standard Deviation of cell averages $=0.06626$

Repeatability Standard Deviation $=0.01285$

Reproducibility Standard Deviation $=0.06729$

$\mathrm{h}, \mathrm{k}$ Critical Values $\quad=\quad 2.57000,1.79000$ 
MgO XRF, Cements A B, Powder, Replicate 2, Material: 2

\begin{tabular}{crrrrr}
\hline $\begin{array}{c}\text { Laboratory } \\
\text { Number }\end{array}$ & $\begin{array}{r}\text { Cell } \\
\text { Mean }\end{array}$ & $\begin{array}{r}\text { Cell } \\
\text { SD }\end{array}$ & $\boldsymbol{d}$ & $\boldsymbol{h}$ & $\boldsymbol{k}$ \\
\hline 1 & 1.1333 & 0.0151 & 0.0334 & 0.53 & 1.42 \\
2 & 1.1317 & 0.0098 & 0.0317 & 0.51 & 0.92 \\
3 & 1.1433 & 0.0069 & 0.0434 & 0.69 & 0.65 \\
4 & 1.0103 & 0.0200 & -0.0896 & -1.43 & 1.88 \\
5 & 1.0700 & 0.0210 & -0.0299 & -0.48 & 1.97 \\
6 & 1.0900 & 0.0063 & -0.0099 & -0.16 & 0.59 \\
7 & 1.0550 & 0.0122 & -0.0449 & -0.72 & 1.15 \\
8 & 1.1317 & 0.0075 & 0.0317 & 0.51 & 0.71 \\
9 & 1.1050 & 0.0055 & 0.0051 & 0.08 & 0.52 \\
10 & 1.2533 & 0.0052 & 0.1534 & 2.45 & 0.49 \\
12 & 1.1883 & 0.0147 & 0.0884 & 1.41 & 1.38 \\
13 & 1.1979 & 0.0032 & 0.0980 & 1.57 & 0.30 \\
14 & 1.1070 & 0.0061 & 0.0071 & 0.11 & 0.58 \\
17 & 1.0725 & 0.0034 & -0.0274 & -0.44 & 0.32 \\
18 & 1.0367 & 0.0121 & -0.0633 & -1.01 & 1.14 \\
19 & 1.0617 & 0.0075 & -0.0383 & -0.61 & 0.71 \\
20 & 1.1383 & 0.0147 & 0.0384 & 0.61 & 1.38 \\
21 & 1.0493 & 0.0085 & -0.0506 & -0.81 & 0.80 \\
23 & 1.0467 & 0.0052 & -0.0533 & -0.85 & 0.49 \\
25 & 1.0246 & 0.0029 & -0.0753 & -1.20 & 0.27 \\
26 & 1.0517 & 0.0075 & -0.0483 & -0.77 & 0.71 \\
\hline
\end{tabular}

Average of cell averages $\quad=\quad 1.09992$

Standard Deviation of cell averages $=0.06252$

Repeatability Standard Deviation $=0.01063$

Reproducibility Standard Deviation $=0.06327$

$\mathrm{h}, \mathrm{k}$ Critical Values $\quad=\quad 2.57000,1.79000$ 
Table 7 Precision Statistics $\mathrm{CaO}$ by XRF glass and powder preparations for replicates 1 and 2

MgO, Glass, Replicate 1

\begin{tabular}{crrrrrr}
\hline Material & Xbar & $\boldsymbol{s}_{\boldsymbol{x}}$ & $\boldsymbol{s}_{\boldsymbol{r}}$ & $\boldsymbol{s}_{\boldsymbol{R}}$ & $\boldsymbol{r}$ & $\boldsymbol{R}$ \\
\hline 1 & 1.9053 & 0.0278 & 0.0146 & 0.0308 & 0.04 & 0.09 \\
2 & 1.0653 & 0.0217 & 0.0096 & 0.0234 & 0.03 & 0.07 \\
\hline
\end{tabular}

MgO, Glass, Replicate 2

\begin{tabular}{crrrrrr}
\hline Material & Xbar & $\boldsymbol{s}_{\boldsymbol{x}}$ & $\boldsymbol{s}_{\boldsymbol{r}}$ & $\boldsymbol{s}_{\boldsymbol{R}}$ & $\boldsymbol{r}$ & $\boldsymbol{R}$ \\
\hline 1 & 1.9071 & 0.0272 & 0.0152 & 0.0306 & 0.04 & 0.09 \\
2 & 1.0648 & 0.0161 & 0.0087 & 0.0180 & 0.02 & 0.05 \\
\hline
\end{tabular}

MgO, Powder, Replicate 1

\begin{tabular}{crrrrrr}
\hline Material & Xbar & $\boldsymbol{s}_{\boldsymbol{x}}$ & $\boldsymbol{s}_{\boldsymbol{r}}$ & $\boldsymbol{s}_{\boldsymbol{R}}$ & $\boldsymbol{r}$ & $\boldsymbol{R}$ \\
\hline 1 & 1.8542 & 0.0655 & 0.0119 & 0.0664 & 0.03 & 0.19 \\
2 & 1.1016 & 0.0627 & 0.0108 & 0.0634 & 0.03 & 0.18 \\
\hline
\end{tabular}

MgO, Powder, Replicate 2

\begin{tabular}{crrrrrr}
\hline Material & Xbar & $\boldsymbol{s}_{\boldsymbol{x}}$ & $\boldsymbol{s}_{\boldsymbol{r}}$ & $\boldsymbol{s}_{\boldsymbol{R}}$ & $\boldsymbol{r}$ & $\boldsymbol{R}$ \\
\hline 1 & 1.8541 & 0.0663 & 0.0128 & 0.0673 & 0.04 & 0.19 \\
2 & 1.0999 & 0.0625 & 0.0106 & 0.0633 & 0.03 & 0.18 \\
\hline
\end{tabular}

MgO

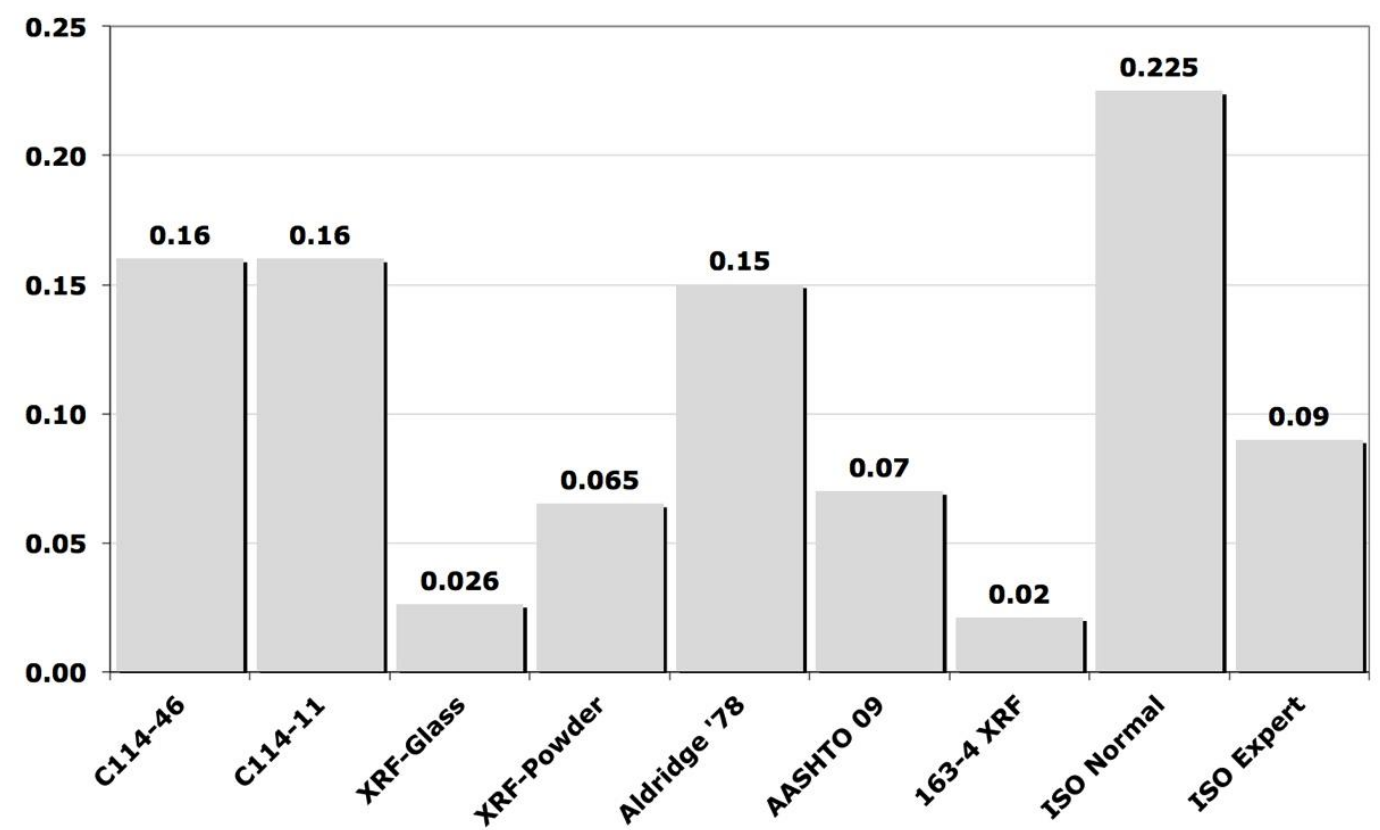

Figure $16 \mathrm{MgO}$ precision statistics by method with bar chart comparing results to current and past ASTM C114 limits and previous studies on chemical analysis precision as 1-sigma, between lab $\left(\mathbf{S}_{\mathbf{R}}\right)$. 
$\mathrm{SO}_{3}$

SO3
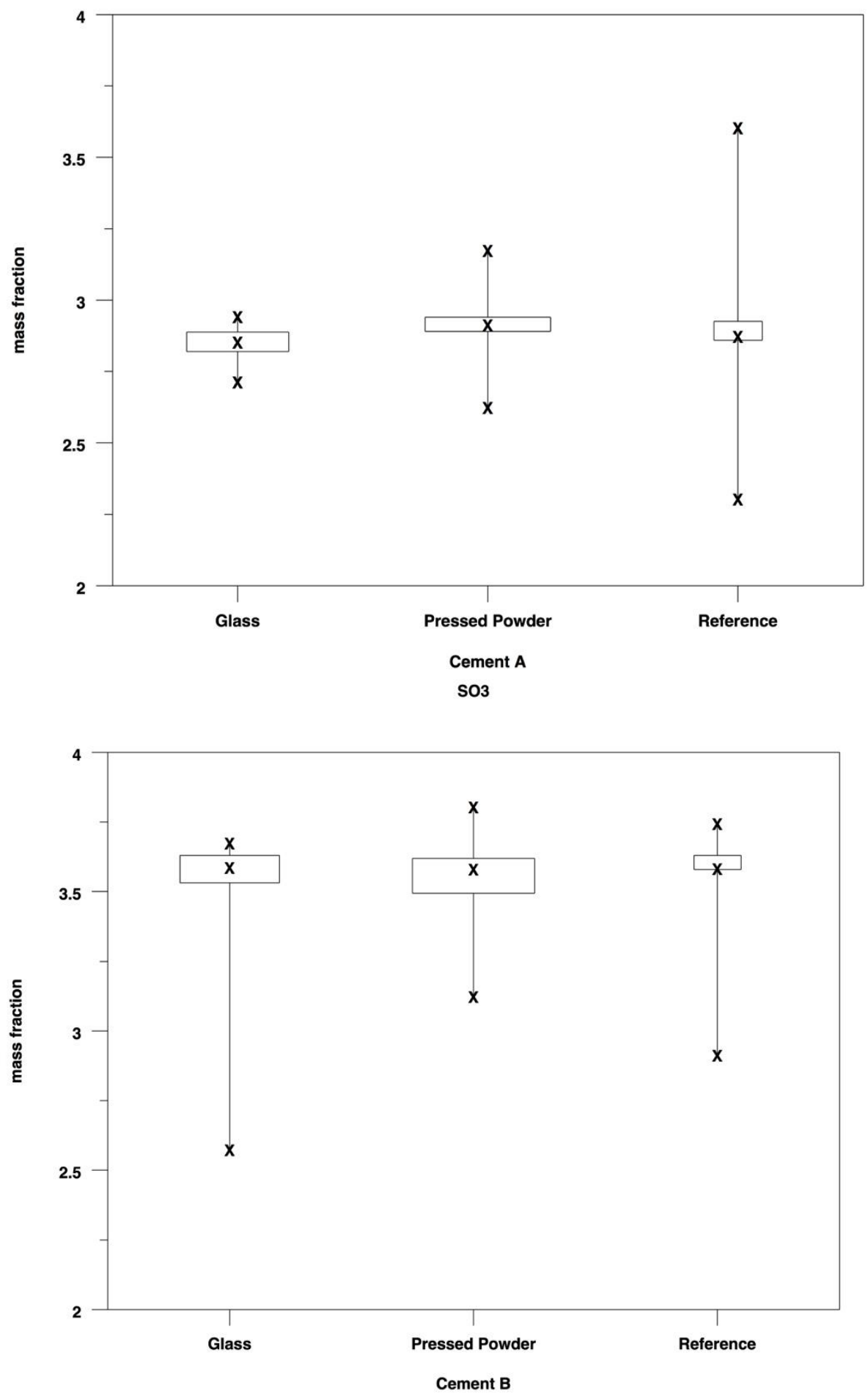

Figure 17 Box plots for $\mathrm{SO}_{3} \mathrm{XRF}$ glass and powder, and reference methods. 
$\mathrm{SO}_{3} \mathrm{XRF}$, Cements A B, Glass, Replicate, Material: 1

\begin{tabular}{crrrrr}
\hline $\begin{array}{c}\text { Laboratory } \\
\text { Number }\end{array}$ & $\begin{array}{r}\text { Cell } \\
\text { Mean }\end{array}$ & $\begin{array}{r}\text { Cell } \\
\text { SD }\end{array}$ & $\boldsymbol{d}$ & $\boldsymbol{h}$ & $\boldsymbol{k}$ \\
\hline 1 & 2.8783 & 0.0223 & 0.0270 & 0.56 & 0.93 \\
2 & 2.8483 & 0.0223 & -0.0030 & -0.06 & 0.93 \\
3 & 2.7650 & 0.0315 & -0.0863 & -1.80 & 1.31 \\
4 & 2.8285 & 0.0244 & -0.0228 & -0.48 & 1.02 \\
5 & 2.8458 & 0.0637 & -0.0055 & -0.11 & 2.66 \\
6 & 2.8382 & 0.0185 & -0.0132 & -0.27 & 0.77 \\
7 & 2.9002 & 0.0141 & 0.0488 & 1.02 & 0.59 \\
8 & 2.8733 & 0.0103 & 0.0220 & 0.46 & 0.43 \\
9 & 2.8038 & 0.0238 & -0.0475 & -0.99 & 0.99 \\
10 & 2.7815 & 0.0191 & -0.0698 & -1.46 & 0.80 \\
11 & 2.9433 & 0.0034 & 0.0920 & 1.92 & 0.14 \\
12 & 2.8883 & 0.0248 & 0.0370 & 0.77 & 1.04 \\
13 & 2.8667 & 0.0121 & 0.0153 & 0.32 & 0.51 \\
14 & 2.9133 & 0.0052 & 0.0620 & 1.29 & 0.22 \\
15 & 2.8150 & 0.0084 & -0.0363 & -0.76 & 0.35 \\
16 & 2.8317 & 0.0098 & -0.0197 & -0.41 & 0.41 \\
\hline
\end{tabular}

Average of cell averages $\quad=\quad 2.85133$

Standard Deviation of cell averages $=0.04798$

Repeatability Standard Deviation $=0.02396$

Reproducibility Standard Deviation $=0.05274$

$\mathrm{h}, \mathrm{k}$ Critical Values $\quad=\quad 2.49000,1.77000$ 
$\mathrm{SO}_{3}$ XRF, Cements A B, Glass, Replicate, Material: 2

\begin{tabular}{crrrrr}
\hline $\begin{array}{c}\text { Laboratory } \\
\text { Number }\end{array}$ & $\begin{array}{r}\text { Cell } \\
\text { Mean }\end{array}$ & $\begin{array}{r}\text { Cell } \\
\text { SD }\end{array}$ & $\boldsymbol{d}$ & $\boldsymbol{h}$ & $\boldsymbol{k}$ \\
\hline 1 & 3.6217 & 0.0133 & 0.0491 & 0.64 & 0.53 \\
2 & 3.5783 & 0.0279 & 0.0057 & 0.07 & 1.11 \\
3 & 3.3883 & 0.0172 & -0.1843 & -2.40 & 0.69 \\
4 & 3.5190 & 0.0125 & -0.0536 & -0.70 & 0.50 \\
5 & 3.5933 & 0.0426 & 0.0207 & 0.27 & 1.70 \\
6 & 3.5367 & 0.0140 & -0.0359 & -0.47 & 0.56 \\
7 & 3.6270 & 0.0406 & 0.0544 & 0.71 & 1.62 \\
8 & 3.6483 & 0.0204 & 0.0757 & 0.99 & 0.81 \\
9 & 3.6090 & 0.0210 & 0.0364 & 0.47 & 0.84 \\
10 & 3.4330 & 0.0477 & -0.1396 & -1.82 & 1.90 \\
11 & 3.6722 & 0.0099 & 0.0996 & 1.30 & 0.40 \\
12 & 3.5700 & 0.0297 & -0.0026 & -0.03 & 1.18 \\
13 & 3.6200 & 0.0190 & 0.0474 & 0.62 & 0.76 \\
14 & 3.6350 & 0.0055 & 0.0624 & 0.81 & 0.22 \\
15 & 3.5283 & 0.0098 & -0.0443 & -0.58 & 0.39 \\
16 & 3.5817 & 0.0194 & 0.0091 & 0.12 & 0.77 \\
\hline
\end{tabular}

Average of cell averages $\quad=\quad 3.57261$

Standard Deviation of cell averages $=0.07685$

Repeatability Standard Deviation $=0.02505$

Reproducibility Standard Deviation $=0.08018$

$\mathrm{h}, \mathrm{k}$ Critical Values $\quad=\quad 2.49000,1.77000$ 
$\mathrm{SO}_{3}$ XRF, Cements A B, Glass, Replicate 2, Material: 1

\begin{tabular}{crrrrr}
\hline $\begin{array}{c}\text { Laboratory } \\
\text { Number }\end{array}$ & $\begin{array}{r}\text { Cell } \\
\text { Mean }\end{array}$ & $\begin{array}{r}\text { Cell } \\
\text { SD }\end{array}$ & $\boldsymbol{d}$ & $\boldsymbol{h}$ & $\boldsymbol{k}$ \\
\hline 1 & 2.8767 & 0.0151 & 0.0251 & 0.52 & 0.66 \\
2 & 2.8500 & 0.0179 & -0.0015 & -0.03 & 0.78 \\
3 & 2.7633 & 0.0403 & -0.0882 & -1.83 & 1.77 \\
4 & 2.8262 & 0.0211 & -0.0254 & -0.53 & 0.93 \\
5 & 2.8500 & 0.0605 & -0.0015 & -0.03 & 2.65 \\
6 & 2.8417 & 0.0238 & -0.0099 & -0.20 & 1.04 \\
7 & 2.9003 & 0.0123 & 0.0488 & 1.01 & 0.54 \\
8 & 2.8783 & 0.0133 & 0.0268 & 0.56 & 0.58 \\
9 & 2.8000 & 0.0116 & -0.0515 & -1.07 & 0.51 \\
10 & 2.7828 & 0.0156 & -0.0687 & -1.43 & 0.69 \\
11 & 2.9417 & 0.0047 & 0.0901 & 1.87 & 0.21 \\
12 & 2.8867 & 0.0151 & 0.0351 & 0.73 & 0.66 \\
13 & 2.8633 & 0.0163 & 0.0118 & 0.25 & 0.72 \\
14 & 2.9150 & 0.0055 & 0.0635 & 1.32 & 0.24 \\
15 & 2.8150 & 0.0105 & -0.0365 & -0.76 & 0.46 \\
16 & 2.8333 & 0.0103 & -0.0182 & -0.38 & 0.45 \\
\hline
\end{tabular}

Average of cell averages $\quad=\quad 2.85152$

Standard Deviation of cell averages $=0.04818$

Repeatability Standard Deviation $=0.02279$

Reproducibility Standard Deviation $=0.05248$

$\mathrm{h}, \mathrm{k}$ Critical Values $\quad=\quad 2.49000,1.77000$ 
$\mathrm{SO}_{3}$ XRF, Cements A B, Glass, Replicate 2, Material: 2

\begin{tabular}{crrrrr}
\hline $\begin{array}{c}\text { Laboratory } \\
\text { Number }\end{array}$ & $\begin{array}{r}\text { Cell } \\
\text { Mean }\end{array}$ & $\begin{array}{r}\text { Cell } \\
\text { SD }\end{array}$ & $\boldsymbol{d}$ & $\boldsymbol{h}$ & $\boldsymbol{k}$ \\
\hline 1 & 3.6333 & 0.0339 & 0.0695 & 0.80 & 0.32 \\
2 & 3.5883 & 0.0299 & 0.0245 & 0.28 & 0.29 \\
3 & 3.3917 & 0.0133 & -0.1722 & -1.97 & 0.13 \\
4 & 3.5223 & 0.0205 & -0.0415 & -0.48 & 0.20 \\
5 & 3.5815 & 0.0448 & 0.0176 & 0.20 & 0.43 \\
6 & 3.5513 & 0.0150 & -0.0125 & -0.14 & 0.14 \\
7 & 3.6283 & 0.0442 & 0.0645 & 0.74 & 0.42 \\
8 & 3.6483 & 0.0075 & 0.0845 & 0.97 & 0.07 \\
9 & 3.6107 & 0.0275 & 0.0468 & 0.54 & 0.26 \\
10 & 3.4338 & 0.0450 & -0.1300 & -1.49 & 0.43 \\
11 & 3.6688 & 0.0101 & 0.1050 & 1.20 & 0.10 \\
12 & 3.4050 & 0.4048 & -0.1589 & -1.82 & 3.88 \\
13 & 3.6183 & 0.0194 & 0.0545 & 0.62 & 0.19 \\
14 & 3.6367 & 0.0082 & 0.0728 & 0.83 & 0.08 \\
15 & 3.5217 & 0.0147 & -0.0422 & -0.48 & 0.14 \\
16 & 3.5817 & 0.0133 & 0.0178 & 0.20 & 0.13 \\
\hline
\end{tabular}

Average of cell averages $\quad=\quad 3.56386$

Standard Deviation of cell averages $=0.08729$

Repeatability Standard Deviation $=0.10441$

Reproducibility Standard Deviation $=0.12924$

$\mathrm{h}, \mathrm{k}$ Critical Values $\quad=\quad 2.49000,1.77000$ 
SO3 XRF, Cements A B, Powder, Replicate 1, Material: 1

\begin{tabular}{crrrrr}
\hline $\begin{array}{c}\text { Laboratory } \\
\text { Number }\end{array}$ & $\begin{array}{r}\text { Cell } \\
\text { Mean }\end{array}$ & $\begin{array}{r}\text { Cell } \\
\text { SD }\end{array}$ & $\boldsymbol{d}$ & $\boldsymbol{h}$ & $\boldsymbol{k}$ \\
\hline 1 & 2.8200 & 0.0179 & -0.0865 & -0.95 & 0.81 \\
2 & 2.7333 & 0.0103 & -0.1731 & -1.90 & 0.47 \\
3 & 2.8917 & 0.0098 & -0.0148 & -0.16 & 0.44 \\
4 & 2.9000 & 0.0155 & -0.0065 & -0.07 & 0.70 \\
5 & 2.9260 & 0.0345 & 0.0195 & 0.21 & 1.56 \\
6 & 2.9267 & 0.0082 & 0.0202 & 0.22 & 0.37 \\
7 & 2.9432 & 0.0473 & 0.0367 & 0.40 & 2.13 \\
8 & 2.9767 & 0.0151 & 0.0702 & 0.77 & 0.68 \\
9 & 2.7558 & 0.0070 & -0.1507 & -1.65 & 0.31 \\
10 & 2.9350 & 0.0187 & 0.0285 & 0.31 & 0.84 \\
11 & 2.9853 & 0.0281 & 0.0789 & 0.86 & 1.26 \\
12 & 2.9065 & 0.0088 & 0.0000 & 0.00 & 0.40 \\
13 & 2.9167 & 0.0242 & 0.0102 & 0.11 & 1.09 \\
14 & 2.9510 & 0.0397 & 0.0445 & 0.49 & 1.79 \\
15 & 2.9083 & 0.0172 & 0.0019 & 0.02 & 0.78 \\
16 & 2.8983 & 0.0117 & -0.0081 & -0.09 & 0.53 \\
17 & 2.9117 & 0.0117 & 0.0052 & 0.06 & 0.53 \\
18 & 2.9149 & 0.0235 & 0.0085 & 0.09 & 1.06 \\
19 & 3.1517 & 0.0232 & 0.2452 & 2.69 & 1.04 \\
20 & 2.9133 & 0.0121 & 0.0069 & 0.08 & 0.55 \\
21 & 2.9259 & 0.0121 & 0.0194 & 0.21 & 0.54 \\
22 & 2.9517 & 0.0183 & 0.0452 & 0.50 & 0.83 \\
23 & 2.9317 & 0.0223 & 0.0252 & 0.28 & 1.00 \\
24 & 2.6800 & 0.0335 & -0.2265 & -2.48 & 1.51 \\
\hline & & & & &
\end{tabular}

Average of cell averages $\quad=\quad 2.90647$

Standard Deviation of cell averages $=0.09120$

Repeatability Standard Deviation $=0.02219$

Reproducibility Standard Deviation $=0.09342$

$\mathrm{h}, \mathrm{k}$ Critical Values $\quad=\quad 2.6000,1.79000$ 
Initial Preparation of Test Result Data for Material: 2

\begin{tabular}{crrrrr}
\hline $\begin{array}{c}\text { Laboratory } \\
\text { Number }\end{array}$ & $\begin{array}{r}\text { Cell } \\
\text { Mean }\end{array}$ & $\begin{array}{r}\text { Cell } \\
\text { SD }\end{array}$ & $\boldsymbol{d}$ & $\boldsymbol{h}$ & $\boldsymbol{k}$ \\
\hline 1 & 3.4583 & 0.0133 & -0.0925 & -0.66 & 0.50 \\
2 & 3.3850 & 0.0084 & -0.1658 & -1.19 & 0.31 \\
3 & 3.5733 & 0.0121 & 0.0225 & 0.16 & 0.45 \\
4 & 3.5833 & 0.0137 & 0.0325 & 0.23 & 0.51 \\
5 & 3.6122 & 0.0229 & 0.0614 & 0.44 & 0.86 \\
6 & 3.5333 & 0.0250 & -0.0175 & -0.13 & 0.94 \\
7 & 3.6287 & 0.0414 & 0.0779 & 0.56 & 1.56 \\
8 & 3.7317 & 0.0264 & 0.1809 & 1.30 & 0.99 \\
9 & 3.3085 & 0.0140 & -0.2423 & -1.74 & 0.53 \\
10 & 3.6217 & 0.0183 & 0.0709 & 0.51 & 0.69 \\
11 & 3.7017 & 0.0380 & 0.1509 & 1.08 & 1.43 \\
12 & 3.6087 & 0.0085 & 0.0579 & 0.42 & 0.32 \\
13 & 3.4567 & 0.0327 & -0.0941 & -0.68 & 1.23 \\
14 & 3.5835 & 0.0240 & 0.0327 & 0.23 & 0.90 \\
15 & 3.6217 & 0.0232 & 0.0709 & 0.51 & 0.87 \\
16 & 3.6167 & 0.0121 & 0.0659 & 0.47 & 0.45 \\
17 & 3.1650 & 0.0259 & -0.3858 & -2.77 & 0.97 \\
18 & 3.6148 & 0.0393 & 0.0640 & 0.46 & 1.48 \\
19 & 3.7883 & 0.0147 & 0.2375 & 1.71 & 0.55 \\
20 & 3.6050 & 0.0295 & 0.0542 & 0.39 & 1.11 \\
21 & 3.5445 & 0.0123 & -0.0063 & -0.05 & 0.46 \\
22 & 3.5450 & 0.0235 & -0.0058 & -0.04 & 0.88 \\
23 & 3.5883 & 0.0382 & 0.0375 & 0.27 & 1.43 \\
24 & 3.3433 & 0.0550 & -0.2075 & -1.49 & 2.07 \\
\hline & & & & &
\end{tabular}

Average of cell averages $\quad=\quad 3.55080$

Standard Deviation of cell averages $=0.13932$

Repeatability Standard Deviation $=0.02662$

Reproducibility Standard Deviation $=0.14142$

$\mathrm{h}, \mathrm{k}$ Critical Values $\quad=\quad 2.6000,1.79000$ 
$\mathrm{SO}_{3}$ XRF, Cements A B, Powder, Replicate 2, Material: 1

\begin{tabular}{crrrrr}
\hline $\begin{array}{c}\text { Laboratory } \\
\text { Number }\end{array}$ & $\begin{array}{r}\text { Cell } \\
\text { Mean }\end{array}$ & $\begin{array}{r}\text { Cell } \\
\text { SD }\end{array}$ & $\boldsymbol{d}$ & $\boldsymbol{h}$ & $\boldsymbol{k}$ \\
\hline 1 & 2.8217 & 0.0194 & -0.0848 & -0.92 & 0.89 \\
2 & 2.7467 & 0.0082 & -0.1598 & -1.74 & 0.38 \\
3 & 2.8983 & 0.0117 & -0.0082 & -0.09 & 0.54 \\
4 & 2.9067 & 0.0151 & 0.0002 & 0.00 & 0.69 \\
5 & 2.9267 & 0.0342 & 0.0202 & 0.22 & 1.57 \\
6 & 2.9267 & 0.0052 & 0.0202 & 0.22 & 0.24 \\
7 & 2.9443 & 0.0487 & 0.0378 & 0.41 & 2.24 \\
8 & 2.9800 & 0.0167 & 0.0735 & 0.80 & 0.77 \\
9 & 2.7500 & 0.0069 & -0.1565 & -1.70 & 0.32 \\
10 & 2.9350 & 0.0105 & 0.0285 & 0.31 & 0.48 \\
11 & 2.9782 & 0.0261 & 0.0717 & 0.78 & 1.20 \\
12 & 2.9080 & 0.0060 & 0.0015 & 0.02 & 0.27 \\
13 & 2.9217 & 0.0223 & 0.0152 & 0.17 & 1.02 \\
14 & 2.9507 & 0.0420 & 0.0442 & 0.48 & 1.93 \\
15 & 2.9067 & 0.0294 & 0.0002 & 0.00 & 1.35 \\
16 & 2.9017 & 0.0075 & -0.0048 & -0.05 & 0.35 \\
17 & 2.9150 & 0.0084 & 0.0085 & 0.09 & 0.38 \\
18 & 2.9170 & 0.0254 & 0.0105 & 0.11 & 1.17 \\
19 & 3.1517 & 0.0223 & 0.2452 & 2.67 & 1.02 \\
20 & 2.9100 & 0.0126 & 0.0035 & 0.04 & 0.58 \\
21 & 2.9260 & 0.0123 & 0.0195 & 0.21 & 0.57 \\
22 & 2.9550 & 0.0187 & 0.0485 & 0.53 & 0.86 \\
23 & 2.9133 & 0.0103 & 0.0068 & 0.07 & 0.47 \\
24 & 2.6650 & 0.0259 & -0.2415 & -2.63 & 1.19 \\
\hline & & & & &
\end{tabular}

Average of cell averages $\quad=\quad 2.90649$

Standard Deviation of cell averages $=0.09196$

Repeatability Standard Deviation $=0.02176$

Reproducibility Standard Deviation $=0.09408$

$\mathrm{h}, \mathrm{k}$ Critical Values $\quad=\quad 2.60000,1.79000$ 
$\mathrm{SO}_{3}$ XRF, Cements A B, Powder, Replicate 2, Material: 2

\begin{tabular}{crrrrr}
\hline $\begin{array}{c}\text { Laboratory } \\
\text { Number }\end{array}$ & $\begin{array}{r}\text { Cell } \\
\text { Mean }\end{array}$ & $\begin{array}{r}\text { Cell } \\
\text { SD }\end{array}$ & $\boldsymbol{d}$ & $\boldsymbol{h}$ & $\boldsymbol{k}$ \\
\hline 1 & 3.4600 & 0.0155 & -0.0921 & -0.67 & 0.64 \\
2 & 3.3983 & 0.0098 & -0.1538 & -1.11 & 0.40 \\
3 & 3.5750 & 0.0176 & 0.0229 & 0.17 & 0.73 \\
4 & 3.5900 & 0.0110 & 0.0379 & 0.27 & 0.45 \\
5 & 3.6128 & 0.0217 & 0.0607 & 0.44 & 0.89 \\
6 & 3.5450 & 0.0308 & -0.0071 & -0.05 & 1.27 \\
7 & 3.6270 & 0.0390 & 0.0749 & 0.54 & 1.60 \\
8 & 3.7317 & 0.0264 & 0.1795 & 1.30 & 1.09 \\
9 & 3.3030 & 0.0164 & -0.2491 & -1.80 & 0.68 \\
10 & 3.6200 & 0.0155 & 0.0679 & 0.49 & 0.64 \\
11 & 3.7005 & 0.0367 & 0.1484 & 1.07 & 1.51 \\
12 & 3.6133 & 0.0061 & 0.0612 & 0.44 & 0.25 \\
13 & 3.4583 & 0.0319 & -0.0938 & -0.68 & 1.31 \\
14 & 3.5858 & 0.0236 & 0.0337 & 0.24 & 0.97 \\
15 & 3.6100 & 0.0141 & 0.0579 & 0.42 & 0.58 \\
16 & 3.6183 & 0.0133 & 0.0662 & 0.48 & 0.55 \\
17 & 3.1617 & 0.0299 & -0.3905 & -2.83 & 1.23 \\
18 & 3.6172 & 0.0382 & 0.0650 & 0.47 & 1.57 \\
19 & 3.7883 & 0.0147 & 0.2362 & 1.71 & 0.61 \\
20 & 3.6050 & 0.0243 & 0.0529 & 0.38 & 1.00 \\
21 & 3.5466 & 0.0137 & -0.0055 & -0.04 & 0.57 \\
22 & 3.5417 & 0.0194 & -0.0105 & -0.08 & 0.80 \\
23 & 3.5800 & 0.0283 & 0.0279 & 0.20 & 1.16 \\
24 & 3.3617 & 0.0371 & -0.1905 & -1.38 & 1.53 \\
\hline & & & & &
\end{tabular}

Average of cell averages $\quad=\quad 3.55214$

Standard Deviation of cell averages $=0.13804$

Repeatability Standard Deviation $=0.02428$

Reproducibility Standard Deviation $=0.13980$

$\mathrm{h}$, k Critical Values $\quad=\quad 2.60000,1.79000$ 
Table 8 Precision Statistics SO3 by XRF glass and powder preparations for replicates 1 and 2.

$\mathrm{SO}_{3}$ Glass, Replicate 1

\begin{tabular}{crrrrrr}
\hline Material & Xbar & $\boldsymbol{s}_{\boldsymbol{x}}$ & $\boldsymbol{s}_{\boldsymbol{r}}$ & $\boldsymbol{s}_{\boldsymbol{R}}$ & $\boldsymbol{r}$ & $\boldsymbol{R}$ \\
\hline 1 & 2.8513 & 0.0480 & 0.0240 & 0.0527 & 0.07 & 0.15 \\
2 & 3.5726 & 0.0769 & 0.0250 & 0.0802 & 0.07 & 0.22 \\
\hline
\end{tabular}

$\mathrm{SO}_{3}$ Glass, Replicate 2

\begin{tabular}{crrrrrr}
\hline Material & Xbar & $\boldsymbol{s}_{\boldsymbol{x}}$ & $\boldsymbol{s}_{\boldsymbol{r}}$ & $\boldsymbol{s}_{\boldsymbol{R}}$ & $\boldsymbol{r}$ & $\boldsymbol{R}$ \\
\hline 1 & 2.8515 & 0.0482 & 0.0228 & 0.0525 & 0.06 & 0.15 \\
2 & 3.5639 & 0.0873 & 0.1044 & 0.1292 & 0.29 & 0.36 \\
\hline
\end{tabular}

$\mathrm{SO}_{3}$ Powder, Replicate 1

\begin{tabular}{crrrrrr}
\hline Material & Xbar & $\boldsymbol{s}_{\boldsymbol{x}}$ & $\boldsymbol{s}_{\boldsymbol{r}}$ & $\boldsymbol{s}_{\boldsymbol{R}}$ & $\boldsymbol{r}$ & $\boldsymbol{R}$ \\
\hline 1 & 2.9065 & 0.0912 & 0.0222 & 0.0934 & 0.06 & 0.26 \\
2 & 3.5508 & 0.1393 & 0.0266 & 0.1414 & 0.07 & 0.40 \\
\hline
\end{tabular}

$\mathrm{SO}_{3}$ Powder, Replicate 2

\begin{tabular}{crrrrrr}
\hline Material & Xbar & $\boldsymbol{s}_{\boldsymbol{x}}$ & $\boldsymbol{s}_{\boldsymbol{r}}$ & $\boldsymbol{s}_{\boldsymbol{R}}$ & $\boldsymbol{r}$ & $\boldsymbol{R}$ \\
\hline 1 & 2.9065 & 0.0920 & 0.0218 & 0.0941 & 0.06 & 0.26 \\
2 & 3.5521 & 0.1380 & 0.0243 & 0.1398 & 0.07 & 0.39 \\
\hline
\end{tabular}

SO3

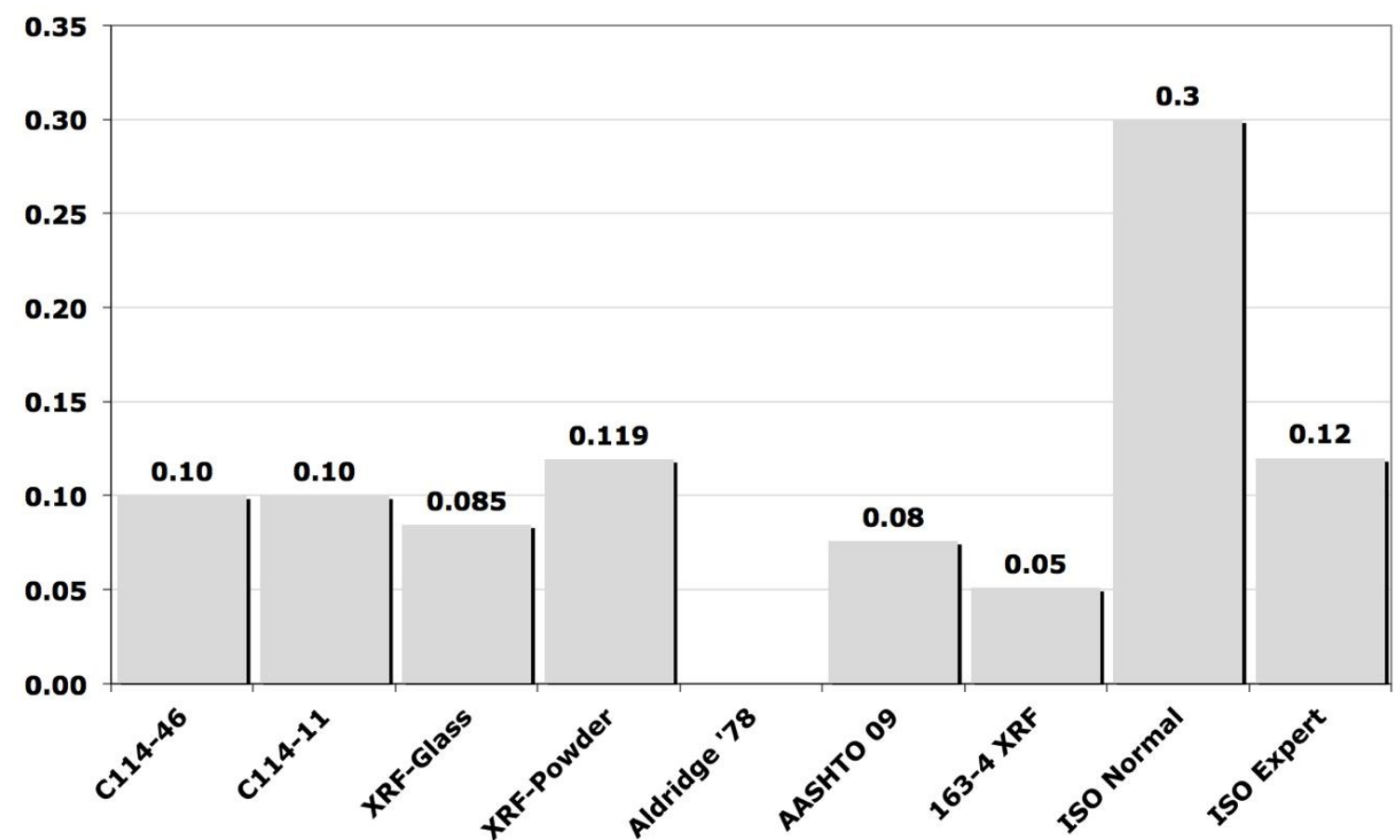

Figure $18 \mathrm{SO}_{3}$ precision statistics by method with bar chart comparing results to current and past ASTM C114 limits and previous studies on chemical analysis precision as 1-sigma, between lab $\left(S_{R}\right)$. 

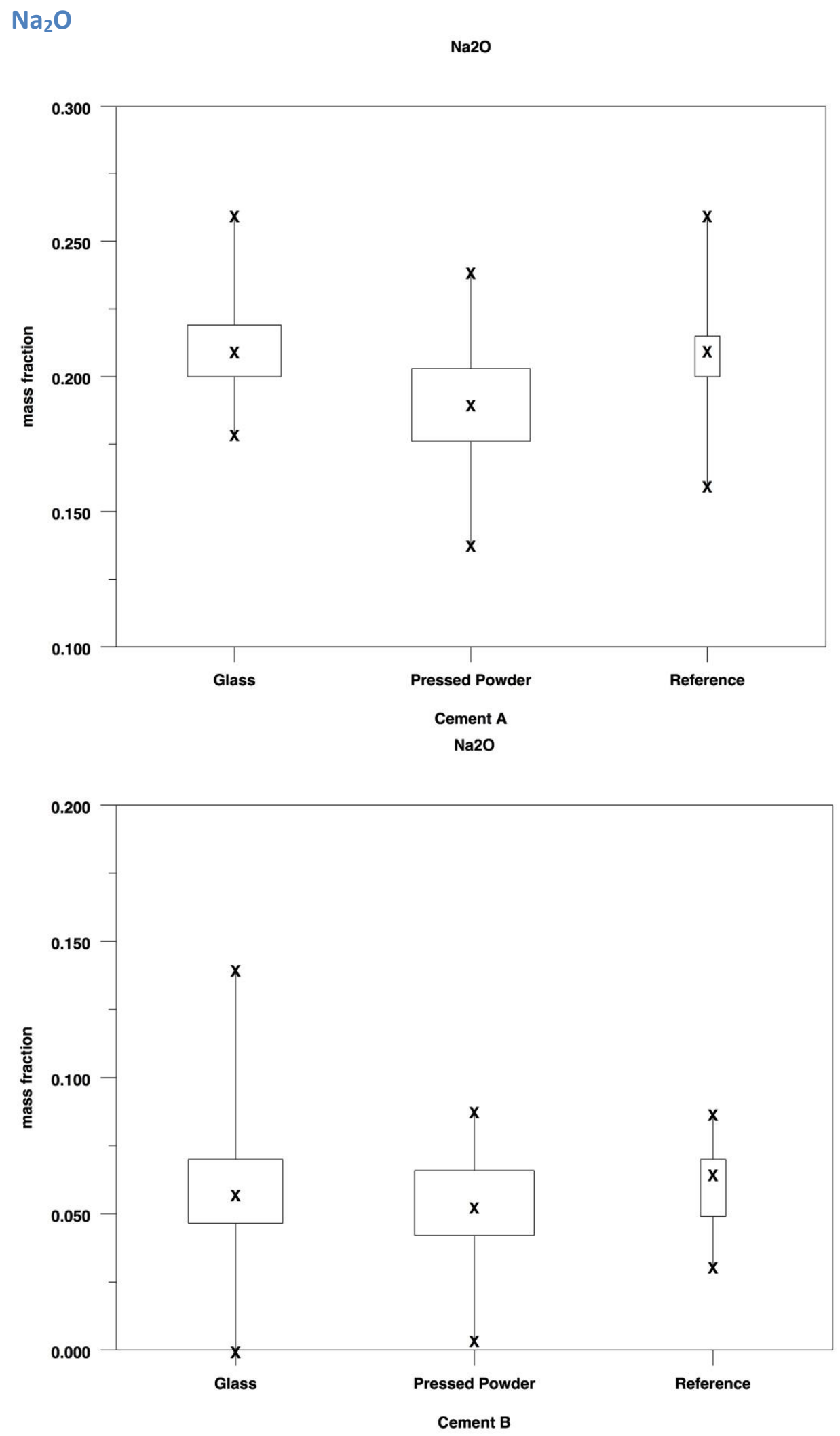

Figure 19 Box plots for $\mathrm{Na}_{2} \mathrm{O}$ XRF glass and powder, and reference methods. 
$\mathrm{Na}_{2} \mathrm{O}$ XRF, Cements A B, Replicate 1, Material: 1

\begin{tabular}{crrrrr}
\hline $\begin{array}{c}\text { Laboratory } \\
\text { Number }\end{array}$ & $\begin{array}{r}\text { Cell } \\
\text { Mean }\end{array}$ & $\begin{array}{r}\text { Cell } \\
\text { SD }\end{array}$ & $\boldsymbol{d}$ & $\boldsymbol{h}$ & $\boldsymbol{k}$ \\
\hline 1 & 0.1950 & 0.0055 & -0.0148 & -1.06 & 0.61 \\
2 & 0.1983 & 0.0041 & -0.0115 & -0.82 & 0.46 \\
3 & 0.2350 & 0.0138 & 0.0252 & 1.80 & 1.55 \\
4 & 0.2083 & 0.0098 & -0.0015 & -0.10 & 1.10 \\
5 & 0.2023 & 0.0057 & -0.0075 & -0.53 & 0.64 \\
6 & 0.2105 & 0.0005 & 0.0007 & 0.05 & 0.05 \\
7 & 0.1867 & 0.0082 & -0.0231 & -1.65 & 0.92 \\
8 & 0.2182 & 0.0065 & 0.0084 & 0.60 & 0.73 \\
9 & 0.2300 & 0.0141 & 0.0202 & 1.44 & 1.59 \\
10 & 0.2056 & 0.0036 & -0.0042 & -0.30 & 0.41 \\
11 & 0.2097 & 0.0051 & -0.0001 & -0.01 & 0.57 \\
12 & 0.2133 & 0.0082 & 0.0035 & 0.25 & 0.92 \\
13 & 0.2293 & 0.0181 & 0.0195 & 1.39 & 2.03 \\
14 & 0.2115 & 0.0052 & 0.0017 & 0.12 & 0.58 \\
15 & 0.1932 & 0.0076 & -0.0166 & -1.19 & 0.85 \\
\hline
\end{tabular}

Average of cell averages $\quad=\quad 0.20979$

Standard Deviation of cell averages $=0.01401$

Repeatability Standard Deviation $=0.00892$

Reproducibility Standard Deviation $=0.01620$

$\mathrm{h}$, k Critical Values $\quad=\quad 2.47000,1.77000$ 
$\mathrm{Na}_{2} \mathrm{O}$ XRF, Cements A B, Replicate 1, Material: 2

\begin{tabular}{crrrrr}
\hline $\begin{array}{c}\text { Laboratory } \\
\text { Number }\end{array}$ & $\begin{array}{r}\text { Cell } \\
\text { Mean }\end{array}$ & $\begin{array}{r}\text { Cell } \\
\text { SD }\end{array}$ & $\boldsymbol{d}$ & $\boldsymbol{h}$ & $\boldsymbol{k}$ \\
\hline 1 & 0.0417 & 0.0117 & -0.0153 & -0.67 & 1.51 \\
2 & 0.0617 & 0.0075 & 0.0047 & 0.21 & 0.97 \\
3 & 0.0967 & 0.0103 & 0.0397 & 1.75 & 1.34 \\
4 & 0.0450 & 0.0055 & -0.0119 & -0.53 & 0.71 \\
5 & 0.0587 & 0.0028 & 0.0017 & 0.08 & 0.36 \\
6 & 0.0556 & 0.0003 & -0.0013 & -0.06 & 0.04 \\
7 & 0.0000 & 0.0000 & -0.0569 & -2.51 & 0.00 \\
8 & 0.0710 & 0.0022 & 0.0141 & 0.62 & 0.28 \\
9 & 0.0900 & 0.0110 & 0.0331 & 1.46 & 1.42 \\
10 & 0.0456 & 0.0041 & -0.0114 & -0.50 & 0.52 \\
11 & 0.0418 & 0.0073 & -0.0151 & -0.66 & 0.94 \\
12 & 0.0633 & 0.0052 & 0.0064 & 0.28 & 0.67 \\
13 & 0.0697 & 0.0156 & 0.0127 & 0.56 & 2.02 \\
14 & 0.0650 & 0.0035 & 0.0081 & 0.36 & 0.45 \\
15 & 0.0482 & 0.0091 & -0.0088 & -0.39 & 1.17 \\
\hline
\end{tabular}

Average of cell averages $\quad=\quad 0.05692$

Standard Deviation of cell averages $=0.02270$

Repeatability Standard Deviation $=0.00773$

Reproducibility Standard Deviation $=0.02378$

$\mathrm{h}, \mathrm{k}$ Critical Values $\quad=\quad 2.47000,1.77000$ 
$\mathrm{Na}_{2} \mathrm{O}$ XRF, Cements A B, Glass, Replicate 2, Material: 1

\begin{tabular}{crrrrr}
\hline $\begin{array}{c}\text { Laboratory } \\
\text { Number }\end{array}$ & $\begin{array}{r}\text { Cell } \\
\text { Mean }\end{array}$ & $\begin{array}{r}\text { Cell } \\
\text { SD }\end{array}$ & $\boldsymbol{d}$ & $\boldsymbol{h}$ & $\boldsymbol{k}$ \\
\hline 1 & 0.1950 & 0.0105 & -0.0148 & -1.18 & 1.26 \\
2 & 0.2000 & 0.0063 & -0.0098 & -0.78 & 0.76 \\
3 & 0.2350 & 0.0138 & 0.0252 & 2.02 & 1.66 \\
4 & 0.2100 & 0.0063 & 0.0002 & 0.02 & 0.76 \\
5 & 0.2023 & 0.0023 & -0.0074 & -0.59 & 0.28 \\
6 & 0.2102 & 0.0009 & 0.0004 & 0.03 & 0.11 \\
7 & 0.1900 & 0.0126 & -0.0198 & -1.58 & 1.52 \\
8 & 0.2190 & 0.0085 & 0.0092 & 0.74 & 1.02 \\
9 & 0.2283 & 0.0117 & 0.0186 & 1.49 & 1.41 \\
10 & 0.2120 & 0.0035 & 0.0022 & 0.18 & 0.43 \\
11 & 0.2057 & 0.0057 & -0.0041 & -0.33 & 0.68 \\
12 & 0.2083 & 0.0075 & -0.0014 & -0.11 & 0.91 \\
13 & 0.2223 & 0.0107 & 0.0126 & 1.01 & 1.29 \\
14 & 0.2115 & 0.0036 & 0.0017 & 0.14 & 0.43 \\
15 & 0.1967 & 0.0073 & -0.0131 & -1.05 & 0.88 \\
\hline
\end{tabular}

Average of cell averages $\quad=\quad 0.20975$

Standard Deviation of cell averages $=0.01249$

Repeatability Standard Deviation $=0.00831$

Reproducibility Standard Deviation $=0.01461$

$\mathrm{h}, \mathrm{k}$ Critical Values $\quad=\quad 2.47000,1.77000$ 
$\mathrm{Na}_{2} \mathrm{O}$ XRF, Cements A B, Glass, Replicate 2, Material: 2

\begin{tabular}{crrrrr}
\hline $\begin{array}{c}\text { Laboratory } \\
\text { Number }\end{array}$ & $\begin{array}{r}\text { Cell } \\
\text { Mean }\end{array}$ & $\begin{array}{r}\text { Cell } \\
\text { SD }\end{array}$ & $\boldsymbol{d}$ & $\boldsymbol{h}$ & $\boldsymbol{k}$ \\
\hline 1 & 0.0450 & 0.0084 & -0.0179 & -0.64 & 0.19 \\
2 & 0.0583 & 0.0075 & -0.0046 & -0.16 & 0.18 \\
3 & 0.1067 & 0.0175 & 0.0437 & 1.56 & 0.41 \\
4 & 0.0467 & 0.0103 & -0.0163 & -0.58 & 0.24 \\
5 & 0.0570 & 0.0025 & -0.0059 & -0.21 & 0.06 \\
6 & 0.0557 & 0.0007 & -0.0072 & -0.26 & 0.02 \\
7 & 0.0000 & 0.0000 & -0.0629 & -2.25 & 0.00 \\
8 & 0.0700 & 0.0027 & 0.0071 & 0.25 & 0.06 \\
9 & 0.0933 & 0.0052 & 0.0304 & 1.09 & 0.12 \\
10 & 0.1141 & 0.1626 & 0.0511 & 1.83 & 3.78 \\
11 & 0.0447 & 0.0080 & -0.0183 & -0.65 & 0.19 \\
12 & 0.0633 & 0.0052 & 0.0004 & 0.01 & 0.12 \\
13 & 0.0777 & 0.0202 & 0.0147 & 0.53 & 0.47 \\
14 & 0.0643 & 0.0028 & 0.0014 & 0.05 & 0.07 \\
15 & 0.0472 & 0.0129 & -0.0158 & -0.56 & 0.30 \\
\hline
\end{tabular}

Average of cell averages $\quad=\quad 0.06293$

Standard Deviation of cell averages $=0.02798$

Repeatability Standard Deviation $=0.04297$

Reproducibility Standard Deviation $=0.04818$

$\mathrm{h}, \mathrm{k}$ Critical Values $\quad=\quad 2.47000,1.77000$ 
$\mathrm{Na}_{2} \mathrm{O}$ XRF, Cements A B, Powder, Replicate 1, Material: 1

\begin{tabular}{crrrrr}
\hline $\begin{array}{c}\text { Laboratory } \\
\text { Number }\end{array}$ & $\begin{array}{r}\text { Cell } \\
\text { Mean }\end{array}$ & $\begin{array}{r}\text { Cell } \\
\text { SD }\end{array}$ & $\boldsymbol{d}$ & $\boldsymbol{h}$ & $\boldsymbol{k}$ \\
\hline 1 & 0.2000 & 0.0000 & 0.0138 & 0.71 & 0.00 \\
2 & 0.1817 & 0.0041 & -0.0045 & -0.23 & 0.82 \\
3 & 0.1773 & 0.0022 & -0.0088 & -0.46 & 0.43 \\
4 & 0.1817 & 0.0117 & -0.0045 & -0.23 & 2.34 \\
5 & 0.2027 & 0.0063 & 0.0165 & 0.85 & 1.26 \\
6 & 0.2000 & 0.0000 & 0.0138 & 0.71 & 0.00 \\
7 & 0.1517 & 0.0041 & -0.0345 & -1.78 & 0.82 \\
8 & 0.2100 & 0.0000 & 0.0238 & 1.23 & 0.00 \\
9 & 0.1883 & 0.0041 & 0.0022 & 0.11 & 0.82 \\
10 & 0.2000 & 0.0063 & 0.0138 & 0.71 & 1.26 \\
11 & 0.2083 & 0.0041 & 0.0222 & 1.14 & 0.82 \\
12 & 0.2055 & 0.0015 & 0.0193 & 1.00 & 0.30 \\
13 & 0.2055 & 0.0024 & 0.0193 & 1.00 & 0.49 \\
14 & 0.1769 & 0.0010 & -0.0093 & -0.48 & 0.20 \\
15 & 0.1475 & 0.0049 & -0.0387 & -2.00 & 0.98 \\
16 & 0.1817 & 0.0041 & -0.0045 & -0.23 & 0.82 \\
17 & 0.1548 & 0.0069 & -0.0313 & -1.62 & 1.37 \\
18 & 0.1758 & 0.0035 & -0.0103 & -0.53 & 0.70 \\
19 & 0.2102 & 0.0071 & 0.0240 & 1.24 & 1.42 \\
20 & 0.1683 & 0.0098 & -0.0178 & -0.92 & 1.96 \\
21 & 0.1877 & 0.0049 & 0.0015 & 0.08 & 0.98 \\
22 & 0.2105 & 0.0019 & 0.0243 & 1.26 & 0.37 \\
23 & 0.1684 & 0.0015 & -0.0177 & -0.91 & 0.29 \\
24 & 0.1733 & 0.0052 & -0.0128 & -0.66 & 1.03 \\
\hline & & & & &
\end{tabular}

Average of cell averages $\quad=\quad 0.18616$

Standard Deviation of cell averages $=0.01936$

Repeatability Standard Deviation $=0.00500$

Reproducibility Standard Deviation $=0.01990$

$\mathrm{h}$, k Critical Values $\quad=\quad 2.60000,1.79000$ 
$\mathrm{Na}_{2} \mathrm{O}$ XRF, Cements A B, Powder, Replicate 1, Material: 2

\begin{tabular}{crrrrr}
\hline $\begin{array}{c}\text { Laboratory } \\
\text { Number }\end{array}$ & $\begin{array}{r}\text { Cell } \\
\text { Mean }\end{array}$ & $\begin{array}{r}\text { Cell } \\
\text { SD }\end{array}$ & $\boldsymbol{d}$ & $\boldsymbol{h}$ & $\boldsymbol{k}$ \\
\hline 1 & 0.0550 & 0.0055 & 0.0024 & 0.13 & 0.92 \\
2 & 0.0517 & 0.0041 & -0.0010 & -0.05 & 0.69 \\
3 & 0.0583 & 0.0052 & 0.0057 & 0.31 & 0.87 \\
4 & 0.0650 & 0.0055 & 0.0124 & 0.68 & 0.92 \\
5 & 0.0687 & 0.0053 & 0.0161 & 0.88 & 0.89 \\
6 & 0.0700 & 0.0000 & 0.0174 & 0.95 & 0.00 \\
7 & 0.0150 & 0.0055 & -0.0376 & -2.07 & 0.92 \\
8 & 0.0600 & 0.0000 & 0.0074 & 0.40 & 0.00 \\
9 & 0.0450 & 0.0055 & -0.0076 & -0.42 & 0.92 \\
10 & 0.0517 & 0.0041 & -0.0010 & -0.05 & 0.69 \\
11 & 0.0750 & 0.0055 & 0.0224 & 1.23 & 0.92 \\
12 & 0.0668 & 0.0015 & 0.0142 & 0.78 & 0.25 \\
13 & 0.0843 & 0.0023 & 0.0317 & 1.74 & 0.39 \\
14 & 0.0457 & 0.0003 & -0.0070 & -0.38 & 0.05 \\
15 & 0.0088 & 0.0038 & -0.0438 & -2.41 & 0.64 \\
16 & 0.0250 & 0.0084 & -0.0276 & -1.52 & 1.41 \\
17 & 0.0482 & 0.0199 & -0.0045 & -0.25 & 3.34 \\
18 & 0.0413 & 0.0023 & -0.0113 & -0.62 & 0.39 \\
19 & 0.0580 & 0.0073 & 0.0054 & 0.29 & 1.23 \\
20 & 0.0467 & 0.0052 & -0.0060 & -0.33 & 0.87 \\
21 & 0.0725 & 0.0047 & 0.0199 & 1.09 & 0.79 \\
22 & 0.0627 & 0.0031 & 0.0100 & 0.55 & 0.52 \\
23 & 0.0479 & 0.0028 & -0.0048 & -0.26 & 0.47 \\
24 & 0.0400 & 0.0000 & -0.0126 & -0.69 & 0.00 \\
\hline & & & & &
\end{tabular}

Average of cell averages $\quad=\quad 0.05264$

Standard Deviation of cell averages $=0.01819$

Repeatability Standard Deviation $=0.00595$

Reproducibility Standard Deviation $=0.01898$

$\mathrm{h}, \mathrm{k}$ Critical Values $\quad=\quad 2.60000,1.79000$ 
$\mathrm{Na}_{2} \mathrm{O}$ XRF, Cements A B, Powder, Replicate 2, Material: 1

\begin{tabular}{crrrrr}
\hline $\begin{array}{c}\text { Laboratory } \\
\text { Number }\end{array}$ & $\begin{array}{r}\text { Cell } \\
\text { Mean }\end{array}$ & $\begin{array}{r}\text { Cell } \\
\text { SD }\end{array}$ & $\boldsymbol{d}$ & $\boldsymbol{h}$ & $\boldsymbol{k}$ \\
\hline 1 & 0.2000 & 0.0000 & 0.0132 & 0.68 & 0.00 \\
2 & 0.1833 & 0.0052 & -0.0035 & -0.18 & 0.98 \\
3 & 0.1783 & 0.0027 & -0.0085 & -0.44 & 0.52 \\
4 & 0.1867 & 0.0052 & -0.0002 & -0.01 & 0.98 \\
5 & 0.2033 & 0.0064 & 0.0165 & 0.85 & 1.21 \\
6 & 0.2000 & 0.0000 & 0.0132 & 0.68 & 0.00 \\
7 & 0.1517 & 0.0041 & -0.0352 & -1.81 & 0.77 \\
8 & 0.2100 & 0.0000 & 0.0232 & 1.19 & 0.00 \\
9 & 0.1900 & 0.0000 & 0.0032 & 0.16 & 0.00 \\
10 & 0.2000 & 0.0063 & 0.0132 & 0.68 & 1.20 \\
11 & 0.2050 & 0.0055 & 0.0182 & 0.94 & 1.04 \\
12 & 0.2058 & 0.0013 & 0.0190 & 0.98 & 0.25 \\
13 & 0.2073 & 0.0023 & 0.0205 & 1.06 & 0.44 \\
14 & 0.1767 & 0.0003 & -0.0102 & -0.52 & 0.05 \\
15 & 0.1490 & 0.0020 & -0.0378 & -1.95 & 0.38 \\
16 & 0.1850 & 0.0055 & -0.0018 & -0.10 & 1.04 \\
17 & 0.1528 & 0.0087 & -0.0340 & -1.75 & 1.64 \\
18 & 0.1757 & 0.0039 & -0.0112 & -0.58 & 0.74 \\
19 & 0.2143 & 0.0132 & 0.0275 & 1.42 & 2.49 \\
20 & 0.1700 & 0.0089 & -0.0168 & -0.87 & 1.69 \\
21 & 0.1870 & 0.0064 & 0.0002 & 0.01 & 1.22 \\
22 & 0.2092 & 0.0031 & 0.0223 & 1.15 & 0.58 \\
23 & 0.1665 & 0.0042 & -0.0203 & -1.05 & 0.80 \\
24 & 0.1767 & 0.0052 & -0.0102 & -0.53 & 0.98 \\
\hline & & & & &
\end{tabular}

Average of cell averages $\quad=\quad 0.18685$

Standard Deviation of cell averages $=0.01938$

Repeatability Standard Deviation $=0.00528$

Reproducibility Standard Deviation $=0.01997$

$\mathrm{h}, \mathrm{k}$ Critical Values $\quad=\quad 2.60000,1.79000$ 
$\mathrm{Na}_{2} \mathrm{O}$ XRF, Cements A B, Powder, Replicate 2, Material: 2

\begin{tabular}{crrrrr}
\hline $\begin{array}{c}\text { Laboratory } \\
\text { Number }\end{array}$ & $\begin{array}{r}\text { Cell } \\
\text { Mean }\end{array}$ & $\begin{array}{r}\text { Cell } \\
\text { SD }\end{array}$ & $\boldsymbol{d}$ & $\boldsymbol{h}$ & $\boldsymbol{k}$ \\
\hline 1 & 0.0550 & 0.0055 & 0.0029 & 0.16 & 1.08 \\
2 & 0.0533 & 0.0052 & 0.0013 & 0.07 & 1.02 \\
3 & 0.0603 & 0.0059 & 0.0083 & 0.46 & 1.16 \\
4 & 0.0633 & 0.0052 & 0.0113 & 0.62 & 1.02 \\
5 & 0.0685 & 0.0066 & 0.0165 & 0.91 & 1.29 \\
6 & 0.0700 & 0.0000 & 0.0179 & 0.99 & 0.00 \\
7 & 0.0150 & 0.0055 & -0.0371 & -2.05 & 1.08 \\
8 & 0.0600 & 0.0000 & 0.0079 & 0.44 & 0.00 \\
9 & 0.0450 & 0.0055 & -0.0071 & -0.39 & 1.08 \\
10 & 0.0517 & 0.0041 & -0.0004 & -0.02 & 0.81 \\
11 & 0.0750 & 0.0055 & 0.0229 & 1.27 & 1.08 \\
12 & 0.0658 & 0.0013 & 0.0138 & 0.76 & 0.26 \\
13 & 0.0835 & 0.0008 & 0.0314 & 1.74 & 0.17 \\
14 & 0.0456 & 0.0002 & -0.0065 & -0.36 & 0.03 \\
15 & 0.0140 & 0.0017 & -0.0381 & -2.11 & 0.33 \\
16 & 0.0250 & 0.0084 & -0.0271 & -1.50 & 1.65 \\
17 & 0.0322 & 0.0137 & -0.0199 & -1.10 & 2.70 \\
18 & 0.0435 & 0.0029 & -0.0086 & -0.47 & 0.58 \\
19 & 0.0590 & 0.0034 & 0.0069 & 0.38 & 0.67 \\
20 & 0.0417 & 0.0041 & -0.0104 & -0.58 & 0.81 \\
21 & 0.0717 & 0.0058 & 0.0196 & 1.08 & 1.15 \\
22 & 0.0632 & 0.0027 & 0.0111 & 0.61 & 0.54 \\
23 & 0.0475 & 0.0026 & -0.0045 & -0.25 & 0.52 \\
24 & 0.0400 & 0.0000 & -0.0121 & -0.67 & 0.00 \\
\hline & & & & &
\end{tabular}

Average of cell averages $\quad=\quad 0.05208$

Standard Deviation of cell averages $=0.01807$

Repeatability Standard Deviation $=0.00506$

Reproducibility Standard Deviation $=0.01865$

$\mathrm{h}, \mathrm{k}$ Critical Values $\quad=\quad 2.60000,1.79000$ 
Table 9 Precision Statistics $\mathrm{Na}_{2} \mathrm{O}$ by XRF glass and powder preparations for replicates 1 and 2.

$\mathrm{Na}_{2} \mathrm{O}$ Glass, Replicate 1

\begin{tabular}{crrrrrr}
\hline Material & Xbar & $\boldsymbol{s}_{\boldsymbol{x}}$ & $\boldsymbol{s}_{\boldsymbol{r}}$ & $\boldsymbol{s}_{\boldsymbol{R}}$ & $\boldsymbol{r}$ & $\boldsymbol{R}$ \\
\hline 1 & 0.2098 & 0.0140 & 0.0089 & 0.0162 & 0.02 & 0.05 \\
2 & 0.0569 & 0.0227 & 0.0077 & 0.0238 & 0.02 & 0.07 \\
\hline
\end{tabular}

$\mathrm{Na}_{2} \mathrm{O}$ Glass, Replicate 2

\begin{tabular}{crrrrrr}
\hline Material & Xbar & $\boldsymbol{s}_{\boldsymbol{x}}$ & $\boldsymbol{s}_{\boldsymbol{r}}$ & $\boldsymbol{s}_{\boldsymbol{R}}$ & $\boldsymbol{r}$ & $\boldsymbol{R}$ \\
\hline 1 & 0.2098 & 0.0125 & 0.0083 & 0.0146 & 0.02 & 0.04 \\
2 & 0.0629 & 0.0280 & 0.0430 & 0.0482 & 0.12 & 0.13 \\
\hline
\end{tabular}

$\mathrm{Na}_{2} \mathrm{O}$ Powder, Replicate 1

\begin{tabular}{crrrrrr}
\hline Material & Xbar & $\boldsymbol{s}_{\boldsymbol{x}}$ & $\boldsymbol{s}_{\boldsymbol{r}}$ & $\boldsymbol{s}_{\boldsymbol{R}}$ & $\boldsymbol{r}$ & $\boldsymbol{R}$ \\
\hline 1 & 0.1862 & 0.0194 & 0.0050 & 0.0199 & 0.01 & 0.06 \\
2 & 0.0526 & 0.0182 & 0.0060 & 0.0190 & 0.02 & 0.05 \\
\hline
\end{tabular}

$\mathrm{Na}_{2} \mathrm{O}$ Powder, Replicate 2

\begin{tabular}{crrrrrr}
\hline Material & Xbar & $\boldsymbol{s}_{\boldsymbol{x}}$ & $\boldsymbol{s}_{\boldsymbol{r}}$ & $\boldsymbol{s}_{\boldsymbol{R}}$ & $\boldsymbol{r}$ & $\boldsymbol{R}$ \\
\hline 1 & 0.1868 & 0.0194 & 0.0053 & 0.0200 & 0.01 & 0.06 \\
2 & 0.0521 & 0.0181 & 0.0051 & 0.0187 & 0.01 & 0.05 \\
\hline
\end{tabular}

\section{Na2O}

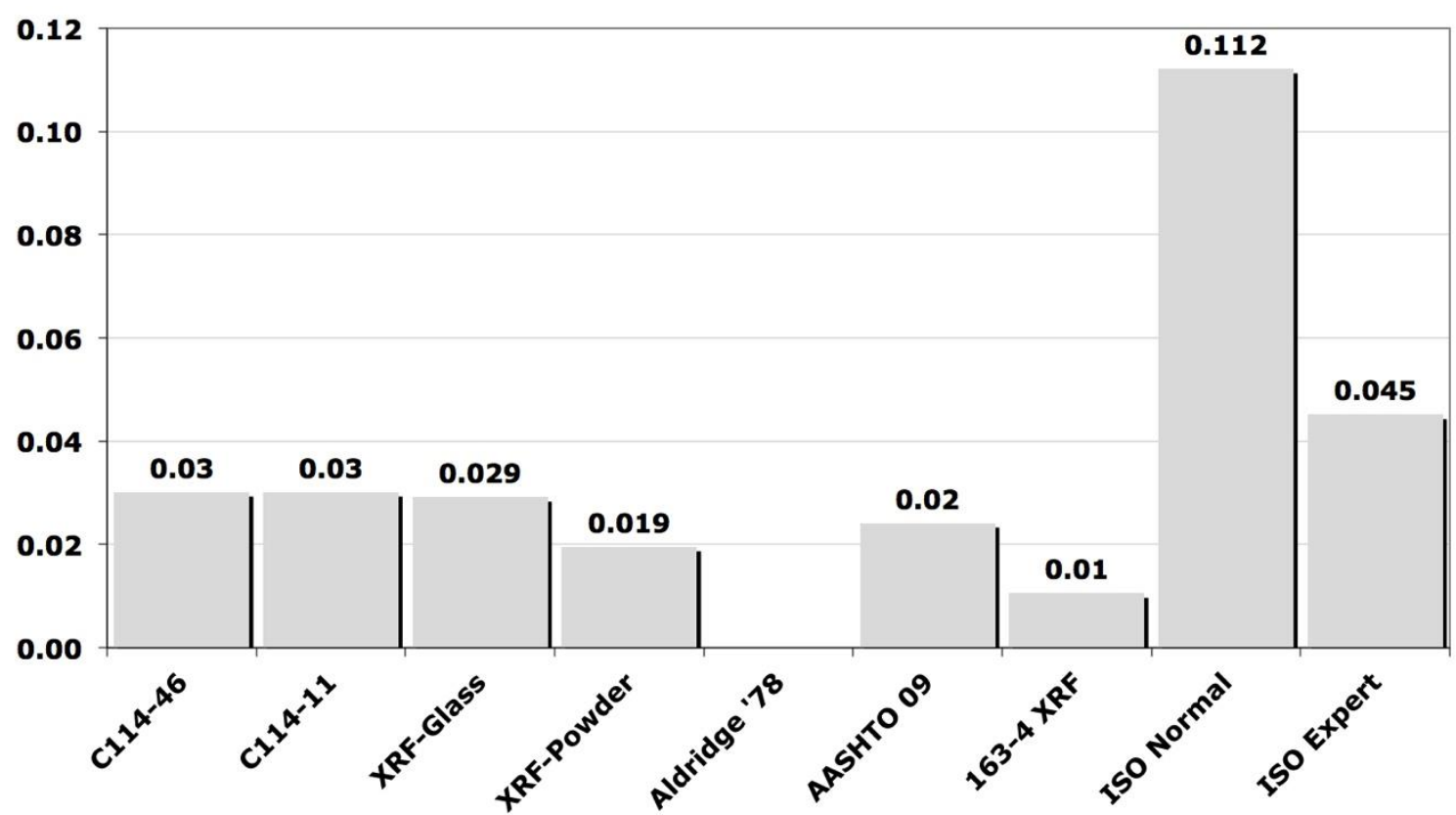

Figure $20 \mathrm{Na}_{2} \mathrm{O}$ precision statistics by method with bar chart comparing results to current and past ASTM C114 limits and previous studies on chemical analysis precision as 1-sigma, between lab $\left(S_{R}\right)$. 
$\mathrm{K}_{2} \mathrm{O}$

K2O
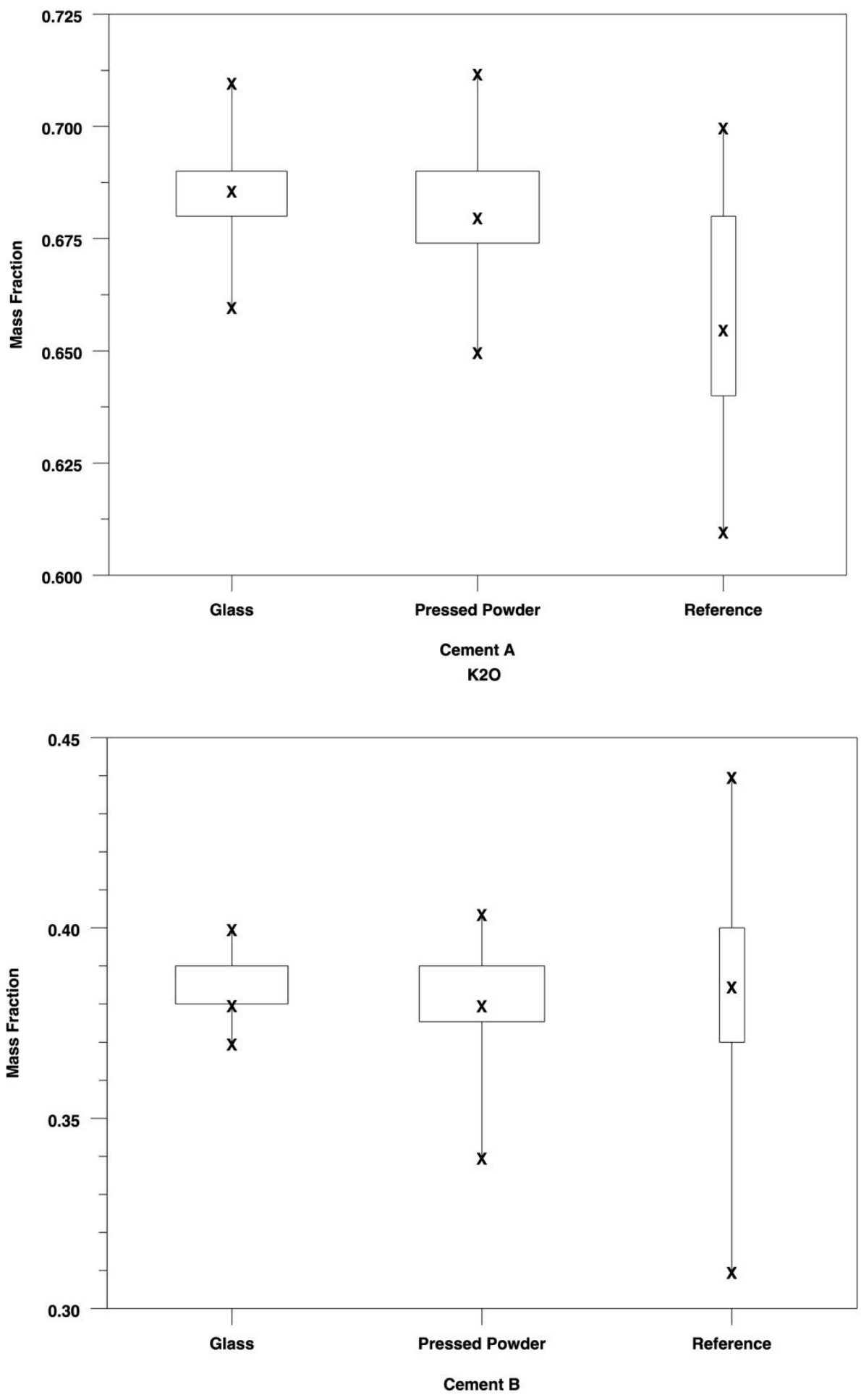

Figure 21 Box plots for $\mathrm{K}_{2} \mathrm{O}$ XRF glass and powder, and reference methods. 
$\mathrm{K}_{2} \mathrm{O}$ XRF, Cements A B, Glass, Replicate 1, Material 1

\begin{tabular}{crrrrr}
\hline $\begin{array}{c}\text { Laboratory } \\
\text { Number }\end{array}$ & $\begin{array}{r}\text { Cell } \\
\text { Mean }\end{array}$ & $\begin{array}{r}\text { Cell } \\
\text { SD }\end{array}$ & $\boldsymbol{d}$ & $\boldsymbol{h}$ & $\boldsymbol{k}$ \\
\hline 1 & 0.6800 & 0.0000 & -0.0064 & -0.67 & 0.00 \\
2 & 0.6800 & 0.0000 & -0.0064 & -0.67 & 0.00 \\
3 & 0.6878 & 0.0042 & 0.0014 & 0.14 & 0.97 \\
4 & 0.7067 & 0.0052 & 0.0202 & 2.09 & 1.21 \\
5 & 0.6917 & 0.0041 & 0.0052 & 0.54 & 0.95 \\
6 & 0.6817 & 0.0075 & -0.0048 & -0.49 & 1.76 \\
7 & 0.6985 & 0.0020 & 0.0121 & 1.25 & 0.47 \\
8 & 0.6767 & 0.0028 & -0.0098 & -1.01 & 0.65 \\
9 & 0.7050 & 0.0055 & 0.0186 & 1.92 & 1.28 \\
10 & 0.6742 & 0.0060 & -0.0123 & -1.27 & 1.40 \\
11 & 0.6850 & 0.0055 & -0.0014 & -0.15 & 1.28 \\
12 & 0.6834 & 0.0019 & -0.0031 & -0.32 & 0.44 \\
13 & 0.6897 & 0.0015 & 0.0032 & 0.33 & 0.35 \\
14 & 0.6883 & 0.0041 & 0.0019 & 0.20 & 0.95 \\
15 & 0.6843 & 0.0033 & -0.0021 & -0.22 & 0.76 \\
16 & 0.6733 & 0.0052 & -0.0131 & -1.36 & 1.21 \\
17 & 0.6833 & 0.0052 & -0.0031 & -0.32 & 1.21 \\
\hline
\end{tabular}

Average of cell averages $\quad=\quad 0.68645$

Standard Deviation of cell averages $=0.00966$

Repeatability Standard Deviation $=0.00428$

Reproducibility Standard Deviation $=0.01042$

$\mathrm{h}, \mathrm{k}$ Critical Values $\quad=\quad 2.51000,1.78000$ 
$\mathrm{K}_{2} \mathrm{O}$ XRF, Cements A B, Glass, Replicate 1, Material: 2

\begin{tabular}{crrrrr}
\hline $\begin{array}{c}\text { Laboratory } \\
\text { Number }\end{array}$ & $\begin{array}{r}\text { Cell } \\
\text { Mean }\end{array}$ & $\begin{array}{r}\text { Cell } \\
\text { SD }\end{array}$ & $\boldsymbol{d}$ & $\boldsymbol{h}$ & $\boldsymbol{k}$ \\
\hline 1 & 0.3817 & 0.0041 & -0.0017 & -0.33 & 1.16 \\
2 & 0.3800 & 0.0000 & -0.0034 & -0.66 & 0.00 \\
3 & 0.3890 & 0.0022 & 0.0056 & 1.10 & 0.62 \\
4 & 0.3850 & 0.0055 & 0.0016 & 0.32 & 1.56 \\
5 & 0.3917 & 0.0041 & 0.0083 & 1.62 & 1.16 \\
6 & 0.3800 & 0.0000 & -0.0034 & -0.66 & 0.00 \\
7 & 0.3932 & 0.0013 & 0.0098 & 1.92 & 0.36 \\
8 & 0.3808 & 0.0017 & -0.0025 & -0.49 & 0.49 \\
9 & 0.3850 & 0.0055 & 0.0016 & 0.32 & 1.56 \\
10 & 0.3828 & 0.0044 & -0.0005 & -0.10 & 1.24 \\
11 & 0.3817 & 0.0041 & -0.0017 & -0.33 & 1.16 \\
12 & 0.3782 & 0.0014 & -0.0052 & -1.02 & 0.41 \\
13 & 0.3862 & 0.0017 & 0.0028 & 0.55 & 0.49 \\
14 & 0.3883 & 0.0041 & 0.0050 & 0.97 & 1.16 \\
15 & 0.3803 & 0.0048 & -0.0030 & -0.59 & 1.37 \\
16 & 0.3733 & 0.0052 & -0.0100 & -1.96 & 1.47 \\
17 & 0.3800 & 0.0000 & -0.0034 & -0.66 & 0.00 \\
\hline
\end{tabular}

Average of cell averages $\quad=0.38336$

Standard Deviation of cell averages $=0.00511$

Repeatability Standard Deviation $=0.00351$

Reproducibility Standard Deviation $=0.00604$

$\mathrm{h}$, k Critical Values $\quad=\quad 2.51000,1.78000$ 
$\mathrm{K}_{2} \mathrm{O}$ XRF, Cements A B, Glass, Replicate 2, Material: 1

\begin{tabular}{crrrrr}
\hline $\begin{array}{c}\text { Laboratory } \\
\text { Number }\end{array}$ & $\begin{array}{r}\text { Cell } \\
\text { Mean }\end{array}$ & $\begin{array}{r}\text { Cell } \\
\text { SD }\end{array}$ & $\boldsymbol{d}$ & $\boldsymbol{h}$ & $\boldsymbol{k}$ \\
\hline 1 & 0.6733 & 0.0082 & -0.0130 & -1.29 & 1.70 \\
2 & 0.6800 & 0.0000 & -0.0064 & -0.63 & 0.00 \\
3 & 0.6900 & 0.0037 & 0.0036 & 0.36 & 0.78 \\
4 & 0.7067 & 0.0052 & 0.0203 & 2.00 & 1.07 \\
5 & 0.6933 & 0.0052 & 0.0070 & 0.69 & 1.07 \\
6 & 0.6833 & 0.0052 & -0.0030 & -0.30 & 1.07 \\
7 & 0.6976 & 0.0023 & 0.0112 & 1.10 & 0.49 \\
8 & 0.6755 & 0.0052 & -0.0109 & -1.07 & 1.08 \\
9 & 0.7050 & 0.0055 & 0.0186 & 1.84 & 1.14 \\
10 & 0.6757 & 0.0067 & -0.0107 & -1.06 & 1.39 \\
11 & 0.6850 & 0.0055 & -0.0014 & -0.14 & 1.14 \\
12 & 0.6835 & 0.0016 & -0.0029 & -0.28 & 0.34 \\
13 & 0.6900 & 0.0021 & 0.0036 & 0.36 & 0.44 \\
14 & 0.6867 & 0.0052 & 0.0003 & 0.03 & 1.07 \\
15 & 0.6845 & 0.0042 & -0.0019 & -0.19 & 0.87 \\
16 & 0.6717 & 0.0041 & -0.0147 & -1.45 & 0.85 \\
17 & 0.6867 & 0.0052 & 0.0003 & 0.03 & 1.07 \\
\hline
\end{tabular}

Average of cell averages $\quad=\quad 0.68638$

Standard Deviation of cell averages $=0.01013$

Repeatability Standard Deviation $=0.00480$

Reproducibility Standard Deviation $=0.01104$

$\mathrm{h}, \mathrm{k}$ Critical Values $\quad=\quad 2.51000,1.78000$ 
$\mathrm{K}_{2} \mathrm{O}$ XRF, Cements A B, Glass, Replicate 2, Material: 2

\begin{tabular}{crrrrr}
\hline $\begin{array}{c}\text { Laboratory } \\
\text { Number }\end{array}$ & $\begin{array}{r}\text { Cell } \\
\text { Mean }\end{array}$ & $\begin{array}{r}\text { Cell } \\
\text { SD }\end{array}$ & $\boldsymbol{d}$ & $\boldsymbol{h}$ & $\boldsymbol{k}$ \\
\hline 1 & 0.3800 & 0.0000 & -0.0030 & -0.58 & 0.00 \\
2 & 0.3800 & 0.0000 & -0.0030 & -0.58 & 0.00 \\
3 & 0.3882 & 0.0022 & 0.0052 & 1.00 & 0.65 \\
4 & 0.3883 & 0.0041 & 0.0053 & 1.03 & 1.18 \\
5 & 0.3900 & 0.0000 & 0.0070 & 1.35 & 0.00 \\
6 & 0.3800 & 0.0000 & -0.0030 & -0.58 & 0.00 \\
7 & 0.3930 & 0.0010 & 0.0100 & 1.94 & 0.28 \\
8 & 0.3800 & 0.0017 & -0.0030 & -0.58 & 0.49 \\
9 & 0.3850 & 0.0055 & 0.0020 & 0.38 & 1.59 \\
10 & 0.3812 & 0.0056 & -0.0018 & -0.36 & 1.63 \\
11 & 0.3800 & 0.0063 & -0.0030 & -0.58 & 1.83 \\
12 & 0.3785 & 0.0016 & -0.0045 & -0.86 & 0.46 \\
13 & 0.3858 & 0.0017 & 0.0028 & 0.54 & 0.50 \\
14 & 0.3883 & 0.0041 & 0.0053 & 1.03 & 1.18 \\
15 & 0.3795 & 0.0051 & -0.0035 & -0.68 & 1.49 \\
16 & 0.3733 & 0.0052 & -0.0097 & -1.87 & 1.50 \\
17 & 0.3800 & 0.0000 & -0.0030 & -0.58 & 0.00 \\
\hline
\end{tabular}

Average of cell averages $=0.38301$

Standard Deviation of cell averages $=0.00517$

Repeatability Standard Deviation $=0.00345$

Reproducibility Standard Deviation $=0.00606$

$\mathrm{h}, \mathrm{k}$ Critical Values $\quad=\quad 2.51000,1.78000$ 
$\mathrm{K}_{2} \mathrm{O}$ XRF, Cements A B, Powder, Replicate 1, Material: 1

\begin{tabular}{crrrrr}
\hline $\begin{array}{c}\text { Laboratory } \\
\text { Number }\end{array}$ & $\begin{array}{r}\text { Cell } \\
\text { Mean }\end{array}$ & $\begin{array}{r}\text { Cell } \\
\text { SD }\end{array}$ & $\boldsymbol{d}$ & $\boldsymbol{h}$ & $\boldsymbol{k}$ \\
\hline 1 & 0.6732 & 0.0008 & -0.0092 & -0.75 & 0.16 \\
2 & 0.6617 & 0.0041 & -0.0207 & -1.69 & 0.86 \\
3 & 0.7022 & 0.0033 & 0.0198 & 1.61 & 0.69 \\
4 & 0.6800 & 0.0000 & -0.0024 & -0.19 & 0.00 \\
5 & 0.6767 & 0.0052 & -0.0057 & -0.47 & 1.09 \\
6 & 0.6767 & 0.0052 & -0.0057 & -0.47 & 1.09 \\
7 & 0.6900 & 0.0063 & 0.0076 & 0.62 & 1.34 \\
8 & 0.6900 & 0.0063 & 0.0076 & 0.62 & 1.34 \\
9 & 0.6833 & 0.0052 & 0.0009 & 0.08 & 1.09 \\
10 & 0.6833 & 0.0052 & 0.0009 & 0.08 & 1.09 \\
11 & 0.6800 & 0.0000 & -0.0024 & -0.19 & 0.00 \\
12 & 0.6733 & 0.0052 & -0.0091 & -0.74 & 1.09 \\
13 & 0.6824 & 0.0059 & 0.0000 & 0.00 & 1.26 \\
14 & 0.7083 & 0.0041 & 0.0259 & 2.11 & 0.86 \\
15 & 0.6562 & 0.0010 & -0.0262 & -2.13 & 0.21 \\
16 & 0.6972 & 0.0078 & 0.0148 & 1.20 & 1.65 \\
17 & 0.6883 & 0.0075 & 0.0059 & 0.48 & 1.59 \\
18 & 0.6738 & 0.0021 & -0.0086 & -0.70 & 0.45 \\
19 & 0.6750 & 0.0055 & -0.0074 & -0.60 & 1.16 \\
20 & 0.6901 & 0.0024 & 0.0077 & 0.63 & 0.51 \\
21 & 0.6883 & 0.0041 & 0.0059 & 0.48 & 0.86 \\
\hline & & & & &
\end{tabular}

Average of cell averages $\quad=\quad 0.68238$

Standard Deviation of cell averages $=0.01228$

Repeatability Standard Deviation $=0.00473$

Reproducibility Standard Deviation $=0.01302$

$\mathrm{h}, \mathrm{k}$ Critical Values $\quad=\quad 2.57000,1.79000$ 
$\mathrm{K}_{2} \mathrm{O}$ XRF, Cements A B, Powder, Replicate 1, Material: 2

\begin{tabular}{crrrrr}
\hline $\begin{array}{c}\text { Laboratory } \\
\text { Number }\end{array}$ & $\begin{array}{r}\text { Cell } \\
\text { Mean }\end{array}$ & $\begin{array}{r}\text { Cell } \\
\text { SD }\end{array}$ & $\boldsymbol{d}$ & $\boldsymbol{h}$ & $\boldsymbol{k}$ \\
\hline 1 & 0.3782 & 0.0030 & -0.0019 & -0.18 & 0.78 \\
2 & 0.3800 & 0.0000 & -0.0001 & -0.01 & 0.00 \\
3 & 0.3935 & 0.0012 & 0.0134 & 1.28 & 0.32 \\
4 & 0.3800 & 0.0000 & -0.0001 & -0.01 & 0.00 \\
5 & 0.3483 & 0.0041 & -0.0317 & -3.03 & 1.06 \\
6 & 0.3700 & 0.0000 & -0.0101 & -0.96 & 0.00 \\
7 & 0.3717 & 0.0041 & -0.0084 & -0.80 & 1.06 \\
8 & 0.3833 & 0.0052 & 0.0033 & 0.31 & 1.35 \\
9 & 0.3817 & 0.0075 & 0.0016 & 0.15 & 1.96 \\
10 & 0.3833 & 0.0052 & 0.0033 & 0.31 & 1.35 \\
11 & 0.3700 & 0.0000 & -0.0101 & -0.96 & 0.00 \\
12 & 0.3883 & 0.0041 & 0.0083 & 0.79 & 1.06 \\
13 & 0.3737 & 0.0015 & -0.0064 & -0.61 & 0.39 \\
14 & 0.3933 & 0.0052 & 0.0133 & 1.26 & 1.35 \\
15 & 0.3761 & 0.0008 & -0.0040 & -0.38 & 0.21 \\
16 & 0.3955 & 0.0057 & 0.0154 & 1.47 & 1.48 \\
17 & 0.3883 & 0.0041 & 0.0083 & 0.79 & 1.06 \\
18 & 0.3825 & 0.0032 & 0.0024 & 0.23 & 0.84 \\
19 & 0.3883 & 0.0041 & 0.0083 & 0.79 & 1.06 \\
20 & 0.3805 & 0.0018 & 0.0005 & 0.04 & 0.48 \\
21 & 0.3750 & 0.0055 & -0.0051 & -0.48 & 1.43 \\
\hline
\end{tabular}

Average of cell averages $\quad=0.38008$

Standard Deviation of cell averages $=0.01048$

Repeatability Standard Deviation $=0.00384$

Reproducibility Standard Deviation $=0.01105$

$\mathrm{h}, \mathrm{k}$ Critical Values $\quad=\quad 2.57000,1.79000$ 
$\mathrm{K}_{2} \mathrm{O}$ XRF, Cements A B, Powder, Replicate 2, Material: 1

\begin{tabular}{crrrrr}
\hline $\begin{array}{c}\text { Laboratory } \\
\text { Number }\end{array}$ & $\begin{array}{r}\text { Cell } \\
\text { Mean }\end{array}$ & $\begin{array}{r}\text { Cell } \\
\text { SD }\end{array}$ & $\boldsymbol{d}$ & $\boldsymbol{h}$ & $\boldsymbol{k}$ \\
\hline 1 & 0.6728 & 0.0010 & -0.0095 & -0.76 & 0.21 \\
2 & 0.6583 & 0.0041 & -0.0240 & -1.92 & 0.87 \\
3 & 0.7022 & 0.0029 & 0.0198 & 1.58 & 0.62 \\
4 & 0.6800 & 0.0000 & -0.0023 & -0.19 & 0.00 \\
5 & 0.6767 & 0.0052 & -0.0057 & -0.45 & 1.10 \\
6 & 0.6783 & 0.0041 & -0.0040 & -0.32 & 0.87 \\
7 & 0.6900 & 0.0063 & 0.0077 & 0.61 & 1.34 \\
8 & 0.6883 & 0.0041 & 0.0060 & 0.48 & 0.87 \\
9 & 0.6833 & 0.0082 & 0.0010 & 0.08 & 1.73 \\
10 & 0.6867 & 0.0052 & 0.0043 & 0.35 & 1.10 \\
11 & 0.6800 & 0.0000 & -0.0023 & -0.19 & 0.00 \\
12 & 0.6750 & 0.0055 & -0.0073 & -0.59 & 1.16 \\
13 & 0.6823 & 0.0064 & 0.0000 & 0.00 & 1.36 \\
14 & 0.7083 & 0.0041 & 0.0260 & 2.08 & 0.87 \\
15 & 0.6552 & 0.0006 & -0.0271 & -2.17 & 0.13 \\
16 & 0.6975 & 0.0072 & 0.0152 & 1.21 & 1.54 \\
17 & 0.6900 & 0.0063 & 0.0077 & 0.61 & 1.34 \\
18 & 0.6743 & 0.0026 & -0.0080 & -0.64 & 0.55 \\
19 & 0.6767 & 0.0052 & -0.0057 & -0.45 & 1.10 \\
20 & 0.6896 & 0.0023 & 0.0073 & 0.58 & 0.49 \\
21 & 0.6833 & 0.0052 & 0.0010 & 0.08 & 1.10 \\
\hline & & & & &
\end{tabular}

Average of cell averages $\quad=\quad 0.68233$

Standard Deviation of cell averages $=0.01252$

Repeatability Standard Deviation $=0.00471$

Reproducibility Standard Deviation $=0.01324$

$\mathrm{h}, \mathrm{k}$ Critical Values $\quad=\quad 2.57000,1.79000$ 
$\mathrm{K}_{2} \mathrm{O}$ XRF, Cements A B, Powder, Replicate 2, Material: 2

\begin{tabular}{crrrrr}
\hline $\begin{array}{c}\text { Laboratory } \\
\text { Number }\end{array}$ & $\begin{array}{r}\text { Cell } \\
\text { Mean }\end{array}$ & $\begin{array}{r}\text { Cell } \\
\text { SD }\end{array}$ & $\boldsymbol{d}$ & $\boldsymbol{h}$ & $\boldsymbol{k}$ \\
\hline 1 & 0.3782 & 0.0026 & -0.0019 & -0.19 & 0.74 \\
2 & 0.3783 & 0.0041 & -0.0017 & -0.17 & 1.14 \\
3 & 0.3937 & 0.0012 & 0.0136 & 1.32 & 0.34 \\
4 & 0.3800 & 0.0000 & -0.0001 & -0.01 & 0.00 \\
5 & 0.3483 & 0.0041 & -0.0317 & -3.08 & 1.14 \\
6 & 0.3700 & 0.0000 & -0.0101 & -0.98 & 0.00 \\
7 & 0.3717 & 0.0041 & -0.0084 & -0.82 & 1.14 \\
8 & 0.3833 & 0.0052 & 0.0033 & 0.32 & 1.45 \\
9 & 0.3817 & 0.0041 & 0.0016 & 0.15 & 1.14 \\
10 & 0.3833 & 0.0052 & 0.0033 & 0.32 & 1.45 \\
11 & 0.3700 & 0.0000 & -0.0101 & -0.98 & 0.00 \\
12 & 0.3867 & 0.0052 & 0.0066 & 0.64 & 1.45 \\
13 & 0.3735 & 0.0020 & -0.0066 & -0.64 & 0.56 \\
14 & 0.3933 & 0.0052 & 0.0133 & 1.29 & 1.45 \\
15 & 0.3760 & 0.0007 & -0.0041 & -0.40 & 0.20 \\
16 & 0.3952 & 0.0056 & 0.0151 & 1.46 & 1.56 \\
17 & 0.3867 & 0.0052 & 0.0066 & 0.64 & 1.45 \\
18 & 0.3832 & 0.0022 & 0.0031 & 0.30 & 0.62 \\
19 & 0.3883 & 0.0041 & 0.0083 & 0.80 & 1.14 \\
20 & 0.3803 & 0.0016 & 0.0002 & 0.02 & 0.45 \\
21 & 0.3800 & 0.0000 & -0.0001 & -0.01 & 0.00 \\
\hline & & & & &
\end{tabular}

Average of cell averages $\quad=0.38008$

Standard Deviation of cell averages $=0.01031$

Repeatability Standard Deviation $=0.00357$

Reproducibility Standard Deviation $=0.01081$

$\mathrm{h}, \mathrm{k}$ Critical Values $\quad=\quad 2.57000,1.79000$ 
Table 10 Precision Statistics $\mathrm{K}_{2} \mathrm{O}$ by XRF glass and powder preparations for replicates 1 and 2.

$\mathrm{K}_{2} \mathrm{O}$ Glass, Replicate 1

\begin{tabular}{crrrrrr}
\hline Material & Xbar & $\boldsymbol{s}_{\boldsymbol{x}}$ & $\boldsymbol{s}_{\boldsymbol{r}}$ & $\boldsymbol{s}_{\boldsymbol{R}}$ & $\boldsymbol{r}$ & $\boldsymbol{R}$ \\
\hline 1 & 0.6864 & 0.0097 & 0.0043 & 0.0104 & 0.01 & 0.03 \\
2 & 0.3834 & 0.0051 & 0.0035 & 0.0060 & 0.01 & 0.02 \\
\hline
\end{tabular}

$\mathrm{K}_{2} \mathrm{O}$ Glass, Replicate 2

\begin{tabular}{crrrrrr}
\hline Material & Xbar & $\boldsymbol{s}_{\boldsymbol{x}}$ & $\boldsymbol{s}_{\boldsymbol{r}}$ & $\boldsymbol{s}_{\boldsymbol{R}}$ & $\boldsymbol{r}$ & $\boldsymbol{R}$ \\
\hline 1 & 0.6864 & 0.0101 & 0.0048 & 0.0110 & 0.01 & 0.03 \\
2 & 0.3830 & 0.0052 & 0.0034 & 0.0061 & 0.01 & 0.02 \\
\hline
\end{tabular}

$\mathrm{K}_{2} \mathrm{O}$ Powder, Replicate 1

\begin{tabular}{crrrrrr}
\hline Material & Xbar & $\boldsymbol{s}_{\boldsymbol{x}}$ & $\boldsymbol{s}_{\boldsymbol{r}}$ & $\boldsymbol{s}_{\boldsymbol{R}}$ & $\boldsymbol{r}$ & $\boldsymbol{R}$ \\
\hline 1 & 0.6824 & 0.0123 & 0.0047 & 0.0130 & 0.01 & 0.04 \\
2 & 0.3801 & 0.0105 & 0.0038 & 0.0110 & 0.01 & 0.03 \\
\hline
\end{tabular}

$\mathrm{K}_{2} \mathrm{O}$ Powder, Replicate 2

\begin{tabular}{crrrrrr}
\hline Material & Xbar & $\boldsymbol{s}_{\boldsymbol{x}}$ & $\boldsymbol{s}_{\boldsymbol{r}}$ & $\boldsymbol{s}_{\boldsymbol{R}}$ & $\boldsymbol{r}$ & $\boldsymbol{R}$ \\
\hline 1 & 0.6823 & 0.0125 & 0.0047 & 0.0132 & 0.01 & 0.04 \\
2 & 0.3801 & 0.0103 & 0.0036 & 0.0108 & 0.01 & 0.03 \\
\hline
\end{tabular}

\section{K20}

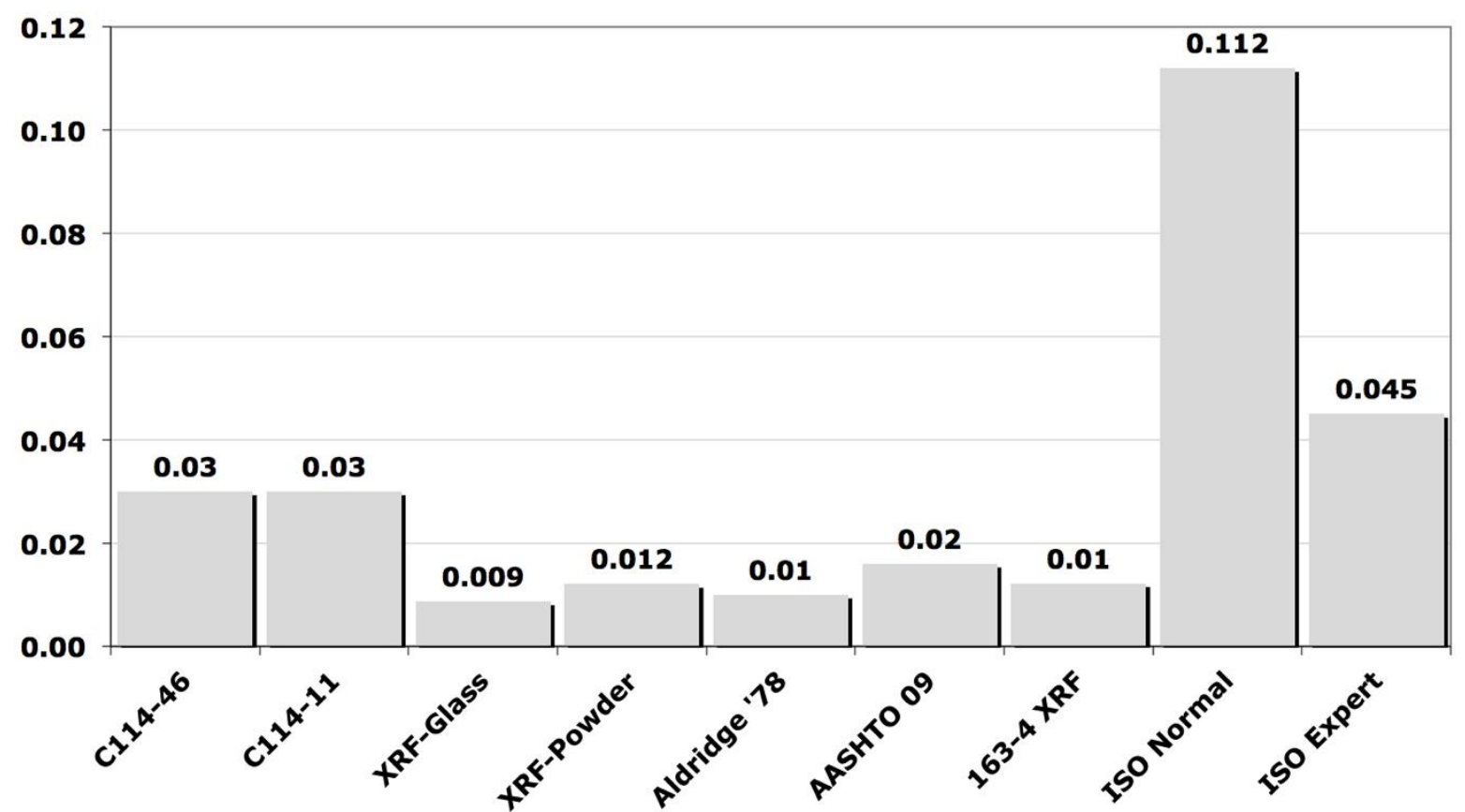

Figure $22 \mathrm{~K}_{2} \mathrm{O}$ precision statistics by method with bar chart comparing results to current and past ASTM C114 limits and previous studies on chemical analysis precision as 1-sigma, between lab $\left(S_{R}\right)$. 
$\mathrm{TiO}_{2}$

TiO2
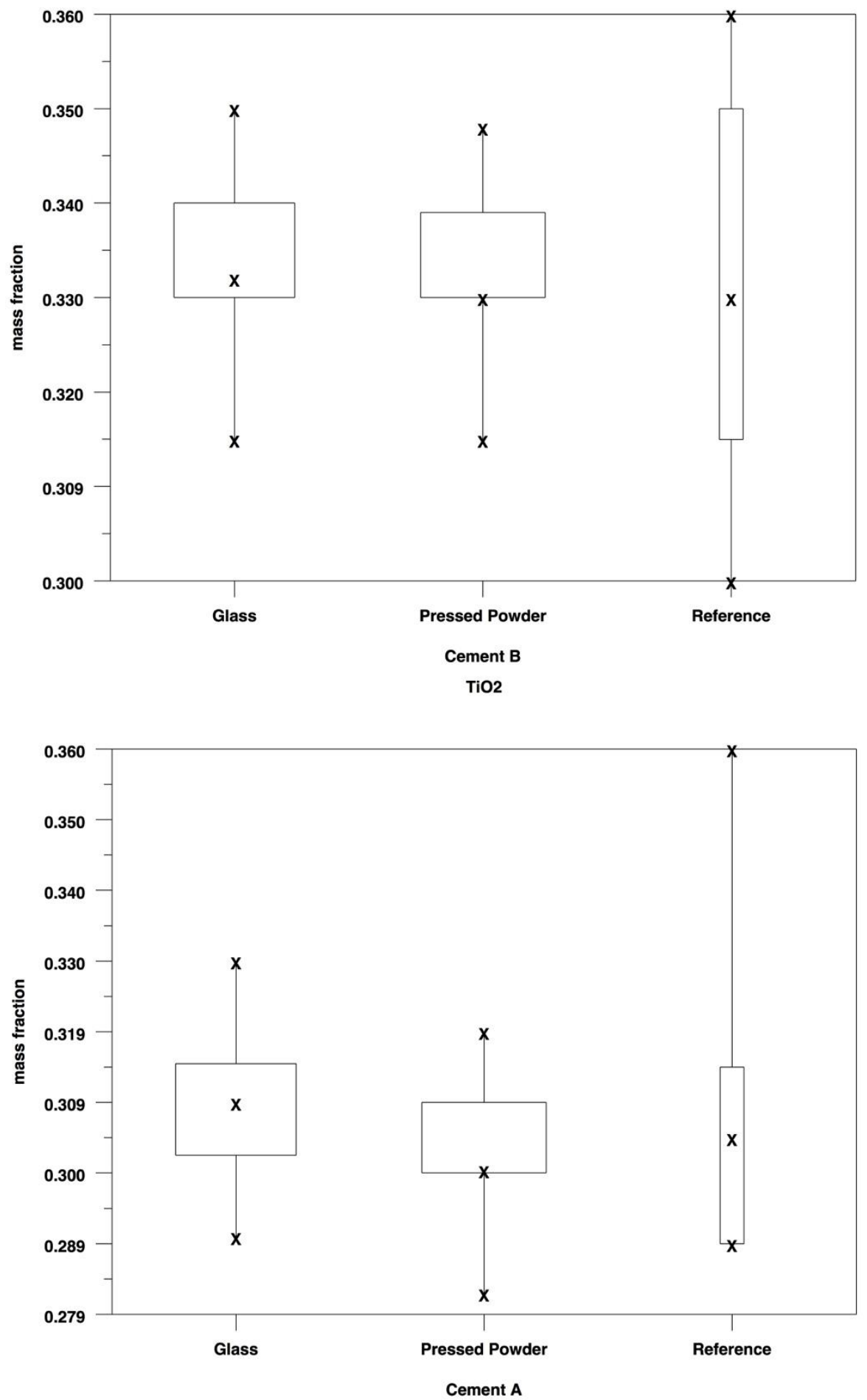

Figure 23 Box plots for $\mathrm{TiO}_{2} \mathrm{XRF}$ glass and powder, and reference methods 
$\mathrm{TiO}_{2}$ XRF, Cements A B, Glass, Replicate 1, Material: 1

\begin{tabular}{crrrrr}
\hline $\begin{array}{c}\text { Laboratory } \\
\text { Number }\end{array}$ & $\begin{array}{r}\text { Cell } \\
\text { Mean }\end{array}$ & $\begin{array}{r}\text { Cell } \\
\text { SD }\end{array}$ & $\boldsymbol{d}$ & $\boldsymbol{h}$ & $\boldsymbol{k}$ \\
\hline 1 & 0.3200 & 0.0000 & 0.0105 & 1.58 & 0.00 \\
2 & 0.3097 & 0.0042 & 0.0002 & 0.03 & 1.05 \\
3 & 0.3083 & 0.0041 & -0.0011 & -0.17 & 1.02 \\
4 & 0.3083 & 0.0018 & -0.0011 & -0.17 & 0.44 \\
5 & 0.3200 & 0.0000 & 0.0105 & 1.58 & 0.00 \\
6 & 0.3023 & 0.0068 & -0.0071 & -1.07 & 1.70 \\
7 & 0.3185 & 0.0029 & 0.0090 & 1.36 & 0.72 \\
8 & 0.3050 & 0.0055 & -0.0045 & -0.67 & 1.36 \\
9 & 0.3120 & 0.0010 & 0.0026 & 0.39 & 0.25 \\
10 & 0.3167 & 0.0052 & 0.0072 & 1.08 & 1.28 \\
11 & 0.3055 & 0.0016 & -0.0040 & -0.59 & 0.41 \\
12 & 0.3067 & 0.0052 & -0.0028 & -0.42 & 1.28 \\
13 & 0.3160 & 0.0033 & 0.0065 & 0.98 & 0.82 \\
14 & 0.3033 & 0.0052 & -0.0061 & -0.92 & 1.28 \\
15 & 0.2983 & 0.0021 & -0.0111 & -1.67 & 0.51 \\
16 & 0.3033 & 0.0052 & -0.0061 & -0.92 & 1.28 \\
17 & 0.3067 & 0.0052 & -0.0028 & -0.42 & 1.28 \\
\hline
\end{tabular}

Average of cell averages $\quad=\quad 0.30945$

Standard Deviation of cell averages $=0.00666$

Repeatability Standard Deviation $=0.00402$

Reproducibility Standard Deviation $=0.00760$

$\mathrm{h}, \mathrm{k}$ Critical Values $\quad=\quad 2.51000,1.78000$ 
$\mathrm{TiO}_{2}$ XRF, Cements A B, Glass, Replicate 1, Material: 2

\begin{tabular}{crrrrr}
\hline $\begin{array}{c}\text { Laboratory } \\
\text { Number }\end{array}$ & $\begin{array}{r}\text { Cell } \\
\text { Mean }\end{array}$ & $\begin{array}{r}\text { Cell } \\
\text { SD }\end{array}$ & $\boldsymbol{d}$ & $\boldsymbol{h}$ & $\boldsymbol{k}$ \\
\hline 1 & 0.3433 & 0.0052 & 0.0094 & 1.43 & 1.16 \\
2 & 0.3353 & 0.0033 & 0.0014 & 0.21 & 0.74 \\
3 & 0.3350 & 0.0055 & 0.0010 & 0.16 & 1.23 \\
4 & 0.3330 & 0.0014 & -0.0010 & -0.15 & 0.32 \\
5 & 0.3433 & 0.0052 & 0.0094 & 1.43 & 1.16 \\
6 & 0.3278 & 0.0038 & -0.0061 & -0.94 & 0.86 \\
7 & 0.3413 & 0.0041 & 0.0074 & 1.12 & 0.93 \\
8 & 0.3267 & 0.0052 & -0.0073 & -1.12 & 1.16 \\
9 & 0.3422 & 0.0006 & 0.0082 & 1.25 & 0.13 \\
10 & 0.3400 & 0.0000 & 0.0060 & 0.92 & 0.00 \\
11 & 0.3312 & 0.0013 & -0.0028 & -0.43 & 0.30 \\
12 & 0.3333 & 0.0052 & -0.0006 & -0.10 & 1.16 \\
13 & 0.3382 & 0.0046 & 0.0042 & 0.64 & 1.03 \\
14 & 0.3283 & 0.0041 & -0.0056 & -0.86 & 0.92 \\
15 & 0.3237 & 0.0067 & -0.0103 & -1.57 & 1.50 \\
16 & 0.3250 & 0.0055 & -0.0090 & -1.37 & 1.23 \\
17 & 0.3300 & 0.0063 & -0.0040 & -0.61 & 1.42 \\
\hline
\end{tabular}

Average of cell averages $\quad=\quad 0.33398$

Standard Deviation of cell averages $=0.00656$

Repeatability Standard Deviation $=0.00444$

Reproducibility Standard Deviation $=0.00771$

$\mathrm{h}, \mathrm{k}$ Critical Values $\quad=\quad 2.51000,1.78000$ 
$\mathrm{TiO}_{2}$ XRF, Cements A B, Glass, Replicate 2, Material: 1

\begin{tabular}{crrrrr}
\hline $\begin{array}{c}\text { Laboratory } \\
\text { Number }\end{array}$ & $\begin{array}{r}\text { Cell } \\
\text { Mean }\end{array}$ & $\begin{array}{r}\text { Cell } \\
\text { SD }\end{array}$ & $\boldsymbol{d}$ & $\boldsymbol{h}$ & $\boldsymbol{k}$ \\
\hline 1 & 0.3200 & 0.0000 & 0.0106 & 1.59 & 0.00 \\
2 & 0.3097 & 0.0066 & 0.0003 & 0.04 & 1.53 \\
3 & 0.3083 & 0.0041 & -0.0010 & -0.16 & 0.94 \\
4 & 0.3070 & 0.0009 & -0.0024 & -0.35 & 0.21 \\
5 & 0.3217 & 0.0041 & 0.0123 & 1.84 & 0.94 \\
6 & 0.3033 & 0.0066 & -0.0060 & -0.90 & 1.52 \\
7 & 0.3190 & 0.0049 & 0.0096 & 1.44 & 1.12 \\
8 & 0.3000 & 0.0000 & -0.0094 & -1.40 & 0.00 \\
9 & 0.3120 & 0.0014 & 0.0026 & 0.39 & 0.32 \\
10 & 0.3133 & 0.0052 & 0.0040 & 0.59 & 1.19 \\
11 & 0.3048 & 0.0012 & -0.0045 & -0.68 & 0.27 \\
12 & 0.3050 & 0.0055 & -0.0044 & -0.65 & 1.27 \\
13 & 0.3168 & 0.0025 & 0.0075 & 1.12 & 0.57 \\
14 & 0.3033 & 0.0052 & -0.0060 & -0.90 & 1.19 \\
15 & 0.3033 & 0.0061 & -0.0060 & -0.90 & 1.41 \\
16 & 0.3033 & 0.0052 & -0.0060 & -0.90 & 1.19 \\
17 & 0.3083 & 0.0041 & -0.0010 & -0.16 & 0.94 \\
\hline
\end{tabular}

Average of cell averages $\quad=\quad 0.30937$

Standard Deviation of cell averages $=0.00669$

Repeatability Standard Deviation $=0.00432$

Reproducibility Standard Deviation $=0.00777$

$\mathrm{h}, \mathrm{k}$ Critical Values $\quad=\quad 2.51000,1.78000$ 
$\mathrm{TiO}_{2}$ XRF, Cements A B, Glass, Replicate 2, Material: 2

\begin{tabular}{crrrrr}
\hline $\begin{array}{c}\text { Laboratory } \\
\text { Number }\end{array}$ & $\begin{array}{r}\text { Cell } \\
\text { Mean }\end{array}$ & $\begin{array}{r}\text { Cell } \\
\text { SD }\end{array}$ & $\boldsymbol{d}$ & $\boldsymbol{h}$ & $\boldsymbol{k}$ \\
\hline 1 & 0.3433 & 0.0052 & 0.0101 & 1.42 & 1.18 \\
2 & 0.3350 & 0.0053 & 0.0017 & 0.25 & 1.22 \\
3 & 0.3333 & 0.0052 & 0.0001 & 0.01 & 1.18 \\
4 & 0.3323 & 0.0018 & -0.0009 & -0.13 & 0.40 \\
5 & 0.3433 & 0.0052 & 0.0101 & 1.42 & 1.18 \\
6 & 0.3263 & 0.0055 & -0.0069 & -0.98 & 1.26 \\
7 & 0.3427 & 0.0040 & 0.0094 & 1.33 & 0.92 \\
8 & 0.3283 & 0.0041 & -0.0049 & -0.70 & 0.94 \\
9 & 0.3430 & 0.0005 & 0.0097 & 1.37 & 0.12 \\
10 & 0.3400 & 0.0000 & 0.0067 & 0.95 & 0.00 \\
11 & 0.3305 & 0.0019 & -0.0028 & -0.39 & 0.43 \\
12 & 0.3267 & 0.0052 & -0.0066 & -0.93 & 1.18 \\
13 & 0.3365 & 0.0038 & 0.0032 & 0.46 & 0.87 \\
14 & 0.3283 & 0.0041 & -0.0049 & -0.70 & 0.94 \\
15 & 0.3225 & 0.0064 & -0.0108 & -1.52 & 1.46 \\
16 & 0.3250 & 0.0055 & -0.0083 & -1.17 & 1.26 \\
17 & 0.3283 & 0.0041 & -0.0049 & -0.70 & 0.94 \\
\hline
\end{tabular}

Average of cell averages $\quad=0.33326$

Standard Deviation of cell averages $=0.00708$

Repeatability Standard Deviation $=0.00436$

Reproducibility Standard Deviation $=0.00812$

$\mathrm{h}, \mathrm{k}$ Critical Values $\quad=\quad 2.51000,1.78000$ 
$\mathrm{TiO}_{2}$ XRF, Cements A B, Powder, Replicate 1, Material: 1

\begin{tabular}{crrrrr}
\hline $\begin{array}{c}\text { Laboratory } \\
\text { Number }\end{array}$ & $\begin{array}{r}\text { Cell } \\
\text { Mean }\end{array}$ & $\begin{array}{r}\text { Cell } \\
\text { SD }\end{array}$ & $\boldsymbol{d}$ & $\boldsymbol{h}$ & $\boldsymbol{k}$ \\
\hline 1 & 0.2907 & 0.0014 & -0.0112 & -1.59 & 0.47 \\
2 & 0.3025 & 0.0019 & 0.0006 & 0.09 & 0.65 \\
3 & 0.3000 & 0.0000 & -0.0019 & -0.26 & 0.00 \\
4 & 0.3127 & 0.0024 & 0.0108 & 1.53 & 0.84 \\
5 & 0.3022 & 0.0036 & 0.0003 & 0.04 & 1.25 \\
6 & 0.3000 & 0.0000 & -0.0019 & -0.26 & 0.00 \\
7 & 0.2873 & 0.0041 & -0.0145 & -2.06 & 1.43 \\
8 & 0.2933 & 0.0052 & -0.0085 & -1.21 & 1.79 \\
9 & 0.3059 & 0.0010 & 0.0040 & 0.57 & 0.35 \\
10 & 0.3067 & 0.0041 & 0.0048 & 0.68 & 1.42 \\
11 & 0.2963 & 0.0035 & -0.0056 & -0.79 & 1.20 \\
12 & 0.2983 & 0.0041 & -0.0035 & -0.50 & 1.42 \\
13 & 0.3100 & 0.0000 & 0.0081 & 1.15 & 0.00 \\
14 & 0.3000 & 0.0000 & -0.0019 & -0.26 & 0.00 \\
15 & 0.3115 & 0.0022 & 0.0096 & 1.36 & 0.75 \\
16 & 0.3033 & 0.0052 & 0.0015 & 0.21 & 1.79 \\
17 & 0.3100 & 0.0000 & 0.0081 & 1.15 & 0.00 \\
18 & 0.3030 & 0.0020 & 0.0011 & 0.16 & 0.68 \\
\hline
\end{tabular}

Average of cell averages $\quad=\quad 0.30187$

Standard Deviation of cell averages $=0.00706$

Repeatability Standard Deviation $=0.00288$

Reproducibility Standard Deviation $=0.00754$

$\mathrm{h}, \mathrm{k}$ Critical Values $\quad=\quad 2.51000,1.78000$ 
$\mathrm{TiO}_{2}$ XRF, Cements A B, Powder, Replicate 1, Material: 2

\begin{tabular}{crrrrr}
\hline $\begin{array}{c}\text { Laboratory } \\
\text { Number }\end{array}$ & $\begin{array}{r}\text { Cell } \\
\text { Mean }\end{array}$ & $\begin{array}{r}\text { Cell } \\
\text { SD }\end{array}$ & $\boldsymbol{d}$ & $\boldsymbol{h}$ & $\boldsymbol{k}$ \\
\hline 1 & 0.3210 & 0.0029 & -0.0112 & -1.67 & 1.01 \\
2 & 0.3347 & 0.0008 & 0.0025 & 0.37 & 0.28 \\
3 & 0.3300 & 0.0000 & -0.0022 & -0.32 & 0.00 \\
4 & 0.3417 & 0.0029 & 0.0095 & 1.42 & 1.03 \\
5 & 0.3370 & 0.0040 & 0.0048 & 0.72 & 1.39 \\
6 & 0.3317 & 0.0041 & -0.0005 & -0.08 & 1.42 \\
7 & 0.3317 & 0.0018 & -0.0005 & -0.08 & 0.61 \\
8 & 0.3217 & 0.0041 & -0.0105 & -1.57 & 1.42 \\
9 & 0.3414 & 0.0004 & 0.0092 & 1.38 & 0.14 \\
10 & 0.3388 & 0.0012 & 0.0067 & 1.00 & 0.41 \\
11 & 0.3222 & 0.0023 & -0.0100 & -1.49 & 0.79 \\
12 & 0.3250 & 0.0055 & -0.0072 & -1.07 & 1.91 \\
13 & 0.3383 & 0.0041 & 0.0062 & 0.92 & 1.42 \\
14 & 0.3317 & 0.0041 & -0.0005 & -0.08 & 1.42 \\
15 & 0.3407 & 0.0019 & 0.0085 & 1.27 & 0.65 \\
16 & 0.3300 & 0.0000 & -0.0022 & -0.32 & 0.00 \\
17 & 0.3300 & 0.0000 & -0.0022 & -0.32 & 0.00 \\
18 & 0.3316 & 0.0022 & -0.0005 & -0.08 & 0.76 \\
\hline
\end{tabular}

Average of cell averages $\quad=0.33217$

Standard Deviation of cell averages $=0.00669$

Repeatability Standard Deviation $=0.00287$

Reproducibility Standard Deviation $=0.00718$

$\mathrm{h}, \mathrm{k}$ Critical Values $\quad=\quad 2.51000,1.78000$ 
$\mathrm{TiO}_{2}$ XRF, Cements A B, Powder, Replicate 2, Material: 1

\begin{tabular}{crrrrr}
\hline $\begin{array}{c}\text { Laboratory } \\
\text { Number }\end{array}$ & $\begin{array}{r}\text { Cell } \\
\text { Mean }\end{array}$ & $\begin{array}{r}\text { Cell } \\
\text { SD }\end{array}$ & $\boldsymbol{d}$ & $\boldsymbol{h}$ & $\boldsymbol{k}$ \\
\hline 1 & 0.2908 & 0.0026 & -0.0117 & -1.70 & 0.77 \\
2 & 0.3030 & 0.0011 & 0.0005 & 0.07 & 0.33 \\
3 & 0.3000 & 0.0000 & -0.0025 & -0.37 & 0.00 \\
4 & 0.3117 & 0.0022 & 0.0091 & 1.33 & 0.65 \\
5 & 0.3053 & 0.0027 & 0.0028 & 0.41 & 0.82 \\
6 & 0.3000 & 0.0000 & -0.0025 & -0.37 & 0.00 \\
7 & 0.2893 & 0.0027 & -0.0132 & -1.92 & 0.82 \\
8 & 0.2950 & 0.0055 & -0.0075 & -1.10 & 1.65 \\
9 & 0.3053 & 0.0006 & 0.0028 & 0.41 & 0.17 \\
10 & 0.3087 & 0.0039 & 0.0061 & 0.89 & 1.17 \\
11 & 0.2973 & 0.0018 & -0.0052 & -0.76 & 0.54 \\
12 & 0.2983 & 0.0075 & -0.0042 & -0.61 & 2.27 \\
13 & 0.3117 & 0.0041 & 0.0091 & 1.33 & 1.23 \\
14 & 0.3033 & 0.0052 & 0.0008 & 0.11 & 1.55 \\
15 & 0.3125 & 0.0014 & 0.0100 & 1.45 & 0.42 \\
16 & 0.3017 & 0.0041 & -0.0009 & -0.13 & 1.23 \\
17 & 0.3100 & 0.0000 & 0.0075 & 1.09 & 0.00 \\
18 & 0.3018 & 0.0020 & -0.0008 & -0.11 & 0.61 \\
\hline
\end{tabular}

Average of cell averages $\quad=\quad 0.30254$

Standard Deviation of cell averages $=0.00687$

Repeatability Standard Deviation $=0.00332$

Reproducibility Standard Deviation $=0.00751$

$\mathrm{h}, \mathrm{k}$ Critical Values $\quad=\quad 2.53000,1.78000$ 
$\mathrm{TiO}_{2}$ XRF, Cements A B, Powder, Replicate 2, Material: 2

\begin{tabular}{crrrrr}
\hline $\begin{array}{c}\text { Laboratory } \\
\text { Number }\end{array}$ & $\begin{array}{r}\text { Cell } \\
\text { Mean }\end{array}$ & $\begin{array}{r}\text { Cell } \\
\text { SD }\end{array}$ & $\boldsymbol{d}$ & $\boldsymbol{h}$ & $\boldsymbol{k}$ \\
\hline 1 & 0.3202 & 0.0029 & -0.0121 & -1.70 & 0.87 \\
2 & 0.3332 & 0.0024 & 0.0009 & 0.12 & 0.73 \\
3 & 0.3300 & 0.0000 & -0.0023 & -0.32 & 0.00 \\
4 & 0.3413 & 0.0050 & 0.0091 & 1.27 & 1.52 \\
5 & 0.3378 & 0.0043 & 0.0056 & 0.78 & 1.30 \\
6 & 0.3283 & 0.0041 & -0.0039 & -0.55 & 1.25 \\
7 & 0.3305 & 0.0028 & -0.0018 & -0.25 & 0.86 \\
8 & 0.3250 & 0.0055 & -0.0073 & -1.02 & 1.67 \\
9 & 0.3413 & 0.0005 & 0.0091 & 1.27 & 0.16 \\
10 & 0.3405 & 0.0023 & 0.0082 & 1.15 & 0.69 \\
11 & 0.3223 & 0.0025 & -0.0100 & -1.40 & 0.75 \\
12 & 0.3250 & 0.0055 & -0.0073 & -1.02 & 1.67 \\
13 & 0.3400 & 0.0000 & 0.0077 & 1.08 & 0.00 \\
14 & 0.3317 & 0.0041 & -0.0006 & -0.09 & 1.25 \\
15 & 0.3442 & 0.0016 & 0.0119 & 1.67 & 0.49 \\
16 & 0.3300 & 0.0000 & -0.0023 & -0.32 & 0.00 \\
17 & 0.3283 & 0.0041 & -0.0039 & -0.55 & 1.25 \\
18 & 0.3313 & 0.0021 & -0.0009 & -0.13 & 0.64 \\
\hline
\end{tabular}

Average of cell averages $\quad=0.33228$

Standard Deviation of cell averages $=0.00712$

Repeatability Standard Deviation $=0.00328$

Reproducibility Standard Deviation $=0.00773$

$\mathrm{h}, \mathrm{k}$ Critical Values $\quad=\quad 2.53000,1.78000$ 
Table 11 Precision Statistics $\mathrm{TiO}_{2}$ by XRF glass and powder preparations for replicates 1 and 2.

$\mathrm{TiO}_{2}$ Glass, Replicate 1

\begin{tabular}{crrrrrr}
\hline Material & Xbar & $\boldsymbol{s}_{\boldsymbol{x}}$ & $\boldsymbol{s}_{\boldsymbol{r}}$ & $\boldsymbol{s}_{\boldsymbol{R}}$ & $\boldsymbol{r}$ & $\boldsymbol{R}$ \\
\hline 1 & 0.3095 & 0.0067 & 0.0040 & 0.0076 & 0.01 & 0.02 \\
2 & 0.3340 & 0.0066 & 0.0044 & 0.0077 & 0.01 & 0.02 \\
\hline
\end{tabular}

$\mathrm{TiO}_{2}$ Glass, Replicate 2

\begin{tabular}{crrrrrr}
\hline Material & Xbar & $\boldsymbol{s}_{\boldsymbol{x}}$ & $\boldsymbol{s}_{\boldsymbol{r}}$ & $\boldsymbol{s}_{\boldsymbol{R}}$ & $\boldsymbol{r}$ & $\boldsymbol{R}$ \\
\hline 1 & 0.3094 & 0.0067 & 0.0043 & 0.0078 & 0.01 & 0.02 \\
2 & 0.3333 & 0.0071 & 0.0044 & 0.0081 & 0.01 & 0.02 \\
\hline
\end{tabular}

$\mathrm{TiO}_{2}$ Powder, Replicate 1

\begin{tabular}{crrrrrr}
\hline Material & Xbar & $\boldsymbol{s}_{\boldsymbol{x}}$ & $\boldsymbol{s}_{\boldsymbol{r}}$ & $\boldsymbol{s}_{\boldsymbol{R}}$ & $\boldsymbol{r}$ & $\boldsymbol{R}$ \\
\hline 1 & 0.3019 & 0.0071 & 0.0029 & 0.0075 & 0.01 & 0.02 \\
2 & 0.3322 & 0.0067 & 0.0029 & 0.0072 & 0.01 & 0.02 \\
\hline
\end{tabular}

$\mathrm{TiO}_{2}$ Powder, Replicate 2

\begin{tabular}{crrrrrr}
\hline Material & Xbar & $\boldsymbol{s}_{\boldsymbol{x}}$ & $\boldsymbol{s}_{\boldsymbol{r}}$ & $\boldsymbol{s}_{\boldsymbol{R}}$ & $\boldsymbol{r}$ & $\boldsymbol{R}$ \\
\hline 1 & 0.3025 & 0.0069 & 0.0033 & 0.0075 & 0.01 & 0.02 \\
2 & 0.3323 & 0.0071 & 0.0033 & 0.0077 & 0.01 & 0.02 \\
\hline
\end{tabular}

TiO2

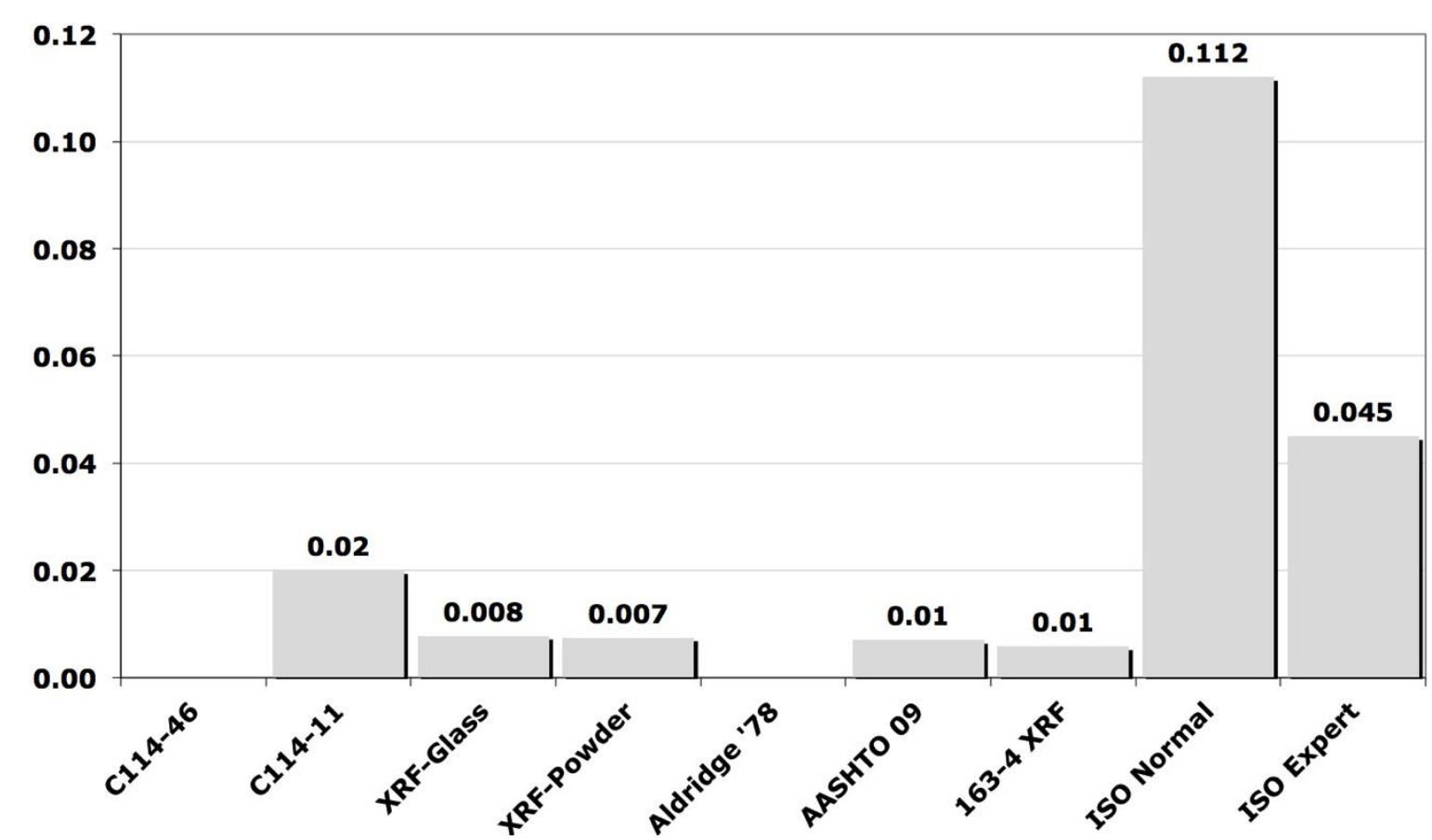

Figure $24 \mathrm{TiO}_{2}$ precision statistics by method with bar chart comparing results to current and past ASTM C114 limits and previous studies on chemical analysis precision as 1 -sigma, between lab $\left(\mathrm{S}_{\mathrm{R}}\right)$. 
$\mathrm{P}_{2} \mathrm{O}_{5}$

P2O5
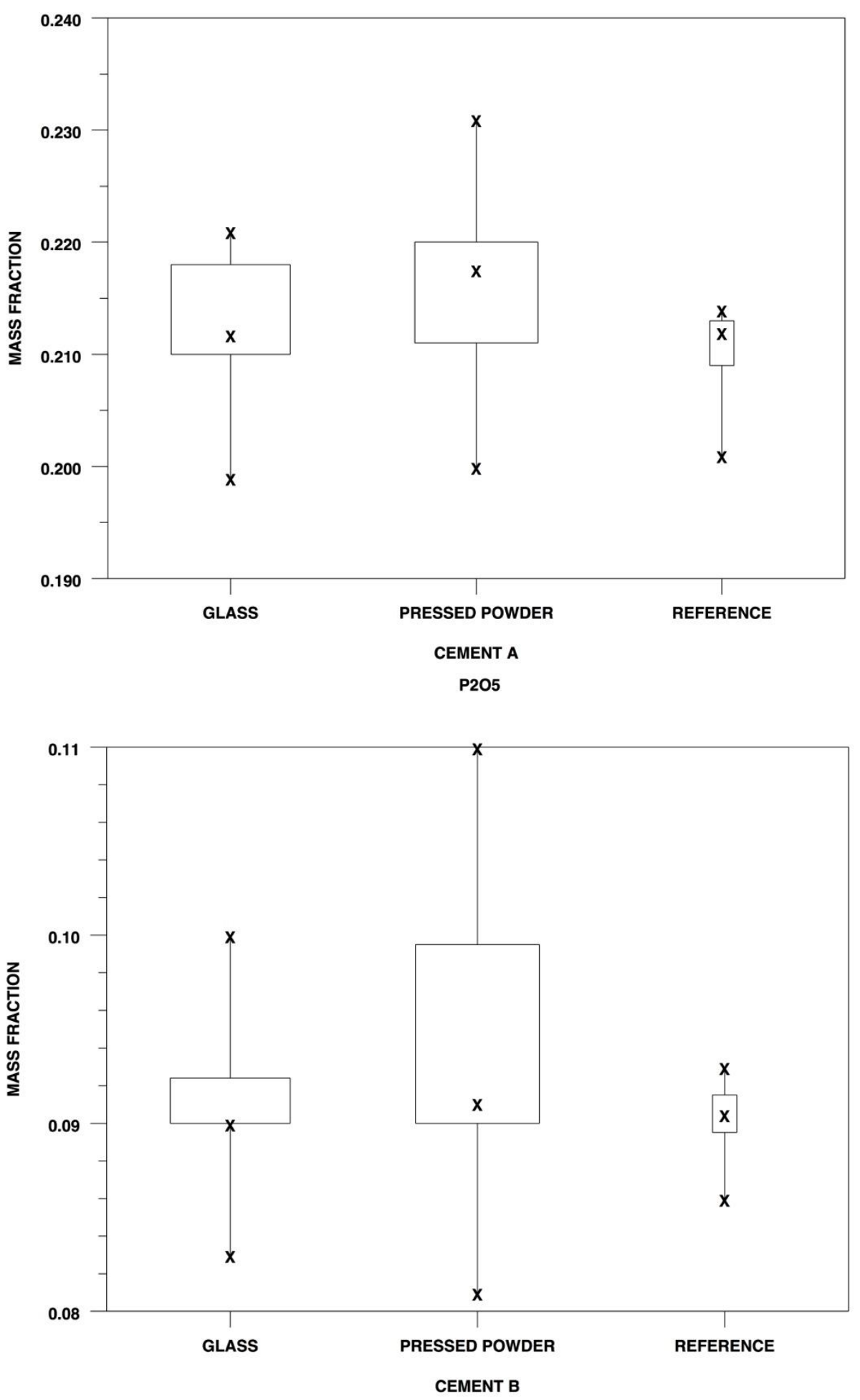

Figure 25 Box plots for $\mathrm{P}_{2} \mathrm{O}_{5}$ XRF glass and powder, and reference methods. 
$\mathrm{P}_{2} \mathrm{O}_{5}$ XRF, Cements A B, Glass, Replicate 1, Material: 2

\begin{tabular}{crrrrr}
\hline $\begin{array}{c}\text { Laboratory } \\
\text { Number }\end{array}$ & $\begin{array}{r}\text { Cell } \\
\text { Mean }\end{array}$ & $\begin{array}{r}\text { Cell } \\
\text { SD }\end{array}$ & $\boldsymbol{d}$ & $\boldsymbol{h}$ & $\boldsymbol{k}$ \\
\hline 1 & 0.2058 & 0.0083 & -0.0068 & -1.65 & 2.04 \\
2 & 0.2108 & 0.0007 & -0.0018 & -0.44 & 0.18 \\
3 & 0.2183 & 0.0041 & 0.0057 & 1.38 & 1.00 \\
4 & 0.2160 & 0.0019 & 0.0034 & 0.81 & 0.46 \\
5 & 0.2175 & 0.0026 & 0.0049 & 1.18 & 0.63 \\
6 & 0.2117 & 0.0041 & -0.0010 & -0.24 & 1.00 \\
7 & 0.2133 & 0.0022 & 0.0007 & 0.17 & 0.53 \\
8 & 0.2150 & 0.0055 & 0.0024 & 0.57 & 1.34 \\
9 & 0.2093 & 0.0014 & -0.0033 & -0.80 & 0.33 \\
10 & 0.2083 & 0.0075 & -0.0043 & -1.05 & 1.84 \\
11 & 0.2200 & 0.0000 & 0.0074 & 1.79 & 0.00 \\
12 & 0.2132 & 0.0050 & 0.0005 & 0.13 & 1.23 \\
13 & 0.2067 & 0.0052 & -0.0060 & -1.45 & 1.26 \\
14 & 0.2145 & 0.0002 & 0.0019 & 0.45 & 0.05 \\
15 & 0.2100 & 0.0000 & -0.0026 & -0.64 & 0.00 \\
16 & 0.2118 & 0.0029 & -0.0008 & -0.20 & 0.72 \\
\hline
\end{tabular}

Average of cell averages $\quad=\quad 0.21265$

Standard Deviation of cell averages $=0.00412$

Repeatability Standard Deviation $=0.00409$

Reproducibility Standard Deviation $=0.00556$

$\mathrm{h}, \mathrm{k}$ Critical Values $\quad=\quad 2.49000,1.77000$ 
$\mathrm{P}_{2} \mathrm{O}_{5}$ XRF, Cements A B, Glass, Replicate 1, Material: 2

\begin{tabular}{crrrrr}
\hline $\begin{array}{c}\text { Laboratory } \\
\text { Number }\end{array}$ & $\begin{array}{r}\text { Cell } \\
\text { Mean }\end{array}$ & $\begin{array}{r}\text { Cell } \\
\text { SD }\end{array}$ & $\boldsymbol{d}$ & $\boldsymbol{h}$ & $\boldsymbol{k}$ \\
\hline 1 & 0.0930 & 0.0037 & 0.0018 & 1.36 & 1.46 \\
2 & 0.0923 & 0.0011 & 0.0012 & 0.86 & 0.42 \\
3 & 0.0900 & 0.0000 & -0.0012 & -0.86 & 0.00 \\
4 & 0.0920 & 0.0017 & 0.0008 & 0.62 & 0.66 \\
5 & 0.0930 & 0.0020 & 0.0018 & 1.36 & 0.79 \\
6 & 0.0900 & 0.0000 & -0.0012 & -0.86 & 0.00 \\
7 & 0.0925 & 0.0015 & 0.0013 & 0.99 & 0.60 \\
8 & 0.0900 & 0.0000 & -0.0012 & -0.86 & 0.00 \\
9 & 0.0900 & 0.0006 & -0.0012 & -0.86 & 0.25 \\
10 & 0.0900 & 0.0000 & -0.0012 & -0.86 & 0.00 \\
11 & 0.0917 & 0.0041 & 0.0005 & 0.37 & 1.62 \\
12 & 0.0918 & 0.0049 & 0.0007 & 0.50 & 1.95 \\
13 & 0.0917 & 0.0041 & 0.0005 & 0.37 & 1.62 \\
14 & 0.0921 & 0.0002 & 0.0009 & 0.67 & 0.08 \\
15 & 0.0900 & 0.0000 & -0.0012 & -0.86 & 0.00 \\
16 & 0.0885 & 0.0045 & -0.0027 & -1.96 & 1.77 \\
\hline
\end{tabular}

Average of cell averages $\quad=\quad 0.09116$

Standard Deviation of cell averages $=0.00136$

Repeatability Standard Deviation $=0.00252$

Reproducibility Standard Deviation $=0.00267$

$\mathrm{h}, \mathrm{k}$ Critical Values $\quad=\quad 2.49000,1.77000$ 
$\mathrm{P}_{2} \mathrm{O}_{5}$ XRF, Cements A B, Glass, Replicate 2, Material: 1

\begin{tabular}{crrrrr}
\hline $\begin{array}{c}\text { Laboratory } \\
\text { Number }\end{array}$ & $\begin{array}{r}\text { Cell } \\
\text { Mean }\end{array}$ & $\begin{array}{r}\text { Cell } \\
\text { SD }\end{array}$ & $\boldsymbol{d}$ & $\boldsymbol{h}$ & $\boldsymbol{k}$ \\
\hline 1 & 0.2120 & 0.0024 & -0.0010 & -0.23 & 0.65 \\
2 & 0.2110 & 0.0006 & -0.0020 & -0.44 & 0.16 \\
3 & 0.2167 & 0.0052 & 0.0036 & 0.78 & 1.43 \\
4 & 0.2143 & 0.0023 & 0.0013 & 0.28 & 0.65 \\
5 & 0.2173 & 0.0020 & 0.0043 & 0.93 & 0.54 \\
6 & 0.2117 & 0.0041 & -0.0014 & -0.30 & 1.13 \\
7 & 0.2142 & 0.0023 & 0.0011 & 0.24 & 0.64 \\
8 & 0.2183 & 0.0041 & 0.0053 & 1.14 & 1.13 \\
9 & 0.2095 & 0.0016 & -0.0035 & -0.77 & 0.45 \\
10 & 0.2067 & 0.0052 & -0.0064 & -1.38 & 1.43 \\
11 & 0.2200 & 0.0000 & 0.0070 & 1.50 & 0.00 \\
12 & 0.2168 & 0.0040 & 0.0038 & 0.82 & 1.11 \\
13 & 0.2017 & 0.0041 & -0.0114 & -2.46 & 1.13 \\
14 & 0.2144 & 0.0004 & 0.0013 & 0.28 & 0.11 \\
15 & 0.2133 & 0.0052 & 0.0003 & 0.06 & 1.43 \\
16 & 0.2108 & 0.0063 & -0.0022 & -0.48 & 1.75 \\
\hline
\end{tabular}

Average of cell averages $\quad=\quad 0.21304$

Standard Deviation of cell averages $=0.00463$

Repeatability Standard Deviation $=0.00362$

Reproducibility Standard Deviation $=0.00569$

$\mathrm{h}, \mathrm{k}$ Critical Values $\quad=\quad 2.49000,1.77000$ 
$\mathrm{P}_{2} \mathrm{O}_{5}$ XRF, Cements A B, Glass, Replicate 2, Material: 2

\begin{tabular}{crrrrr}
\hline $\begin{array}{c}\text { Laboratory } \\
\text { Number }\end{array}$ & $\begin{array}{r}\text { Cell } \\
\text { Mean }\end{array}$ & $\begin{array}{r}\text { Cell } \\
\text { SD }\end{array}$ & $\boldsymbol{d}$ & $\boldsymbol{h}$ & $\boldsymbol{k}$ \\
\hline 1 & 0.0947 & 0.0027 & 0.0029 & 1.86 & 1.08 \\
2 & 0.0928 & 0.0009 & 0.0011 & 0.68 & 0.35 \\
3 & 0.0900 & 0.0000 & -0.0018 & -1.12 & 0.00 \\
4 & 0.0913 & 0.0016 & -0.0004 & -0.27 & 0.65 \\
5 & 0.0925 & 0.0015 & 0.0007 & 0.47 & 0.60 \\
6 & 0.0900 & 0.0000 & -0.0018 & -1.12 & 0.00 \\
7 & 0.0933 & 0.0023 & 0.0016 & 1.01 & 0.93 \\
8 & 0.0900 & 0.0000 & -0.0018 & -1.12 & 0.00 \\
9 & 0.0903 & 0.0005 & -0.0014 & -0.91 & 0.20 \\
10 & 0.0900 & 0.0000 & -0.0018 & -1.12 & 0.00 \\
11 & 0.0933 & 0.0052 & 0.0016 & 1.01 & 2.05 \\
12 & 0.0937 & 0.0043 & 0.0019 & 1.22 & 1.69 \\
13 & 0.0917 & 0.0041 & -0.0001 & -0.06 & 1.62 \\
14 & 0.0923 & 0.0001 & 0.0005 & 0.34 & 0.05 \\
15 & 0.0900 & 0.0000 & -0.0018 & -1.12 & 0.00 \\
16 & 0.0922 & 0.0046 & 0.0004 & 0.26 & 1.83 \\
\hline
\end{tabular}

Average of cell averages $\quad=\quad 0.09176$

Standard Deviation of cell averages $=0.00157$

Repeatability Standard Deviation $=0.00252$

Reproducibility Standard Deviation $=0.00279$

$\mathrm{h}, \mathrm{k}$ Critical Values $\quad=\quad 2.49000,1.77000$ 
$\mathrm{P}_{2} \mathrm{O}_{5}$ XRF, Cements A B, Powder, Replicate 1, Material: 2

\begin{tabular}{|c|c|c|c|c|c|}
\hline $\begin{array}{l}\text { Laboratory } \\
\text { Number } \\
\end{array}$ & $\begin{array}{l}\text { Cell } \\
\text { Mean } \\
\end{array}$ & $\begin{array}{l}\text { Cell } \\
\text { SD } \\
\end{array}$ & $d$ & $\boldsymbol{h}$ & $\boldsymbol{k}$ \\
\hline 1 & 0.2200 & 0.0000 & 0.0037 & 0.66 & 0.00 \\
\hline 2 & 0.2300 & 0.0000 & 0.0137 & 2.42 & 0.00 \\
\hline 3 & 0.2168 & 0.0012 & 0.0006 & 0.10 & 0.35 \\
\hline 4 & 0.2107 & 0.0018 & -0.0056 & -0.99 & 0.52 \\
\hline 5 & 0.2200 & 0.0000 & 0.0037 & 0.66 & 0.00 \\
\hline 6 & 0.2200 & 0.0000 & 0.0037 & 0.66 & 0.00 \\
\hline 7 & 0.2190 & 0.0021 & 0.0027 & 0.48 & 0.62 \\
\hline 8 & 0.2183 & 0.0041 & 0.0021 & 0.36 & 1.21 \\
\hline 9 & 0.2102 & 0.0015 & -0.0061 & -1.08 & 0.44 \\
\hline 10 & 0.2130 & 0.0009 & -0.0033 & -0.58 & 0.26 \\
\hline 11 & 0.2067 & 0.0052 & -0.0096 & -1.70 & 1.53 \\
\hline 12 & 0.2144 & 0.0017 & -0.0019 & -0.34 & 0.50 \\
\hline 13 & 0.2083 & 0.0041 & -0.0079 & -1.40 & 1.21 \\
\hline 14 & 0.2199 & 0.0005 & 0.0036 & 0.64 & 0.14 \\
\hline 15 & 0.2200 & 0.0000 & 0.0037 & 0.66 & 0.00 \\
\hline 16 & 0.2152 & 0.0037 & -0.0011 & -0.20 & 1.08 \\
\hline 17 & 0.2143 & 0.0103 & -0.0019 & -0.34 & 3.05 \\
\hline
\end{tabular}

Average of cell averages $\quad=\quad 0.21628$

Standard Deviation of cell averages $=0.00566$

Repeatability Standard Deviation $=0.00338$

Reproducibility Standard Deviation $=0.00645$

$\mathrm{h}, \mathrm{k}$ Critical Values $\quad=\quad 2.51000,1.78000$ 
$\mathrm{P}_{2} \mathrm{O}_{5}$ XRF, Cements A B, Powder, Replicate 1, Material: 2

\begin{tabular}{lllrrl}
\hline $\begin{array}{l}\text { Laboratory } \\
\text { Number }\end{array}$ & $\begin{array}{l}\text { Cell } \\
\text { Mean }\end{array}$ & Cell & \multicolumn{1}{l}{ SD } & \multicolumn{1}{l}{$l$} & \multicolumn{1}{l}{$l$} \\
\hline 1 & 0.0917 & 0.0041 & -0.0031 & -0.38 & 0.33 \\
2 & 0.1050 & 0.0055 & 0.0103 & 1.26 & 0.45 \\
3 & 0.0895 & 0.0019 & -0.0052 & -0.65 & 0.15 \\
4 & 0.0870 & 0.0019 & -0.0077 & -0.95 & 0.16 \\
5 & 0.1000 & 0.0000 & 0.0053 & 0.65 & 0.00 \\
6 & 0.0900 & 0.0000 & -0.0047 & -0.58 & 0.00 \\
7 & 0.0903 & 0.0012 & -0.0044 & -0.54 & 0.10 \\
8 & 0.1017 & 0.0041 & 0.0069 & 0.85 & 0.33 \\
9 & 0.0917 & 0.0016 & -0.0031 & -0.38 & 0.13 \\
10 & 0.0910 & 0.0011 & -0.0037 & -0.46 & 0.09 \\
11 & 0.0900 & 0.0000 & -0.0047 & -0.58 & 0.00 \\
12 & 0.0945 & 0.0009 & -0.0002 & -0.03 & 0.08 \\
13 & 0.0900 & 0.0000 & -0.0047 & -0.58 & 0.00 \\
14 & 0.0913 & 0.0002 & -0.0035 & -0.43 & 0.02 \\
15 & 0.1200 & 0.0490 & 0.0253 & 3.11 & 4.01 \\
16 & 0.0960 & 0.0037 & 0.0013 & 0.16 & 0.31 \\
17 & 0.0910 & 0.0073 & -0.0037 & -0.46 & 0.59 \\
\hline
\end{tabular}

Average of cell averages $\quad=\quad 0.09474$

Standard Deviation of cell averages $=0.00811$

Repeatability Standard Deviation $=0.01223$

Reproducibility Standard Deviation $=0.01380$

$\mathrm{h}, \mathrm{k}$ Critical Values $\quad=\quad 2.51000,1.78000$ 
$\mathrm{P}_{2} \mathrm{O}_{5}$ XRF, Cements A B, Powder, Replicate 2, Material: 1

\begin{tabular}{llllrl}
\hline Laboratory & Cell & Cell & & & \multicolumn{1}{l}{$h$} \\
Number & Mean & SD & \multicolumn{1}{l}{$k$} \\
\hline 1 & 0.2200 & 0.0000 & 0.0038 & 0.70 & 0.00 \\
2 & 0.2283 & 0.0041 & 0.0121 & 2.24 & 1.30 \\
3 & 0.2167 & 0.0015 & 0.0005 & 0.09 & 0.48 \\
4 & 0.2117 & 0.0022 & -0.0045 & -0.84 & 0.69 \\
5 & 0.2200 & 0.0000 & 0.0038 & 0.70 & 0.00 \\
6 & 0.2200 & 0.0000 & 0.0038 & 0.70 & 0.00 \\
7 & 0.2190 & 0.0009 & 0.0028 & 0.52 & 0.29 \\
8 & 0.2183 & 0.0041 & 0.0021 & 0.39 & 1.30 \\
9 & 0.2103 & 0.0016 & -0.0059 & -1.08 & 0.52 \\
10 & 0.2125 & 0.0016 & -0.0037 & -0.68 & 0.53 \\
11 & 0.2050 & 0.0055 & -0.0112 & -2.07 & 1.75 \\
12 & 0.2143 & 0.0022 & -0.0019 & -0.34 & 0.69 \\
13 & 0.2100 & 0.0000 & -0.0062 & -1.14 & 0.00 \\
14 & 0.2197 & 0.0004 & 0.0035 & 0.64 & 0.13 \\
15 & 0.2200 & 0.0000 & 0.0038 & 0.70 & 0.00 \\
16 & 0.2152 & 0.0041 & -0.0010 & -0.19 & 1.30 \\
17 & 0.2143 & 0.0083 & -0.0019 & -0.34 & 2.65 \\
\hline
\end{tabular}

Average of cell averages $\quad=\quad 0.21620$

Standard Deviation of cell averages $=0.00542$

Repeatability Standard Deviation $=0.00313$

Reproducibility Standard Deviation $=0.00613$

$\mathrm{h}, \mathrm{k}$ Critical Values $\quad=\quad 2.51000,1.78000$ 
$\mathrm{P}_{2} \mathrm{O}_{5}$ XRF, Cements A B, Powder, Replicate 2, Material: 2

\begin{tabular}{lllrrl}
\hline Laboratory & Cell & Cell & & \\
Number & Mean & SD & \multicolumn{1}{l}{$l$} & \multicolumn{1}{l}{$h$} \\
\hline 1 & 0.0933 & 0.0052 & 0.0000 & 0.01 & 1.83 \\
2 & 0.0983 & 0.0041 & 0.0050 & 1.16 & 1.44 \\
3 & 0.0895 & 0.0016 & -0.0038 & -0.88 & 0.58 \\
4 & 0.0870 & 0.0018 & -0.0063 & -1.45 & 0.63 \\
5 & 0.1000 & 0.0000 & 0.0067 & 1.55 & 0.00 \\
6 & 0.0900 & 0.0000 & -0.0033 & -0.76 & 0.00 \\
7 & 0.0915 & 0.0008 & -0.0018 & -0.42 & 0.30 \\
8 & 0.1017 & 0.0041 & 0.0084 & 1.93 & 1.44 \\
9 & 0.0923 & 0.0021 & -0.0010 & -0.22 & 0.73 \\
10 & 0.0905 & 0.0015 & -0.0028 & -0.65 & 0.54 \\
11 & 0.0900 & 0.0000 & -0.0033 & -0.76 & 0.00 \\
12 & 0.0945 & 0.0006 & 0.0012 & 0.28 & 0.22 \\
13 & 0.0905 & 0.0012 & -0.0028 & -0.65 & 0.43 \\
14 & 0.0911 & 0.0002 & -0.0022 & -0.50 & 0.08 \\
15 & 0.1000 & 0.0000 & 0.0067 & 1.55 & 0.00 \\
16 & 0.0955 & 0.0039 & 0.0022 & 0.51 & 1.39 \\
17 & 0.0903 & 0.0067 & -0.0030 & -0.68 & 2.38 \\
\hline
\end{tabular}

Average of cell averages $\quad=\quad 0.09330$

Standard Deviation of cell averages $=0.00433$

Repeatability Standard Deviation $=0.00283$

Reproducibility Standard Deviation $=0.00505$

$\mathrm{h}, \mathrm{k}$ Critical Values $\quad=\quad 2.51000,1.78000$ 
Table 12 Precision Statistics $\mathrm{P}_{2} \mathrm{O}_{5}$ by XRF glass and powder preparations for replicates 1 and 2.

$\mathrm{P}_{2} \mathrm{O}_{5}$ Glass, Rep. 1

\begin{tabular}{lllllll}
\hline Material & Xbar & $s_{x}$ & $s_{r}$ & $s_{R}$ & $r$ & $R$ \\
\hline 1 & 0.2126 & 0.0041 & 0.0041 & 0.0056 & 0.01 & 0.02 \\
2 & 0.0912 & 0.0014 & 0.0025 & 0.0027 & 0.01 & 0.01 \\
\hline
\end{tabular}

$\mathrm{P}_{2} \mathrm{O}_{5}$ Glass, rep. 2

\begin{tabular}{lllllll}
\hline Material & Xbar & $s_{x}$ & $s_{r}$ & $s_{R}$ & $r$ & $R$ \\
\hline 1 & 0.2130 & 0.0046 & 0.0036 & 0.0057 & 0.01 & 0.02 \\
2 & 0.0918 & 0.0016 & 0.0025 & 0.0028 & 0.01 & 0.01 \\
\hline
\end{tabular}

$\mathrm{P}_{2} \mathrm{O}_{5}$ Powder, rep. 1

\begin{tabular}{lllllll}
\hline Material & Xbar & $s_{x}$ & $s_{r}$ & $s_{R}$ & $r$ & $R$ \\
\hline 1 & 0.2163 & 0.0057 & 0.0034 & 0.0064 & 0.01 & 0.02 \\
2 & 0.0947 & 0.0081 & 0.0122 & 0.0138 & 0.03 & 0.04 \\
\hline
\end{tabular}

$\mathrm{P}_{2} \mathrm{O}_{5}$ Powder, rep. 2

\begin{tabular}{lllllll}
\hline Material & Xbar & $s_{x}$ & $s_{r}$ & $s_{R}$ & $r$ & $R$ \\
\hline 1 & 0.2162 & 0.0054 & 0.0031 & 0.0061 & 0.01 & 0.02 \\
2 & 0.0933 & 0.0043 & 0.0028 & 0.0050 & 0.01 & 0.01 \\
\hline
\end{tabular}

\section{P205}

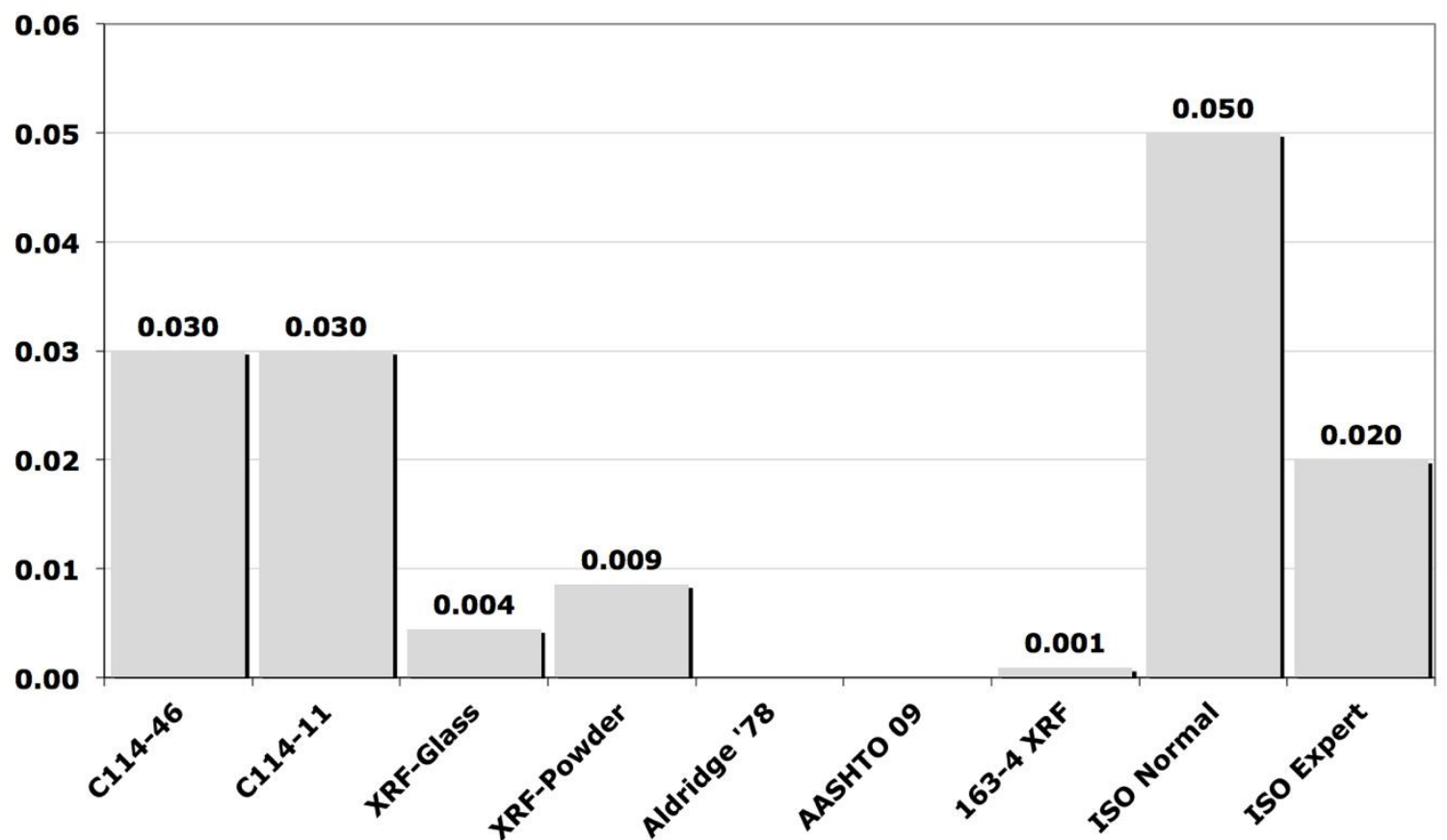

Figure $26 \mathrm{P}_{2} \mathrm{O}_{5}$ precision statistics by method with bar chart comparing results to current and past ASTM C114 limits and previous studies on chemical analysis precision as 1-sigma, between lab $\left(\mathrm{S}_{\mathrm{R}}\right)$. 
$\mathrm{Cl}$

CI

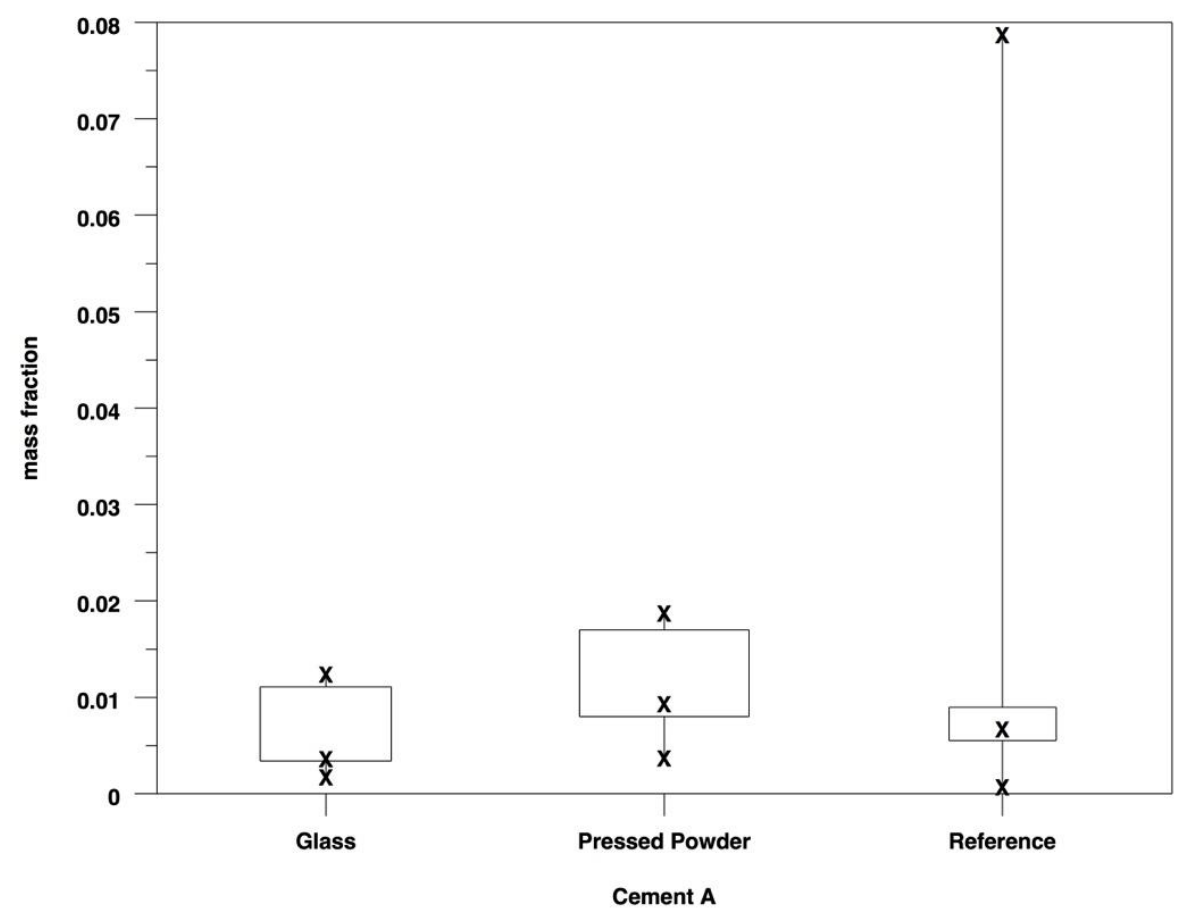

CI

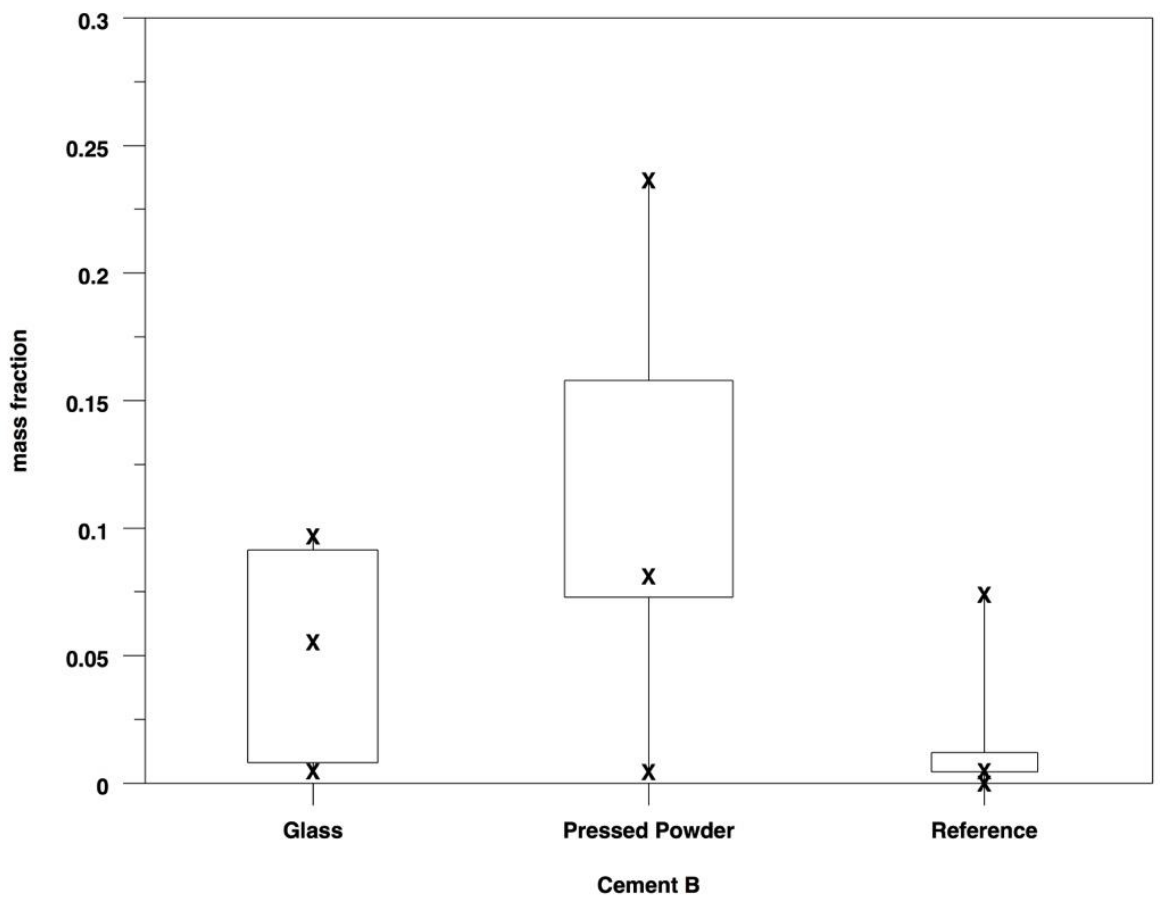

Figure 27 Box plots for CI XRF glass and powder, and reference methods. 
XRF Cl, Cements A B, Glass, Replicate 1, Material: 1*

\begin{tabular}{crrrrr}
\hline $\begin{array}{c}\text { Laboratory } \\
\text { Number }\end{array}$ & $\begin{array}{r}\text { Cell } \\
\text { Mean }\end{array}$ & $\begin{array}{c}\text { Cell } \\
\text { SD }\end{array}$ & $\boldsymbol{d}$ & $\boldsymbol{h}$ & $\boldsymbol{k}$ \\
\hline 1 & 0.0180 & 0.0030 & 0.0088 & 1.24 & 1.83 \\
2 & 0.0035 & 0.0010 & -0.0057 & -0.81 & 0.65 \\
3 & 0.0119 & 0.0007 & 0.0027 & 0.38 & 0.45 \\
4 & 0.0035 & 0.0002 & -0.0057 & -0.81 & 0.15 \\
\hline
\end{tabular}

Average of cell averages $\quad=\quad 0.00923$

Standard Deviation of cell averages $=0.00705$

Repeatability Standard Deviation $=0.00162$

Reproducibility Standard Deviation $=0.00721$

$\mathrm{h}, \mathrm{k}$ Critical Values $\quad=1.49000,1.60000$

XRF Cl, Cements A B, Glass, Replicate 1, Material: 2*

\begin{tabular}{crrrrr}
\hline $\begin{array}{c}\text { Laboratory } \\
\text { Number }\end{array}$ & $\begin{array}{r}\text { Cell } \\
\text { Mean }\end{array}$ & $\begin{array}{r}\text { Cell } \\
\text { SD }\end{array}$ & $\boldsymbol{d}$ & $\boldsymbol{h}$ & $\boldsymbol{k}$ \\
\hline 1 & 0.1222 & 0.0030 & 0.0523 & 1.07 & 0.77 \\
2 & 0.0578 & 0.0047 & -0.0120 & -0.24 & 1.21 \\
3 & 0.0917 & 0.0053 & 0.0219 & 0.45 & 1.36 \\
4 & 0.0076 & 0.0011 & -0.0622 & -1.27 & 0.29 \\
\hline
\end{tabular}

Average of cell averages $\quad=0.06983$

Standard Deviation of cell averages $=0.04910$

Repeatability Standard Deviation $=0.00388$

Reproducibility Standard Deviation $=0.04922$

$\mathrm{h}, \mathrm{k}$ Critical Values $\quad=1.49000,1.60000$

$* \mathrm{n}=4$ is less than recommended number of laboratories. These data are presented individually (glass and powder preparations) and collectively. 
XRF Cl, Cements A B, Glass, Replicate 2, Material: 1

\begin{tabular}{crrrrr}
\hline $\begin{array}{c}\text { Laboratory } \\
\text { Number }\end{array}$ & $\begin{array}{r}\text { Cell } \\
\text { Mean }\end{array}$ & $\begin{array}{r}\text { Cell } \\
\text { SD }\end{array}$ & $\boldsymbol{d}$ & $\boldsymbol{h}$ & $\boldsymbol{k}$ \\
\hline 1 & 0.0162 & 0.0032 & 0.0073 & 1.19 & 1.81 \\
2 & 0.0042 & 0.0012 & -0.0047 & -0.77 & 0.66 \\
3 & 0.0118 & 0.0009 & 0.0029 & 0.47 & 0.54 \\
4 & 0.0035 & 0.0002 & -0.0054 & -0.88 & 0.10 \\
\hline
\end{tabular}

Average of cell averages $\quad=\quad 0.00891$

Standard Deviation of cell averages $=0.00612$

Repeatability Standard Deviation $=0.00177$

Reproducibility Standard Deviation $=0.00633$

$\mathrm{h}, \mathrm{k}$ Critical Values $\quad=1.49000,1.60000$

XRF Cl, Cements A B, Glass, Replicate 2, Material: 2

\begin{tabular}{crrrrr}
\hline $\begin{array}{c}\text { Laboratory } \\
\text { Number }\end{array}$ & $\begin{array}{r}\text { Cell } \\
\text { Mean }\end{array}$ & $\begin{array}{r}\text { Cell } \\
\text { SD }\end{array}$ & $\boldsymbol{d}$ & $\boldsymbol{h}$ & $\boldsymbol{k}$ \\
\hline 1 & 0.1215 & 0.0015 & 0.0516 & 1.06 & 0.39 \\
2 & 0.0582 & 0.0052 & -0.0118 & -0.24 & 1.34 \\
3 & 0.0920 & 0.0055 & 0.0221 & 0.45 & 1.41 \\
4 & 0.0080 & 0.0009 & -0.0619 & -1.27 & 0.24 \\
\hline
\end{tabular}

Average of cell averages $\quad=0.06993$

Standard Deviation of cell averages $=0.04871$

Repeatability Standard Deviation $=0.00387$

Reproducibility Standard Deviation $=0.04883$

$\mathrm{h}, \mathrm{k}$ Critical Values $\quad=1.49000,1.60000$ 
XRF Cl, Cements A B, Powder, Replicate 1, Material: 1

\begin{tabular}{crrrrr}
\hline $\begin{array}{c}\text { Laboratory } \\
\text { Number }\end{array}$ & $\begin{array}{r}\text { Cell } \\
\text { Mean }\end{array}$ & $\begin{array}{r}\text { Cell } \\
\text { SD }\end{array}$ & $\boldsymbol{d}$ & $\boldsymbol{h}$ & $\boldsymbol{k}$ \\
\hline 1 & 0.0173 & 0.0008 & 0.0066 & 1.37 & 0.77 \\
2 & 0.0083 & 0.0007 & -0.0024 & -0.50 & 0.66 \\
3 & 0.0100 & 0.0000 & -0.0007 & -0.15 & 0.00 \\
4 & 0.0152 & 0.0023 & 0.0045 & 0.92 & 2.20 \\
5 & 0.0040 & 0.0000 & -0.0067 & -1.39 & 0.00 \\
6 & 0.0095 & 0.0004 & -0.0012 & -0.25 & 0.37 \\
\hline
\end{tabular}

Average of cell averages $\quad=\quad 0.01072$

Standard Deviation of cell averages $=0.00483$

Repeatability Standard Deviation $=0.00106$

Reproducibility Standard Deviation $=0.00493$

$\mathrm{h}, \mathrm{k}$ Critical Values $\quad=\quad 1.92000,1.68000$

XRF Cl, Cements A B, Powder, Replicate 1, Material: 2

\begin{tabular}{crrrrr}
\hline $\begin{array}{c}\text { Laboratory } \\
\text { Number }\end{array}$ & $\begin{array}{r}\text { Cell } \\
\text { Mean }\end{array}$ & $\begin{array}{r}\text { Cell } \\
\text { SD }\end{array}$ & $\boldsymbol{d}$ & $\boldsymbol{h}$ & $\boldsymbol{k}$ \\
\hline 1 & 0.1568 & 0.0017 & 0.0452 & 0.62 & 0.16 \\
2 & 0.0825 & 0.0019 & -0.0291 & -0.40 & 0.17 \\
3 & 0.1400 & 0.0000 & 0.0283 & 0.39 & 0.00 \\
4 & 0.2117 & 0.0266 & 0.1000 & 1.38 & 2.44 \\
5 & 0.0055 & 0.0000 & -0.1062 & -1.46 & 0.00 \\
6 & 0.0734 & 0.0007 & -0.0383 & -0.53 & 0.06 \\
\hline
\end{tabular}

Average of cell averages $\quad=\quad 0.11166$

Standard Deviation of cell averages $=0.07270$

Repeatability Standard Deviation $=0.01090$

Reproducibility Standard Deviation $=0.07338$

$\mathrm{h}, \mathrm{k}$ Critical Values $\quad=1.92000,1.68000$ 
XRF Cl, Cements A B, Powder, Replicate 2, Material: 1

\begin{tabular}{crrrrr}
\hline $\begin{array}{c}\text { Laboratory } \\
\text { Number }\end{array}$ & $\begin{array}{r}\text { Cell } \\
\text { Mean }\end{array}$ & $\begin{array}{r}\text { Cell } \\
\text { SD }\end{array}$ & $\boldsymbol{d}$ & $\boldsymbol{h}$ & $\boldsymbol{k}$ \\
\hline 1 & 0.0178 & 0.0012 & 0.0068 & 1.34 & 0.81 \\
2 & 0.0088 & 0.0008 & -0.0023 & -0.46 & 0.53 \\
3 & 0.0100 & 0.0000 & -0.0011 & -0.21 & 0.00 \\
4 & 0.0159 & 0.0032 & 0.0048 & 0.96 & 2.22 \\
5 & 0.0040 & 0.0000 & -0.0071 & -1.40 & 0.00 \\
6 & 0.0099 & 0.0005 & -0.0011 & -0.23 & 0.35 \\
\hline
\end{tabular}

Average of cell averages $\quad=\quad 0.01107$

Standard Deviation of cell averages $=0.00504$

Repeatability Standard Deviation $=0.00145$

Reproducibility Standard Deviation $=0.00521$

$\mathrm{h}, \mathrm{k}$ Critical Values $\quad=1.92000,1.68000$

XRF Cl, Cements A B, Powder, Replicate 2, Material: 2

\begin{tabular}{crrrrr}
\hline $\begin{array}{c}\text { Laboratory } \\
\text { Number }\end{array}$ & $\begin{array}{r}\text { Cell } \\
\text { Mean }\end{array}$ & $\begin{array}{r}\text { Cell } \\
\text { SD }\end{array}$ & $\boldsymbol{d}$ & $\boldsymbol{h}$ & $\boldsymbol{k}$ \\
\hline 1 & 0.1578 & 0.0016 & 0.0457 & 0.63 & 0.14 \\
2 & 0.0837 & 0.0019 & -0.0284 & -0.39 & 0.17 \\
3 & 0.1400 & 0.0000 & 0.0278 & 0.38 & 0.00 \\
4 & 0.2117 & 0.0269 & 0.0996 & 1.37 & 2.44 \\
5 & 0.0055 & 0.0000 & -0.1067 & -1.47 & 0.00 \\
6 & 0.0742 & 0.0008 & -0.0380 & -0.52 & 0.07 \\
\hline
\end{tabular}

Average of cell averages $\quad=\quad 0.11216$

Standard Deviation of cell averages $=0.07267$

Repeatability Standard Deviation $=0.01105$

Reproducibility Standard Deviation $=0.07337$

$\mathrm{h}, \mathrm{k}$ Critical Values $\quad=\quad 1.92000,1.68000$ 
XRF Cl, Cements A B, Combined Methods, Replicate 1, Material: 1

\begin{tabular}{crrrrr}
\hline $\begin{array}{c}\text { Laboratory } \\
\text { Number }\end{array}$ & $\begin{array}{r}\text { Cell } \\
\text { Mean }\end{array}$ & $\begin{array}{r}\text { Cell } \\
\text { SD }\end{array}$ & $\boldsymbol{d}$ & $\boldsymbol{h}$ & $\boldsymbol{k}$ \\
\hline 1 & 0.0035 & 0.0010 & -0.0057 & -1.05 & 1.02 \\
2 & 0.0119 & 0.0007 & 0.0027 & 0.51 & 0.71 \\
3 & 0.0035 & 0.0002 & -0.0056 & -1.05 & 0.24 \\
4 & 0.0173 & 0.0008 & 0.0082 & 1.52 & 0.79 \\
6 & 0.0083 & 0.0007 & -0.0009 & -0.16 & 0.68 \\
7 & 0.0152 & 0.0023 & 0.0060 & 1.12 & 2.27 \\
8 & 0.0040 & 0.0000 & -0.0052 & -0.96 & 0.00 \\
9 & 0.0095 & 0.0004 & 0.0004 & 0.07 & 0.38 \\
\hline
\end{tabular}

Average of cell averages $\quad=\quad 0.00916$

Standard Deviation of cell averages $=0.00537$

Repeatability Standard Deviation $=0.00103$

Reproducibility Standard Deviation $=0.00545$

$\mathrm{h}, \mathrm{k}$ Critical Values $\quad=\quad 2.15000,1.72000$

XRF Cl, Cements A B, Combined Methods, Replicate 1, Material: 2

\begin{tabular}{llllrl}
\hline Laboratory & $\begin{array}{l}\text { Cell } \\
\text { Number }\end{array}$ & Mean & Cell & \multicolumn{1}{l}{ SD } & \multicolumn{1}{l}{$l$} \\
\hline 1 & 0.0578 & 0.0047 & -0.0280 & -0.40 & 0.48 \\
2 & 0.0917 & 0.0053 & 0.0058 & 0.08 & 0.54 \\
3 & 0.0076 & 0.0011 & -0.0783 & -1.12 & 0.12 \\
4 & 0.1568 & 0.0017 & 0.0710 & 1.01 & 0.18 \\
6 & 0.0825 & 0.0019 & -0.0033 & -0.05 & 0.19 \\
7 & 0.2117 & 0.0266 & 0.1258 & 1.79 & 2.72 \\
8 & 0.0055 & 0.0000 & -0.0804 & -1.15 & 0.00 \\
9 & 0.0734 & 0.0007 & -0.0125 & -0.18 & 0.07 \\
\hline
\end{tabular}

Average of cell averages $\quad=\quad 0.08588$

Standard Deviation of cell averages $=0.07013$

Repeatability Standard Deviation $=0.00978$

Reproducibility Standard Deviation $=0.07070$

$\mathrm{h}, \mathrm{k}$ Critical Values $\quad=2.15000,1.72000$ 
Cl Glass, Replicate 1: $n=4$

\begin{tabular}{crrrrrr}
\hline Material & Xbar & $\boldsymbol{s}_{\boldsymbol{x}}$ & $\boldsymbol{s}_{\boldsymbol{r}}$ & $\boldsymbol{s}_{\boldsymbol{R}}$ & $\boldsymbol{r}$ & $\boldsymbol{R}$ \\
\hline 1 & 0.0092 & 0.0071 & 0.0016 & 0.0072 & 0.00 & 0.02 \\
2 & 0.0698 & 0.0491 & 0.0039 & 0.0492 & 0.01 & 0.14 \\
\hline
\end{tabular}

Cl Glass, Replicate 2: $n=4$

\begin{tabular}{crrrrrr}
\hline Material & Xbar & $\boldsymbol{s}_{\boldsymbol{x}}$ & $\boldsymbol{s}_{\boldsymbol{r}}$ & $\boldsymbol{s}_{\boldsymbol{R}}$ & $\boldsymbol{r}$ & $\boldsymbol{R}$ \\
\hline 1 & 0.0089 & 0.0061 & 0.0018 & 0.0063 & 0.00 & 0.02 \\
2 & 0.0699 & 0.0487 & 0.0039 & 0.0488 & 0.01 & 0.14 \\
\hline
\end{tabular}

Cl Powder, Replicate 1: $n=6$

\begin{tabular}{crrrrrr}
\hline Material & Xbar & $\boldsymbol{s}_{\boldsymbol{x}}$ & $\boldsymbol{s}_{\boldsymbol{r}}$ & $\boldsymbol{s}_{\boldsymbol{R}}$ & $\boldsymbol{r}$ & $\boldsymbol{R}$ \\
\hline 1 & 0.0107 & 0.0048 & 0.0011 & 0.0049 & 0.00 & 0.01 \\
2 & 0.1117 & 0.0727 & 0.0109 & 0.0734 & 0.03 & 0.21 \\
\hline
\end{tabular}

Cl Powder, Replicate 2: $n=6$

\begin{tabular}{crrrrrr}
\hline Material & $\mathbf{X b a r}$ & $\boldsymbol{s}_{\boldsymbol{x}}$ & $\boldsymbol{s}_{\boldsymbol{r}}$ & $\boldsymbol{s}_{\boldsymbol{R}}$ & $\boldsymbol{r}$ & $\boldsymbol{R}$ \\
\hline 1 & 0.0111 & 0.0050 & 0.0014 & 0.0052 & 0.00 & 0.01 \\
2 & 0.1122 & 0.0727 & 0.0110 & 0.0734 & 0.03 & 0.21 \\
\hline
\end{tabular}

Cl Combined Methods, Replicate 1: $n=9$

\begin{tabular}{crrrrrr}
\hline Material & Xbar & $\boldsymbol{s}_{\boldsymbol{x}}$ & $\boldsymbol{s}_{\boldsymbol{r}}$ & $\boldsymbol{s}_{\boldsymbol{R}}$ & $\boldsymbol{r}$ & $\boldsymbol{R}$ \\
\hline 1 & 0.0092 & 0.0054 & 0.0010 & 0.0054 & 0.00 & 0.02 \\
2 & 0.0859 & 0.0701 & 0.0098 & 0.0707 & 0.03 & 0.20 \\
\hline
\end{tabular}

Cl Combined Methods, Replicate 2: n=9

\begin{tabular}{crrrrrr}
\hline Material & $\mathbf{X b a r}$ & $\boldsymbol{s}_{\boldsymbol{x}}$ & $\boldsymbol{s}_{\boldsymbol{r}}$ & $\boldsymbol{s}_{\boldsymbol{R}}$ & $\boldsymbol{r}$ & $\boldsymbol{R}$ \\
\hline 1 & 0.0102 & 0.0053 & 0.0016 & 0.0055 & 0.00 & 0.02 \\
2 & 0.0953 & 0.0648 & 0.0089 & 0.0653 & 0.02 & 0.18 \\
\hline
\end{tabular}




\title{
Appendix B. Request for Participants Letter.
}

\author{
ASTM Round Robin for C01.23.03 Task Force Group
}

\section{Instructions}

The ASTM C01.23.03 task force group wishes to thank you for agreeing to be a part of the Round Robin (RR) which will define the precision and, hopefully, the bias of the X-ray fluorescence spectrometric (XRF) elemental analysis of hydraulic cements. Many versions of an XRF analytical procedure exist as set up and used by cement producers, cement users, commercial laboratories and general users. Generally, all parties are interested in producing a high quality, accurate analysis for industrial use. The Cement and Concrete Laboratory (CCRL) in cooperation with the ASTM Inter-Laboratory Study (ILS) has agreed to provide samples of Portland cement (meeting ASTM C150 guidelines) and various other mixtures of Portland cement and other compounds for the XRF analysis in this RR. Statistical analysis of the returned data will be compiled for the task force by NIST statisticians.

\section{Method}

The " $10^{\text {th }}$ Preliminary Draft" of "Standard Test Method for the Major and Minor Elements in Cement by X-Ray Fluorescence" produced by the task force group is available by down loading from a special web-based program set up by CCRL for the specific purpose of this RR. The method is specifically, a generalized approach to the XRF analysis of hydraulic cements, and is subject to revision when data is collected to show any confusion, indecision, or conflicts in the " $10^{\text {th }}$ Preliminary Draft." There have already been "lots" of "discussions" and changes to the method, and there may be more changes. Some have already been suggested by task force members and other interested parties that may be implemented when actual data begins to roll in.

In the interest of collecting data from the RR before I go to a nursing home, we should start to collect now. Again the method is a generalized approach to XRF analysis of hydraulic cements, and the individual Standard Operating Procedures (SOP) used in your individual laboratory should fit in the generalized guidelines. Please follow the method as closely as possible so that your specific laboratory data can be utilized for comparison to all the other data.

The method actually has two preparation schemes for presentation to the XRF, fused disk and pressed powder. If your laboratory has the capability to do both preparation methods, please, please, do so.

In Part 4.2.2 of the " $10^{\text {th }}$ Preliminary Draft," it specifically states "Part 2 of the method is not generally applicable to the analysis of blended cements, including those blended with silica fume, limestone, pozzolans, fly ash, slag, and any combination thereof higher than the amounts of additions found in Portland cements meeting Specification C 150." For the purposes of this RR data collection only, ignore this statement and run pressed 
pellets, if you have the capability, for comparison with the fused disk preparation. We need to establish the precision and truth of this statement and a comparison will determine if this statement is correct and help establish precision data on the pressed pellet mixtures by XRF.

\section{Data Return}

The data return for this RR is by accessing the CCRL web site for examination and determining what needs to be reported. It will be much easier if you report data on-line in a similar fashion as CCRL data is now reported; the statistician will love you. If you are unable to report data on-line, fill out the data and send to me (by FAX) and I'll key punch it in for your data return. By making a request to CCRL, on this RR you can obtain more sample, if you are going to do samples by pressed powder Ask for three vials of about 30 grams on each of the RR samples for powder prep and one sample vial if sample prep is fused disk only.

If you have the capability, we ask you to run powder prep and fused pellet on the two samples being mailed from CCRL. So for instance, there will be Sample A fused pellet data return and Sample A pressed pellet data return; then Sample B fused pellet data return and Sample B pressed pellet data return. There will be potentially 4 data return sheets for each sample in the mailing from CCRL. The first two samples mailed from CCRL are guaranteed to be $\mathrm{C} 150$ Portland Cements. The next two to four mailings of sample pairs will be mixtures.

If you can't do fused pellets, please participate in the pressed pellet RR, and conversely if you can't do pressed pellets, do fused pellets. All data will be used and gratefully appreciated by the task force group and task group chairman. We estimate that this RR study will take a minimum of $1 \frac{1 / 2}{2}$ years to $2^{1 / 2}$ years, a mere moment in the large ASTM scheme of things, but the data will hopefully live forever.

1. The section at the top should be filled out for company and individual returning the data, but, in general, no data will be identified with the lab unless you wish it to be. This ID is in case we have to contact you to clear up some misunderstanding or finger trouble with the data entry.

2. Please give as much data on the Fusion Bead preparation as possible. Flux composition, Non-wetting agent, etc. For the Pressed Pellet, as much info as possible, Binder composition, Grinding aid and etc.

3. Look at the return data sheet, what is intended here is to make on Day 1, three pellets: fusion and/or pressed pellets, and analyze each pellet twice, then report on the properly identified data sheets. Day 2 of your choosing, make three pellets: fusion and/or pressed pellets, and run each one twice, and report results on the proper data return sheets.

4. If possible (and only if possible) on your instrument, give the raw element intensities and/or raw background intensities before calculations and applied corrections. In the case 
of each element you only need to answer the Calibration/correction model question once, not every time on each prep.

5. LOI should be run and reported each sample prep day. Insoluble Residue and Free Lime need only to be run once, each prep day and only if the sufficient data is returned for the XRF data. The RR is primarily an XRF RR; the other data at the end of the CCRL data return will be used if needed.

6. Data should be reported to the CCRL web site with one more significant digit than the smallest uncertainty (or standard deviation) value of your best standard elemental value for the element being reported back to CCRL. Report determined values no later than three (3) months after receipt of samples in your laboratory. (i. e., SRM 1880a has a certified value for $\mathrm{CaO}$ of $63.83 \%+/-0.46$, report to three decimals for $\mathrm{CaO}$ unless you have lower values on reference materials that were used. $\mathrm{ZnO}$ is certified at $0.005 \%+/-0.001$, so report to four decimals in weight percent, unless you have used reference standards which have a lower + /- values.)

\section{LeRoy Jacobs}

ASTM C01.23.03 Task Force Group Chairman

Wyoming Analytical Lab, Inc.

1511 Washington Ave.

Golden, CO 80401

$\mathrm{Ph}$ (303) 278-2446

FAX (303) 278-2439

e:mail walxray@aol.com 
Appendix C. XRF Glass Summary by Material and Replicate.

\begin{tabular}{|c|c|c|c|c|c|c|c|c|}
\hline Analyte & Material & Xbar & $s_{x}$ & $s_{r}$ & $s_{R}$ & $r$ & $R$ & $\mathrm{n}$ \\
\hline \multirow[t]{4}{*}{$\mathrm{CaO}$} & 1 & 63.8787 & 0.3498 & 0.1061 & 0.363 & 0.3 & 1.02 & 17 \\
\hline & 2 & 63.5162 & 0.3384 & 0.1261 & 0.3574 & 0.35 & 1 & \\
\hline & 1 & 63.872 & 0.3628 & 0.1056 & 0.3754 & 0.3 & 1.05 & \\
\hline & 2 & 63.5022 & 0.3719 & 0.1344 & 0.3916 & 0.38 & 1.1 & \\
\hline \multirow{4}{*}{$\mathrm{SiO}_{2}$} & 1 & 20.535 & 0.075 & 0.0493 & 0.0875 & 0.14 & 0.25 & 18 \\
\hline & 2 & 20.2389 & 0.11 & 0.0574 & 0.1218 & 0.16 & 0.34 & \\
\hline & 1 & 20.5316 & 0.0818 & 0.0467 & 0.0922 & 0.13 & 0.26 & \\
\hline & 2 & 20.2336 & 0.1028 & 0.0607 & 0.1168 & 0.17 & 0.33 & \\
\hline \multirow[t]{4}{*}{$\mathrm{Al}_{2} \mathrm{O}_{3}$} & 1 & 5.0078 & 0.0495 & 0.0216 & 0.0532 & 0.06 & 0.15 & 17 \\
\hline & 2 & 5.13 & 0.0569 & 0.0223 & 0.0604 & 0.06 & 0.17 & \\
\hline & 1 & 5.0085 & 0.0498 & 0.0188 & 0.0526 & 0.05 & 0.15 & \\
\hline & 2 & 5.133 & 0.0581 & 0.0207 & 0.0611 & 0.06 & 0.17 & \\
\hline \multirow[t]{4}{*}{$\mathrm{Fe}_{2} \mathrm{O}_{3}$} & 1 & 2.7363 & 0.0283 & 0.0138 & 0.031 & 0.04 & 0.09 & 15 \\
\hline & 2 & 4.2401 & 0.0291 & 0.0108 & 0.0308 & 0.03 & 0.09 & \\
\hline & 1 & 2.7371 & 0.0287 & 0.0109 & 0.0303 & 0.03 & 0.08 & \\
\hline & 2 & 4.2399 & 0.0309 & 0.0173 & 0.0347 & 0.05 & 0.1 & \\
\hline \multirow[t]{4}{*}{$\mathrm{SO}_{3}$} & 1 & 2.8513 & 0.048 & 0.024 & 0.0527 & 0.07 & 0.15 & 16 \\
\hline & 2 & 3.5726 & 0.0769 & 0.025 & 0.0802 & 0.07 & 0.22 & \\
\hline & 1 & 2.8515 & 0.0482 & 0.0228 & 0.0525 & 0.06 & 0.15 & \\
\hline & 2 & 3.5639 & 0.0873 & 0.1044 & 0.1292 & 0.29 & 0.36 & \\
\hline \multirow[t]{4}{*}{$\mathrm{MgO}$} & 1 & 1.9053 & 0.0278 & 0.0146 & 0.0308 & 0.04 & 0.09 & 17 \\
\hline & 2 & 1.0653 & 0.0217 & 0.0096 & 0.0234 & 0.03 & 0.07 & \\
\hline & 1 & 1.9071 & 0.0272 & 0.0152 & 0.0306 & 0.04 & 0.09 & \\
\hline & 2 & 1.0648 & 0.0161 & 0.0087 & 0.018 & 0.02 & 0.05 & \\
\hline \multirow[t]{4}{*}{$\mathrm{Na}_{2} \mathrm{O}$} & 1 & 0.2098 & 0.014 & 0.0089 & 0.0162 & 0.02 & 0.05 & 15 \\
\hline & 2 & 0.0569 & 0.0227 & 0.0077 & 0.0238 & 0.02 & 0.07 & \\
\hline & 1 & 0.2098 & 0.0125 & 0.0083 & 0.0146 & 0.02 & 0.04 & \\
\hline & 2 & 0.0629 & 0.028 & 0.043 & 0.0482 & 0.12 & 0.13 & \\
\hline \multirow[t]{4}{*}{$\mathrm{K}_{2} \mathrm{O}$} & 1 & 0.6864 & 0.0097 & 0.0043 & 0.0104 & 0.01 & 0.03 & 17 \\
\hline & 2 & 0.3834 & 0.0051 & 0.0035 & 0.006 & 0.01 & 0.02 & \\
\hline & 1 & 0.6864 & 0.0101 & 0.0048 & 0.011 & 0.01 & 0.03 & \\
\hline & 2 & 0.383 & 0.0052 & 0.0034 & 0.0061 & 0.01 & 0.02 & \\
\hline \multirow[t]{4}{*}{$\mathrm{TiO}_{2}$} & 1 & 0.3095 & 0.0067 & 0.004 & 0.0076 & 0.01 & 0.02 & 17 \\
\hline & 2 & 0.334 & 0.0066 & 0.0044 & 0.0077 & 0.01 & 0.02 & \\
\hline & 1 & 0.3094 & 0.0067 & 0.0043 & 0.0078 & 0.01 & 0.02 & \\
\hline & 2 & 0.3333 & 0.0071 & 0.0044 & 0.0081 & 0.01 & 0.02 & \\
\hline
\end{tabular}




\begin{tabular}{ccrrrrrrr}
$\begin{array}{c}\text { continued } \\
\text { Analyte }\end{array}$ & Material & Xbar & $s_{x}$ & $s_{r}$ & $s_{R}$ & $r$ & $R$ & $\mathrm{n}$ \\
\hline $\mathrm{P}_{2} \mathrm{O}_{5}$ & 1 & 0.2126 & 0.0041 & 0.0041 & 0.0056 & 0.01 & 0.02 & 16 \\
& 2 & 0.0912 & 0.0014 & 0.0025 & 0.0027 & 0.01 & 0.01 & \\
& 1 & 0.213 & 0.0046 & 0.0036 & 0.0057 & 0.01 & 0.02 & \\
& 2 & 0.0918 & 0.0016 & 0.0025 & 0.0028 & 0.01 & 0.01 & \\
\hline $\mathrm{Cl}$ & 1 & 0.0092 & 0.0054 & 0.001 & 0.0054 & 0 & 0.02 & 9 \\
(glass+powder) & 2 & 0.0859 & 0.0701 & 0.0098 & 0.0707 & 0.03 & 0.2 &
\end{tabular}


Appendix D. XRF Powder Summary by Material and Replicate.

\begin{tabular}{|c|c|c|c|c|c|c|c|c|}
\hline Analyte & Material & Xbar & $s_{x}$ & $S_{r}$ & $s_{R}$ & $r$ & $R$ & $\mathrm{n}$ \\
\hline \multirow{4}{*}{$\mathrm{CaO}$} & 1 & 64.0931 & 0.3603 & 0.1207 & 0.3767 & 0.34 & 1.05 & 23 \\
\hline & 2 & 63.6979 & 0.281 & 0.1025 & 0.2962 & 0.29 & 0.83 & \\
\hline & 1 & 64.118 & 0.3618 & 0.1133 & 0.3763 & 0.32 & 1.05 & \\
\hline & 2 & 63.7197 & 0.2747 & 0.0961 & 0.2884 & 0.27 & 0.81 & \\
\hline \multirow[t]{4}{*}{$\mathrm{SiO}_{2}$} & 1 & 20.67 & 0.1258 & 0.0561 & 0.1358 & 0.16 & 0.38 & 22 \\
\hline & 2 & 20.117 & 0.176 & 0.0538 & 0.1827 & 0.15 & 0.51 & \\
\hline & 1 & 20.6776 & 0.1255 & 0.0584 & 0.1363 & 0.16 & 0.38 & \\
\hline & 2 & 20.1218 & 0.1756 & 0.0542 & 0.1824 & 0.15 & 0.51 & \\
\hline \multirow[t]{4}{*}{$\mathrm{Al}_{2} \mathrm{O}_{3}$} & 1 & 4.8213 & 0.0773 & 0.0239 & 0.0803 & 0.07 & 0.22 & 22 \\
\hline & 2 & 5.1292 & 0.1103 & 0.0196 & 0.1118 & 0.05 & 0.31 & \\
\hline & 1 & 4.8267 & 0.0763 & 0.0244 & 0.0795 & 0.07 & 0.22 & \\
\hline & 2 & 5.1321 & 0.1066 & 0.0203 & 0.1082 & 0.06 & 0.3 & \\
\hline \multirow[t]{4}{*}{$\mathrm{Fe}_{2} \mathrm{O}_{3}$} & 1 & 2.7444 & 0.044 & 0.0127 & 0.0455 & 0.04 & 0.13 & 24 \\
\hline & 2 & 4.2488 & 0.0422 & 0.0187 & 0.0456 & 0.05 & 0.13 & \\
\hline & 1 & 2.7442 & 0.0448 & 0.014 & 0.0466 & 0.04 & 0.13 & \\
\hline & 2 & 4.2475 & 0.0433 & 0.0181 & 0.0464 & 0.05 & 0.13 & \\
\hline \multirow[t]{4}{*}{$\mathrm{SO}_{3}$} & 1 & 2.9065 & 0.0912 & 0.0222 & 0.0934 & 0.06 & 0.26 & 24 \\
\hline & 2 & 3.5508 & 0.1393 & 0.0266 & 0.1414 & 0.07 & 0.4 & \\
\hline & 1 & 2.9065 & 0.092 & 0.0218 & 0.0941 & 0.06 & 0.26 & \\
\hline & 2 & 3.5521 & 0.138 & 0.0243 & 0.1398 & 0.07 & 0.39 & \\
\hline \multirow[t]{4}{*}{$\mathrm{MgO}$} & 1 & 1.8542 & 0.0655 & 0.0119 & 0.0664 & 0.03 & 0.19 & 26 \\
\hline & 2 & 1.1016 & 0.0627 & 0.0108 & 0.0634 & 0.03 & 0.18 & \\
\hline & 1 & 1.8541 & 0.0663 & 0.0128 & 0.0673 & 0.04 & 0.19 & \\
\hline & 2 & 1.0999 & 0.0625 & 0.0106 & 0.0633 & 0.03 & 0.18 & \\
\hline \multirow[t]{4}{*}{$\mathrm{Na}_{2} \mathrm{O}$} & 1 & 0.1862 & 0.0194 & 0.005 & 0.0199 & 0.01 & 0.06 & 24 \\
\hline & 2 & 0.0526 & 0.0182 & 0.006 & 0.019 & 0.02 & 0.05 & \\
\hline & 1 & 0.1868 & 0.0194 & 0.0053 & 0.02 & 0.01 & 0.06 & \\
\hline & 2 & 0.0521 & 0.0181 & 0.0051 & 0.0187 & 0.01 & 0.05 & \\
\hline \multirow[t]{4}{*}{$\mathrm{K}_{2} \mathrm{O}$} & 1 & 0.6824 & 0.0123 & 0.0047 & 0.013 & 0.01 & 0.04 & 21 \\
\hline & 2 & 0.3801 & 0.0105 & 0.0038 & 0.011 & 0.01 & 0.03 & \\
\hline & 1 & 0.6823 & 0.0125 & 0.0047 & 0.0132 & 0.01 & 0.04 & \\
\hline & 2 & 0.3801 & 0.0103 & 0.0036 & 0.0108 & 0.01 & 0.03 & \\
\hline \multirow[t]{4}{*}{$\mathrm{TiO}_{2}$} & 1 & 0.3019 & 0.0071 & 0.0029 & 0.0075 & 0.01 & 0.02 & 18 \\
\hline & 2 & 0.3322 & 0.0067 & 0.0029 & 0.0072 & 0.01 & 0.02 & \\
\hline & 1 & 0.3025 & 0.0069 & 0.0033 & 0.0075 & 0.01 & 0.02 & \\
\hline & 2 & 0.3323 & 0.0071 & 0.0033 & 0.0077 & 0.01 & 0.02 & \\
\hline
\end{tabular}




\begin{tabular}{ccrrrrrrrr}
$\begin{array}{c}\text { continued } \\
\text { Analyte }\end{array}$ & Material & Xbar & $s_{x}$ & $s_{r}$ & $s_{R}$ & $r$ & $R$ & \\
\cline { 1 - 8 } & 1 & 0.2163 & 0.0057 & 0.0034 & 0.0064 & 0.01 & 0.02 & 17 \\
& 2 & 0.0947 & 0.0081 & 0.0122 & 0.0138 & 0.03 & 0.04 & \\
& 1 & 0.2162 & 0.0054 & 0.0031 & 0.0061 & 0.01 & 0.02 & \\
& 2 & 0.0933 & 0.0043 & 0.0028 & 0.005 & 0.01 & 0.01 & \\
& 1 & 0.0095 & 0.0055 & 0.0014 & 0.0056 & 0 & 0.02 & 9 \\
(glass+powder) & 2 & 0.0864 & 0.0702 & 0.0099 & 0.0708 & 0.03 & 0.2 &
\end{tabular}




\section{Appendix E. Raw Data by Analyte.}

Data by oxide after initial outlier removal for $\mathrm{SiO}_{2}, \mathrm{Al}_{2} \mathrm{O}_{3}, \mathrm{Fe}_{2} \mathrm{O}_{3}, \mathrm{CaO}, \mathrm{MgO}, \mathrm{SO}_{3}, \mathrm{Na}_{2} \mathrm{O}$, $\mathrm{K}_{2} \mathrm{O}, \mathrm{TiO}_{2}$, and $\mathrm{Cl}$. Data are organized with the XRF-glass results to the left and XRFpowder to the right. A lab identifier is provided in the first column of each preparation method. The six replicate measurements for that lab and analyte for duplicate 1 and then duplicate two are provided in columns 2 and 3 of each preparation method. Duplicate measurements represent a repeat measurement on the same specimen. The final column labeled cement indicates cement A (1) or cement B (2). Since outliers were evaluated on an analyte-by-analyte basis, a specific lab designation may not be the same lab across all analytes. For example, if lab 1 had a high between-lab precision for $\mathrm{Al}_{2} \mathrm{O}_{3}$, its data were removed and the labs reordered so no gap in the numbering existed.

\begin{tabular}{|c|c|c|c|c|c|c|c|c|}
\hline \multirow{2}{*}{\multicolumn{2}{|c|}{$\begin{array}{l}\mathrm{SiO}_{2} \\
\mathrm{SiO2} \text { : Glass } \\
\text { Preparation }\end{array}$}} & \multirow[b]{2}{*}{$\begin{array}{l}\text { Duplicate } \\
2\end{array}$} & \multirow[b]{2}{*}{$\begin{array}{l}\text { Cemen } \\
\mathrm{t}\end{array}$} & \multicolumn{5}{|c|}{$\begin{array}{l}\text { SiO2: Powder } \\
\text { Preparation }\end{array}$} \\
\hline & & & & Lab & Duplicate 1 & $\begin{array}{l}\text { Duplicate } \\
2\end{array}$ & $\begin{array}{l}\text { Cen } \\
t\end{array}$ & \\
\hline 1 & 20.418 & 20.414 & 1 & & 1 & 20.650 & 20.680 & 1 \\
\hline 1 & 20.380 & 20.403 & 1 & & 1 & 20.800 & 20.800 & 1 \\
\hline 1 & 20.287 & 20.270 & 1 & & 1 & 20.760 & 20.780 & 1 \\
\hline 1 & 20.330 & 20.460 & 1 & & 1 & 20.570 & 20.630 & 1 \\
\hline 1 & 20.484 & 20.347 & 1 & & 1 & 20.630 & 20.730 & 1 \\
\hline 1 & 20.480 & 20.393 & 1 & & 1 & 20.730 & 20.730 & 1 \\
\hline 2 & 20.522 & 20.462 & 1 & & 2 & 20.542 & 20.575 & 1 \\
\hline 2 & 20.362 & 20.308 & 1 & & 2 & 20.580 & 20.580 & 1 \\
\hline 2 & 20.348 & 20.328 & 1 & & 2 & 20.569 & 20.581 & 1 \\
\hline 2 & 20.317 & 20.246 & 1 & & 2 & 20.636 & 20.654 & 1 \\
\hline 2 & 20.321 & 20.394 & 1 & & 2 & 20.684 & 20.645 & 1 \\
\hline 2 & 20.431 & 20.408 & 1 & & 2 & 20.645 & 20.370 & 1 \\
\hline 3 & 20.519 & 20.524 & 1 & & 3 & 20.565 & 20.572 & 1 \\
\hline 3 & 20.518 & 20.524 & 1 & & 3 & 20.726 & 20.715 & 1 \\
\hline 3 & 20.524 & 20.545 & 1 & & 3 & 20.595 & 20.619 & 1 \\
\hline 3 & 20.565 & 20.561 & 1 & & 3 & 20.634 & 20.628 & 1 \\
\hline 3 & 20.536 & 20.536 & 1 & & 3 & 20.647 & 20.669 & 1 \\
\hline 3 & 20.518 & 20.552 & 1 & & 3 & 20.635 & 20.636 & 1 \\
\hline 4 & 20.540 & 20.510 & 1 & & 4 & 20.640 & 20.660 & 1 \\
\hline 4 & 20.510 & 20.570 & 1 & & 4 & 20.640 & 20.630 & 1 \\
\hline 4 & 20.510 & 20.510 & 1 & & 4 & 20.600 & 20.590 & 1 \\
\hline 4 & 20.550 & 20.520 & 1 & & 4 & 20.700 & 20.720 & 1 \\
\hline 4 & 20.530 & 20.510 & 1 & & 4 & 20.550 & 20.540 & 1 \\
\hline 4 & 20.520 & 20.520 & 1 & & 4 & 20.640 & 20.630 & 1 \\
\hline 5 & 20.596 & 20.591 & 1 & & 5 & 20.590 & 20.640 & 1 \\
\hline 5 & 20.541 & 20.560 & 1 & & 5 & 20.690 & 20.760 & 1 \\
\hline 5 & 20.595 & 20.580 & 1 & & 5 & 20.760 & 20.790 & 1 \\
\hline 5 & 20.655 & 20.673 & 1 & & 5 & 20.570 & 20.580 & 1 \\
\hline 5 & 20.582 & 20.585 & 1 & & 5 & 20.690 & 20.630 & 1 \\
\hline 5 & 20.634 & 20.633 & 1 & & 5 & 20.670 & 20.660 & 1 \\
\hline 6 & 20.530 & 20.570 & 1 & & 6 & 20.940 & 20.930 & 1 \\
\hline
\end{tabular}




\begin{tabular}{|c|c|c|c|c|c|c|}
\hline 6 & 20.560 & 20.560 & 1 & 6 & 20.950 & 20.940 \\
\hline 6 & 20.560 & 20.580 & 1 & 6 & 20.900 & 20.910 \\
\hline 6 & 20.550 & 20.520 & 1 & 6 & 20.950 & 20.940 \\
\hline 6 & 20.470 & 20.440 & 1 & 6 & 20.920 & 20.910 \\
\hline 6 & 20.500 & 20.440 & 1 & 6 & 20.940 & 20.930 \\
\hline 7 & 20.660 & 20.670 & 1 & 7 & 20.760 & 20.780 \\
\hline 7 & 20.740 & 20.720 & 1 & 7 & 20.800 & 20.820 \\
\hline 7 & 20.590 & 20.590 & 1 & 7 & 20.760 & 20.780 \\
\hline 7 & 20.590 & 20.580 & 1 & 7 & 20.830 & 20.840 \\
\hline 7 & 20.760 & 20.740 & 1 & 7 & 20.760 & 20.770 \\
\hline 7 & 20.640 & 20.640 & 1 & 7 & 20.720 & 20.750 \\
\hline 8 & 20.552 & 20.552 & 1 & 8 & 20.740 & 20.750 \\
\hline 8 & 20.508 & 20.554 & 1 & 8 & 20.740 & 20.760 \\
\hline 8 & 20.499 & 20.475 & 1 & 8 & 20.790 & 20.750 \\
\hline 8 & 20.469 & 20.441 & 1 & 8 & 20.760 & 20.760 \\
\hline 8 & 20.472 & 20.455 & 1 & 8 & 20.740 & 20.710 \\
\hline 8 & 20.512 & 20.501 & 1 & 8 & 20.730 & 20.740 \\
\hline 9 & 20.500 & 20.490 & 1 & 9 & 20.420 & 20.440 \\
\hline 9 & 20.550 & 20.450 & 1 & 9 & 20.420 & 20.440 \\
\hline 9 & 20.560 & 20.500 & 1 & 9 & 20.410 & 20.440 \\
\hline 9 & 20.490 & 20.480 & 1 & 9 & 20.520 & 20.540 \\
\hline 9 & 20.510 & 20.530 & 1 & 9 & 20.430 & 20.450 \\
\hline 9 & 20.490 & 20.450 & 1 & 9 & 20.430 & 20.460 \\
\hline 10 & 20.560 & 20.470 & 1 & 10 & 20.766 & 20.751 \\
\hline 10 & 20.470 & 20.490 & 1 & 10 & 20.751 & 20.750 \\
\hline 10 & 20.460 & 20.500 & 1 & 10 & 20.768 & 20.769 \\
\hline 10 & 20.560 & 20.550 & 1 & 10 & 20.750 & 20.747 \\
\hline 10 & 20.570 & 20.570 & 1 & 10 & 20.695 & 20.692 \\
\hline 10 & 20.610 & 20.500 & 1 & 10 & 20.777 & 20.766 \\
\hline 11 & 20.430 & 20.460 & 1 & 11 & 20.527 & 20.529 \\
\hline 11 & 20.420 & 20.450 & 1 & 11 & 20.509 & 20.511 \\
\hline 11 & 20.500 & 20.520 & 1 & 11 & 20.538 & 20.503 \\
\hline 11 & 20.530 & 20.520 & 1 & 11 & 20.556 & 20.600 \\
\hline 11 & 20.480 & 20.460 & 1 & 11 & 20.603 & 20.593 \\
\hline 11 & 20.430 & 20.470 & 1 & 11 & 20.575 & 20.552 \\
\hline 12 & 20.550 & 20.550 & 1 & 12 & 20.560 & 20.570 \\
\hline 12 & 20.570 & 20.570 & 1 & 12 & 20.620 & 20.640 \\
\hline 12 & 20.540 & 20.480 & 1 & 12 & 20.690 & 20.650 \\
\hline 12 & 20.610 & 20.620 & 1 & 12 & 20.800 & 20.800 \\
\hline 12 & 20.620 & 20.580 & 1 & 12 & 20.730 & 20.780 \\
\hline 12 & 20.600 & 20.540 & 1 & 12 & 20.690 & 20.730 \\
\hline 13 & 20.496 & 20.542 & 1 & 13 & 20.556 & 20.592 \\
\hline 13 & 20.507 & 20.583 & 1 & 13 & 20.574 & 20.619 \\
\hline 13 & 20.516 & 20.609 & 1 & 13 & 20.550 & 20.608 \\
\hline 13 & 20.617 & 20.608 & 1 & 13 & 20.614 & 20.658 \\
\hline 13 & 20.627 & 20.646 & 1 & 13 & 20.629 & 20.647 \\
\hline 13 & 20.633 & 20.682 & 1 & 13 & 20.564 & 20.604 \\
\hline 14 & 20.480 & 20.520 & 1 & 14 & 20.510 & 20.532 \\
\hline 14 & 20.510 & 20.520 & 1 & 14 & 20.690 & 20.706 \\
\hline
\end{tabular}




\begin{tabular}{|c|c|c|c|c|c|c|}
\hline 14 & 20.500 & 20.490 & 1 & 14 & 20.624 & 20.639 \\
\hline 14 & 20.570 & 20.450 & 1 & 14 & 20.678 & 20.709 \\
\hline 14 & 20.570 & 20.500 & 1 & 14 & 20.560 & 20.577 \\
\hline 14 & 20.510 & 20.540 & 1 & 14 & 20.601 & 20.605 \\
\hline 15 & 20.550 & 20.570 & 1 & 15 & 20.590 & 20.610 \\
\hline 15 & 20.510 & 20.520 & 1 & 15 & 20.680 & 20.690 \\
\hline 15 & 20.430 & 20.480 & 1 & 15 & 20.740 & 20.740 \\
\hline 15 & 20.530 & 20.540 & 1 & 15 & 20.580 & 20.550 \\
\hline 15 & 20.630 & 20.630 & 1 & 15 & 20.640 & 20.710 \\
\hline 15 & 20.620 & 20.630 & 1 & 15 & 20.650 & 20.600 \\
\hline 16 & 20.640 & 20.640 & 1 & 16 & 20.530 & 20.560 \\
\hline 16 & 20.710 & 20.670 & 1 & 16 & 20.510 & 20.470 \\
\hline 16 & 20.680 & 20.660 & 1 & 16 & 20.510 & 20.560 \\
\hline 16 & 20.630 & 20.700 & 1 & 16 & 20.450 & 20.530 \\
\hline 16 & 20.670 & 20.650 & 1 & 16 & 20.480 & 20.500 \\
\hline 16 & 20.650 & 20.600 & 1 & 16 & 20.470 & 20.500 \\
\hline 17 & 20.489 & 20.479 & 1 & 17 & 20.650 & 20.630 \\
\hline 17 & 20.537 & 20.532 & 1 & 17 & 20.500 & 20.490 \\
\hline 17 & 20.446 & 20.511 & 1 & 17 & 20.600 & 20.600 \\
\hline 17 & 20.490 & 20.528 & 1 & 17 & 20.570 & 20.560 \\
\hline 17 & 20.514 & 20.474 & 1 & 17 & 20.420 & 20.420 \\
\hline 17 & 20.495 & 20.494 & 1 & 17 & 20.560 & 20.510 \\
\hline 18 & 20.600 & 20.610 & 1 & 18 & 20.502 & 20.536 \\
\hline 18 & 20.600 & 20.660 & 1 & 18 & 20.578 & 20.603 \\
\hline 18 & 20.600 & 20.630 & 1 & 18 & 20.614 & 20.613 \\
\hline 18 & 20.570 & 20.580 & 1 & 18 & 20.463 & 20.463 \\
\hline 18 & 20.610 & 20.670 & 1 & 18 & 20.531 & 20.518 \\
\hline 18 & 20.650 & 20.600 & 1 & 18 & 20.571 & 20.610 \\
\hline 1 & 20.236 & 20.296 & 2 & 19 & 20.710 & 20.770 \\
\hline 1 & 20.254 & 20.121 & 2 & 19 & 20.780 & 20.820 \\
\hline 1 & 20.079 & 20.093 & 2 & 19 & 20.780 & 20.770 \\
\hline 1 & 20.267 & 20.168 & 2 & 19 & 20.870 & 20.870 \\
\hline 1 & 20.304 & 20.118 & 2 & 19 & 20.880 & 20.910 \\
\hline 1 & 20.279 & 20.108 & 2 & 19 & 20.890 & 20.940 \\
\hline 2 & 20.319 & 20.198 & 2 & 20 & 20.880 & 20.820 \\
\hline 2 & 20.325 & 20.325 & 2 & 20 & 20.870 & 20.850 \\
\hline 2 & 20.126 & 20.237 & 2 & 20 & 20.720 & 20.780 \\
\hline 2 & 20.240 & 20.151 & 2 & 20 & 20.790 & 20.810 \\
\hline 2 & 20.172 & 20.063 & 2 & 20 & 20.830 & 20.850 \\
\hline 2 & 20.144 & 20.029 & 2 & 20 & 20.680 & 20.720 \\
\hline 3 & 20.163 & 20.168 & 2 & 21 & 20.714 & 20.653 \\
\hline 3 & 20.168 & 20.184 & 2 & 21 & 20.777 & 20.782 \\
\hline 3 & 20.188 & 20.195 & 2 & 21 & 20.669 & 20.679 \\
\hline 3 & 20.139 & 20.137 & 2 & 21 & 20.665 & 20.660 \\
\hline 3 & 20.148 & 20.145 & 2 & 21 & 20.706 & 20.653 \\
\hline 3 & 20.161 & 20.146 & 2 & 21 & 20.702 & 20.683 \\
\hline 4 & 20.250 & 20.250 & 2 & 22 & 20.840 & 20.880 \\
\hline 4 & 20.290 & 20.300 & 2 & 22 & 20.860 & 20.870 \\
\hline 4 & 20.300 & 20.290 & 2 & 22 & 20.910 & 20.870 \\
\hline
\end{tabular}




\begin{tabular}{|c|c|c|c|c|c|c|}
\hline 4 & 20.300 & 20.340 & 2 & 22 & 20.890 & 20.930 \\
\hline 4 & 20.280 & 20.280 & 2 & 22 & 20.960 & 20.970 \\
\hline 4 & 20.350 & 20.310 & 2 & 22 & 20.920 & 20.940 \\
\hline 5 & 20.307 & 20.297 & 2 & 1 & 20.100 & 20.140 \\
\hline 5 & 20.315 & 20.273 & 2 & 1 & 20.150 & 20.180 \\
\hline 5 & 20.321 & 20.308 & 2 & 1 & 20.150 & 20.130 \\
\hline 5 & 20.356 & 20.352 & 2 & 1 & 20.120 & 20.150 \\
\hline 5 & 20.376 & 20.394 & 2 & 1 & 20.140 & 20.120 \\
\hline 5 & 20.357 & 20.387 & 2 & 1 & 20.200 & 20.160 \\
\hline 6 & 20.390 & 20.270 & 2 & 2 & 19.691 & 19.722 \\
\hline 6 & 20.360 & 20.290 & 2 & 2 & 19.678 & 19.722 \\
\hline 6 & 20.340 & 20.410 & 2 & 2 & 19.737 & 19.728 \\
\hline 6 & 20.220 & 20.200 & 2 & 2 & 19.740 & 19.742 \\
\hline 6 & 20.260 & 20.200 & 2 & 2 & 19.712 & 19.752 \\
\hline 6 & 20.220 & 20.240 & 2 & 2 & 19.808 & 19.820 \\
\hline 7 & 20.240 & 20.240 & 2 & 3 & 20.108 & 20.130 \\
\hline 7 & 20.350 & 20.360 & 2 & 3 & 20.113 & 20.128 \\
\hline 7 & 20.440 & 20.440 & 2 & 3 & 19.923 & 19.938 \\
\hline 7 & 20.310 & 20.360 & 2 & 3 & 20.128 & 20.109 \\
\hline 7 & 20.450 & 20.450 & 2 & 3 & 20.013 & 20.025 \\
\hline 7 & 20.320 & 20.320 & 2 & 3 & 20.118 & 20.144 \\
\hline 8 & 20.231 & 20.214 & 2 & 4 & 20.240 & 20.260 \\
\hline 8 & 20.272 & 20.243 & 2 & 4 & 20.280 & 20.250 \\
\hline 8 & 20.234 & 20.270 & 2 & 4 & 20.290 & 20.290 \\
\hline 8 & 20.227 & 20.219 & 2 & 4 & 20.230 & 20.200 \\
\hline 8 & 20.228 & 20.223 & 2 & 4 & 20.300 & 20.280 \\
\hline 8 & 20.276 & 20.257 & 2 & 4 & 20.250 & 20.240 \\
\hline 9 & 20.200 & 20.200 & 2 & 5 & 20.360 & 20.350 \\
\hline 9 & 20.200 & 20.190 & 2 & 5 & 20.270 & 20.270 \\
\hline 9 & 20.160 & 20.170 & 2 & 5 & 20.240 & 20.310 \\
\hline 9 & 20.210 & 20.270 & 2 & 5 & 20.300 & 20.310 \\
\hline 9 & 20.190 & 20.300 & 2 & 5 & 20.320 & 20.290 \\
\hline 9 & 20.170 & 20.200 & 2 & 5 & 20.350 & 20.280 \\
\hline 10 & 20.230 & 20.140 & 2 & 6 & 20.400 & 20.410 \\
\hline 10 & 20.180 & 20.250 & 2 & 6 & 20.350 & 20.330 \\
\hline 10 & 20.160 & 20.160 & 2 & 6 & 20.450 & 20.440 \\
\hline 10 & 20.240 & 20.220 & 2 & 6 & 20.300 & 20.310 \\
\hline 10 & 20.240 & 20.300 & 2 & 6 & 20.340 & 20.320 \\
\hline 10 & 20.120 & 20.120 & 2 & 6 & 20.410 & 20.420 \\
\hline 11 & 19.970 & 19.970 & 2 & 7 & 20.480 & 20.500 \\
\hline 11 & 19.970 & 19.960 & 2 & 7 & 20.530 & 20.560 \\
\hline 11 & 20.050 & 19.850 & 2 & 7 & 20.480 & 20.510 \\
\hline 11 & 19.890 & 20.090 & 2 & 7 & 20.510 & 20.520 \\
\hline 11 & 19.880 & 20.020 & 2 & 7 & 20.590 & 20.590 \\
\hline 11 & 19.960 & 19.990 & 2 & 7 & 20.540 & 20.550 \\
\hline 12 & 20.250 & 20.200 & 2 & 8 & 20.060 & 20.040 \\
\hline 12 & 20.260 & 20.210 & 2 & 8 & 20.060 & 20.040 \\
\hline 12 & 20.260 & 20.200 & 2 & 8 & 20.000 & 19.970 \\
\hline 12 & 20.310 & 20.270 & 2 & 8 & 20.100 & 20.080 \\
\hline
\end{tabular}




\begin{tabular}{lll}
12 & 20.300 & 20.220 \\
12 & 20.310 & 20.240 \\
13 & 20.009 & 20.224 \\
13 & 20.073 & 20.160 \\
13 & 20.066 & 20.189 \\
13 & 20.173 & 20.182 \\
13 & 20.162 & 20.195 \\
13 & 20.193 & 20.115 \\
14 & 20.300 & 20.300 \\
14 & 20.290 & 20.300 \\
14 & 20.310 & 20.320 \\
14 & 20.210 & 20.290 \\
14 & 20.290 & 20.260 \\
14 & 20.260 & 20.250 \\
15 & 20.220 & 20.200 \\
15 & 20.180 & 20.170 \\
15 & 20.120 & 20.130 \\
15 & 20.310 & 20.280 \\
15 & 20.430 & 20.430 \\
15 & 20.340 & 20.340 \\
16 & 20.460 & 20.430 \\
16 & 20.460 & 20.430 \\
16 & 20.540 & 20.490 \\
16 & 20.420 & 20.400 \\
16 & 20.430 & 20.450 \\
16 & 20.400 & 20.440 \\
17 & 20.134 & 20.143 \\
17 & 20.134 & 20.143 \\
17 & 20.105 & 20.123 \\
17 & 20.102 & 20.135 \\
17 & 20.102 & 20.135 \\
17 & 20.146 & 20.101 \\
18 & 20.280 & 20.300 \\
18 & 20.300 & 20.250 \\
18 & 20.290 & 20.310 \\
18 & 20.310 & 20.350 \\
18 & 20.320 & 20.340 \\
18 & 20.340 & 20.310 \\
\hline & & \\
18 & &
\end{tabular}

\begin{tabular}{|c|c|c|c|}
\hline 2 & 8 & 20.100 & 20.080 \\
\hline 2 & 8 & 19.950 & 19.970 \\
\hline 2 & 9 & 19.940 & 19.970 \\
\hline 2 & 9 & 19.900 & 19.910 \\
\hline 2 & 9 & 19.830 & 19.850 \\
\hline 2 & 9 & 19.940 & 19.950 \\
\hline 2 & 9 & 19.890 & 19.890 \\
\hline 2 & 9 & 19.880 & 19.900 \\
\hline 2 & 10 & 20.394 & 20.403 \\
\hline 2 & 10 & 20.438 & 20.435 \\
\hline 2 & 10 & 20.423 & 20.432 \\
\hline 2 & 10 & 20.309 & 20.308 \\
\hline 2 & 10 & 20.418 & 20.430 \\
\hline 2 & 10 & 20.431 & 20.430 \\
\hline 2 & 11 & 20.013 & 20.013 \\
\hline 2 & 11 & 19.939 & 19.935 \\
\hline 2 & 11 & 19.995 & 19.982 \\
\hline 2 & 11 & 19.981 & 19.963 \\
\hline 2 & 11 & 19.995 & 19.997 \\
\hline 2 & 11 & 20.021 & 20.035 \\
\hline 2 & 12 & 19.980 & 20.060 \\
\hline 2 & 12 & 20.130 & 20.170 \\
\hline 2 & 12 & 20.200 & 20.220 \\
\hline 2 & 12 & 20.050 & 19.990 \\
\hline 2 & 12 & 20.040 & 20.060 \\
\hline 2 & 12 & 20.100 & 20.090 \\
\hline 2 & 13 & 20.113 & 20.154 \\
\hline 2 & 13 & 20.080 & 20.099 \\
\hline 2 & 13 & 20.082 & 20.108 \\
\hline 2 & 13 & 20.041 & 20.079 \\
\hline 2 & 13 & 20.064 & 20.102 \\
\hline 2 & 13 & 20.037 & 20.070 \\
\hline 2 & 14 & 19.936 & 19.968 \\
\hline 2 & 14 & 19.925 & 19.928 \\
\hline 2 & 14 & 19.927 & 19.946 \\
\hline 2 & 14 & 20.026 & 20.056 \\
\hline 2 & 14 & 19.947 & 19.966 \\
\hline 2 & 14 & 20.101 & 20.101 \\
\hline & 15 & 20.160 & 20.130 \\
\hline & 15 & 20.100 & 20.030 \\
\hline & 15 & 20.090 & 20.050 \\
\hline & 15 & 20.030 & 20.020 \\
\hline & 15 & 20.080 & 20.010 \\
\hline & 15 & 20.050 & 19.960 \\
\hline & 16 & 20.090 & 20.110 \\
\hline & 16 & 20.130 & 20.150 \\
\hline & 16 & 20.160 & 20.150 \\
\hline & 16 & 20.050 & 20.050 \\
\hline & 16 & 20.060 & 20.090 \\
\hline
\end{tabular}




\begin{tabular}{|llll}
16 & 20.060 & 20.040 & 2 \\
17 & 20.040 & 20.000 & 2 \\
17 & 20.040 & 20.010 & 2 \\
17 & 20.090 & 20.110 & 2 \\
17 & 19.900 & 19.900 & 2 \\
17 & 19.900 & 19.900 & 2 \\
17 & 19.910 & 19.910 & 2 \\
18 & 20.058 & 20.080 & 2 \\
18 & 20.056 & 20.057 & 2 \\
18 & 20.000 & 19.955 & 2 \\
18 & 20.037 & 20.054 & 2 \\
18 & 20.135 & 20.123 & 2 \\
18 & 20.176 & 20.179 & 2 \\
19 & 20.090 & 20.180 & 2 \\
19 & 20.140 & 20.180 & 2 \\
19 & 20.190 & 20.150 & 2 \\
19 & 20.150 & 20.220 & 2 \\
19 & 20.200 & 20.220 & 2 \\
19 & 20.200 & 20.260 & 2 \\
20 & 19.980 & 19.970 & 2 \\
20 & 20.040 & 20.030 & 2 \\
20 & 20.020 & 20.090 & 2 \\
20 & 20.080 & 20.060 & 2 \\
20 & 20.130 & 20.140 & 2 \\
20 & 20.030 & 20.020 & 2 \\
21 & 20.015 & 20.008 & 2 \\
21 & 20.015 & 20.008 & 2 \\
21 & 20.093 & 20.075 & 2 \\
21 & 20.121 & 20.152 & 2 \\
21 & 20.121 & 20.152 & 2 \\
21 & 20.157 & 20.130 & 2 \\
22 & 20.110 & 20.130 & 2 \\
22 & 20.180 & 20.200 & 2 \\
22 & 20.210 & 20.230 & 2 \\
22 & 20.160 & 20.190 & 2 \\
22 & 20.160 & 20.180 & 2 \\
22 & 20.230 & 20.230 & 2
\end{tabular}




\begin{tabular}{|c|c|c|c|c|c|c|c|}
\hline \multicolumn{4}{|c|}{ Al203: Glass Preparation } & \multicolumn{4}{|c|}{ Al203: Powder Preparation } \\
\hline Lab & Duplicate 1 & Duplicate 2 & Cement & Lab & Duplicate 1 & Duplicate 2 & Cement \\
\hline 1 & 2.7050 & 2.7210 & 1 & 1 & 2.6600 & 2.6600 & 1 \\
\hline 1 & 2.7270 & 2.7210 & 1 & 1 & 2.6600 & 2.6600 & 1 \\
\hline 1 & 2.7170 & 2.7210 & 1 & 1 & 2.6500 & 2.6400 & 1 \\
\hline 1 & 2.7340 & 2.7240 & 1 & 1 & 2.6600 & 2.6600 & 1 \\
\hline 1 & 2.7510 & 2.7270 & 1 & 1 & 2.6700 & 2.6500 & 1 \\
\hline 1 & 2.7340 & 2.7360 & 1 & 1 & 2.6600 & 2.6500 & 1 \\
\hline 2 & 2.6650 & 2.6670 & 1 & 2 & 2.6800 & 2.6800 & 1 \\
\hline 2 & 2.7350 & 2.7360 & 1 & 2 & 2.7100 & 2.7000 & 1 \\
\hline 2 & 2.7430 & 2.7400 & 1 & 2 & 2.7400 & 2.7400 & 1 \\
\hline 2 & 2.7350 & 2.7460 & 1 & 2 & 2.7000 & 2.6700 & 1 \\
\hline 2 & 2.7590 & 2.7550 & 1 & 2 & 2.7100 & 2.7200 & 1 \\
\hline 2 & 2.7510 & 2.7560 & 1 & 2 & 2.7100 & 2.6500 & 1 \\
\hline 3 & 2.7230 & 2.7260 & 1 & 4 & 2.7040 & 2.7060 & 1 \\
\hline 3 & 2.7260 & 2.7270 & 1 & 4 & 2.6910 & 2.6910 & 1 \\
\hline 3 & 2.7300 & 2.7280 & 1 & 4 & 2.7170 & 2.7190 & 1 \\
\hline 3 & 2.7310 & 2.7320 & 1 & 4 & 2.7390 & 2.7430 & 1 \\
\hline 3 & 2.7310 & 2.7320 & 1 & 4 & 2.7400 & 2.7410 & 1 \\
\hline 3 & 2.7310 & 2.7320 & 1 & 4 & 2.7400 & 2.7370 & 1 \\
\hline 4 & 2.7300 & 2.7300 & 1 & 5 & 2.7400 & 2.7400 & 1 \\
\hline 4 & 2.7300 & 2.7300 & 1 & 5 & 2.7400 & 2.7400 & 1 \\
\hline 4 & 2.7300 & 2.7300 & 1 & 5 & 2.7400 & 2.7400 & 1 \\
\hline 4 & 2.7300 & 2.7300 & 1 & 5 & 2.7400 & 2.7500 & 1 \\
\hline 4 & 2.7300 & 2.7300 & 1 & 5 & 2.7400 & 2.7400 & 1 \\
\hline 4 & 2.7400 & 2.7400 & 1 & 5 & 2.7300 & 2.7300 & 1 \\
\hline 5 & 2.7280 & 2.7280 & 1 & 6 & 2.7500 & 2.7600 & 1 \\
\hline 5 & 2.7260 & 2.7300 & 1 & 6 & 2.7500 & 2.7500 & 1 \\
\hline 5 & 2.7310 & 2.7310 & 1 & 6 & 2.7600 & 2.7300 & 1 \\
\hline 5 & 2.7320 & 2.7320 & 1 & 6 & 2.7500 & 2.7400 & 1 \\
\hline 5 & 2.7280 & 2.7330 & 1 & 6 & 2.7500 & 2.7500 & 1 \\
\hline 5 & 2.7320 & 2.7340 & 1 & 6 & 2.7400 & 2.7500 & 1 \\
\hline 6 & 2.7400 & 2.7300 & 1 & 7 & 2.7200 & 2.7500 & 1 \\
\hline 6 & 2.7300 & 2.7300 & 1 & 7 & 2.7200 & 2.7400 & 1 \\
\hline 6 & 2.7300 & 2.7400 & 1 & 7 & 2.7300 & 2.7200 & 1 \\
\hline 6 & 2.7300 & 2.7400 & 1 & 7 & 2.7200 & 2.7400 & 1 \\
\hline 6 & 2.7300 & 2.7400 & 1 & 7 & 2.7200 & 2.7300 & 1 \\
\hline 6 & 2.7400 & 2.7400 & 1 & 7 & 2.7300 & 2.7300 & 1 \\
\hline 7 & 2.6800 & 2.6800 & 1 & 8 & 2.8500 & 2.8600 & 1 \\
\hline 7 & 2.6800 & 2.6900 & 1 & 8 & 2.8300 & 2.8300 & 1 \\
\hline 7 & 2.6900 & 2.6900 & 1 & 8 & 2.8400 & 2.8300 & 1 \\
\hline 7 & 2.6900 & 2.6900 & 1 & 8 & 2.8300 & 2.8300 & 1 \\
\hline 7 & 2.7000 & 2.7000 & 1 & 8 & 2.8300 & 2.8300 & 1 \\
\hline 7 & 2.7000 & 2.7000 & 1 & 8 & 2.8100 & 2.8100 & 1 \\
\hline 8 & 2.7340 & 2.7370 & 1 & 9 & 2.8200 & 2.8100 & 1 \\
\hline 8 & 2.7410 & 2.7370 & 1 & 9 & 2.7700 & 2.7700 & 1 \\
\hline 8 & 2.7420 & 2.7430 & 1 & 9 & 2.7600 & 2.7700 & 1 \\
\hline 8 & 2.7290 & 2.7440 & 1 & 9 & 2.8300 & 2.8100 & 1 \\
\hline
\end{tabular}




\begin{tabular}{|c|c|c|c|c|c|c|}
\hline 8 & 2.7470 & 2.7460 & 1 & 9 & 2.7600 & 2.7500 \\
\hline 8 & 2.7410 & 2.7470 & 1 & 9 & 2.7600 & 2.7400 \\
\hline 9 & 2.7800 & 2.7800 & 1 & 10 & 2.8100 & 2.8100 \\
\hline 9 & 2.7800 & 2.7800 & 1 & 10 & 2.8200 & 2.8200 \\
\hline 9 & 2.7900 & 2.7800 & 1 & 10 & 2.8100 & 2.8100 \\
\hline 9 & 2.7900 & 2.7900 & 1 & 10 & 2.8000 & 2.8000 \\
\hline 9 & 2.7900 & 2.7900 & 1 & 10 & 2.8000 & 2.8000 \\
\hline 9 & 2.7800 & 2.7900 & 1 & 10 & 2.8000 & 2.8000 \\
\hline 10 & 2.6800 & 2.6800 & 1 & 11 & 2.7971 & 2.8050 \\
\hline 10 & 2.6800 & 2.6800 & 1 & 11 & 2.7821 & 2.7904 \\
\hline 10 & 2.6800 & 2.6800 & 1 & 11 & 2.7969 & 2.8003 \\
\hline 10 & 2.6700 & 2.6900 & 1 & 11 & 2.7950 & 2.8052 \\
\hline 10 & 2.7500 & 2.6900 & 1 & 11 & 2.7789 & 2.7808 \\
\hline 10 & 2.6800 & 2.7000 & 1 & 11 & 2.7819 & 2.7852 \\
\hline 11 & 2.7300 & 2.7200 & 1 & 12 & 2.6880 & 2.6780 \\
\hline 11 & 2.7400 & 2.7300 & 1 & 12 & 2.6750 & 2.6710 \\
\hline 11 & 2.7400 & 2.7300 & 1 & 12 & 2.6730 & 2.6810 \\
\hline 11 & 2.7300 & 2.7400 & 1 & 12 & 2.6630 & 2.6860 \\
\hline 11 & 2.7400 & 2.7500 & 1 & 12 & 2.6880 & 2.6810 \\
\hline 11 & 2.7500 & 2.7500 & 1 & 12 & 2.6720 & 2.6760 \\
\hline 12 & 2.7610 & 2.7630 & 1 & 13 & 2.7400 & 2.7370 \\
\hline 12 & 2.7610 & 2.7740 & 1 & 13 & 2.7270 & 2.7350 \\
\hline 12 & 2.7730 & 2.7780 & 1 & 13 & 2.7230 & 2.7220 \\
\hline 12 & 2.7840 & 2.7820 & 1 & 13 & 2.7350 & 2.7340 \\
\hline 12 & 2.7770 & 2.7880 & 1 & 13 & 2.7240 & 2.7150 \\
\hline 12 & 2.7940 & 2.7890 & 1 & 13 & 2.7130 & 2.7120 \\
\hline 13 & 2.7200 & 2.7200 & 1 & 14 & 2.7490 & 2.7500 \\
\hline 13 & 2.7200 & 2.7200 & 1 & 14 & 2.7920 & 2.7940 \\
\hline 13 & 2.7200 & 2.7300 & 1 & 14 & 2.7740 & 2.7720 \\
\hline 13 & 2.7400 & 2.7300 & 1 & 14 & 2.7800 & 2.7850 \\
\hline 13 & 2.7400 & 2.7400 & 1 & 14 & 2.7540 & 2.7610 \\
\hline 13 & 2.7300 & 2.7400 & 1 & 14 & 2.7620 & 2.7610 \\
\hline 14 & 2.7900 & 2.7800 & 1 & 15 & 2.7100 & 2.7200 \\
\hline 14 & 2.7800 & 2.7800 & 1 & 15 & 2.7200 & 2.7200 \\
\hline 14 & 2.7900 & 2.7900 & 1 & 15 & 2.7300 & 2.7300 \\
\hline 14 & 2.7900 & 2.7900 & 1 & 15 & 2.7200 & 2.7300 \\
\hline 14 & 2.7900 & 2.7900 & 1 & 15 & 2.7200 & 2.7200 \\
\hline 14 & 2.7900 & 2.7900 & 1 & 15 & 2.7200 & 2.7100 \\
\hline 15 & 2.7200 & 2.7200 & 1 & 16 & 2.7900 & 2.7900 \\
\hline 15 & 2.7300 & 2.7300 & 1 & 16 & 2.7800 & 2.7900 \\
\hline 15 & 2.7300 & 2.7300 & 1 & 16 & 2.7800 & 2.7900 \\
\hline 15 & 2.7300 & 2.7300 & 1 & 16 & 2.7800 & 2.7900 \\
\hline 15 & 2.7300 & 2.7400 & 1 & 16 & 2.7800 & 2.7700 \\
\hline 15 & 2.7300 & 2.7400 & 1 & 16 & 2.7800 & 2.7800 \\
\hline 1 & 4.2120 & 4.2050 & 2 & 17 & 2.7385 & 2.7390 \\
\hline 1 & 4.2230 & 4.2110 & 2 & 17 & 2.6974 & 2.6996 \\
\hline 1 & 4.2350 & 4.2150 & 2 & 17 & 2.6932 & 2.6922 \\
\hline 1 & 4.2220 & 4.2190 & 2 & 17 & 2.7116 & 2.7109 \\
\hline 1 & 4.2160 & 4.2210 & 2 & 17 & 2.6964 & 2.6942 \\
\hline
\end{tabular}




\begin{tabular}{|c|c|c|c|c|c|c|}
\hline 1 & 4.2270 & 4.2330 & 2 & 17 & 2.7027 & 2.7028 \\
\hline 2 & 4.1860 & 4.1690 & 2 & 18 & 2.7350 & 2.7360 \\
\hline 2 & 4.2580 & 4.2340 & 2 & 18 & 2.7460 & 2.7470 \\
\hline 2 & 4.2420 & 4.2360 & 2 & 18 & 2.7370 & 2.7410 \\
\hline 2 & 4.2510 & 4.2380 & 2 & 18 & 2.7620 & 2.7610 \\
\hline 2 & 4.2460 & 4.2390 & 2 & 18 & 2.7460 & 2.7450 \\
\hline 2 & 4.2350 & 4.2450 & 2 & 18 & 2.7550 & 2.7580 \\
\hline 3 & 4.2460 & 4.2390 & 2 & 19 & 2.7000 & 2.7000 \\
\hline 3 & 4.2420 & 4.2430 & 2 & 19 & 2.7000 & 2.7000 \\
\hline 3 & 4.2440 & 4.2440 & 2 & 19 & 2.7000 & 2.7000 \\
\hline 3 & 4.2460 & 4.2460 & 2 & 19 & 2.7400 & 2.7400 \\
\hline 3 & 4.2450 & 4.2470 & 2 & 19 & 2.7200 & 2.7200 \\
\hline 3 & 4.2530 & 4.2500 & 2 & 19 & 2.7100 & 2.7100 \\
\hline 4 & 4.2100 & 4.2000 & 2 & 20 & 2.7900 & 2.7900 \\
\hline 4 & 4.2100 & 4.2100 & 2 & 20 & 2.7900 & 2.7900 \\
\hline 4 & 4.2200 & 4.2200 & 2 & 20 & 2.8000 & 2.8000 \\
\hline 4 & 4.2200 & 4.2200 & 2 & 20 & 2.8000 & 2.8000 \\
\hline 4 & 4.2100 & 4.2200 & 2 & 20 & 2.8000 & 2.8000 \\
\hline 4 & 4.2300 & 4.2200 & 2 & 20 & 2.8000 & 2.7900 \\
\hline 5 & 4.2310 & 4.2370 & 2 & 21 & 2.7553 & 2.7423 \\
\hline 5 & 4.2400 & 4.2380 & 2 & 21 & 2.7504 & 2.7442 \\
\hline 5 & 4.2400 & 4.2380 & 2 & 21 & 2.7499 & 2.7481 \\
\hline 5 & 4.2440 & 4.2380 & 2 & 21 & 2.7396 & 2.7409 \\
\hline 5 & 4.2380 & 4.2400 & 2 & 21 & 2.7565 & 2.7515 \\
\hline 5 & 4.2460 & 4.2400 & 2 & 21 & 2.7564 & 2.7522 \\
\hline 6 & 4.2500 & 4.2400 & 2 & 22 & 2.7200 & 2.7200 \\
\hline 6 & 4.2500 & 4.2400 & 2 & 22 & 2.7100 & 2.7100 \\
\hline 6 & 4.2600 & 4.2500 & 2 & 22 & 2.7100 & 2.7100 \\
\hline 6 & 4.2500 & 4.2500 & 2 & 22 & 2.7100 & 2.7100 \\
\hline 6 & 4.2400 & 4.2500 & 2 & 22 & 2.7100 & 2.7100 \\
\hline 6 & 4.2600 & 4.2600 & 2 & 22 & 2.7100 & 2.7100 \\
\hline 7 & 4.1500 & 4.1500 & 2 & 23 & 2.7100 & 2.7100 \\
\hline 7 & 4.1600 & 4.1500 & 2 & 23 & 2.7200 & 2.7200 \\
\hline 7 & 4.1700 & 4.1700 & 2 & 23 & 2.7100 & 2.7200 \\
\hline 7 & 4.1800 & 4.1700 & 2 & 23 & 2.7000 & 2.7100 \\
\hline 7 & 4.1700 & 4.1700 & 2 & 23 & 2.7100 & 2.7200 \\
\hline 7 & 4.1800 & 4.1900 & 2 & 23 & 2.7100 & 2.7100 \\
\hline 8 & 4.2390 & 4.2350 & 2 & 24 & 2.8000 & 2.8000 \\
\hline 8 & 4.2460 & 4.2410 & 2 & 24 & 2.7900 & 2.8000 \\
\hline 8 & 4.2350 & 4.2420 & 2 & 24 & 2.8000 & 2.8000 \\
\hline 8 & 4.2490 & 4.2490 & 2 & 24 & 2.8100 & 2.8100 \\
\hline 8 & 4.2430 & 4.2500 & 2 & 24 & 2.8100 & 2.8100 \\
\hline 8 & 4.2330 & 4.2530 & 2 & 24 & 2.7900 & 2.7900 \\
\hline 9 & 4.3000 & 4.2900 & 2 & 1 & 4.2400 & 4.2300 \\
\hline 9 & 4.3000 & 4.2900 & 2 & 1 & 4.2700 & 4.2700 \\
\hline 9 & 4.3000 & 4.3000 & 2 & 1 & 4.2600 & 4.2700 \\
\hline 9 & 4.3100 & 4.3000 & 2 & 1 & 4.2400 & 4.2500 \\
\hline 9 & 4.2900 & 4.3000 & 2 & 1 & 4.2700 & 4.2700 \\
\hline 9 & 4.3000 & 4.3100 & 2 & 1 & 4.2600 & 4.2700 \\
\hline
\end{tabular}




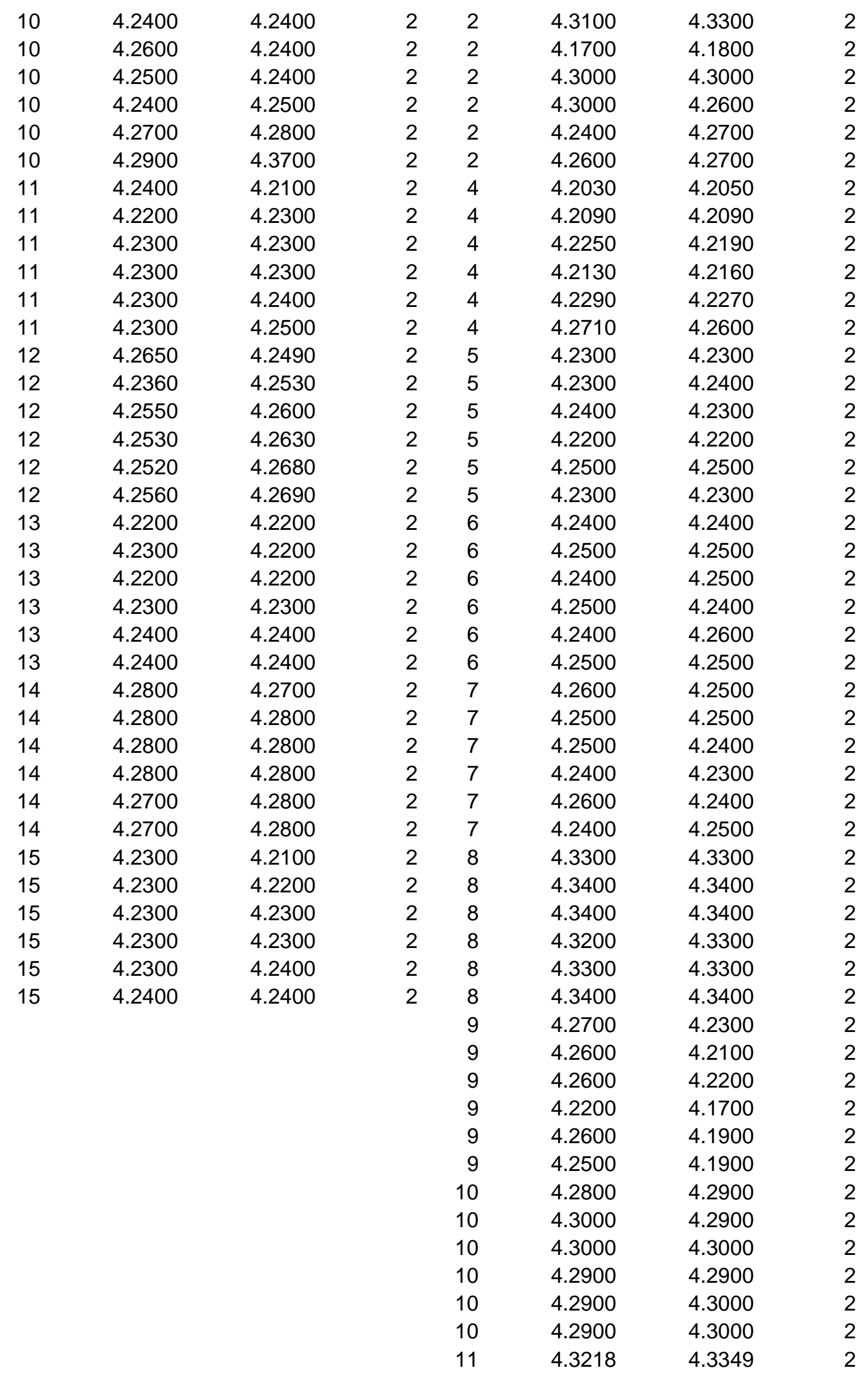




$\begin{array}{llll}11 & 4.3297 & 4.3191 & 2 \\ 11 & 4.3137 & 4.3218 & 2 \\ 11 & 4.2969 & 4.3006 & 2 \\ 11 & 4.3187 & 4.3126 & 2 \\ 11 & 4.3149 & 4.3175 & 2 \\ 12 & 4.1990 & 4.2140 & 2 \\ 12 & 4.1950 & 4.1900 & 2 \\ 12 & 4.2070 & 4.2070 & 2 \\ 12 & 4.1980 & 4.1840 & 2 \\ 12 & 4.1880 & 4.1990 & 2 \\ 12 & 4.2000 & 4.2100 & 2 \\ 13 & 4.2010 & 4.2180 & 2 \\ 13 & 4.2140 & 4.2210 & 2 \\ 13 & 4.2240 & 4.2170 & 2 \\ 13 & 4.1860 & 4.1860 & 2 \\ 13 & 4.2130 & 4.2170 & 2 \\ 13 & 4.1950 & 4.2130 & 2 \\ 14 & 4.1950 & 4.1930 & 2 \\ 14 & 4.1880 & 4.1830 & 2 \\ 14 & 4.1960 & 4.1960 & 2 \\ 14 & 4.1920 & 4.2000 & 2 \\ 14 & 4.2060 & 4.2010 & 2 \\ 14 & 4.2150 & 4.2270 & 2 \\ 15 & 4.2300 & 4.2200 & 2 \\ 15 & 4.2700 & 4.2500 & 2 \\ 15 & 4.2800 & 4.2700 & 2 \\ 15 & 4.2300 & 4.2300 & 2 \\ 15 & 4.2800 & 4.2600 & 2 \\ 15 & 4.2700 & 4.2600 & 2 \\ 16 & 4.2800 & 4.2900 & 2 \\ 16 & 4.2800 & 4.2800 & 2 \\ 16 & 4.2900 & 4.3000 & 2 \\ 16 & 4.2500 & 4.2600 & 2 \\ 16 & 4.2700 & 4.2700 & 2 \\ 16 & 4.2600 & 4.2700 & 2 \\ 17 & 4.2259 & 4.2277 & 2 \\ 17 & 4.1953 & 4.1967 & 2 \\ 17 & 4.1866 & 4.1860 & 2 \\ 17 & 4.2004 & 4.2102 & 2 \\ 17 & 4.2093 & 4.2066 & 2 \\ 17 & 4.2099 & 4.2101 & 2 \\ 18 & 4.3000 & 4.3130 & 2 \\ 18 & 4.2970 & 4.3040 & 2 \\ 18 & 4.3900 & 4.3910 & 2 \\ 18 & 4.3910 & 4.3870 & 2 \\ 18 & 4.3590 & 4.3520 & 2 \\ 18 & 4.3050 & 4.2920 & 2 \\ 19 & 4.2000 & 4.2000 & 2 \\ 19 & 4.2100 & 4.2100 & 2\end{array}$




$\begin{array}{llll}19 & 4.2000 & 4.2000 & 2 \\ 19 & 4.2100 & 4.2100 & 2 \\ 19 & 4.2100 & 4.2100 & 2 \\ 19 & 4.2100 & 4.2200 & 2 \\ 20 & 4.2700 & 4.2700 & 2 \\ 20 & 4.2600 & 4.2600 & 2 \\ 20 & 4.2700 & 4.2700 & 2 \\ 20 & 4.2700 & 4.2700 & 2 \\ 20 & 4.2700 & 4.2700 & 2 \\ 20 & 4.2700 & 4.2700 & 2 \\ 21 & 4.2652 & 4.2689 & 2 \\ 21 & 4.2652 & 4.2689 & 2 \\ 21 & 4.2692 & 4.2667 & 2 \\ 21 & 4.2654 & 4.2678 & 2 \\ 21 & 4.2654 & 4.2678 & 2 \\ 21 & 4.2600 & 4.2580 & 2 \\ 22 & 4.2200 & 4.2100 & 2 \\ 22 & 4.2100 & 4.2200 & 2 \\ 22 & 4.2100 & 4.2200 & 2 \\ 22 & 4.2100 & 4.2100 & 2 \\ 22 & 4.2100 & 4.2200 & 2 \\ 22 & 4.2000 & 4.2100 & 2 \\ 23 & 4.2200 & 4.2100 & 2 \\ 23 & 4.2200 & 4.2200 & 2 \\ 23 & 4.1900 & 4.2000 & 2 \\ 23 & 4.2000 & 4.2000 & 2 \\ 23 & 4.2100 & 4.2100 & 2 \\ 23 & 4.2000 & 4.2000 & 2 \\ 24 & 4.2100 & 4.2300 & 2 \\ 24 & 4.2400 & 4.2200 & 2 \\ 24 & 4.2100 & 4.2100 & 2 \\ 24 & 4.2200 & 4.2200 & 2 \\ 24 & 4.2100 & 4.2100 & 2 \\ 24 & 4.2200 & 4.2100 & 2\end{array}$




\begin{tabular}{|c|c|c|c|c|c|c|c|}
\hline \multicolumn{4}{|c|}{$\mathrm{Fe}_{2} \mathrm{O}_{3}:$ Glass Preparation } & \multicolumn{4}{|c|}{$\mathrm{Fe}_{2} \mathrm{O}_{3}:$ Powder Preparation } \\
\hline Lab & Duplicate 1 & Duplicate 2 & Cement & Lab & Duplicate 1 & Duplicate 2 & Cement \\
\hline 1 & 4.913 & 4.916 & 1 & 1 & 4.720 & 4.780 & 1 \\
\hline 1 & 4.895 & 4.922 & 1 & 1 & 4.820 & 4.810 & 1 \\
\hline 1 & 4.944 & 4.924 & 1 & 1 & 4.790 & 4.790 & 1 \\
\hline 1 & 4.921 & 4.918 & 1 & 1 & 4.680 & 4.740 & 1 \\
\hline 1 & 4.953 & 4.987 & 1 & 1 & 4.770 & 4.760 & 1 \\
\hline 1 & 4.953 & 4.932 & 1 & 1 & 4.720 & 4.680 & 1 \\
\hline 2 & 5.030 & 5.026 & 1 & 2 & 4.730 & 4.750 & 1 \\
\hline 2 & 5.016 & 5.009 & 1 & 2 & 4.740 & 4.750 & 1 \\
\hline 2 & 5.013 & 5.012 & 1 & 2 & 4.750 & 4.750 & 1 \\
\hline 2 & 5.041 & 5.023 & 1 & 2 & 4.720 & 4.730 & 1 \\
\hline 2 & 5.036 & 5.026 & 1 & 2 & 4.750 & 4.720 & 1 \\
\hline 2 & 5.020 & 5.016 & 1 & 2 & 4.700 & 4.700 & 1 \\
\hline 3 & 4.980 & 4.980 & 1 & 3 & 4.735 & 4.764 & 1 \\
\hline 3 & 4.960 & 4.980 & 1 & 3 & 4.731 & 4.732 & 1 \\
\hline 3 & 4.970 & 4.970 & 1 & 3 & 4.705 & 4.723 & 1 \\
\hline 3 & 4.960 & 4.970 & 1 & 3 & 4.749 & 4.754 & 1 \\
\hline 3 & 4.980 & 4.980 & 1 & 3 & 4.741 & 4.734 & 1 \\
\hline 3 & 4.960 & 4.980 & 1 & 3 & 4.667 & 4.647 & 1 \\
\hline 4 & 4.973 & 4.967 & 1 & 4 & 4.803 & 4.797 & 1 \\
\hline 4 & 4.957 & 4.955 & 1 & 4 & 4.899 & 4.908 & 1 \\
\hline 4 & 4.977 & 4.979 & 1 & 4 & 4.862 & 4.867 & 1 \\
\hline 4 & 4.996 & 4.968 & 1 & 4 & 4.820 & 4.829 & 1 \\
\hline 4 & 4.971 & 4.969 & 1 & 4 & 4.873 & 4.885 & 1 \\
\hline 4 & 4.992 & 4.986 & 1 & 4 & 4.827 & 4.831 & 1 \\
\hline 5 & 4.970 & 4.950 & 1 & 5 & 4.960 & 4.950 & 1 \\
\hline 5 & 4.960 & 4.950 & 1 & 5 & 4.940 & 4.940 & 1 \\
\hline 5 & 4.940 & 4.980 & 1 & 5 & 4.930 & 4.930 & 1 \\
\hline 5 & 5.020 & 4.990 & 1 & 5 & 4.960 & 4.960 & 1 \\
\hline 5 & 4.980 & 5.000 & 1 & 5 & 4.940 & 4.940 & 1 \\
\hline 5 & 4.970 & 4.980 & 1 & 5 & 4.960 & 4.960 & 1 \\
\hline 6 & 5.130 & 5.140 & 1 & 6 & 4.850 & 4.860 & 1 \\
\hline 6 & 5.150 & 5.150 & 1 & 6 & 4.830 & 4.860 & 1 \\
\hline 6 & 5.140 & 5.120 & 1 & 6 & 4.840 & 4.850 & 1 \\
\hline 6 & 5.130 & 5.120 & 1 & 6 & 4.800 & 4.820 & 1 \\
\hline 6 & 5.140 & 5.150 & 1 & 6 & 4.810 & 4.810 & 1 \\
\hline 6 & 5.130 & 5.130 & 1 & 6 & 4.830 & 4.810 & 1 \\
\hline 7 & 5.002 & 4.979 & 1 & 7 & 4.790 & 4.800 & 1 \\
\hline 7 & 5.032 & 5.027 & 1 & 7 & 4.790 & 4.800 & 1 \\
\hline 7 & 5.017 & 5.006 & 1 & 7 & 4.780 & 4.790 & 1 \\
\hline 7 & 4.979 & 4.986 & 1 & 7 & 4.810 & 4.800 & 1 \\
\hline 7 & 4.973 & 4.973 & 1 & 7 & 4.780 & 4.780 & 1 \\
\hline 7 & 4.998 & 5.000 & 1 & 7 & 4.800 & 4.790 & 1 \\
\hline 8 & 5.020 & 5.010 & 1 & 8 & 4.990 & 4.980 & 1 \\
\hline 8 & 5.030 & 4.980 & 1 & 8 & 5.000 & 5.000 & 1 \\
\hline 8 & 5.010 & 5.020 & 1 & 8 & 4.990 & 5.000 & 1 \\
\hline 8 & 4.990 & 5.010 & 1 & 8 & 4.990 & 4.970 & 1 \\
\hline
\end{tabular}




\begin{tabular}{|c|c|c|c|c|c|c|}
\hline 8 & 5.030 & 5.000 & 1 & 8 & 4.960 & 4.980 \\
\hline 8 & 5.000 & 5.010 & 1 & 8 & 4.980 & 5.000 \\
\hline 9 & 4.990 & 4.980 & 1 & 9 & 4.820 & 4.830 \\
\hline 9 & 5.000 & 4.980 & 1 & 9 & 4.830 & 4.840 \\
\hline 9 & 4.950 & 4.940 & 1 & 9 & 4.820 & 4.820 \\
\hline 9 & 4.940 & 4.930 & 1 & 9 & 4.840 & 4.840 \\
\hline 9 & 4.990 & 4.960 & 1 & 9 & 4.820 & 4.830 \\
\hline 9 & 4.940 & 4.950 & 1 & 9 & 4.830 & 4.840 \\
\hline 10 & 4.930 & 4.970 & 1 & 10 & 4.830 & 4.840 \\
\hline 10 & 4.940 & 4.910 & 1 & 10 & 4.860 & 4.860 \\
\hline 10 & 4.980 & 4.940 & 1 & 10 & 4.820 & 4.830 \\
\hline 10 & 4.980 & 4.970 & 1 & 10 & 4.830 & 4.840 \\
\hline 10 & 4.920 & 4.970 & 1 & 10 & 4.860 & 4.880 \\
\hline 10 & 4.920 & 4.930 & 1 & 10 & 4.840 & 4.830 \\
\hline 11 & 4.990 & 5.000 & 1 & 11 & 4.884 & 4.887 \\
\hline 11 & 5.000 & 4.990 & 1 & 11 & 4.880 & 4.888 \\
\hline 11 & 5.000 & 4.980 & 1 & 11 & 4.892 & 4.890 \\
\hline 11 & 5.050 & 5.050 & 1 & 11 & 4.895 & 4.903 \\
\hline 11 & 5.010 & 5.050 & 1 & 11 & 4.881 & 4.877 \\
\hline 11 & 5.020 & 5.000 & 1 & 11 & 4.880 & 4.882 \\
\hline 12 & 5.019 & 5.043 & 1 & 13 & 4.748 & 4.789 \\
\hline 12 & 5.044 & 5.080 & 1 & 13 & 4.751 & 4.763 \\
\hline 12 & 5.036 & 5.088 & 1 & 13 & 4.761 & 4.752 \\
\hline 12 & 5.059 & 5.088 & 1 & 13 & 4.768 & 4.769 \\
\hline 12 & 5.122 & 5.105 & 1 & 13 & 4.769 & 4.770 \\
\hline 12 & 5.103 & 5.079 & 1 & 13 & 4.791 & 4.754 \\
\hline 13 & 4.950 & 5.000 & 1 & 14 & 4.740 & 4.750 \\
\hline 13 & 4.980 & 5.020 & 1 & 14 & 4.780 & 4.760 \\
\hline 13 & 5.010 & 5.000 & 1 & 14 & 4.800 & 4.770 \\
\hline 13 & 4.990 & 5.010 & 1 & 14 & 4.810 & 4.800 \\
\hline 13 & 4.980 & 5.020 & 1 & 14 & 4.790 & 4.800 \\
\hline 13 & 5.020 & 5.030 & 1 & 14 & 4.800 & 4.790 \\
\hline 14 & 5.030 & 5.030 & 1 & 15 & 4.743 & 4.798 \\
\hline 14 & 5.020 & 5.020 & 1 & 15 & 4.743 & 4.791 \\
\hline 14 & 5.010 & 5.000 & 1 & 15 & 4.767 & 4.794 \\
\hline 14 & 5.000 & 5.000 & 1 & 15 & 4.729 & 4.800 \\
\hline 14 & 5.010 & 5.030 & 1 & 15 & 4.770 & 4.776 \\
\hline 14 & 5.020 & 5.040 & 1 & 15 & 4.764 & 4.791 \\
\hline 15 & 5.100 & 5.100 & 1 & 16 & 4.906 & 4.913 \\
\hline 15 & 5.050 & 5.040 & 1 & 16 & 4.960 & 4.963 \\
\hline 15 & 5.040 & 5.040 & 1 & 16 & 4.931 & 4.940 \\
\hline 15 & 5.040 & 5.040 & 1 & 16 & 5.027 & 5.031 \\
\hline 15 & 5.030 & 5.040 & 1 & 16 & 4.956 & 4.960 \\
\hline 15 & 5.010 & 5.020 & 1 & 16 & 4.953 & 4.948 \\
\hline 16 & 5.048 & 5.026 & 1 & 17 & 4.810 & 4.820 \\
\hline 16 & 5.044 & 5.039 & 1 & 17 & 4.800 & 4.780 \\
\hline 16 & 5.048 & 5.052 & 1 & 17 & 4.780 & 4.780 \\
\hline 16 & 5.058 & 5.043 & 1 & 17 & 4.760 & 4.800 \\
\hline 16 & 5.048 & 5.039 & 1 & 17 & 4.760 & 4.760 \\
\hline
\end{tabular}




\begin{tabular}{|c|c|c|c|c|c|c|}
\hline 16 & 5.049 & 5.027 & 1 & 17 & 4.760 & 4.790 \\
\hline 17 & 5.010 & 5.020 & 1 & 18 & 4.830 & 4.850 \\
\hline 17 & 5.020 & 5.010 & 1 & 18 & 4.820 & 4.820 \\
\hline 17 & 5.030 & 5.010 & 1 & 18 & 4.840 & 4.840 \\
\hline 17 & 5.020 & 5.010 & 1 & 18 & 4.830 & 4.830 \\
\hline 17 & 5.030 & 5.040 & 1 & 18 & 4.830 & 4.830 \\
\hline 17 & 5.020 & 5.030 & 1 & 18 & 4.820 & 4.830 \\
\hline 1 & 5.047 & 5.030 & 2 & 19 & 4.880 & 4.885 \\
\hline 1 & 5.046 & 5.049 & 2 & 19 & 4.809 & 4.829 \\
\hline 1 & 5.025 & 5.056 & 2 & 19 & 4.822 & 4.825 \\
\hline 1 & 5.064 & 5.070 & 2 & 19 & 4.821 & 4.816 \\
\hline 1 & 5.035 & 5.038 & 2 & 19 & 4.813 & 4.809 \\
\hline 1 & 5.068 & 5.074 & 2 & 19 & 4.823 & 4.824 \\
\hline 2 & 5.145 & 5.126 & 2 & 20 & 4.840 & 4.840 \\
\hline 2 & 5.132 & 5.143 & 2 & 20 & 4.800 & 4.830 \\
\hline 2 & 5.126 & 5.132 & 2 & 20 & 4.770 & 4.780 \\
\hline 2 & 5.149 & 5.146 & 2 & 20 & 4.850 & 4.870 \\
\hline 2 & 5.143 & 5.128 & 2 & 20 & 4.800 & 4.820 \\
\hline 2 & 5.132 & 5.139 & 2 & 20 & 4.790 & 4.800 \\
\hline 3 & 5.120 & 5.110 & 2 & 21 & 4.900 & 4.890 \\
\hline 3 & 5.120 & 5.130 & 2 & 21 & 4.890 & 4.900 \\
\hline 3 & 5.110 & 5.120 & 2 & 21 & 4.860 & 4.850 \\
\hline 3 & 5.120 & 5.120 & 2 & 21 & 4.910 & 4.910 \\
\hline 3 & 5.100 & 5.100 & 2 & 21 & 4.880 & 4.890 \\
\hline 3 & 5.120 & 5.110 & 2 & 21 & 4.880 & 4.880 \\
\hline 4 & 5.091 & 5.095 & 2 & 22 & 4.710 & 4.705 \\
\hline 4 & 5.096 & 5.087 & 2 & 22 & 4.714 & 4.710 \\
\hline 4 & 5.088 & 5.087 & 2 & 22 & 4.710 & 4.709 \\
\hline 4 & 5.109 & 5.102 & 2 & 22 & 4.684 & 4.683 \\
\hline 4 & 5.118 & 5.125 & 2 & 22 & 4.700 & 4.689 \\
\hline 4 & 5.107 & 5.127 & 2 & 22 & 4.702 & 4.694 \\
\hline 5 & 5.080 & 5.070 & 2 & 1 & 4.970 & 4.900 \\
\hline 5 & 5.120 & 5.100 & 2 & 1 & 4.930 & 4.950 \\
\hline 5 & 5.080 & 5.090 & 2 & 1 & 4.960 & 4.950 \\
\hline 5 & 5.150 & 5.140 & 2 & 1 & 4.910 & 4.970 \\
\hline 5 & 5.140 & 5.120 & 2 & 1 & 4.930 & 4.980 \\
\hline 5 & 5.110 & 5.140 & 2 & 1 & 4.960 & 4.940 \\
\hline 6 & 5.250 & 5.240 & 2 & 2 & 5.240 & 5.220 \\
\hline 6 & 5.280 & 5.260 & 2 & 2 & 5.220 & 5.180 \\
\hline 6 & 5.270 & 5.300 & 2 & 2 & 5.220 & 5.220 \\
\hline 6 & 5.230 & 5.270 & 2 & 2 & 5.230 & 5.220 \\
\hline 6 & 5.260 & 5.300 & 2 & 2 & 5.220 & 5.230 \\
\hline 6 & 5.260 & 5.260 & 2 & 2 & 5.220 & 5.200 \\
\hline 7 & 5.141 & 5.123 & 2 & 3 & 5.293 & 5.306 \\
\hline 7 & 5.137 & 5.156 & 2 & 3 & 5.278 & 5.282 \\
\hline 7 & 5.133 & 5.166 & 2 & 3 & 5.301 & 5.290 \\
\hline 7 & 5.138 & 5.145 & 2 & 3 & 5.309 & 5.337 \\
\hline 7 & 5.182 & 5.150 & 2 & 3 & 5.335 & 5.356 \\
\hline 7 & 5.149 & 5.151 & 2 & 3 & 5.324 & 5.323 \\
\hline
\end{tabular}




\begin{tabular}{|c|c|c|c|c|c|c|}
\hline 8 & 5.130 & 5.130 & 2 & 4 & 5.118 & 5.117 \\
\hline 8 & 5.150 & 5.110 & 2 & 4 & 5.127 & 5.121 \\
\hline 8 & 5.140 & 5.140 & 2 & 4 & 5.093 & 5.099 \\
\hline 8 & 5.110 & 5.110 & 2 & 4 & 5.154 & 5.166 \\
\hline 8 & 5.110 & 5.140 & 2 & 4 & 5.119 & 5.120 \\
\hline 8 & 5.100 & 5.120 & 2 & 4 & 5.118 & 5.125 \\
\hline 9 & 5.080 & 5.080 & 2 & 5 & 5.280 & 5.270 \\
\hline 9 & 5.070 & 5.080 & 2 & 5 & 5.280 & 5.270 \\
\hline 9 & 5.130 & 5.090 & 2 & 5 & 5.290 & 5.290 \\
\hline 9 & 5.090 & 5.060 & 2 & 5 & 5.240 & 5.260 \\
\hline 9 & 5.060 & 5.060 & 2 & 5 & 5.280 & 5.280 \\
\hline 9 & 5.130 & 5.070 & 2 & 5 & 5.270 & 5.270 \\
\hline 10 & 5.050 & 5.010 & 2 & 6 & 5.060 & 5.060 \\
\hline 10 & 5.020 & 5.020 & 2 & 6 & 5.060 & 5.070 \\
\hline 10 & 5.000 & 5.000 & 2 & 6 & 5.050 & 5.060 \\
\hline 10 & 4.940 & 5.040 & 2 & 6 & 5.070 & 5.060 \\
\hline 10 & 5.010 & 5.070 & 2 & 6 & 5.070 & 5.090 \\
\hline 10 & 5.050 & 5.040 & 2 & 6 & 5.080 & 5.050 \\
\hline 11 & 5.100 & 5.120 & 2 & 7 & 5.020 & 5.030 \\
\hline 11 & 5.140 & 5.120 & 2 & 7 & 5.020 & 5.030 \\
\hline 11 & 5.130 & 5.100 & 2 & 7 & 5.040 & 5.020 \\
\hline 11 & 5.120 & 5.130 & 2 & 7 & 5.020 & 5.010 \\
\hline 11 & 5.130 & 5.140 & 2 & 7 & 5.020 & 5.020 \\
\hline 11 & 5.150 & 5.110 & 2 & 7 & 5.040 & 5.040 \\
\hline 12 & 5.162 & 5.180 & 2 & 8 & 5.290 & 5.260 \\
\hline 12 & 5.135 & 5.203 & 2 & 8 & 5.270 & 5.280 \\
\hline 12 & 5.191 & 5.245 & 2 & 8 & 5.290 & 5.290 \\
\hline 12 & 5.200 & 5.163 & 2 & 8 & 5.260 & 5.270 \\
\hline 12 & 5.175 & 5.183 & 2 & 8 & 5.310 & 5.300 \\
\hline 12 & 5.185 & 5.197 & 2 & 8 & 5.280 & 5.270 \\
\hline 13 & 5.090 & 5.070 & 2 & 9 & 5.160 & 5.150 \\
\hline 13 & 5.120 & 5.110 & 2 & 9 & 5.130 & 5.150 \\
\hline 13 & 5.120 & 5.150 & 2 & 9 & 5.120 & 5.120 \\
\hline 13 & 5.020 & 5.060 & 2 & 9 & 5.140 & 5.140 \\
\hline 13 & 5.080 & 5.090 & 2 & 9 & 5.130 & 5.140 \\
\hline 13 & 5.120 & 5.150 & 2 & 9 & 5.140 & 5.140 \\
\hline 14 & 5.140 & 5.150 & 2 & 10 & 5.220 & 5.210 \\
\hline 14 & 5.130 & 5.150 & 2 & 10 & 5.170 & 5.170 \\
\hline 14 & 5.120 & 5.120 & 2 & 10 & 5.220 & 5.220 \\
\hline 14 & 5.140 & 5.140 & 2 & 10 & 5.170 & 5.160 \\
\hline 14 & 5.180 & 5.210 & 2 & 10 & 5.210 & 5.220 \\
\hline 14 & 5.150 & 5.160 & 2 & 10 & 5.140 & 5.140 \\
\hline 15 & 5.200 & 5.210 & 2 & 11 & 5.099 & 5.087 \\
\hline 15 & 5.240 & 5.230 & 2 & 11 & 5.088 & 5.095 \\
\hline 15 & 5.220 & 5.210 & 2 & 11 & 5.082 & 5.078 \\
\hline 15 & 5.190 & 5.210 & 2 & 11 & 5.020 & 5.036 \\
\hline 15 & 5.190 & 5.180 & 2 & 11 & 5.063 & 5.066 \\
\hline 15 & 5.200 & 5.210 & 2 & 11 & 5.085 & 5.068 \\
\hline 16 & 5.169 & 5.164 & 2 & 13 & 5.292 & 5.303 \\
\hline
\end{tabular}




\begin{tabular}{|c|c|c|c|c|c|}
\hline 5.169 & 5.164 & 2 & 13 & 5.327 & 5.314 \\
\hline 5.166 & 5.162 & 2 & 13 & 5.320 & 5.285 \\
\hline 5.172 & 5.165 & 2 & 13 & 5.311 & 5.310 \\
\hline 5.172 & 5.165 & 2 & 13 & 5.319 & 5.318 \\
\hline 5.173 & 5.161 & 2 & 13 & 5.305 & 5.301 \\
\hline 5.140 & 5.130 & 2 & 14 & 5.020 & 5.030 \\
\hline 5.120 & 5.130 & 2 & 14 & 5.050 & 5.050 \\
\hline 5.140 & 5.150 & 2 & 14 & 5.070 & 5.070 \\
\hline 5.160 & 5.170 & 2 & 14 & 5.030 & 5.010 \\
\hline 5.160 & 5.160 & 2 & 14 & 5.020 & 5.030 \\
\hline \multirow[t]{39}{*}{5.160} & 5.150 & 2 & 14 & 5.040 & 5.030 \\
\hline & & & 15 & 4.969 & 5.008 \\
\hline & & & 15 & 4.976 & 5.029 \\
\hline & & & 15 & 5.010 & 5.039 \\
\hline & & & 15 & 4.947 & 5.040 \\
\hline & & & 15 & 5.011 & 5.056 \\
\hline & & & 15 & 5.005 & 5.018 \\
\hline & & & 16 & 5.210 & 5.203 \\
\hline & & & 16 & 5.197 & 5.211 \\
\hline & & & 16 & 5.217 & 5.217 \\
\hline & & & 16 & 5.221 & 5.211 \\
\hline & & & 16 & 5.204 & 5.200 \\
\hline & & & 16 & 5.233 & 5.231 \\
\hline & & & 17 & 5.160 & 5.130 \\
\hline & & & 17 & 5.150 & 5.160 \\
\hline & & & 17 & 5.140 & 5.140 \\
\hline & & & 17 & 5.120 & 5.120 \\
\hline & & & 17 & 5.140 & 5.110 \\
\hline & & & 17 & 5.110 & 5.140 \\
\hline & & & 18 & 5.050 & 5.040 \\
\hline & & & 18 & 5.060 & 5.050 \\
\hline & & & 18 & 5.060 & 5.060 \\
\hline & & & 18 & 5.010 & 5.020 \\
\hline & & & 18 & 5.020 & 5.010 \\
\hline & & & 18 & 5.030 & 5.020 \\
\hline & & & 19 & 5.097 & 5.114 \\
\hline & & & 19 & 5.047 & 5.046 \\
\hline & & & 19 & 5.096 & 5.137 \\
\hline & & & 19 & 5.081 & 5.089 \\
\hline & & & 19 & 5.070 & 5.064 \\
\hline & & & 19 & 5.071 & 5.076 \\
\hline & & & 20 & 5.060 & 5.100 \\
\hline & & & 20 & 5.070 & 5.100 \\
\hline & & & 20 & 5.070 & 5.110 \\
\hline & & & 20 & 5.070 & 5.090 \\
\hline & & & 20 & 5.100 & 5.110 \\
\hline & & & 20 & 5.070 & 5.100 \\
\hline & & & 21 & 5.170 & 5.170 \\
\hline & & & 21 & 5.180 & 5.190 \\
\hline
\end{tabular}




$\begin{array}{llll}21 & 5.180 & 5.190 & 2 \\ 21 & 5.200 & 5.200 & 2 \\ 21 & 5.240 & 5.240 & 2 \\ 21 & 5.160 & 5.160 & 2 \\ 22 & 4.991 & 4.982 & 2 \\ 22 & 4.991 & 4.982 & 2 \\ 22 & 5.018 & 5.019 & 2 \\ 22 & 5.011 & 5.002 & 2 \\ 22 & 5.011 & 5.002 & 2 \\ 22 & 5.011 & 5.010 & 2\end{array}$




\begin{tabular}{|c|c|c|c|c|c|c|c|}
\hline \multicolumn{2}{|c|}{$\begin{array}{l}\text { CaO: Glass } \\
\text { Preparation }\end{array}$} & \multirow[b]{2}{*}{$\begin{array}{l}\text { Duplicate } \\
2\end{array}$} & \multirow[b]{2}{*}{$\begin{array}{l}\text { Cemen } \\
t\end{array}$} & \multicolumn{2}{|c|}{$\begin{array}{l}\text { CaO: Powder } \\
\text { Preparation }\end{array}$} & \multirow[b]{2}{*}{$\begin{array}{l}\text { Duplicate } \\
2\end{array}$} & \multirow[b]{2}{*}{$\begin{array}{l}\text { Cemen } \\
\mathbf{t}\end{array}$} \\
\hline Lab & Duplicate 1 & & & Lab & Duplicate 1 & & \\
\hline 1 & 64.180 & 64.190 & 1 & 1 & 63.877 & 63.818 & 1 \\
\hline 1 & 64.160 & 64.250 & 1 & 1 & 63.903 & 63.923 & 1 \\
\hline 1 & 64.230 & 64.290 & 1 & 1 & 63.814 & 63.789 & 1 \\
\hline 1 & 64.050 & 64.200 & 1 & 1 & 64.052 & 63.994 & 1 \\
\hline 1 & 64.120 & 64.000 & 1 & 1 & 64.061 & 64.155 & 1 \\
\hline 1 & 64.100 & 64.020 & 1 & 1 & 63.871 & 63.883 & 1 \\
\hline 2 & 64.140 & 64.180 & 1 & 2 & 63.384 & 63.391 & 1 \\
\hline 2 & 64.370 & 64.300 & 1 & 2 & 63.764 & 63.777 & 1 \\
\hline 2 & 64.010 & 64.040 & 1 & 2 & 63.680 & 63.692 & 1 \\
\hline 2 & 64.050 & 64.000 & 1 & 2 & 63.659 & 63.654 & 1 \\
\hline 2 & 64.360 & 64.350 & 1 & 2 & 63.410 & 63.374 & 1 \\
\hline 2 & 64.070 & 64.100 & 1 & 2 & 63.526 & 63.514 & 1 \\
\hline 3 & 63.840 & 63.740 & 1 & 3 & 63.910 & 64.120 & 1 \\
\hline 3 & 63.720 & 63.800 & 1 & 3 & 64.190 & 64.230 & 1 \\
\hline 3 & 63.820 & 63.730 & 1 & 3 & 64.260 & 64.350 & 1 \\
\hline 3 & 64.030 & 63.640 & 1 & 3 & 64.070 & 64.090 & 1 \\
\hline 3 & 64.010 & 63.790 & 1 & 3 & 64.220 & 64.170 & 1 \\
\hline 3 & 63.860 & 63.930 & 1 & 3 & 64.230 & 64.290 & 1 \\
\hline 4 & 63.207 & 63.194 & 1 & 4 & 64.210 & 64.230 & 1 \\
\hline 4 & 63.139 & 63.227 & 1 & 4 & 64.140 & 64.140 & 1 \\
\hline 4 & 63.264 & 63.147 & 1 & 4 & 64.120 & 64.160 & 1 \\
\hline 4 & 63.287 & 63.224 & 1 & 4 & 64.250 & 64.230 & 1 \\
\hline 4 & 63.486 & 63.322 & 1 & 4 & 63.990 & 64.030 & 1 \\
\hline 4 & 63.207 & 63.274 & 1 & 4 & 63.700 & 63.940 & 1 \\
\hline 5 & 63.627 & 63.652 & 1 & 5 & 63.880 & 63.960 & 1 \\
\hline 5 & 63.679 & 63.665 & 1 & 5 & 63.900 & 63.960 & 1 \\
\hline 5 & 63.597 & 63.701 & 1 & 5 & 63.910 & 63.980 & 1 \\
\hline 5 & 63.801 & 63.720 & 1 & 5 & 63.920 & 63.930 & 1 \\
\hline 5 & 63.711 & 63.668 & 1 & 5 & 63.790 & 63.820 & 1 \\
\hline 5 & 63.772 & 63.772 & 1 & 5 & 63.900 & 63.950 & 1 \\
\hline 6 & 64.250 & 64.300 & 1 & 6 & 64.150 & 64.230 & 1 \\
\hline 6 & 64.020 & 64.030 & 1 & 6 & 64.180 & 64.270 & 1 \\
\hline 6 & 63.940 & 63.950 & 1 & 6 & 64.190 & 64.270 & 1 \\
\hline 6 & 63.830 & 63.910 & 1 & 6 & 64.360 & 64.420 & 1 \\
\hline 6 & 64.200 & 64.240 & 1 & 6 & 64.190 & 64.300 & 1 \\
\hline 6 & 64.180 & 64.340 & 1 & 6 & 64.250 & 64.310 & 1 \\
\hline 7 & 63.510 & 63.580 & 1 & 7 & 63.550 & 63.950 & 1 \\
\hline 7 & 63.540 & 63.600 & 1 & 7 & 63.880 & 63.910 & 1 \\
\hline 7 & 63.630 & 63.640 & 1 & 7 & 63.810 & 64.020 & 1 \\
\hline 7 & 63.680 & 63.660 & 1 & 7 & 63.640 & 63.710 & 1 \\
\hline 7 & 63.740 & 63.670 & 1 & 7 & 63.840 & 63.810 & 1 \\
\hline 7 & 63.620 & 63.750 & 1 & 7 & 63.780 & 63.980 & 1 \\
\hline 8 & 64.280 & 64.300 & 1 & 8 & 63.850 & 64.040 & 1 \\
\hline 8 & 64.350 & 64.400 & 1 & 8 & 63.930 & 64.000 & 1 \\
\hline
\end{tabular}




\begin{tabular}{|c|c|c|c|c|c|c|}
\hline 8 & 64.350 & 64.310 & 1 & 8 & 63.990 & 64.010 \\
\hline 8 & 64.310 & 64.310 & 1 & 8 & 64.040 & 64.140 \\
\hline 8 & 64.230 & 64.250 & 1 & 8 & 64.130 & 64.240 \\
\hline 8 & 64.280 & 64.260 & 1 & 8 & 64.240 & 64.250 \\
\hline 9 & 63.610 & 63.605 & 1 & 9 & 63.920 & 63.930 \\
\hline 9 & 63.753 & 63.494 & 1 & 9 & 64.200 & 64.130 \\
\hline 9 & 63.706 & 63.441 & 1 & 9 & 64.040 & 64.100 \\
\hline 9 & 63.704 & 63.682 & 1 & 9 & 64.030 & 64.050 \\
\hline 9 & 63.487 & 63.498 & 1 & 9 & 64.010 & 64.090 \\
\hline 9 & 63.517 & 63.462 & 1 & 9 & 64.070 & 64.130 \\
\hline 10 & 63.970 & 63.790 & 1 & 10 & 63.650 & 63.640 \\
\hline 10 & 64.040 & 64.020 & 1 & 10 & 64.010 & 63.840 \\
\hline 10 & 63.900 & 63.950 & 1 & 10 & 64.020 & 64.110 \\
\hline 10 & 64.040 & 64.070 & 1 & 10 & 63.810 & 63.780 \\
\hline 10 & 63.980 & 63.990 & 1 & 10 & 63.850 & 63.840 \\
\hline 10 & 63.990 & 64.080 & 1 & 10 & 63.810 & 63.800 \\
\hline 11 & 63.430 & 63.398 & 1 & 11 & 64.800 & 64.800 \\
\hline 11 & 63.436 & 63.454 & 1 & 11 & 64.710 & 64.700 \\
\hline 11 & 63.188 & 63.441 & 1 & 11 & 64.720 & 64.740 \\
\hline 11 & 63.288 & 63.407 & 1 & 11 & 64.640 & 64.730 \\
\hline 11 & 63.413 & 63.388 & 1 & 11 & 64.680 & 64.650 \\
\hline 11 & 63.483 & 63.243 & 1 & 11 & 64.660 & 64.680 \\
\hline 12 & 64.180 & 64.210 & 1 & 12 & 64.820 & 64.700 \\
\hline 12 & 64.250 & 64.300 & 1 & 12 & 64.790 & 64.790 \\
\hline 12 & 64.240 & 64.240 & 1 & 12 & 64.770 & 64.640 \\
\hline 12 & 63.900 & 63.930 & 1 & 12 & 64.720 & 64.790 \\
\hline 12 & 64.080 & 64.150 & 1 & 12 & 64.790 & 64.920 \\
\hline 12 & 64.050 & 64.060 & 1 & 12 & 64.410 & 64.570 \\
\hline 13 & 63.872 & 63.855 & 1 & 13 & 63.890 & 63.850 \\
\hline 13 & 63.861 & 63.843 & 1 & 13 & 64.100 & 64.110 \\
\hline 13 & 63.906 & 63.881 & 1 & 13 & 64.090 & 64.110 \\
\hline 13 & 63.846 & 63.841 & 1 & 13 & 63.940 & 63.970 \\
\hline 13 & 63.871 & 63.875 & 1 & 13 & 64.040 & 64.070 \\
\hline 13 & 63.912 & 63.893 & 1 & 13 & 64.070 & 64.100 \\
\hline 14 & 64.330 & 64.290 & 1 & 14 & 63.396 & 63.384 \\
\hline 14 & 64.280 & 64.230 & 1 & 14 & 63.597 & 63.611 \\
\hline 14 & 64.190 & 64.250 & 1 & 14 & 63.684 & 63.697 \\
\hline 14 & 64.200 & 64.290 & 1 & 14 & 63.395 & 63.358 \\
\hline 14 & 64.200 & 64.310 & 1 & 14 & 63.523 & 63.561 \\
\hline 14 & 64.410 & 64.290 & 1 & 14 & 63.822 & 63.828 \\
\hline 15 & 63.119 & 63.143 & 1 & 15 & 64.220 & 64.210 \\
\hline 15 & 63.316 & 63.317 & 1 & 15 & 64.290 & 64.270 \\
\hline 15 & 63.301 & 63.319 & 1 & 15 & 64.270 & 64.250 \\
\hline 15 & 63.519 & 63.563 & 1 & 15 & 64.250 & 64.260 \\
\hline 15 & 63.212 & 63.132 & 1 & 15 & 64.270 & 64.340 \\
\hline 15 & 63.197 & 63.162 & 1 & 15 & 64.060 & 64.160 \\
\hline 16 & 64.240 & 64.240 & 1 & 16 & 64.096 & 63.987 \\
\hline 16 & 64.190 & 64.200 & 1 & 16 & 64.188 & 63.992 \\
\hline 16 & 64.310 & 64.240 & 1 & 16 & 64.104 & 64.076 \\
\hline
\end{tabular}




\begin{tabular}{|c|c|c|c|c|c|c|}
\hline 16 & 64.450 & 64.450 & 1 & 16 & 63.851 & 63.896 \\
\hline 16 & 64.350 & 64.370 & 1 & 16 & 64.239 & 64.046 \\
\hline 16 & 64.510 & 64.560 & 1 & 16 & 64.084 & 64.079 \\
\hline 17 & 63.920 & 63.860 & 1 & 17 & 64.110 & 64.200 \\
\hline 17 & 63.930 & 63.900 & 1 & 17 & 64.170 & 64.190 \\
\hline 17 & 63.960 & 64.020 & 1 & 17 & 64.200 & 64.200 \\
\hline 17 & 63.950 & 63.930 & 1 & 17 & 64.170 & 64.190 \\
\hline 17 & 63.840 & 63.810 & 1 & 17 & 64.210 & 64.150 \\
\hline 17 & 63.960 & 63.960 & 1 & 17 & 64.220 & 64.240 \\
\hline 1 & 63.930 & 63.940 & 2 & 18 & 63.269 & 63.302 \\
\hline 1 & 63.850 & 63.840 & 2 & 18 & 63.576 & 63.604 \\
\hline 1 & 63.700 & 63.700 & 2 & 18 & 63.373 & 63.376 \\
\hline 1 & 63.530 & 63.440 & 2 & 18 & 63.460 & 63.483 \\
\hline 1 & 63.620 & 63.500 & 2 & 18 & 63.608 & 63.623 \\
\hline 1 & 63.650 & 63.620 & 2 & 18 & 63.511 & 63.523 \\
\hline 2 & 63.440 & 63.400 & 2 & 19 & 63.800 & 63.790 \\
\hline 2 & 63.680 & 63.680 & 2 & 19 & 63.910 & 63.930 \\
\hline 2 & 63.970 & 63.980 & 2 & 19 & 64.010 & 64.080 \\
\hline 2 & 63.610 & 63.620 & 2 & 19 & 64.030 & 64.090 \\
\hline 2 & 63.990 & 63.960 & 2 & 19 & 64.100 & 64.110 \\
\hline 2 & 63.510 & 63.550 & 2 & 19 & 64.030 & 64.090 \\
\hline 3 & 63.490 & 63.520 & 2 & 20 & 64.765 & 64.798 \\
\hline 3 & 63.410 & 63.470 & 2 & 20 & 64.742 & 64.766 \\
\hline 3 & 63.580 & 63.600 & 2 & 20 & 64.688 & 64.725 \\
\hline 3 & 63.760 & 63.920 & 2 & 20 & 64.815 & 64.902 \\
\hline 3 & 63.710 & 63.540 & 2 & 20 & 64.852 & 64.893 \\
\hline 3 & 63.670 & 63.620 & 2 & 20 & 64.394 & 64.409 \\
\hline 4 & 62.908 & 62.915 & 2 & 21 & 64.760 & 64.760 \\
\hline 4 & 62.974 & 62.619 & 2 & 21 & 64.780 & 64.710 \\
\hline 4 & 62.712 & 62.735 & 2 & 21 & 64.630 & 64.580 \\
\hline 4 & 62.886 & 62.942 & 2 & 21 & 64.570 & 64.670 \\
\hline 4 & 62.812 & 62.870 & 2 & 21 & 64.770 & 64.870 \\
\hline 4 & 63.051 & 63.056 & 2 & 21 & 64.900 & 64.850 \\
\hline 5 & 63.367 & 63.351 & 2 & 22 & 64.294 & 64.281 \\
\hline 5 & 63.279 & 63.416 & 2 & 22 & 64.260 & 64.266 \\
\hline 5 & 63.491 & 63.408 & 2 & 22 & 64.330 & 64.350 \\
\hline 5 & 63.498 & 63.484 & 2 & 22 & 64.349 & 64.355 \\
\hline 5 & 63.565 & 63.483 & 2 & 22 & 64.123 & 64.122 \\
\hline 5 & 63.477 & 63.498 & 2 & 22 & 64.311 & 64.372 \\
\hline 6 & 63.630 & 63.620 & 2 & 23 & 64.010 & 63.990 \\
\hline 6 & 63.670 & 63.660 & 2 & 23 & 63.880 & 63.920 \\
\hline 6 & 63.540 & 63.550 & 2 & 23 & 63.880 & 63.880 \\
\hline 6 & 63.700 & 63.730 & 2 & 23 & 64.070 & 64.070 \\
\hline 6 & 63.960 & 63.990 & 2 & 23 & 63.930 & 63.870 \\
\hline 6 & 63.970 & 63.880 & 2 & 23 & 64.000 & 63.930 \\
\hline 7 & 63.020 & 63.040 & 2 & 1 & 63.729 & 63.755 \\
\hline 7 & 63.070 & 62.970 & 2 & 1 & 63.499 & 63.554 \\
\hline 7 & 63.110 & 63.010 & 2 & 1 & 63.538 & 63.588 \\
\hline 7 & 63.120 & 62.980 & 2 & 1 & 63.633 & 63.561 \\
\hline
\end{tabular}




\begin{tabular}{|c|c|c|c|c|c|c|c|}
\hline 7 & 63.030 & 63.030 & 2 & 1 & 63.540 & 63.541 & 2 \\
\hline 7 & 63.020 & 62.950 & 2 & 1 & 63.398 & 63.619 & 2 \\
\hline 8 & 63.820 & 63.820 & 2 & 2 & 63.405 & 63.406 & 2 \\
\hline 8 & 63.850 & 63.770 & 2 & 2 & 63.320 & 63.361 & 2 \\
\hline 8 & 63.760 & 63.800 & 2 & 2 & 63.407 & 63.423 & 2 \\
\hline 8 & 63.860 & 63.920 & 2 & 2 & 63.446 & 63.460 & 2 \\
\hline 8 & 63.870 & 63.800 & 2 & 2 & 63.494 & 63.460 & 2 \\
\hline 8 & 63.810 & 63.810 & 2 & 2 & 63.663 & 63.685 & 2 \\
\hline 9 & 63.033 & 62.694 & 2 & 3 & 63.830 & 63.810 & 2 \\
\hline 9 & 62.984 & 62.694 & 2 & 3 & 63.720 & 63.730 & 2 \\
\hline 9 & 62.957 & 62.636 & 2 & 3 & 63.660 & 63.740 & 2 \\
\hline 9 & 62.738 & 62.771 & 2 & 3 & 63.760 & 63.910 & 2 \\
\hline 9 & 62.747 & 62.756 & 2 & 3 & 63.900 & 64.000 & 2 \\
\hline 9 & 62.741 & 62.803 & 2 & 3 & 63.870 & 63.840 & 2 \\
\hline 10 & 63.730 & 63.740 & 2 & 4 & 63.590 & 63.640 & 2 \\
\hline 10 & 63.670 & 63.610 & 2 & 4 & 63.580 & 63.590 & 2 \\
\hline 10 & 63.870 & 63.840 & 2 & 4 & 63.660 & 63.690 & 2 \\
\hline 10 & 63.870 & 63.980 & 2 & 4 & 63.600 & 63.640 & 2 \\
\hline 10 & 63.860 & 63.900 & 2 & 4 & 63.690 & 63.690 & 2 \\
\hline 10 & 63.830 & 63.900 & 2 & 4 & 63.760 & 63.770 & 2 \\
\hline 11 & 63.061 & 62.968 & 2 & 5 & 64.070 & 64.020 & 2 \\
\hline 11 & 63.061 & 62.968 & 2 & 5 & 64.010 & 63.910 & 2 \\
\hline 11 & 63.004 & 63.012 & 2 & 5 & 64.090 & 64.060 & 2 \\
\hline 11 & 63.020 & 63.071 & 2 & 5 & 64.080 & 64.010 & 2 \\
\hline 11 & 63.020 & 63.071 & 2 & 5 & 64.080 & 64.070 & 2 \\
\hline 11 & 63.069 & 62.934 & 2 & 5 & 63.870 & 63.940 & 2 \\
\hline 12 & 63.790 & 63.700 & 2 & 6 & 63.740 & 63.780 & 2 \\
\hline 12 & 63.750 & 63.610 & 2 & 6 & 63.750 & 63.800 & 2 \\
\hline 12 & 63.750 & 63.790 & 2 & 6 & 63.610 & 63.680 & 2 \\
\hline 12 & 63.690 & 63.770 & 2 & 6 & 63.740 & 63.820 & 2 \\
\hline 12 & 63.700 & 63.730 & 2 & 6 & 63.660 & 63.740 & 2 \\
\hline 12 & 63.630 & 63.640 & 2 & 6 & 63.750 & 63.850 & 2 \\
\hline 13 & 63.615 & 63.613 & 2 & 7 & 63.120 & 63.300 & 2 \\
\hline 13 & 63.692 & 63.664 & 2 & 7 & 63.360 & 63.490 & 2 \\
\hline 13 & 63.676 & 63.654 & 2 & 7 & 63.230 & 63.260 & 2 \\
\hline 13 & 63.586 & 63.575 & 2 & 7 & 63.340 & 63.380 & 2 \\
\hline 13 & 63.616 & 63.615 & 2 & 7 & 63.240 & 63.430 & 2 \\
\hline 13 & 63.583 & 63.601 & 2 & 7 & 63.320 & 63.400 & 2 \\
\hline 14 & 63.700 & 63.740 & 2 & 8 & 63.440 & 63.520 & 2 \\
\hline 14 & 63.670 & 63.760 & 2 & 8 & 63.450 & 63.520 & 2 \\
\hline 14 & 63.740 & 63.850 & 2 & 8 & 63.500 & 63.510 & 2 \\
\hline 14 & 63.820 & 63.930 & 2 & 8 & 63.610 & 63.590 & 2 \\
\hline 14 & 63.900 & 63.940 & 2 & 8 & 63.700 & 63.710 & 2 \\
\hline 14 & 63.810 & 63.830 & 2 & 8 & 63.670 & 63.660 & 2 \\
\hline 15 & 63.369 & 63.331 & 2 & 9 & 63.750 & 63.750 & 2 \\
\hline 15 & 63.334 & 63.333 & 2 & 9 & 63.780 & 63.690 & 2 \\
\hline 15 & 63.162 & 63.185 & 2 & 9 & 63.660 & 63.700 & 2 \\
\hline 15 & 63.473 & 63.485 & 2 & 9 & 63.690 & 63.660 & 2 \\
\hline 15 & 63.812 & 63.830 & 2 & 9 & 63.700 & 63.740 & 2 \\
\hline
\end{tabular}




\begin{tabular}{|c|c|c|c|c|c|c|}
\hline 15 & 63.292 & 63.264 & 2 & 9 & 63.840 & 63.920 \\
\hline 16 & 63.750 & 63.770 & 2 & 10 & 63.600 & 63.660 \\
\hline 16 & 63.650 & 63.700 & 2 & 10 & 63.590 & 63.500 \\
\hline 16 & 63.700 & 63.680 & 2 & 10 & 63.580 & 63.680 \\
\hline 16 & 63.890 & 63.880 & 2 & 10 & 63.650 & 63.560 \\
\hline 16 & 63.990 & 64.060 & 2 & 10 & 63.570 & 63.600 \\
\hline 16 & 63.570 & 63.490 & 2 & 10 & 63.600 & 63.650 \\
\hline 17 & 63.730 & 63.740 & 2 & 11 & 64.170 & 64.340 \\
\hline 17 & 63.800 & 63.770 & 2 & 11 & 64.260 & 64.230 \\
\hline 17 & 63.790 & 63.780 & 2 & 11 & 64.340 & 64.220 \\
\hline 17 & 63.830 & 63.830 & 2 & 11 & 64.060 & 64.160 \\
\hline 17 & 63.820 & 63.880 & 2 & 11 & 64.130 & 64.170 \\
\hline \multirow[t]{37}{*}{17} & 63.780 & 63.850 & 2 & 11 & 64.150 & 64.130 \\
\hline & & & & 12 & 64.050 & 64.140 \\
\hline & & & & 12 & 64.050 & 64.140 \\
\hline & & & & 12 & 64.230 & 64.340 \\
\hline & & & & 12 & 64.250 & 64.290 \\
\hline & & & & 12 & 64.250 & 64.290 \\
\hline & & & & 12 & 64.260 & 64.250 \\
\hline & & & & 13 & 63.390 & 63.390 \\
\hline & & & & 13 & 63.390 & 63.390 \\
\hline & & & & 13 & 63.480 & 63.590 \\
\hline & & & & 13 & 63.440 & 63.450 \\
\hline & & & & 13 & 63.440 & 63.450 \\
\hline & & & & 13 & 63.510 & 63.530 \\
\hline & & & & 14 & 63.348 & 63.392 \\
\hline & & & & 14 & 63.380 & 63.382 \\
\hline & & & & 14 & 63.185 & 63.156 \\
\hline & & & & 14 & 63.148 & 63.340 \\
\hline & & & & 14 & 63.635 & 63.639 \\
\hline & & & & 14 & 63.668 & 63.703 \\
\hline & & & & 15 & 63.730 & 63.730 \\
\hline & & & & 15 & 63.730 & 63.760 \\
\hline & & & & 15 & 63.870 & 63.820 \\
\hline & & & & 15 & 63.820 & 63.800 \\
\hline & & & & 15 & 63.760 & 63.780 \\
\hline & & & & 15 & 63.810 & 63.840 \\
\hline & & & & 16 & 63.463 & 63.471 \\
\hline & & & & 16 & 63.463 & 63.471 \\
\hline & & & & 16 & 63.536 & 63.498 \\
\hline & & & & 16 & 63.589 & 63.553 \\
\hline & & & & 16 & 63.589 & 63.553 \\
\hline & & & & 16 & 63.542 & 63.445 \\
\hline & & & & 17 & 63.440 & 63.480 \\
\hline & & & & 17 & 63.580 & 63.540 \\
\hline & & & & 17 & 63.640 & 63.610 \\
\hline & & & & 17 & 63.620 & 63.680 \\
\hline & & & & 17 & 63.690 & 63.720 \\
\hline & & & & 17 & 63.610 & 63.670 \\
\hline
\end{tabular}




$\begin{array}{llll}18 & 63.418 & 63.439 & 2 \\ 18 & 63.479 & 63.494 & 2 \\ 18 & 63.200 & 63.226 & 2 \\ 18 & 63.467 & 63.483 & 2 \\ 18 & 63.397 & 63.405 & 2 \\ 18 & 63.464 & 63.470 & 2 \\ 19 & 63.260 & 63.390 & 2 \\ 19 & 63.440 & 63.430 & 2 \\ 19 & 63.550 & 63.450 & 2 \\ 19 & 63.460 & 63.460 & 2 \\ 19 & 63.390 & 63.470 & 2 \\ 19 & 63.520 & 63.570 & 2 \\ 20 & 63.836 & 63.931 & 2 \\ 20 & 63.989 & 64.006 & 2 \\ 20 & 64.013 & 64.045 & 2 \\ 20 & 63.997 & 64.035 & 2 \\ 20 & 64.125 & 64.160 & 2 \\ 20 & 64.158 & 64.178 & 2 \\ 21 & 64.230 & 64.210 & 2 \\ 21 & 64.230 & 64.130 & 2 \\ 21 & 64.170 & 64.130 & 2 \\ 21 & 64.140 & 64.220 & 2 \\ 21 & 64.370 & 64.350 & 2 \\ 21 & 64.500 & 64.470 & 2 \\ 22 & 63.909 & 63.914 & 2 \\ 22 & 63.936 & 63.929 & 2 \\ 22 & 63.934 & 63.945 & 2 \\ 22 & 63.584 & 63.568 & 2 \\ 22 & 63.968 & 63.972 & 2 \\ 22 & 63.992 & 64.003 & 2 \\ 23 & 63.510 & 63.520 & 2 \\ 23 & 63.540 & 63.500 & 2 \\ 23 & 63.620 & 63.600 & 2 \\ 23 & 63.500 & 63.480 & 2 \\ 23 & 63.660 & 63.670 & 2 \\ 23 & 63.520 & 63.510 & 2\end{array}$


$\mathrm{MgO}$

\begin{tabular}{|c|c|c|c|c|c|c|c|}
\hline \multicolumn{4}{|c|}{ MgO: Glass Preparation } & \multicolumn{4}{|c|}{ MgO: Powder Preparation } \\
\hline Lab & Duplicate 1 & Duplicate 2 & Cement & Lab & Duplicate 1 & Duplicate 2 & Cement \\
\hline 1 & 1.8770 & 1.8770 & 1 & 1 & 1.9700 & 1.9700 & 1 \\
\hline 1 & 1.8840 & 1.8680 & 1 & 1 & 1.9700 & 1.9700 & 1 \\
\hline 1 & 1.8750 & 1.8890 & 1 & 1 & 1.9600 & 1.9200 & 1 \\
\hline 1 & 1.8970 & 1.8940 & 1 & 1 & 1.9600 & 1.9700 & 1 \\
\hline 1 & 1.8780 & 1.9140 & 1 & 1 & 1.9900 & 1.9900 & 1 \\
\hline 1 & 1.8860 & 1.9020 & 1 & 1 & 1.9900 & 2.0100 & 1 \\
\hline 2 & 1.8880 & 1.8930 & 1 & 2 & 1.8200 & 1.8300 & 1 \\
\hline 2 & 1.9120 & 1.8990 & 1 & 2 & 1.8200 & 1.8200 & 1 \\
\hline 2 & 1.8740 & 1.9140 & 1 & 2 & 1.8400 & 1.8400 & 1 \\
\hline 2 & 1.8660 & 1.8520 & 1 & 2 & 1.8200 & 1.8300 & 1 \\
\hline 2 & 1.8970 & 1.8920 & 1 & 2 & 1.8300 & 1.8300 & 1 \\
\hline 2 & 1.7920 & 1.8000 & 1 & 2 & 1.8300 & 1.8100 & 1 \\
\hline 3 & 1.8660 & 1.8580 & 1 & 3 & 1.7700 & 1.7740 & 1 \\
\hline 3 & 1.8610 & 1.8570 & 1 & 3 & 1.7860 & 1.8000 & 1 \\
\hline 3 & 1.8720 & 1.8550 & 1 & 3 & 1.8070 & 1.7990 & 1 \\
\hline 3 & 2.2730 & 2.2390 & 1 & 3 & 1.7980 & 1.8050 & 1 \\
\hline 3 & 2.2420 & 2.2570 & 1 & 3 & 1.8080 & 1.8070 & 1 \\
\hline 3 & 2.2730 & 2.2680 & 1 & 3 & 1.8020 & 1.7880 & 1 \\
\hline 4 & 1.9070 & 1.9080 & 1 & 4 & 1.7420 & 1.7460 & 1 \\
\hline 4 & 1.9040 & 1.9120 & 1 & 4 & 1.7490 & 1.7460 & 1 \\
\hline 4 & 1.9150 & 1.9060 & 1 & 4 & 1.7370 & 1.7350 & 1 \\
\hline 4 & 1.9080 & 1.9090 & 1 & 4 & 1.7300 & 1.7290 & 1 \\
\hline 4 & 1.9030 & 1.9120 & 1 & 4 & 1.7400 & 1.7410 & 1 \\
\hline 4 & 1.9030 & 1.9050 & 1 & 4 & 1.7440 & 1.7460 & 1 \\
\hline 5 & 1.9000 & 1.8900 & 1 & 5 & 1.9000 & 1.9300 & 1 \\
\hline 5 & 1.9000 & 1.8900 & 1 & 5 & 1.8900 & 1.9100 & 1 \\
\hline 5 & 1.9000 & 1.8900 & 1 & 5 & 1.9100 & 1.9100 & 1 \\
\hline 5 & 1.9000 & 1.9000 & 1 & 5 & 1.9300 & 1.9200 & 1 \\
\hline 5 & 1.9000 & 1.8800 & 1 & 5 & 1.8900 & 1.8900 & 1 \\
\hline 5 & 1.8900 & 1.9000 & 1 & 5 & 1.9300 & 1.9200 & 1 \\
\hline 6 & 1.8980 & 1.8970 & 1 & 6 & 1.8500 & 1.8500 & 1 \\
\hline 6 & 1.8940 & 1.8990 & 1 & 6 & 1.8400 & 1.8400 & 1 \\
\hline 6 & 1.8990 & 1.8980 & 1 & 6 & 1.8400 & 1.8400 & 1 \\
\hline 6 & 1.9170 & 1.9110 & 1 & 6 & 1.8700 & 1.8500 & 1 \\
\hline 6 & 1.9080 & 1.9030 & 1 & 6 & 1.8300 & 1.8400 & 1 \\
\hline 6 & 1.9060 & 1.9140 & 1 & 6 & 1.8400 & 1.8400 & 1 \\
\hline 7 & 1.9100 & 1.9300 & 1 & 7 & 1.8600 & 1.8700 & 1 \\
\hline 7 & 1.9000 & 1.9100 & 1 & 7 & 1.8300 & 1.8400 & 1 \\
\hline 7 & 1.9200 & 1.9200 & 1 & 7 & 1.8300 & 1.8300 & 1 \\
\hline 7 & 1.9000 & 1.9100 & 1 & 7 & 1.8500 & 1.8600 & 1 \\
\hline 7 & 1.9000 & 1.9000 & 1 & 7 & 1.8300 & 1.8300 & 1 \\
\hline 7 & 1.9000 & 1.9000 & 1 & 7 & 1.8300 & 1.8200 & 1 \\
\hline 8 & 1.9800 & 1.9900 & 1 & 8 & 1.9400 & 1.9400 & 1 \\
\hline 8 & 2.0000 & 2.0100 & 1 & 8 & 1.9300 & 1.9300 & 1 \\
\hline 8 & 1.9800 & 1.9900 & 1 & 8 & 1.9300 & 1.9200 & 1 \\
\hline 8 & 1.9800 & 1.9700 & 1 & 8 & 1.9300 & 1.9400 & 1 \\
\hline
\end{tabular}




\begin{tabular}{|c|c|c|c|c|c|c|}
\hline 8 & 1.9800 & 2.0000 & 1 & 8 & 1.9300 & 1.9300 \\
\hline 8 & 2.0000 & 1.9800 & 1 & 8 & 1.9300 & 1.9200 \\
\hline 9 & 1.9280 & 1.9350 & 1 & 9 & 1.8400 & 1.8400 \\
\hline 9 & 1.9380 & 1.9250 & 1 & 9 & 1.8500 & 1.8500 \\
\hline 9 & 1.9340 & 1.9280 & 1 & 9 & 1.8400 & 1.8400 \\
\hline 9 & 1.9370 & 1.9300 & 1 & 9 & 1.8600 & 1.8600 \\
\hline 9 & 1.9280 & 1.9240 & 1 & 9 & 1.8400 & 1.8500 \\
\hline 9 & 1.9270 & 1.9370 & 1 & 9 & 1.8400 & 1.8400 \\
\hline 10 & 1.8900 & 1.8600 & 1 & 10 & 1.9800 & 1.9800 \\
\hline 10 & 1.8800 & 1.8600 & 1 & 10 & 2.0000 & 2.0000 \\
\hline 10 & 1.8700 & 1.8900 & 1 & 10 & 2.0000 & 2.0100 \\
\hline 10 & 1.8600 & 1.9000 & 1 & 10 & 1.9700 & 1.9700 \\
\hline 10 & 1.8500 & 1.8700 & 1 & 10 & 2.0000 & 2.0000 \\
\hline 10 & 1.8500 & 1.8800 & 1 & 10 & 2.0100 & 2.0000 \\
\hline 11 & 1.8900 & 1.8800 & 1 & 11 & 1.9700 & 1.9700 \\
\hline 11 & 1.9000 & 1.8900 & 1 & 11 & 1.8800 & 1.8800 \\
\hline 11 & 1.8800 & 1.8800 & 1 & 11 & 1.8700 & 1.8700 \\
\hline 11 & 1.8700 & 1.8700 & 1 & 11 & 1.9000 & 1.8900 \\
\hline 11 & 1.8800 & 1.8800 & 1 & 11 & 1.8700 & 1.8700 \\
\hline 11 & 1.8800 & 1.8800 & 1 & 11 & 1.8600 & 1.8800 \\
\hline 12 & 1.9000 & 1.9300 & 1 & 12 & 1.9200 & 1.9200 \\
\hline 12 & 1.9300 & 1.9200 & 1 & 12 & 1.9400 & 1.9300 \\
\hline 12 & 1.9100 & 1.9000 & 1 & 12 & 1.9200 & 1.9200 \\
\hline 12 & 1.9400 & 1.9300 & 1 & 12 & 1.9000 & 1.9200 \\
\hline 12 & 1.9400 & 1.9100 & 1 & 12 & 1.9200 & 1.9300 \\
\hline 12 & 1.9100 & 1.9100 & 1 & 12 & 1.9100 & 1.9200 \\
\hline 13 & 1.9300 & 1.9300 & 1 & 13 & 1.9123 & 1.9123 \\
\hline 13 & 1.9500 & 1.9300 & 1 & 13 & 1.9128 & 1.9106 \\
\hline 13 & 1.9300 & 1.9400 & 1 & 13 & 1.9157 & 1.9200 \\
\hline 13 & 1.9400 & 1.9400 & 1 & 13 & 1.9094 & 1.9113 \\
\hline 13 & 1.9200 & 1.9300 & 1 & 13 & 1.9057 & 1.9098 \\
\hline 13 & 1.9200 & 1.9400 & 1 & 13 & 1.9168 & 1.9195 \\
\hline 14 & 1.9110 & 1.9340 & 1 & 14 & 1.7890 & 1.8010 \\
\hline 14 & 1.8870 & 1.9270 & 1 & 14 & 1.8160 & 1.7740 \\
\hline 14 & 1.8790 & 1.9350 & 1 & 14 & 1.8100 & 1.7910 \\
\hline 14 & 1.8740 & 1.8800 & 1 & 14 & 1.8110 & 1.8110 \\
\hline 14 & 1.9110 & 1.9030 & 1 & 14 & 1.8170 & 1.8230 \\
\hline 14 & 1.9100 & 1.8990 & 1 & 14 & 1.7940 & 1.7940 \\
\hline 15 & 2.0000 & 2.0100 & 1 & 15 & 2.0100 & 2.0200 \\
\hline 15 & 2.0000 & 2.0000 & 1 & 15 & 1.8700 & 1.8700 \\
\hline 15 & 2.0000 & 2.0100 & 1 & 15 & 1.9000 & 1.9000 \\
\hline 15 & 2.0000 & 2.0200 & 1 & 15 & 1.8500 & 1.8700 \\
\hline 15 & 2.0200 & 2.0000 & 1 & 15 & 1.8400 & 1.8700 \\
\hline 15 & 2.0200 & 2.0000 & 1 & 15 & 1.8700 & 1.8700 \\
\hline 16 & 1.9000 & 1.9100 & 1 & 16 & 1.8140 & 1.8170 \\
\hline 16 & 1.9000 & 1.9200 & 1 & 16 & 1.8120 & 1.8170 \\
\hline 16 & 1.9100 & 1.8900 & 1 & 16 & 1.8170 & 1.8220 \\
\hline 16 & 1.8900 & 1.8800 & 1 & 16 & 1.0824 & 1.8280 \\
\hline 16 & 1.9000 & 1.9100 & 1 & 16 & 1.8280 & 1.8310 \\
\hline
\end{tabular}




\begin{tabular}{|c|c|c|c|c|c|c|}
\hline 16 & 1.8900 & 1.8900 & 1 & 16 & 1.8230 & 1.8160 \\
\hline 17 & 1.9100 & 1.9000 & 1 & 17 & 1.8150 & 1.8080 \\
\hline 17 & 1.8900 & 1.9100 & 1 & 17 & 1.8350 & 1.8360 \\
\hline 17 & 1.9100 & 1.8900 & 1 & 17 & 1.8150 & 1.8240 \\
\hline 17 & 1.8900 & 1.8900 & 1 & 17 & 1.8160 & 1.8130 \\
\hline 17 & 1.8800 & 1.8900 & 1 & 17 & 1.8040 & 1.8050 \\
\hline 17 & 1.8800 & 1.9000 & 1 & 17 & 1.8130 & 1.8120 \\
\hline 18 & 1.9096 & 1.9079 & 1 & 18 & 1.7600 & 1.7400 \\
\hline 18 & 1.9196 & 1.9159 & 1 & 18 & 1.7800 & 1.7700 \\
\hline 18 & 1.9109 & 1.9035 & 1 & 18 & 1.8000 & 1.8000 \\
\hline 18 & 1.9135 & 1.9040 & 1 & 18 & 1.7400 & 1.7600 \\
\hline 18 & 1.9179 & 1.9043 & 1 & 18 & 1.7800 & 1.7600 \\
\hline 18 & 1.9092 & 1.9098 & 1 & 18 & 1.7800 & 1.7700 \\
\hline 19 & 1.9000 & 1.9000 & 1 & 19 & 1.8400 & 1.8400 \\
\hline 19 & 1.9100 & 1.9000 & 1 & 19 & 1.8300 & 1.8300 \\
\hline 19 & 1.9100 & 1.8900 & 1 & 19 & 1.8300 & 1.8300 \\
\hline 19 & 1.8900 & 1.9000 & 1 & 19 & 1.8300 & 1.8400 \\
\hline 19 & 1.8900 & 1.8800 & 1 & 19 & 1.8300 & 1.8300 \\
\hline 19 & 1.9000 & 1.8900 & 1 & 19 & 1.8300 & 1.8300 \\
\hline 1 & 1.0500 & 1.0500 & 2 & 20 & 1.9100 & 1.9200 \\
\hline 1 & 1.0390 & 1.0410 & 2 & 20 & 1.8800 & 1.8900 \\
\hline 1 & 1.0470 & 1.0420 & 2 & 20 & 1.9200 & 1.9000 \\
\hline 1 & 1.0510 & 1.0430 & 2 & 20 & 1.9100 & 1.9100 \\
\hline 1 & 1.0530 & 1.0500 & 2 & 20 & 1.9000 & 1.8900 \\
\hline 1 & 1.0540 & 1.0450 & 2 & 20 & 1.9100 & 1.9000 \\
\hline 2 & 1.0480 & 1.0710 & 2 & 21 & 1.8082 & 1.8069 \\
\hline 2 & 1.0540 & 1.0420 & 2 & 21 & 1.8221 & 1.8186 \\
\hline 2 & 1.0380 & 1.0620 & 2 & 21 & 1.8259 & 1.8208 \\
\hline 2 & 0.9970 & 1.0050 & 2 & 21 & 1.8047 & 1.8008 \\
\hline 2 & 1.0310 & 1.0380 & 2 & 21 & 1.8107 & 1.8168 \\
\hline 2 & 1.0550 & 1.0430 & 2 & 21 & 1.8250 & 1.8228 \\
\hline 3 & 1.0280 & 1.0600 & 2 & 22 & 1.6300 & 1.6390 \\
\hline 3 & 1.0150 & 1.0240 & 2 & 22 & 1.6220 & 1.6160 \\
\hline 3 & 1.0450 & 1.0060 & 2 & 22 & 1.6440 & 1.6480 \\
\hline 3 & 1.4310 & 1.4380 & 2 & 22 & 1.6220 & 1.6270 \\
\hline 3 & 1.4350 & 1.4480 & 2 & 22 & 1.6290 & 1.6270 \\
\hline 3 & 1.4260 & 1.4410 & 2 & 22 & 1.4950 & 1.4870 \\
\hline 4 & 1.0560 & 1.0520 & 2 & 23 & 1.8200 & 1.8100 \\
\hline 4 & 1.0580 & 1.0620 & 2 & 23 & 1.8200 & 1.8300 \\
\hline 4 & 1.0600 & 1.0570 & 2 & 23 & 1.8000 & 1.8100 \\
\hline 4 & 1.0640 & 1.0670 & 2 & 23 & 1.8300 & 1.8300 \\
\hline 4 & 1.0560 & 1.0600 & 2 & 23 & 1.8200 & 1.8200 \\
\hline 4 & 1.0680 & 1.0610 & 2 & 23 & 1.8200 & 1.8400 \\
\hline 5 & 1.0800 & 1.0700 & 2 & 24 & 1.8400 & 1.8400 \\
\hline 5 & 1.0700 & 1.0800 & 2 & 24 & 1.8400 & 1.8400 \\
\hline 5 & 1.0700 & 1.0700 & 2 & 24 & 1.8200 & 1.8200 \\
\hline 5 & 1.0700 & 1.0700 & 2 & 24 & 1.8300 & 1.8300 \\
\hline 5 & 1.0600 & 1.0500 & 2 & 24 & 1.8300 & 1.8300 \\
\hline 5 & 1.0600 & 1.0600 & 2 & 24 & 1.8300 & 1.8300 \\
\hline
\end{tabular}




\begin{tabular}{|c|c|c|c|c|c|c|}
\hline 6 & 1.0650 & 1.0630 & 2 & 25 & 1.8321 & 1.8231 \\
\hline 6 & 1.0630 & 1.0680 & 2 & 25 & 1.8293 & 1.8193 \\
\hline 6 & 1.0660 & 1.0670 & 2 & 25 & 1.8236 & 1.8186 \\
\hline 6 & 1.0730 & 1.0620 & 2 & 25 & 1.8116 & 1.8084 \\
\hline 6 & 1.0650 & 1.0660 & 2 & 25 & 1.8206 & 1.8212 \\
\hline 6 & 1.0650 & 1.0630 & 2 & 25 & 1.8239 & 1.8197 \\
\hline 7 & 1.0700 & 1.0700 & 2 & 26 & 1.8200 & 1.8200 \\
\hline 7 & 1.0600 & 1.0700 & 2 & 26 & 1.8200 & 1.8200 \\
\hline 7 & 1.0700 & 1.0700 & 2 & 26 & 1.8000 & 1.8300 \\
\hline 7 & 1.0700 & 1.0600 & 2 & 26 & 1.8200 & 1.8300 \\
\hline 7 & 1.0600 & 1.0700 & 2 & 26 & 1.8400 & 1.8200 \\
\hline 7 & 1.0600 & 1.0600 & 2 & 26 & 1.8200 & 1.8200 \\
\hline 8 & 1.1100 & 1.0900 & 2 & 1 & 1.1600 & 1.1400 \\
\hline 8 & 1.0900 & 1.1000 & 2 & 1 & 1.1500 & 1.1300 \\
\hline 8 & 1.1100 & 1.1000 & 2 & 1 & 1.1200 & 1.1200 \\
\hline 8 & 1.1000 & 1.0900 & 2 & 1 & 1.1600 & 1.1600 \\
\hline 8 & 1.1200 & 1.1000 & 2 & 1 & 1.1500 & 1.1300 \\
\hline 8 & 1.1000 & 1.1000 & 2 & 1 & 1.1500 & 1.1200 \\
\hline 9 & 1.0710 & 1.0740 & 2 & 2 & 1.1400 & 1.1400 \\
\hline 9 & 1.0760 & 1.0670 & 2 & 2 & 1.1200 & 1.1200 \\
\hline 9 & 1.0810 & 1.0660 & 2 & 2 & 1.1200 & 1.1400 \\
\hline 9 & 1.0750 & 1.0810 & 2 & 2 & 1.1200 & 1.1300 \\
\hline 9 & 1.0850 & 1.0810 & 2 & 2 & 1.1100 & 1.1400 \\
\hline 9 & 1.0830 & 1.0740 & 2 & 2 & 1.1100 & 1.1200 \\
\hline 10 & 1.1100 & 1.0800 & 2 & 3 & 1.1360 & 1.1450 \\
\hline 10 & 1.1200 & 1.1000 & 2 & 3 & 1.1460 & 1.1500 \\
\hline 10 & 1.1000 & 1.1000 & 2 & 3 & 1.1320 & 1.1350 \\
\hline 10 & 1.1000 & 1.0800 & 2 & 3 & 1.1610 & 1.1520 \\
\hline 10 & 1.0900 & 1.1000 & 2 & 3 & 1.1430 & 1.1370 \\
\hline 10 & 1.1100 & 1.1000 & 2 & 3 & 1.1360 & 1.1410 \\
\hline 11 & 1.0500 & 1.0500 & 2 & 4 & 1.0140 & 1.0140 \\
\hline 11 & 1.0500 & 1.0500 & 2 & 4 & 1.0480 & 1.0480 \\
\hline 11 & 1.0500 & 1.0500 & 2 & 4 & 0.9970 & 0.9990 \\
\hline 11 & 1.0400 & 1.0500 & 2 & 4 & 1.0120 & 1.0090 \\
\hline 11 & 1.0500 & 1.0400 & 2 & 4 & 0.9960 & 0.9990 \\
\hline 11 & 1.0500 & 1.0500 & 2 & 4 & 0.9940 & 0.9930 \\
\hline 12 & 1.1000 & 1.0900 & 2 & 5 & 1.1000 & 1.0900 \\
\hline 12 & 1.1200 & 1.0900 & 2 & 5 & 1.0800 & 1.0500 \\
\hline 12 & 1.1000 & 1.0700 & 2 & 5 & 1.0700 & 1.0600 \\
\hline 12 & 1.1100 & 1.0800 & 2 & 5 & 1.0700 & 1.0700 \\
\hline 12 & 1.0900 & 1.0900 & 2 & 5 & 1.0900 & 1.1000 \\
\hline 12 & 1.0900 & 1.0900 & 2 & 5 & 1.0900 & 1.0500 \\
\hline 13 & 1.0800 & 1.0800 & 2 & 6 & 1.1000 & 1.0900 \\
\hline 13 & 1.0600 & 1.0700 & 2 & 6 & 1.0900 & 1.0900 \\
\hline 13 & 1.0600 & 1.0700 & 2 & 6 & 1.0800 & 1.0900 \\
\hline 13 & 1.0500 & 1.0700 & 2 & 6 & 1.1000 & 1.1000 \\
\hline 13 & 1.0500 & 1.0600 & 2 & 6 & 1.0900 & 1.0900 \\
\hline 13 & 1.0500 & 1.0600 & 2 & 6 & 1.0800 & 1.0800 \\
\hline 14 & 1.0330 & 1.0650 & 2 & 7 & 1.0800 & 1.0700 \\
\hline
\end{tabular}




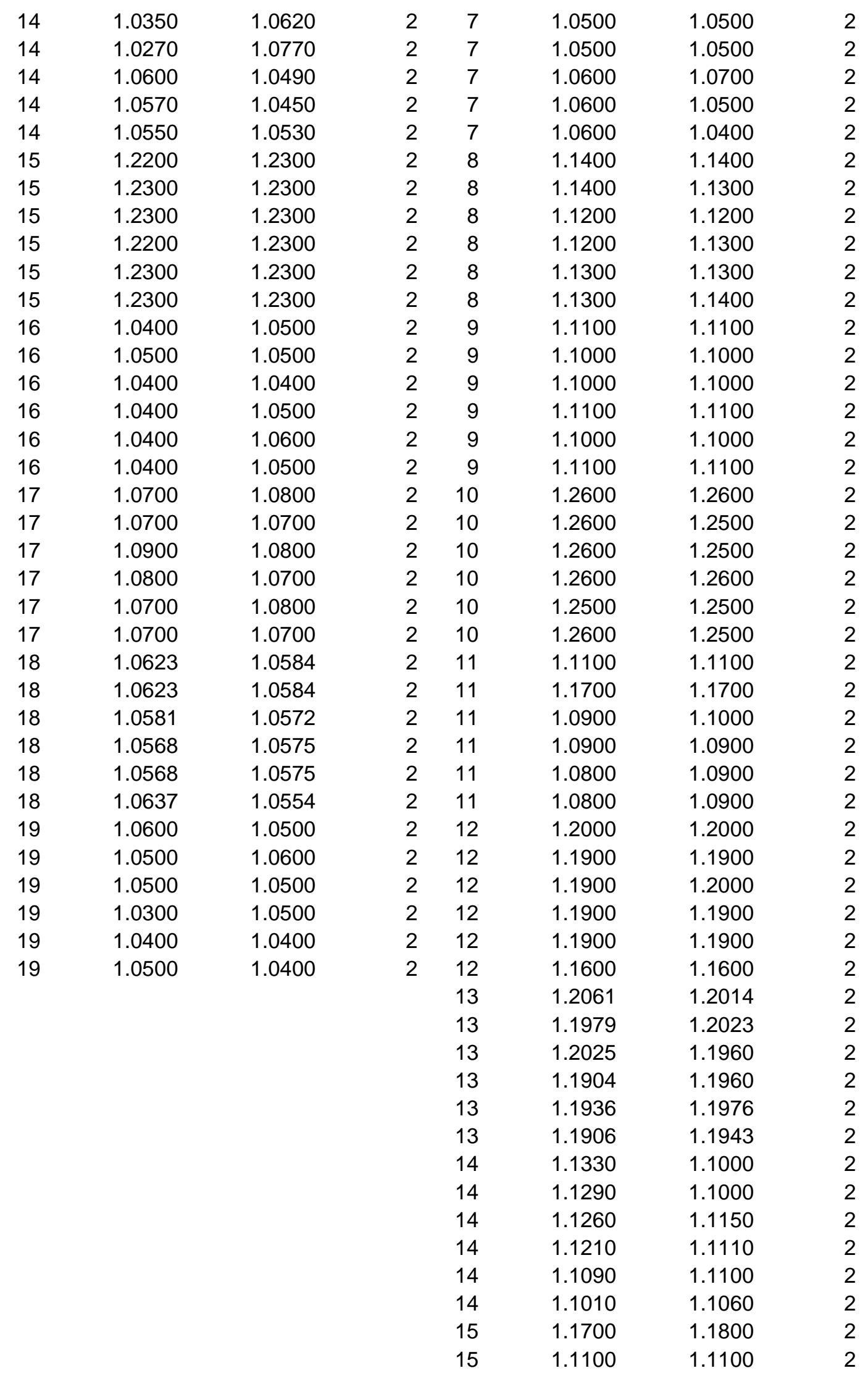




$\begin{array}{llll}15 & 1.1200 & 1.1100 & 2 \\ 15 & 1.1800 & 1.1800 & 2 \\ 15 & 1.1400 & 1.1500 & 2 \\ 15 & 1.1100 & 1.1200 & 2 \\ 16 & 1.0660 & 1.0670 & 2 \\ 16 & 1.0460 & 1.0560 & 2 \\ 16 & 1.0500 & 1.0460 & 2 \\ 16 & 1.0690 & 1.0670 & 2 \\ 16 & 1.0600 & 1.0580 & 2 \\ 16 & 1.0570 & 1.0600 & 2 \\ 17 & 1.0750 & 1.0740 & 2 \\ 17 & 1.0660 & 1.0680 & 2 \\ 17 & 1.0670 & 1.0690 & 2 \\ 17 & 1.0770 & 1.0740 & 2 \\ 17 & 1.0760 & 1.0770 & 2 \\ 17 & 1.0690 & 1.0730 & 2 \\ 18 & 1.0800 & 1.0600 & 2 \\ 18 & 1.0300 & 1.0300 & 2 \\ 18 & 1.0400 & 1.0300 & 2 \\ 18 & 1.0600 & 1.0400 & 2 \\ 18 & 1.0500 & 1.0300 & 2 \\ 18 & 1.0400 & 1.0300 & 2 \\ 19 & 1.0700 & 1.0700 & 2 \\ 19 & 1.0600 & 1.0600 & 2 \\ 19 & 1.0600 & 1.0600 & 2 \\ 19 & 1.0600 & 1.0700 & 2 \\ 19 & 1.0500 & 1.0600 & 2 \\ 19 & 1.0500 & 1.0500 & 2 \\ 20 & 1.1500 & 1.1600 & 2 \\ 20 & 1.1300 & 1.1300 & 2 \\ 20 & 1.1500 & 1.1500 & 2 \\ 20 & 1.1400 & 1.1300 & 2 \\ 20 & 1.1400 & 1.1400 & 2 \\ 20 & 1.1200 & 1.1200 & 2 \\ 21 & 1.0574 & 1.0501 & 2 \\ 21 & 1.0398 & 1.0397 & 2 \\ 21 & 1.0372 & 1.0378 & 2 \\ 21 & 1.0557 & 1.0565 & 2 \\ 21 & 1.0555 & 1.0554 & 2 \\ 21 & 1.0566 & 1.0563 & 2 \\ 22 & 0.9490 & 0.9470 & 2 \\ 22 & 0.9520 & 0.9520 & 2 \\ 22 & 0.9370 & 0.9340 & 2 \\ 22 & 0.9370 & 0.9390 & 2 \\ 22 & 0.9340 & 0.9300 & 2 \\ 23 & 0.9300 & 0.9290 & 2 \\ 12 & 1.0400 & 1.0500 & 2 \\ & 1.0500 & 1.0400 & 2 \\ 12 & 1.0300 & 1.0500 & 2 \\ 12 & & & \\ 12 & \end{array}$




$\begin{array}{llll}23 & 1.0500 & 1.0400 & 2 \\ 23 & 1.0400 & 1.0500 & 2 \\ 23 & 1.0400 & 1.0500 & 2 \\ 24 & 1.0800 & 1.0800 & 2 \\ 24 & 1.0800 & 1.0800 & 2 \\ 24 & 1.0800 & 1.0900 & 2 \\ 24 & 1.0900 & 1.0900 & 2 \\ 24 & 1.0900 & 1.1000 & 2 \\ 24 & 1.0800 & 1.0800 & 2 \\ 25 & 1.0235 & 1.0222 & 2 \\ 25 & 1.0235 & 1.0222 & 2 \\ 25 & 1.0277 & 1.0299 & 2 \\ 25 & 1.0251 & 1.0251 & 2 \\ 25 & 1.0251 & 1.0251 & 2 \\ 25 & 1.0221 & 1.0230 & 2 \\ 26 & 1.0500 & 1.0600 & 2 \\ 26 & 1.0600 & 1.0500 & 2 \\ 26 & 1.0400 & 1.0500 & 2 \\ 26 & 1.0500 & 1.0500 & 2 \\ 26 & 1.0400 & 1.0600 & 2 \\ 26 & 1.0500 & 1.0400 & 2\end{array}$




\begin{tabular}{|c|c|c|c|c|c|c|c|}
\hline \multicolumn{4}{|c|}{ SO3: Glass Preparation } & \multicolumn{4}{|c|}{ SO3: Powder Preparation } \\
\hline Lab & Duplicate 1 & Duplicate 2 & Cement & Lab & Duplicate 1 & Duplicate 2 & Cement \\
\hline 1 & 2.8600 & 2.8700 & 1 & 1 & 2.7900 & 2.7900 & 1 \\
\hline 1 & 2.9000 & 2.9000 & 1 & 1 & 2.8200 & 2.8300 & 1 \\
\hline 1 & 2.9100 & 2.8900 & 1 & 1 & 2.8300 & 2.8400 & 1 \\
\hline 1 & 2.8600 & 2.8600 & 1 & 1 & 2.8100 & 2.8100 & 1 \\
\hline 1 & 2.8800 & 2.8700 & 1 & 1 & 2.8300 & 2.8200 & 1 \\
\hline 1 & 2.8600 & 2.8700 & 1 & 1 & 2.8400 & 2.8400 & 1 \\
\hline 2 & 2.8700 & 2.8600 & 1 & 2 & 2.7300 & 2.7500 & 1 \\
\hline 2 & 2.8800 & 2.8800 & 1 & 2 & 2.7300 & 2.7500 & 1 \\
\hline 2 & 2.8300 & 2.8400 & 1 & 2 & 2.7500 & 2.7500 & 1 \\
\hline 2 & 2.8300 & 2.8300 & 1 & 2 & 2.7400 & 2.7500 & 1 \\
\hline 2 & 2.8500 & 2.8500 & 1 & 2 & 2.7200 & 2.7300 & 1 \\
\hline 2 & 2.8300 & 2.8400 & 1 & 2 & 2.7300 & 2.7500 & 1 \\
\hline 3 & 2.7900 & 2.8000 & 1 & 3 & 2.8800 & 2.8800 & 1 \\
\hline 3 & 2.8000 & 2.8000 & 1 & 3 & 2.8800 & 2.8900 & 1 \\
\hline 3 & 2.7900 & 2.8000 & 1 & 3 & 2.9000 & 2.9100 & 1 \\
\hline 3 & 2.7400 & 2.7200 & 1 & 3 & 2.8900 & 2.9000 & 1 \\
\hline 3 & 2.7400 & 2.7300 & 1 & 3 & 2.9000 & 2.9000 & 1 \\
\hline 3 & 2.7300 & 2.7300 & 1 & 3 & 2.9000 & 2.9100 & 1 \\
\hline 4 & 2.8260 & 2.8200 & 1 & 4 & 2.9000 & 2.9100 & 1 \\
\hline 4 & 2.7900 & 2.7960 & 1 & 4 & 2.9000 & 2.9000 & 1 \\
\hline 4 & 2.8670 & 2.8620 & 1 & 4 & 2.8700 & 2.8800 & 1 \\
\hline 4 & 2.8290 & 2.8270 & 1 & 4 & 2.9100 & 2.9100 & 1 \\
\hline 4 & 2.8290 & 2.8250 & 1 & 4 & 2.9100 & 2.9200 & 1 \\
\hline 4 & 2.8300 & 2.8270 & 1 & 4 & 2.9100 & 2.9200 & 1 \\
\hline 5 & 2.8910 & 2.8770 & 1 & 5 & 2.9090 & 2.9080 & 1 \\
\hline 5 & 2.8930 & 2.8840 & 1 & 5 & 2.9020 & 2.9030 & 1 \\
\hline 5 & 2.8900 & 2.8890 & 1 & 5 & 2.9880 & 2.9880 & 1 \\
\hline 5 & 2.8320 & 2.8630 & 1 & 5 & 2.9340 & 2.9350 & 1 \\
\hline 5 & 2.8410 & 2.8580 & 1 & 5 & 2.8920 & 2.8940 & 1 \\
\hline 5 & 2.7280 & 2.7290 & 1 & 5 & 2.9310 & 2.9320 & 1 \\
\hline 6 & 2.8300 & 2.8140 & 1 & 6 & 2.9300 & 2.9300 & 1 \\
\hline 6 & 2.8320 & 2.8510 & 1 & 6 & 2.9300 & 2.9300 & 1 \\
\hline 6 & 2.8220 & 2.8680 & 1 & 6 & 2.9200 & 2.9200 & 1 \\
\hline 6 & 2.8690 & 2.8680 & 1 & 6 & 2.9200 & 2.9300 & 1 \\
\hline 6 & 2.8240 & 2.8250 & 1 & 6 & 2.9400 & 2.9300 & 1 \\
\hline 6 & 2.8520 & 2.8240 & 1 & 6 & 2.9200 & 2.9200 & 1 \\
\hline 7 & 2.9110 & 2.9100 & 1 & 7 & 2.8770 & 2.8770 & 1 \\
\hline 7 & 2.8810 & 2.8820 & 1 & 7 & 2.8940 & 2.8980 & 1 \\
\hline 7 & 2.8870 & 2.8890 & 1 & 7 & 2.9970 & 3.0030 & 1 \\
\hline 7 & 2.9170 & 2.9120 & 1 & 7 & 2.9580 & 2.9410 & 1 \\
\hline 7 & 2.8980 & 2.9010 & 1 & 7 & 2.9570 & 2.9700 & 1 \\
\hline 7 & 2.9070 & 2.9080 & 1 & 7 & 2.9760 & 2.9770 & 1 \\
\hline 8 & 2.8800 & 2.8800 & 1 & 8 & 2.9800 & 2.9800 & 1 \\
\hline 8 & 2.8700 & 2.8800 & 1 & 8 & 2.9900 & 2.9900 & 1 \\
\hline 8 & 2.8700 & 2.8800 & 1 & 8 & 2.9800 & 2.9800 & 1 \\
\hline 8 & 2.8700 & 2.8600 & 1 & 8 & 2.9700 & 2.9800 & 1 \\
\hline
\end{tabular}




\begin{tabular}{|c|c|c|c|c|c|c|}
\hline 8 & 2.8900 & 2.9000 & 1 & 8 & 2.9500 & 2.9500 \\
\hline 8 & 2.8600 & 2.8700 & 1 & 8 & 2.9900 & 3.0000 \\
\hline 9 & 2.8160 & 2.7940 & 1 & 9 & 2.7597 & 2.7489 \\
\hline 9 & 2.7880 & 2.7980 & 1 & 9 & 2.7569 & 2.7507 \\
\hline 9 & 2.8370 & 2.7970 & 1 & 9 & 2.7548 & 2.7480 \\
\hline 9 & 2.7680 & 2.7880 & 1 & 9 & 2.7475 & 2.7449 \\
\hline 9 & 2.8110 & 2.8220 & 1 & 9 & 2.7665 & 2.7632 \\
\hline 9 & 2.8030 & 2.8010 & 1 & 9 & 2.7494 & 2.7444 \\
\hline 10 & 2.7900 & 2.7910 & 1 & 10 & 2.9300 & 2.9400 \\
\hline 10 & 2.7580 & 2.7610 & 1 & 10 & 2.9500 & 2.9500 \\
\hline 10 & 2.7600 & 2.7670 & 1 & 10 & 2.9600 & 2.9400 \\
\hline 10 & 2.7820 & 2.7860 & 1 & 10 & 2.9400 & 2.9300 \\
\hline 10 & 2.8060 & 2.8020 & 1 & 10 & 2.9200 & 2.9300 \\
\hline 10 & 2.7930 & 2.7900 & 1 & 10 & 2.9100 & 2.9200 \\
\hline 11 & 2.9480 & 2.9470 & 1 & 11 & 2.9570 & 2.9590 \\
\hline 11 & 2.9380 & 2.9360 & 1 & 11 & 2.9620 & 2.9740 \\
\hline 11 & 2.9420 & 2.9370 & 1 & 11 & 2.9820 & 2.9470 \\
\hline 11 & 2.9430 & 2.9400 & 1 & 11 & 2.9870 & 2.9820 \\
\hline 11 & 2.9460 & 2.9440 & 1 & 11 & 2.9880 & 2.9840 \\
\hline 11 & 2.9430 & 2.9460 & 1 & 11 & 3.0360 & 3.0230 \\
\hline 12 & 2.8600 & 2.9000 & 1 & 12 & 2.9110 & 2.9120 \\
\hline 12 & 2.9000 & 2.8800 & 1 & 12 & 2.9030 & 2.9080 \\
\hline 12 & 2.9300 & 2.9100 & 1 & 12 & 2.9100 & 2.9070 \\
\hline 12 & 2.8700 & 2.8700 & 1 & 12 & 2.8900 & 2.8970 \\
\hline 12 & 2.8900 & 2.8800 & 1 & 12 & 2.9130 & 2.9140 \\
\hline 12 & 2.8800 & 2.8800 & 1 & 12 & 2.9120 & 2.9100 \\
\hline 13 & 2.8700 & 2.8800 & 1 & 13 & 2.9200 & 2.9300 \\
\hline 13 & 2.8600 & 2.8600 & 1 & 13 & 2.9000 & 2.9000 \\
\hline 13 & 2.8600 & 2.8400 & 1 & 13 & 2.9200 & 2.9200 \\
\hline 13 & 2.8500 & 2.8500 & 1 & 13 & 2.8900 & 2.9000 \\
\hline 13 & 2.8800 & 2.8700 & 1 & 13 & 2.9100 & 2.9200 \\
\hline 13 & 2.8800 & 2.8800 & 1 & 13 & 2.9600 & 2.9600 \\
\hline 14 & 2.9200 & 2.9200 & 1 & 14 & 2.9200 & 2.9160 \\
\hline 14 & 2.9100 & 2.9200 & 1 & 14 & 2.8850 & 2.8820 \\
\hline 14 & 2.9200 & 2.9200 & 1 & 14 & 2.9850 & 2.9860 \\
\hline 14 & 2.9100 & 2.9100 & 1 & 14 & 2.9690 & 2.9700 \\
\hline 14 & 2.9100 & 2.9100 & 1 & 14 & 2.9790 & 2.9820 \\
\hline 14 & 2.9100 & 2.9100 & 1 & 14 & 2.9680 & 2.9680 \\
\hline 15 & 2.8200 & 2.8300 & 1 & 15 & 2.9000 & 2.8800 \\
\hline 15 & 2.8200 & 2.8200 & 1 & 15 & 2.9200 & 2.9300 \\
\hline 15 & 2.8200 & 2.8200 & 1 & 15 & 2.9300 & 2.9200 \\
\hline 15 & 2.8200 & 2.8100 & 1 & 15 & 2.8800 & 2.8600 \\
\hline 15 & 2.8000 & 2.8100 & 1 & 15 & 2.9100 & 2.9200 \\
\hline 15 & 2.8100 & 2.8000 & 1 & 15 & 2.9100 & 2.9300 \\
\hline 16 & 2.8200 & 2.8300 & 1 & 16 & 2.9000 & 2.9000 \\
\hline 16 & 2.8300 & 2.8300 & 1 & 16 & 2.9100 & 2.9100 \\
\hline 16 & 2.8400 & 2.8500 & 1 & 16 & 2.8900 & 2.9000 \\
\hline 16 & 2.8400 & 2.8400 & 1 & 16 & 2.8800 & 2.8900 \\
\hline 16 & 2.8200 & 2.8200 & 1 & 16 & 2.9000 & 2.9100 \\
\hline
\end{tabular}




\begin{tabular}{|c|c|c|c|c|c|c|c|}
\hline 16 & 2.8400 & 2.8300 & 1 & 16 & 2.9100 & 2.9000 & 1 \\
\hline 1 & 3.6100 & 3.6100 & 2 & 17 & 2.8900 & 2.9000 & 1 \\
\hline 1 & 3.6400 & 3.6700 & 2 & 17 & 2.9100 & 2.9200 & 1 \\
\hline 1 & 3.6100 & 3.6600 & 2 & 17 & 2.9200 & 2.9200 & 1 \\
\hline 1 & 3.6300 & 3.6300 & 2 & 17 & 2.9200 & 2.9200 & 1 \\
\hline 1 & 3.6100 & 3.5800 & 2 & 17 & 2.9100 & 2.9100 & 1 \\
\hline 1 & 3.6300 & 3.6500 & 2 & 17 & 2.9200 & 2.9200 & 1 \\
\hline 2 & 3.5900 & 3.6000 & 2 & 18 & 2.8789 & 2.8814 & 1 \\
\hline 2 & 3.5800 & 3.5700 & 2 & 18 & 2.9255 & 2.9284 & 1 \\
\hline 2 & 3.6100 & 3.6300 & 2 & 18 & 2.9338 & 2.9351 & 1 \\
\hline 2 & 3.5400 & 3.5500 & 2 & 18 & 2.8918 & 2.8878 & 1 \\
\hline 2 & 3.6000 & 3.6100 & 2 & 18 & 2.9271 & 2.9307 & 1 \\
\hline 2 & 3.5500 & 3.5700 & 2 & 18 & 2.9326 & 2.9384 & 1 \\
\hline 3 & 3.3800 & 3.3900 & 2 & 19 & 3.1300 & 3.1300 & 1 \\
\hline 3 & 3.3900 & 3.3900 & 2 & 19 & 3.1600 & 3.1600 & 1 \\
\hline 3 & 3.3900 & 3.4000 & 2 & 19 & 3.1500 & 3.1600 & 1 \\
\hline 3 & 3.3800 & 3.3900 & 2 & 19 & 3.1800 & 3.1800 & 1 \\
\hline 3 & 3.3700 & 3.3700 & 2 & 19 & 3.1700 & 3.1600 & 1 \\
\hline 3 & 3.4200 & 3.4100 & 2 & 19 & 3.1200 & 3.1200 & 1 \\
\hline 4 & 3.5230 & 3.5140 & 2 & 20 & 2.9200 & 2.9200 & 1 \\
\hline 4 & 3.5010 & 3.4950 & 2 & 20 & 2.9000 & 2.8900 & 1 \\
\hline 4 & 3.5380 & 3.5560 & 2 & 20 & 2.9100 & 2.9100 & 1 \\
\hline 4 & 3.5160 & 3.5170 & 2 & 20 & 2.9000 & 2.9000 & 1 \\
\hline 4 & 3.5120 & 3.5190 & 2 & 20 & 2.9200 & 2.9200 & 1 \\
\hline 4 & 3.5240 & 3.5330 & 2 & 20 & 2.9300 & 2.9200 & 1 \\
\hline 5 & 3.6090 & 3.5940 & 2 & 21 & 2.9361 & 2.9412 & 1 \\
\hline 5 & 3.6340 & 3.6270 & 2 & 21 & 2.9403 & 2.9359 & 1 \\
\hline 5 & 3.6030 & 3.5960 & 2 & 21 & 2.9161 & 2.9142 & 1 \\
\hline 5 & 3.5110 & 3.4950 & 2 & 21 & 2.9321 & 2.9334 & 1 \\
\hline 5 & 3.5920 & 3.5840 & 2 & 21 & 2.9098 & 2.9126 & 1 \\
\hline 5 & 3.6110 & 3.5930 & 2 & 21 & 2.9211 & 2.9187 & 1 \\
\hline 6 & 3.5410 & 3.5410 & 2 & 22 & 2.9300 & 2.9300 & 1 \\
\hline 6 & 3.5180 & 3.5430 & 2 & 22 & 2.9300 & 2.9400 & 1 \\
\hline 6 & 3.5420 & 3.5400 & 2 & 22 & 2.9700 & 2.9700 & 1 \\
\hline 6 & 3.5210 & 3.5520 & 2 & 22 & 2.9700 & 2.9800 & 1 \\
\hline 6 & 3.5450 & 3.5520 & 2 & 22 & 2.9500 & 2.9500 & 1 \\
\hline 6 & 3.5530 & 3.5800 & 2 & 22 & 2.9600 & 2.9600 & 1 \\
\hline 7 & 3.6480 & 3.6570 & 2 & 23 & 2.9600 & 2.9300 & 1 \\
\hline 7 & 3.5740 & 3.5680 & 2 & 23 & 2.9200 & 2.9000 & 1 \\
\hline 7 & 3.5760 & 3.5750 & 2 & 23 & 2.9500 & 2.9100 & 1 \\
\hline 7 & 3.6520 & 3.6560 & 2 & 23 & 2.9400 & 2.9100 & 1 \\
\hline 7 & 3.6500 & 3.6520 & 2 & 23 & 2.9200 & 2.9200 & 1 \\
\hline 7 & 3.6620 & 3.6620 & 2 & 23 & 2.9000 & 2.9100 & 1 \\
\hline 8 & 3.6700 & 3.6500 & 2 & 24 & 2.7300 & 2.6900 & 1 \\
\hline 8 & 3.6700 & 3.6400 & 2 & 24 & 2.6700 & 2.6600 & 1 \\
\hline 8 & 3.6500 & 3.6600 & 2 & 24 & 2.6300 & 2.6300 & 1 \\
\hline 8 & 3.6200 & 3.6400 & 2 & 24 & 2.6700 & 2.6600 & 1 \\
\hline 8 & 3.6300 & 3.6500 & 2 & 24 & 2.7000 & 2.7000 & 1 \\
\hline 8 & 3.6500 & 3.6500 & 2 & 24 & 2.6800 & 2.6700 & 1 \\
\hline
\end{tabular}




\begin{tabular}{|c|c|c|c|c|c|c|c|}
\hline 9 & 3.6100 & 3.6390 & 2 & 1 & 3.4500 & 3.4500 & 2 \\
\hline 9 & 3.6480 & 3.6450 & 2 & 1 & 3.4500 & 3.4500 & 2 \\
\hline 9 & 3.6020 & 3.5750 & 2 & 1 & 3.4700 & 3.4800 & 2 \\
\hline 9 & 3.6110 & 3.5970 & 2 & 1 & 3.4400 & 3.4400 & 2 \\
\hline 9 & 3.5890 & 3.6150 & 2 & 1 & 3.4700 & 3.4700 & 2 \\
\hline 9 & 3.5940 & 3.5930 & 2 & 1 & 3.4700 & 3.4700 & 2 \\
\hline 10 & 3.4670 & 3.4650 & 2 & 2 & 3.3800 & 3.3900 & 2 \\
\hline 10 & 3.4670 & 3.4650 & 2 & 2 & 3.3900 & 3.4100 & 2 \\
\hline 10 & 3.4750 & 3.4740 & 2 & 2 & 3.3900 & 3.4100 & 2 \\
\hline 10 & 3.3730 & 3.3770 & 2 & 2 & 3.3700 & 3.3900 & 2 \\
\hline 10 & 3.3730 & 3.3770 & 2 & 2 & 3.3900 & 3.4000 & 2 \\
\hline 10 & 3.4430 & 3.4450 & 2 & 2 & 3.3900 & 3.3900 & 2 \\
\hline 11 & 3.6580 & 3.6690 & 2 & 3 & 3.5700 & 3.5800 & 2 \\
\hline 11 & 3.6620 & 3.6520 & 2 & 3 & 3.5600 & 3.5600 & 2 \\
\hline 11 & 3.6730 & 3.6630 & 2 & 3 & 3.5600 & 3.5500 & 2 \\
\hline 11 & 3.6800 & 3.6760 & 2 & 3 & 3.5900 & 3.5800 & 2 \\
\hline 11 & 3.6810 & 3.6730 & 2 & 3 & 3.5800 & 3.6000 & 2 \\
\hline 11 & 3.6790 & 3.6800 & 2 & 3 & 3.5800 & 3.5800 & 2 \\
\hline 12 & 3.5700 & 2.5800 & 2 & 4 & 3.5600 & 3.5800 & 2 \\
\hline 12 & 3.5400 & 3.5400 & 2 & 4 & 3.5800 & 3.5800 & 2 \\
\hline 12 & 3.5400 & 3.5800 & 2 & 4 & 3.5900 & 3.5900 & 2 \\
\hline 12 & 3.6100 & 3.6000 & 2 & 4 & 3.5800 & 3.5900 & 2 \\
\hline 12 & 3.6000 & 3.5500 & 2 & 4 & 3.6000 & 3.6100 & 2 \\
\hline 12 & 3.5600 & 3.5800 & 2 & 4 & 3.5900 & 3.5900 & 2 \\
\hline 13 & 3.6000 & 3.6000 & 2 & 5 & 3.5810 & 3.5870 & 2 \\
\hline 13 & 3.6000 & 3.6000 & 2 & 5 & 3.6290 & 3.6210 & 2 \\
\hline 13 & 3.6200 & 3.6200 & 2 & 5 & 3.6280 & 3.6340 & 2 \\
\hline 13 & 3.6300 & 3.6100 & 2 & 5 & 3.5900 & 3.5890 & 2 \\
\hline 13 & 3.6500 & 3.6500 & 2 & 5 & 3.6370 & 3.6370 & 2 \\
\hline 13 & 3.6200 & 3.6300 & 2 & 5 & 3.6080 & 3.6090 & 2 \\
\hline 14 & 3.6400 & 3.6400 & 2 & 6 & 3.4900 & 3.4900 & 2 \\
\hline 14 & 3.6400 & 3.6500 & 2 & 6 & 3.5500 & 3.5600 & 2 \\
\hline 14 & 3.6300 & 3.6300 & 2 & 6 & 3.5400 & 3.5700 & 2 \\
\hline 14 & 3.6400 & 3.6300 & 2 & 6 & 3.5200 & 3.5300 & 2 \\
\hline 14 & 3.6300 & 3.6400 & 2 & 6 & 3.5600 & 3.5500 & 2 \\
\hline 14 & 3.6300 & 3.6300 & 2 & 6 & 3.5400 & 3.5700 & 2 \\
\hline 15 & 3.5300 & 3.5300 & 2 & 7 & 3.5830 & 3.5880 & 2 \\
\hline 15 & 3.5400 & 3.5400 & 2 & 7 & 3.6060 & 3.6160 & 2 \\
\hline 15 & 3.5300 & 3.5200 & 2 & 7 & 3.6180 & 3.6110 & 2 \\
\hline 15 & 3.5300 & 3.5300 & 2 & 7 & 3.6840 & 3.6790 & 2 \\
\hline 15 & 3.5100 & 3.5000 & 2 & 7 & 3.6760 & 3.6720 & 2 \\
\hline 15 & 3.5300 & 3.5100 & 2 & 7 & 3.6050 & 3.5960 & 2 \\
\hline 16 & 3.6000 & 3.5800 & 2 & 8 & 3.7500 & 3.7500 & 2 \\
\hline 16 & 3.6000 & 3.6000 & 2 & 8 & 3.7000 & 3.7000 & 2 \\
\hline 16 & 3.5900 & 3.5900 & 2 & 8 & 3.7300 & 3.7300 & 2 \\
\hline 16 & 3.5700 & 3.5800 & 2 & 8 & 3.7600 & 3.7600 & 2 \\
\hline 16 & 3.5500 & 3.5600 & 2 & 8 & 3.7000 & 3.7000 & 2 \\
\hline \multirow[t]{2}{*}{16} & 3.5800 & 3.5800 & 2 & 8 & 3.7500 & 3.7500 & 2 \\
\hline & & & & 9 & 3.3060 & 3.2990 & 2 \\
\hline
\end{tabular}




$\begin{array}{rlll}9 & 3.3060 & 3.2990 & 2 \\ 9 & 3.3350 & 3.3350 & 2 \\ 9 & 3.2970 & 3.2910 & 2 \\ 9 & 3.2970 & 3.2910 & 2 \\ 9 & 3.3100 & 3.3030 & 2 \\ 10 & 3.6100 & 3.6000 & 2 \\ 10 & 3.6300 & 3.6100 & 2 \\ 10 & 3.6100 & 3.6300 & 2 \\ 10 & 3.6000 & 3.6100 & 2 \\ 10 & 3.6500 & 3.6400 & 2 \\ 10 & 3.6300 & 3.6300 & 2 \\ 11 & 3.6690 & 3.6550 & 2 \\ 11 & 3.7550 & 3.7510 & 2 \\ 11 & 3.6560 & 3.6670 & 2 \\ 11 & 3.7360 & 3.7320 & 2 \\ 11 & 3.6980 & 3.7000 & 2 \\ 11 & 3.6960 & 3.6980 & 2 \\ 12 & 3.6010 & 3.6120 & 2 \\ 12 & 3.5990 & 3.6030 & 2 \\ 12 & 3.6050 & 3.6150 & 2 \\ 12 & 3.6150 & 3.6140 & 2 \\ 12 & 3.6210 & 3.6140 & 2 \\ 12 & 3.6110 & 3.6220 & 2 \\ 13 & 3.4300 & 3.4300 & 2 \\ 13 & 3.5000 & 3.5000 & 2 \\ 13 & 3.4600 & 3.4600 & 2 \\ 13 & 3.4600 & 3.4500 & 2 \\ 13 & 3.4800 & 3.4900 & 2 \\ 13 & 3.4100 & 3.4200 & 2 \\ 14 & 3.5900 & 3.5960 & 2 \\ 14 & 3.6090 & 3.6130 & 2 \\ 14 & 3.6060 & 3.6050 & 2 \\ 14 & 3.5440 & 3.5490 & 2 \\ 14 & 3.5750 & 3.5720 & 2 \\ 14 & 3.5770 & 3.5800 & 2 \\ 15 & 3.6100 & 3.6100 & 2 \\ 15 & 3.6200 & 3.6000 & 2 \\ 15 & 3.6300 & 3.6200 & 2 \\ 15 & 3.5900 & 3.5900 & 2 \\ 15 & 3.6200 & 3.6100 & 2 \\ 15 & 3.6600 & 3.6300 & 2 \\ 16 & 3.6100 & 3.6000 & 2 \\ 16 & 3.6300 & 3.6300 & 2 \\ 16 & 3.6300 & 3.6300 & 2 \\ 16 & 3.6000 & 3.6100 & 2 \\ 16 & 3.6100 & 3.6300 & 2 \\ 16 & 3.6200 & 3.6100 & 2 \\ 17 & 3.1700 & 3.1700 & 2 \\ 17 & 3.1700 & 3.1700 & 2\end{array}$




$\begin{array}{llll}17 & 3.1400 & 3.1300 & 2 \\ 17 & 3.1600 & 3.1600 & 2 \\ 17 & 3.2100 & 3.2100 & 2 \\ 17 & 3.1400 & 3.1300 & 2 \\ 18 & 3.5657 & 3.5690 & 2 \\ 18 & 3.6025 & 3.6051 & 2 \\ 18 & 3.6097 & 3.6157 & 2 \\ 18 & 3.5876 & 3.5892 & 2 \\ 18 & 3.6617 & 3.6620 & 2 \\ 18 & 3.6617 & 3.6621 & 2 \\ 19 & 3.7800 & 3.7800 & 2 \\ 19 & 3.7700 & 3.7700 & 2 \\ 19 & 3.7800 & 3.7900 & 2 \\ 19 & 3.8100 & 3.8100 & 2 \\ 19 & 3.8000 & 3.8000 & 2 \\ 19 & 3.7900 & 3.7800 & 2 \\ 20 & 3.5700 & 3.5700 & 2 \\ 20 & 3.6300 & 3.6200 & 2 \\ 20 & 3.5700 & 3.5800 & 2 \\ 20 & 3.6000 & 3.6100 & 2 \\ 20 & 3.6300 & 3.6300 & 2 \\ 20 & 3.6300 & 3.6200 & 2 \\ 21 & 3.5468 & 3.5474 & 2 \\ 21 & 3.5463 & 3.5475 & 2 \\ 21 & 3.5569 & 3.5597 & 2 \\ 21 & 3.5234 & 3.5215 & 2 \\ 21 & 3.5385 & 3.5453 & 2 \\ 21 & 3.5551 & 3.5583 & 2 \\ 22 & 3.5200 & 3.5200 & 2 \\ 22 & 3.5500 & 3.5500 & 2 \\ 22 & 3.5200 & 3.5200 & 2 \\ 22 & 3.5600 & 3.5500 & 2 \\ 22 & 3.5800 & 3.5700 & 2 \\ 22 & 3.5400 & 3.5400 & 2 \\ 23 & 3.6000 & 3.5800 & 2 \\ 23 & 3.5800 & 3.5700 & 2 \\ 23 & 3.6500 & 3.6100 & 2 \\ 23 & 3.5600 & 3.5900 & 2 \\ 23 & 3.5400 & 3.5300 & 2 \\ 23 & 3.6000 & 3.6000 & 2 \\ 24 & 3.2700 & 3.3400 & 2 \\ 24 & 3.3800 & 3.3800 & 2 \\ 24 & 3.4000 & 3.4000 & 2 \\ 24 & 3.3900 & 3.3900 & 2 \\ 24 & 3.2900 & 3.3000 & 2 \\ 24 & 3.3300 & 3.3600 & 2\end{array}$




\begin{tabular}{|c|c|c|c|c|c|c|c|}
\hline \multicolumn{4}{|c|}{ Na2O: Glass Preparation } & \multicolumn{4}{|c|}{ Na2O: Powder Preparation } \\
\hline Lab & Duplicate 1 & Duplicate 2 & Cement & Lab & Duplicate 1 & Duplicate 2 & Cement \\
\hline 1 & 0.2000 & 0.1900 & 1 & 1 & 0.2000 & 0.2000 & 1 \\
\hline 1 & 0.2000 & 0.2000 & 1 & 1 & 0.2000 & 0.2000 & 1 \\
\hline 1 & 0.1900 & 0.2000 & 1 & 1 & 0.2000 & 0.2000 & 1 \\
\hline 1 & 0.2000 & 0.1800 & 1 & 1 & 0.2000 & 0.2000 & 1 \\
\hline 1 & 0.1900 & 0.1900 & 1 & 1 & 0.2000 & 0.2000 & 1 \\
\hline 1 & 0.1900 & 0.2100 & 1 & 1 & 0.2000 & 0.2000 & 1 \\
\hline 2 & 0.2000 & 0.1900 & 1 & 2 & 0.1900 & 0.1900 & 1 \\
\hline 2 & 0.2000 & 0.2000 & 1 & 2 & 0.1800 & 0.1800 & 1 \\
\hline 2 & 0.2000 & 0.2000 & 1 & 2 & 0.1800 & 0.1800 & 1 \\
\hline 2 & 0.2000 & 0.2100 & 1 & 2 & 0.1800 & 0.1900 & 1 \\
\hline 2 & 0.1900 & 0.2000 & 1 & 2 & 0.1800 & 0.1800 & 1 \\
\hline 2 & 0.2000 & 0.2000 & 1 & 2 & 0.1800 & 0.1800 & 1 \\
\hline 3 & 0.2400 & 0.2300 & 1 & 3 & 0.1760 & 0.1740 & 1 \\
\hline 3 & 0.2500 & 0.2600 & 1 & 3 & 0.1740 & 0.1790 & 1 \\
\hline 3 & 0.2200 & 0.2400 & 1 & 3 & 0.1770 & 0.1780 & 1 \\
\hline 3 & 0.2500 & 0.2200 & 1 & 3 & 0.1800 & 0.1800 & 1 \\
\hline 3 & 0.2200 & 0.2300 & 1 & 3 & 0.1780 & 0.1770 & 1 \\
\hline 3 & 0.2300 & 0.2300 & 1 & 3 & 0.1790 & 0.1820 & 1 \\
\hline 4 & 0.2000 & 0.2200 & 1 & 4 & 0.1600 & 0.1800 & 1 \\
\hline 4 & 0.2200 & 0.2100 & 1 & 4 & 0.1900 & 0.1900 & 1 \\
\hline 4 & 0.2200 & 0.2100 & 1 & 4 & 0.1900 & 0.1900 & 1 \\
\hline 4 & 0.2000 & 0.2100 & 1 & 4 & 0.1800 & 0.1900 & 1 \\
\hline 4 & 0.2000 & 0.2000 & 1 & 4 & 0.1800 & 0.1800 & 1 \\
\hline 4 & 0.2100 & 0.2100 & 1 & 4 & 0.1900 & 0.1900 & 1 \\
\hline 5 & 0.2090 & 0.2040 & 1 & 5 & 0.1989 & 0.2009 & 1 \\
\hline 5 & 0.1980 & 0.2020 & 1 & 5 & 0.2029 & 0.2001 & 1 \\
\hline 5 & 0.2100 & 0.1980 & 1 & 5 & 0.1984 & 0.2008 & 1 \\
\hline 5 & 0.2010 & 0.2040 & 1 & 5 & 0.1969 & 0.1982 & 1 \\
\hline 5 & 0.1970 & 0.2040 & 1 & 5 & 0.2140 & 0.2157 & 1 \\
\hline 5 & 0.1990 & 0.2020 & 1 & 5 & 0.2051 & 0.2042 & 1 \\
\hline 6 & 0.2102 & 0.2093 & 1 & 6 & 0.2000 & 0.2000 & 1 \\
\hline 6 & 0.2103 & 0.2106 & 1 & 6 & 0.2000 & 0.2000 & 1 \\
\hline 6 & 0.2103 & 0.2093 & 1 & 6 & 0.2000 & 0.2000 & 1 \\
\hline 6 & 0.2099 & 0.2111 & 1 & 6 & 0.2000 & 0.2000 & 1 \\
\hline 6 & 0.2107 & 0.2095 & 1 & 6 & 0.2000 & 0.2000 & 1 \\
\hline 6 & 0.2113 & 0.2112 & 1 & 6 & 0.2000 & 0.2000 & 1 \\
\hline 7 & 0.1800 & 0.2000 & 1 & 7 & 0.1600 & 0.1500 & 1 \\
\hline 7 & 0.1900 & 0.1800 & 1 & 7 & 0.1500 & 0.1500 & 1 \\
\hline 7 & 0.2000 & 0.1800 & 1 & 7 & 0.1500 & 0.1600 & 1 \\
\hline 7 & 0.1900 & 0.2100 & 1 & 7 & 0.1500 & 0.1500 & 1 \\
\hline 7 & 0.1800 & 0.1900 & 1 & 7 & 0.1500 & 0.1500 & 1 \\
\hline 7 & 0.1800 & 0.1800 & 1 & 7 & 0.1500 & 0.1500 & 1 \\
\hline 8 & 0.2150 & 0.2200 & 1 & 8 & 0.2100 & 0.2100 & 1 \\
\hline 8 & 0.2180 & 0.2270 & 1 & 8 & 0.2100 & 0.2100 & 1 \\
\hline 8 & 0.2270 & 0.2280 & 1 & 8 & 0.2100 & 0.2100 & 1 \\
\hline 8 & 0.2250 & 0.2110 & 1 & 8 & 0.2100 & 0.2100 & 1 \\
\hline
\end{tabular}




\begin{tabular}{|c|c|c|c|c|c|c|}
\hline 8 & 0.2130 & 0.2210 & 1 & 8 & 0.2100 & 0.2100 \\
\hline 8 & 0.2110 & 0.2070 & 1 & 8 & 0.2100 & 0.2100 \\
\hline 9 & 0.2200 & 0.2300 & 1 & 9 & 0.1900 & 0.1900 \\
\hline 9 & 0.2400 & 0.2300 & 1 & 9 & 0.1800 & 0.1900 \\
\hline 9 & 0.2300 & 0.2100 & 1 & 9 & 0.1900 & 0.1900 \\
\hline 9 & 0.2100 & 0.2200 & 1 & 9 & 0.1900 & 0.1900 \\
\hline 9 & 0.2300 & 0.2400 & 1 & 9 & 0.1900 & 0.1900 \\
\hline 9 & 0.2500 & 0.2400 & 1 & 9 & 0.1900 & 0.1900 \\
\hline 10 & 0.2073 & 0.2181 & 1 & 10 & 0.2000 & 0.2000 \\
\hline 10 & 0.2075 & 0.2116 & 1 & 10 & 0.1900 & 0.1900 \\
\hline 10 & 0.2086 & 0.2123 & 1 & 10 & 0.2000 & 0.2000 \\
\hline 10 & 0.1985 & 0.2127 & 1 & 10 & 0.2100 & 0.2100 \\
\hline 10 & 0.2064 & 0.2090 & 1 & 10 & 0.2000 & 0.2000 \\
\hline 10 & 0.2054 & 0.2080 & 1 & 10 & 0.2000 & 0.2000 \\
\hline 11 & 0.2120 & 0.2060 & 1 & 11 & 0.2000 & 0.2000 \\
\hline 11 & 0.2150 & 0.2070 & 1 & 11 & 0.2100 & 0.2000 \\
\hline 11 & 0.2150 & 0.2020 & 1 & 11 & 0.2100 & 0.2000 \\
\hline 11 & 0.2040 & 0.2160 & 1 & 11 & 0.2100 & 0.2100 \\
\hline 11 & 0.2080 & 0.2030 & 1 & 11 & 0.2100 & 0.2100 \\
\hline 11 & 0.2040 & 0.2000 & 1 & 11 & 0.2100 & 0.2100 \\
\hline 12 & 0.2100 & 0.2200 & 1 & 12 & 0.2030 & 0.2040 \\
\hline 12 & 0.2100 & 0.2000 & 1 & 12 & 0.2050 & 0.2050 \\
\hline 12 & 0.2200 & 0.2100 & 1 & 12 & 0.2050 & 0.2060 \\
\hline 12 & 0.2200 & 0.2000 & 1 & 12 & 0.2060 & 0.2080 \\
\hline 12 & 0.2200 & 0.2100 & 1 & 12 & 0.2070 & 0.2060 \\
\hline 12 & 0.2000 & 0.2100 & 1 & 12 & 0.2070 & 0.2060 \\
\hline 13 & 0.2550 & 0.2360 & 1 & 13 & 0.2030 & 0.2040 \\
\hline 13 & 0.2430 & 0.2230 & 1 & 13 & 0.2070 & 0.2080 \\
\hline 13 & 0.2140 & 0.2040 & 1 & 13 & 0.2080 & 0.2110 \\
\hline 13 & 0.2080 & 0.2220 & 1 & 13 & 0.2070 & 0.2060 \\
\hline 13 & 0.2350 & 0.2200 & 1 & 13 & 0.2020 & 0.2070 \\
\hline 13 & 0.2210 & 0.2290 & 1 & 13 & 0.2060 & 0.2080 \\
\hline 14 & 0.2200 & 0.2170 & 1 & 14 & 0.1764 & 0.1771 \\
\hline 14 & 0.2080 & 0.2140 & 1 & 14 & 0.1767 & 0.1768 \\
\hline 14 & 0.2140 & 0.2120 & 1 & 14 & 0.1765 & 0.1766 \\
\hline 14 & 0.2070 & 0.2080 & 1 & 14 & 0.1766 & 0.1763 \\
\hline 14 & 0.2070 & 0.2100 & 1 & 14 & 0.1789 & 0.1768 \\
\hline 14 & 0.2130 & 0.2080 & 1 & 14 & 0.1762 & 0.1765 \\
\hline 15 & 0.1920 & 0.2020 & 1 & 15 & 0.1480 & 0.1480 \\
\hline 15 & 0.1950 & 0.2070 & 1 & 15 & 0.1470 & 0.1470 \\
\hline 15 & 0.2000 & 0.1900 & 1 & 15 & 0.1380 & 0.1500 \\
\hline 15 & 0.1990 & 0.1900 & 1 & 15 & 0.1510 & 0.1500 \\
\hline 15 & 0.1940 & 0.1910 & 1 & 15 & 0.1500 & 0.1470 \\
\hline 15 & 0.1790 & 0.2000 & 1 & 15 & 0.1510 & 0.1520 \\
\hline 1 & 0.0400 & 0.0400 & 2 & 16 & 0.1800 & 0.1800 \\
\hline 1 & 0.0600 & 0.0600 & 2 & 16 & 0.1800 & 0.1900 \\
\hline 1 & 0.0400 & 0.0400 & 2 & 16 & 0.1900 & 0.1900 \\
\hline 1 & 0.0300 & 0.0400 & 2 & 16 & 0.1800 & 0.1800 \\
\hline 1 & 0.0300 & 0.0500 & 2 & 16 & 0.1800 & 0.1800 \\
\hline
\end{tabular}




\begin{tabular}{|c|c|c|c|c|c|c|}
\hline 1 & 0.0500 & 0.0400 & 2 & 16 & 0.1800 & 0.1900 \\
\hline 2 & 0.0700 & 0.0500 & 2 & 17 & 0.1550 & 0.1680 \\
\hline 2 & 0.0600 & 0.0600 & 2 & 17 & 0.1640 & 0.1460 \\
\hline 2 & 0.0600 & 0.0700 & 2 & 17 & 0.1600 & 0.1540 \\
\hline 2 & 0.0500 & 0.0600 & 2 & 17 & 0.1560 & 0.1520 \\
\hline 2 & 0.0600 & 0.0500 & 2 & 17 & 0.1470 & 0.1540 \\
\hline 2 & 0.0700 & 0.0600 & 2 & 17 & 0.1470 & 0.1430 \\
\hline 3 & 0.0900 & 0.0900 & 2 & 18 & 0.1770 & 0.1760 \\
\hline 3 & 0.0900 & 0.1400 & 2 & 18 & 0.1730 & 0.1730 \\
\hline 3 & 0.1100 & 0.1100 & 2 & 18 & 0.1800 & 0.1780 \\
\hline 3 & 0.1100 & 0.1000 & 2 & 18 & 0.1710 & 0.1710 \\
\hline 3 & 0.0900 & 0.1000 & 2 & 18 & 0.1790 & 0.1820 \\
\hline 3 & 0.0900 & 0.1000 & 2 & 18 & 0.1750 & 0.1740 \\
\hline 4 & 0.0500 & 0.0500 & 2 & 19 & 0.2240 & 0.2390 \\
\hline 4 & 0.0500 & 0.0500 & 2 & 19 & 0.2060 & 0.2030 \\
\hline 4 & 0.0500 & 0.0600 & 2 & 19 & 0.2090 & 0.2120 \\
\hline 4 & 0.0400 & 0.0300 & 2 & 19 & 0.2100 & 0.2170 \\
\hline 4 & 0.0400 & 0.0500 & 2 & 19 & 0.2040 & 0.2040 \\
\hline 4 & 0.0400 & 0.0400 & 2 & 19 & 0.2080 & 0.2110 \\
\hline 5 & 0.0580 & 0.0560 & 2 & 20 & 0.1800 & 0.1800 \\
\hline 5 & 0.0590 & 0.0550 & 2 & 20 & 0.1700 & 0.1700 \\
\hline 5 & 0.0620 & 0.0600 & 2 & 20 & 0.1800 & 0.1800 \\
\hline 5 & 0.0580 & 0.0600 & 2 & 20 & 0.1600 & 0.1600 \\
\hline 5 & 0.0610 & 0.0570 & 2 & 20 & 0.1600 & 0.1600 \\
\hline 5 & 0.0540 & 0.0540 & 2 & 20 & 0.1600 & 0.1700 \\
\hline 6 & 0.0560 & 0.0565 & 2 & 21 & 0.1860 & 0.1760 \\
\hline 6 & 0.0560 & 0.0565 & 2 & 21 & 0.1900 & 0.1850 \\
\hline 6 & 0.0557 & 0.0556 & 2 & 21 & 0.1850 & 0.1920 \\
\hline 6 & 0.0553 & 0.0553 & 2 & 21 & 0.1930 & 0.1930 \\
\hline 6 & 0.0553 & 0.0553 & 2 & 21 & 0.1920 & 0.1910 \\
\hline 6 & 0.0555 & 0.0549 & 2 & 21 & 0.1800 & 0.1850 \\
\hline 7 & 0.0000 & 0.0000 & 2 & 22 & 0.2090 & 0.2080 \\
\hline 7 & 0.0000 & 0.0000 & 2 & 22 & 0.2130 & 0.2130 \\
\hline 7 & 0.0000 & 0.0000 & 2 & 22 & 0.2080 & 0.2050 \\
\hline 7 & 0.0000 & 0.0000 & 2 & 22 & 0.2110 & 0.2070 \\
\hline 7 & 0.0000 & 0.0000 & 2 & 22 & 0.2120 & 0.2100 \\
\hline 7 & 0.0000 & 0.0000 & 2 & 22 & 0.2100 & 0.2120 \\
\hline 8 & 0.0750 & 0.0750 & 2 & 23 & 0.1694 & 0.1635 \\
\hline 8 & 0.0700 & 0.0690 & 2 & 23 & 0.1669 & 0.1600 \\
\hline 8 & 0.0700 & 0.0680 & 2 & 23 & 0.1695 & 0.1664 \\
\hline 8 & 0.0690 & 0.0680 & 2 & 23 & 0.1702 & 0.1695 \\
\hline 8 & 0.0700 & 0.0710 & 2 & 23 & 0.1667 & 0.1682 \\
\hline 8 & 0.0720 & 0.0690 & 2 & 23 & 0.1680 & 0.1717 \\
\hline 9 & 0.0900 & 0.0900 & 2 & 24 & 0.1800 & 0.1800 \\
\hline 9 & 0.1000 & 0.0900 & 2 & 24 & 0.1700 & 0.1800 \\
\hline 9 & 0.0700 & 0.0900 & 2 & 24 & 0.1700 & 0.1800 \\
\hline 9 & 0.0900 & 0.1000 & 2 & 24 & 0.1800 & 0.1800 \\
\hline 9 & 0.0900 & 0.0900 & 2 & 24 & 0.1700 & 0.1700 \\
\hline 9 & 0.1000 & 0.1000 & 2 & 24 & 0.1700 & 0.1700 \\
\hline
\end{tabular}




\begin{tabular}{|c|c|c|c|c|c|c|}
\hline 10 & 0.0465 & 0.0448 & 2 & 1 & 0.0500 & 0.0500 \\
\hline 10 & 0.0452 & 0.0446 & 2 & 1 & 0.0500 & 0.0500 \\
\hline 10 & 0.0510 & 0.0467 & 2 & 1 & 0.0500 & 0.0500 \\
\hline 10 & 0.0407 & 0.0502 & 2 & 1 & 0.0600 & 0.0600 \\
\hline 10 & 0.0487 & 0.0485 & 2 & 1 & 0.0600 & 0.0600 \\
\hline 10 & 0.0413 & 0.0482 & 2 & 1 & 0.0600 & 0.0600 \\
\hline 11 & 0.0410 & 0.0450 & 2 & 2 & 0.0500 & 0.0500 \\
\hline 11 & 0.0450 & 0.0330 & 2 & 2 & 0.0500 & 0.0500 \\
\hline 11 & 0.0480 & 0.0570 & 2 & 2 & 0.0500 & 0.0500 \\
\hline 11 & 0.0280 & 0.0490 & 2 & 2 & 0.0500 & 0.0600 \\
\hline 11 & 0.0470 & 0.0420 & 2 & 2 & 0.0600 & 0.0600 \\
\hline 11 & 0.0420 & 0.0420 & 2 & 2 & 0.0500 & 0.0500 \\
\hline 12 & 0.0600 & 0.0600 & 2 & 3 & 0.0540 & 0.0550 \\
\hline 12 & 0.0600 & 0.0600 & 2 & 3 & 0.0530 & 0.0560 \\
\hline 12 & 0.0600 & 0.0600 & 2 & 3 & 0.0550 & 0.0540 \\
\hline 12 & 0.0600 & 0.0600 & 2 & 3 & 0.0590 & 0.0650 \\
\hline 12 & 0.0700 & 0.0700 & 2 & 3 & 0.0650 & 0.0660 \\
\hline 12 & 0.0700 & 0.0700 & 2 & 3 & 0.0640 & 0.0660 \\
\hline 13 & 0.0490 & 0.0770 & 2 & 4 & 0.0700 & 0.0700 \\
\hline 13 & 0.0530 & 0.0580 & 2 & 4 & 0.0700 & 0.0600 \\
\hline 13 & 0.0700 & 0.0530 & 2 & 4 & 0.0600 & 0.0600 \\
\hline 13 & 0.0840 & 0.0780 & 2 & 4 & 0.0700 & 0.0700 \\
\hline 13 & 0.0760 & 0.1050 & 2 & 4 & 0.0600 & 0.0600 \\
\hline 13 & 0.0860 & 0.0950 & 2 & 4 & 0.0600 & 0.0600 \\
\hline 14 & 0.0650 & 0.0650 & 2 & 5 & 0.0656 & 0.0657 \\
\hline 14 & 0.0680 & 0.0670 & 2 & 5 & 0.0627 & 0.0604 \\
\hline 14 & 0.0610 & 0.0630 & 2 & 5 & 0.0639 & 0.0624 \\
\hline 14 & 0.0620 & 0.0610 & 2 & 5 & 0.0750 & 0.0759 \\
\hline 14 & 0.0640 & 0.0620 & 2 & 5 & 0.0730 & 0.0731 \\
\hline 14 & 0.0700 & 0.0680 & 2 & 5 & 0.0722 & 0.0738 \\
\hline 15 & 0.0490 & 0.0550 & 2 & 6 & 0.0700 & 0.0700 \\
\hline 15 & 0.0500 & 0.0500 & 2 & 6 & 0.0700 & 0.0700 \\
\hline 15 & 0.0640 & 0.0670 & 2 & 6 & 0.0700 & 0.0700 \\
\hline 15 & 0.0460 & 0.0350 & 2 & 6 & 0.0700 & 0.0700 \\
\hline 15 & 0.0430 & 0.0330 & 2 & 6 & 0.0700 & 0.0700 \\
\hline \multirow[t]{14}{*}{15} & 0.0370 & 0.0430 & 2 & 6 & 0.0700 & 0.0700 \\
\hline & & & & 7 & 0.0200 & 0.0200 \\
\hline & & & & 7 & 0.0200 & 0.0200 \\
\hline & & & & 7 & 0.0200 & 0.0200 \\
\hline & & & & 7 & 0.0100 & 0.0100 \\
\hline & & & & 7 & 0.0100 & 0.0100 \\
\hline & & & & 7 & 0.0100 & 0.0100 \\
\hline & & & & 8 & 0.0600 & 0.0600 \\
\hline & & & & 8 & 0.0600 & 0.0600 \\
\hline & & & & 8 & 0.0600 & 0.0600 \\
\hline & & & & 8 & 0.0600 & 0.0600 \\
\hline & & & & 8 & 0.0600 & 0.0600 \\
\hline & & & & 8 & 0.0600 & 0.0600 \\
\hline & & & & 9 & 0.0400 & 0.0500 \\
\hline
\end{tabular}




$\begin{array}{rlll}9 & 0.0500 & 0.0400 & 2 \\ 9 & 0.0400 & 0.0500 & 2 \\ 9 & 0.0500 & 0.0500 & 2 \\ 9 & 0.0400 & 0.0400 & 2 \\ 9 & 0.0500 & 0.0400 & 2 \\ 10 & 0.0500 & 0.0500 & 2 \\ 10 & 0.0500 & 0.0500 & 2 \\ 10 & 0.0500 & 0.0500 & 2 \\ 10 & 0.0600 & 0.0600 & 2 \\ 10 & 0.0500 & 0.0500 & 2 \\ 10 & 0.0500 & 0.0500 & 2 \\ 11 & 0.0800 & 0.0800 & 2 \\ 11 & 0.0800 & 0.0800 & 2 \\ 11 & 0.0700 & 0.0700 & 2 \\ 11 & 0.0800 & 0.0800 & 2 \\ 11 & 0.0700 & 0.0700 & 2 \\ 11 & 0.0700 & 0.0700 & 2 \\ 12 & 0.0690 & 0.0670 & 2 \\ 12 & 0.0680 & 0.0680 & 2 \\ 12 & 0.0660 & 0.0650 & 2 \\ 12 & 0.0660 & 0.0650 & 2 \\ 12 & 0.0670 & 0.0650 & 2 \\ 12 & 0.0650 & 0.0650 & 2 \\ 13 & 0.0810 & 0.0840 & 2 \\ 13 & 0.0830 & 0.0830 & 2 \\ 13 & 0.0840 & 0.0840 & 2 \\ 13 & 0.0850 & 0.0840 & 2 \\ 13 & 0.0880 & 0.0840 & 2 \\ 13 & 0.0850 & 0.0820 & 2 \\ 14 & 0.0455 & 0.0457 & 2 \\ 14 & 0.0455 & 0.0457 & 2 \\ 14 & 0.0455 & 0.0457 & 2 \\ 14 & 0.0461 & 0.0455 & 2 \\ 14 & 0.0461 & 0.0455 & 2 \\ 14 & 0.0454 & 0.0453 & 2 \\ 15 & 0.0130 & 0.0140 & 2 \\ 15 & 0.0090 & 0.0160 & 2 \\ 15 & 0.0090 & 0.0130 & 2 \\ 15 & 0.0050 & 0.0130 & 2 \\ 15 & 0.0040 & 0.0120 & 2 \\ 15 & 0.0130 & 0.0160 & 2 \\ 16 & 0.0300 & 0.0300 & 2 \\ 16 & 0.0300 & 0.0300 & 2 \\ 16 & 0.0200 & 0.0300 & 2 \\ 16 & 0.0300 & 0.0300 & 2 \\ 16 & 0.0300 & 0.0200 & 2 \\ 16 & 0.0100 & 0.0100 & 2 \\ 17 & 0.0540 & 0.0430 & 2 \\ 17 & 0.0660 & 0.0490 & 2\end{array}$




$\begin{array}{llll}17 & 0.0750 & 0.0380 & 2 \\ 17 & 0.0360 & 0.0240 & 2 \\ 17 & 0.0320 & 0.0270 & 2 \\ 17 & 0.0260 & 0.0120 & 2 \\ 18 & 0.0420 & 0.0480 & 2 \\ 18 & 0.0420 & 0.0440 & 2 \\ 18 & 0.0440 & 0.0440 & 2 \\ 18 & 0.0430 & 0.0440 & 2 \\ 18 & 0.0380 & 0.0420 & 2 \\ 18 & 0.0390 & 0.0390 & 2 \\ 19 & 0.0620 & 0.0570 & 2 \\ 19 & 0.0690 & 0.0650 & 2 \\ 19 & 0.0480 & 0.0580 & 2 \\ 19 & 0.0530 & 0.0560 & 2 \\ 19 & 0.0560 & 0.0570 & 2 \\ 19 & 0.0600 & 0.0610 & 2 \\ 20 & 0.0500 & 0.0500 & 2 \\ 20 & 0.0400 & 0.0400 & 2 \\ 20 & 0.0400 & 0.0400 & 2 \\ 20 & 0.0500 & 0.0400 & 2 \\ 20 & 0.0500 & 0.0400 & 2 \\ 20 & 0.0500 & 0.0400 & 2 \\ 21 & 0.0740 & 0.0720 & 2 \\ 21 & 0.0740 & 0.0750 & 2 \\ 21 & 0.0640 & 0.0630 & 2 \\ 21 & 0.0780 & 0.0800 & 2 \\ 21 & 0.0710 & 0.0680 & 2 \\ 21 & 0.0740 & 0.0720 & 2 \\ 22 & 0.0640 & 0.0660 & 2 \\ 22 & 0.0580 & 0.0620 & 2 \\ 22 & 0.0630 & 0.0600 & 2 \\ 22 & 0.0600 & 0.0620 & 2 \\ 22 & 0.0650 & 0.0620 & 2 \\ 22 & 0.0660 & 0.0670 & 2 \\ 23 & 0.0461 & 0.0471 & 2 \\ 23 & 0.0461 & 0.0444 & 2 \\ 23 & 0.0443 & 0.0449 & 2 \\ 23 & 0.0512 & 0.0490 & 2 \\ 23 & 0.0507 & 0.0513 & 2 \\ 23 & 0.0488 & 0.0485 & 2 \\ 24 & 0.0400 & 0.0400 & 2 \\ 24 & 0.0400 & 0.0400 & 2 \\ 24 & 0.0400 & 0.0400 & 2 \\ 24 & 0.0400 & 0.0400 & 2 \\ 24 & 0.0400 & 0.0400 & 2 \\ 24 & 0.0400 & 0.0400 & 2\end{array}$




\begin{tabular}{|c|c|c|c|c|c|c|c|}
\hline \multicolumn{4}{|c|}{ K2O: Glass Preparation } & \multicolumn{4}{|c|}{ K2O: Powder Preparation } \\
\hline Lab & Duplicate 1 & Duplicate 2 & Cement & Lab & Duplicate 1 & Duplicate 2 & Cement \\
\hline 1 & 0.6800 & 0.6700 & 1 & 1 & 0.6360 & 0.6360 & 1 \\
\hline 1 & 0.6800 & 0.6800 & 1 & 1 & 0.6380 & 0.6370 & 1 \\
\hline 1 & 0.6800 & 0.6600 & 1 & 1 & 0.6380 & 0.6390 & 1 \\
\hline 1 & 0.6800 & 0.6800 & 1 & 1 & 0.6450 & 0.6440 & 1 \\
\hline 1 & 0.6800 & 0.6700 & 1 & 1 & 0.6400 & 0.6390 & 1 \\
\hline 1 & 0.6800 & 0.6800 & 1 & 1 & 0.6480 & 0.6470 & 1 \\
\hline 2 & 0.6800 & 0.6800 & 1 & 2 & 0.6740 & 0.6730 & 1 \\
\hline 2 & 0.6800 & 0.6800 & 1 & 2 & 0.6740 & 0.6730 & 1 \\
\hline 2 & 0.6800 & 0.6800 & 1 & 2 & 0.6730 & 0.6730 & 1 \\
\hline 2 & 0.6800 & 0.6800 & 1 & 2 & 0.6730 & 0.6710 & 1 \\
\hline 2 & 0.6800 & 0.6800 & 1 & 2 & 0.6720 & 0.6740 & 1 \\
\hline 2 & 0.6800 & 0.6800 & 1 & 2 & 0.6730 & 0.6730 & 1 \\
\hline 3 & 0.6920 & 0.6900 & 1 & 3 & 0.6600 & 0.6600 & 1 \\
\hline 3 & 0.6870 & 0.6940 & 1 & 3 & 0.6600 & 0.6500 & 1 \\
\hline 3 & 0.6890 & 0.6950 & 1 & 3 & 0.6600 & 0.6600 & 1 \\
\hline 3 & 0.6810 & 0.6860 & 1 & 3 & 0.6700 & 0.6600 & 1 \\
\hline 3 & 0.6860 & 0.6870 & 1 & 3 & 0.6600 & 0.6600 & 1 \\
\hline 3 & 0.6920 & 0.6880 & 1 & 3 & 0.6600 & 0.6600 & 1 \\
\hline 4 & 0.7000 & 0.7100 & 1 & 4 & 0.6980 & 0.6980 & 1 \\
\hline 4 & 0.7100 & 0.7100 & 1 & 4 & 0.7040 & 0.7040 & 1 \\
\hline 4 & 0.7100 & 0.7100 & 1 & 4 & 0.7060 & 0.7050 & 1 \\
\hline 4 & 0.7100 & 0.7000 & 1 & 4 & 0.7050 & 0.7050 & 1 \\
\hline 4 & 0.7100 & 0.7000 & 1 & 4 & 0.7000 & 0.7000 & 1 \\
\hline 4 & 0.7000 & 0.7100 & 1 & 4 & 0.7000 & 0.7010 & 1 \\
\hline 5 & 0.6900 & 0.6900 & 1 & 5 & 0.6800 & 0.6800 & 1 \\
\hline 5 & 0.7000 & 0.6900 & 1 & 5 & 0.6900 & 0.6700 & 1 \\
\hline 5 & 0.6900 & 0.7000 & 1 & 5 & 0.6700 & 0.6800 & 1 \\
\hline 5 & 0.6900 & 0.7000 & 1 & 5 & 0.6700 & 0.6700 & 1 \\
\hline 5 & 0.6900 & 0.6900 & 1 & 5 & 0.6700 & 0.6700 & 1 \\
\hline 5 & 0.6900 & 0.6900 & 1 & 5 & 0.6800 & 0.6800 & 1 \\
\hline 6 & 0.6800 & 0.6800 & 1 & 6 & 0.6800 & 0.6800 & 1 \\
\hline 6 & 0.6800 & 0.6800 & 1 & 6 & 0.6800 & 0.6800 & 1 \\
\hline 6 & 0.6700 & 0.6800 & 1 & 6 & 0.6800 & 0.6800 & 1 \\
\hline 6 & 0.6800 & 0.6900 & 1 & 6 & 0.6800 & 0.6800 & 1 \\
\hline 6 & 0.6900 & 0.6900 & 1 & 6 & 0.6800 & 0.6800 & 1 \\
\hline 6 & 0.6900 & 0.6800 & 1 & 6 & 0.6800 & 0.6800 & 1 \\
\hline 7 & 0.7000 & 0.7000 & 1 & 7 & 0.6800 & 0.6800 & 1 \\
\hline 7 & 0.7000 & 0.7000 & 1 & 7 & 0.6700 & 0.6700 & 1 \\
\hline 7 & 0.7000 & 0.7000 & 1 & 7 & 0.6800 & 0.6800 & 1 \\
\hline 7 & 0.7000 & 0.7000 & 1 & 7 & 0.6800 & 0.6800 & 1 \\
\hline 7 & 0.7000 & 0.7000 & 1 & 7 & 0.6700 & 0.6700 & 1 \\
\hline 7 & 0.7000 & 0.7000 & 1 & 7 & 0.6800 & 0.6800 & 1 \\
\hline 8 & 0.7000 & 0.6980 & 1 & 8 & 0.6800 & 0.6800 & 1 \\
\hline 8 & 0.6980 & 0.7000 & 1 & 8 & 0.6700 & 0.6800 & 1 \\
\hline 8 & 0.7000 & 0.7000 & 1 & 8 & 0.6700 & 0.6700 & 1 \\
\hline
\end{tabular}




\begin{tabular}{|c|c|c|c|c|c|c|}
\hline 8 & 0.6948 & 0.6968 & 1 & 8 & 0.6800 & 0.6800 \\
\hline 8 & 0.6997 & 0.6938 & 1 & 8 & 0.6800 & 0.6800 \\
\hline 8 & 0.6987 & 0.6968 & 1 & 8 & 0.6800 & 0.6800 \\
\hline 9 & 0.6720 & 0.6700 & 1 & 9 & 0.7000 & 0.7000 \\
\hline 9 & 0.6760 & 0.6720 & 1 & 9 & 0.6900 & 0.6900 \\
\hline 9 & 0.6800 & 0.6770 & 1 & 9 & 0.6900 & 0.6900 \\
\hline 9 & 0.6760 & 0.6720 & 1 & 9 & 0.6800 & 0.6800 \\
\hline 9 & 0.6790 & 0.6780 & 1 & 9 & 0.6900 & 0.6900 \\
\hline 9 & 0.6770 & 0.6840 & 1 & 9 & 0.6900 & 0.6900 \\
\hline 10 & 0.7000 & 0.7000 & 1 & 10 & 0.7000 & 0.6900 \\
\hline 10 & 0.7000 & 0.7000 & 1 & 10 & 0.6900 & 0.6900 \\
\hline 10 & 0.7000 & 0.7000 & 1 & 10 & 0.6900 & 0.6900 \\
\hline 10 & 0.7100 & 0.7100 & 1 & 10 & 0.6800 & 0.6800 \\
\hline 10 & 0.7100 & 0.7100 & 1 & 10 & 0.6900 & 0.6900 \\
\hline 10 & 0.7100 & 0.7100 & 1 & 10 & 0.6900 & 0.6900 \\
\hline 11 & 0.6690 & 0.6680 & 1 & 11 & 0.6300 & 0.6500 \\
\hline 11 & 0.6750 & 0.6780 & 1 & 11 & 0.3400 & 0.6400 \\
\hline 11 & 0.6810 & 0.6870 & 1 & 11 & 0.6500 & 0.6500 \\
\hline 11 & 0.6650 & 0.6710 & 1 & 11 & 0.6400 & 0.6300 \\
\hline 11 & 0.6770 & 0.6730 & 1 & 11 & 0.6400 & 0.6500 \\
\hline 11 & 0.6780 & 0.6770 & 1 & 11 & 0.6300 & 0.6500 \\
\hline 12 & 0.6890 & 0.6890 & 1 & 12 & 0.6800 & 0.6700 \\
\hline 12 & 0.6870 & 0.6810 & 1 & 12 & 0.6900 & 0.6900 \\
\hline 12 & 0.6810 & 0.6970 & 1 & 12 & 0.6800 & 0.6800 \\
\hline 12 & 0.6760 & 0.6750 & 1 & 12 & 0.6800 & 0.6800 \\
\hline 12 & 0.6880 & 0.6880 & 1 & 12 & 0.6800 & 0.6800 \\
\hline 12 & 0.6750 & 0.6940 & 1 & 12 & 0.6800 & 0.6800 \\
\hline 13 & 0.6900 & 0.6900 & 1 & 13 & 0.6900 & 0.6800 \\
\hline 13 & 0.6900 & 0.6900 & 1 & 13 & 0.6800 & 0.6800 \\
\hline 13 & 0.6900 & 0.6900 & 1 & 13 & 0.6800 & 0.7000 \\
\hline 13 & 0.6800 & 0.6800 & 1 & 13 & 0.6800 & 0.6800 \\
\hline 13 & 0.6800 & 0.6800 & 1 & 13 & 0.6800 & 0.6800 \\
\hline 13 & 0.6800 & 0.6800 & 1 & 13 & 0.6900 & 0.6800 \\
\hline 14 & 0.6836 & 0.6833 & 1 & 14 & 0.6800 & 0.6900 \\
\hline 14 & 0.6806 & 0.6823 & 1 & 14 & 0.6900 & 0.6900 \\
\hline 14 & 0.6821 & 0.6819 & 1 & 14 & 0.6900 & 0.6900 \\
\hline 14 & 0.6832 & 0.6826 & 1 & 14 & 0.6800 & 0.6800 \\
\hline 14 & 0.6855 & 0.6851 & 1 & 14 & 0.6800 & 0.6800 \\
\hline 14 & 0.6853 & 0.6859 & 1 & 14 & 0.6800 & 0.6900 \\
\hline 15 & 0.6920 & 0.6930 & 1 & 15 & 0.6800 & 0.6800 \\
\hline 15 & 0.6880 & 0.6870 & 1 & 15 & 0.6800 & 0.6800 \\
\hline 15 & 0.6880 & 0.6890 & 1 & 15 & 0.6800 & 0.6800 \\
\hline 15 & 0.6900 & 0.6910 & 1 & 15 & 0.6800 & 0.6800 \\
\hline 15 & 0.6900 & 0.6890 & 1 & 15 & 0.6800 & 0.6800 \\
\hline 15 & 0.6900 & 0.6910 & 1 & 15 & 0.6800 & 0.6800 \\
\hline 16 & 0.6900 & 0.6900 & 1 & 16 & 0.6700 & 0.6700 \\
\hline 16 & 0.6900 & 0.6800 & 1 & 16 & 0.6800 & 0.6800 \\
\hline 16 & 0.6800 & 0.6900 & 1 & 16 & 0.6700 & 0.6800 \\
\hline 16 & 0.6900 & 0.6900 & 1 & 16 & 0.6800 & 0.6800 \\
\hline
\end{tabular}




\begin{tabular}{|c|c|c|c|c|c|c|}
\hline 16 & 0.6900 & 0.6800 & 1 & 16 & 0.6700 & 0.6700 \\
\hline 16 & 0.6900 & 0.6900 & 1 & 16 & 0.6700 & 0.6700 \\
\hline 17 & 0.6900 & 0.6820 & 1 & 17 & 0.6740 & 0.6733 \\
\hline 17 & 0.6860 & 0.6900 & 1 & 17 & 0.6847 & 0.6848 \\
\hline 17 & 0.6810 & 0.6860 & 1 & 17 & 0.6870 & 0.6876 \\
\hline 17 & 0.6820 & 0.6870 & 1 & 17 & 0.6758 & 0.6751 \\
\hline 17 & 0.6840 & 0.6780 & 1 & 17 & 0.6850 & 0.6856 \\
\hline 17 & 0.6830 & 0.6840 & 1 & 17 & 0.6878 & 0.6876 \\
\hline 18 & 0.6800 & 0.6800 & 1 & 18 & 0.7100 & 0.7100 \\
\hline 18 & 0.6700 & 0.6700 & 1 & 18 & 0.7100 & 0.7100 \\
\hline 18 & 0.6700 & 0.6700 & 1 & 18 & 0.7100 & 0.7100 \\
\hline 18 & 0.6700 & 0.6700 & 1 & 18 & 0.7100 & 0.7100 \\
\hline 18 & 0.6800 & 0.6700 & 1 & 18 & 0.7100 & 0.7100 \\
\hline 18 & 0.6700 & 0.6700 & 1 & 18 & 0.7000 & 0.7000 \\
\hline 19 & 0.6800 & 0.6900 & 1 & 19 & 0.6577 & 0.6545 \\
\hline 19 & 0.6800 & 0.6800 & 1 & 19 & 0.6560 & 0.6552 \\
\hline 19 & 0.6800 & 0.6900 & 1 & 19 & 0.6570 & 0.6557 \\
\hline 19 & 0.6800 & 0.6800 & 1 & 19 & 0.6557 & 0.6556 \\
\hline 19 & 0.6900 & 0.6900 & 1 & 19 & 0.6548 & 0.6558 \\
\hline 19 & 0.6900 & 0.6900 & 1 & 19 & 0.6562 & 0.6545 \\
\hline 1 & 0.3800 & 0.3800 & 2 & 20 & 0.6780 & 0.6770 \\
\hline 1 & 0.3800 & 0.3800 & 2 & 20 & 0.6820 & 0.6820 \\
\hline 1 & 0.3900 & 0.3800 & 2 & 20 & 0.6850 & 0.6840 \\
\hline 1 & 0.3800 & 0.3800 & 2 & 20 & 0.6870 & 0.6870 \\
\hline 1 & 0.3800 & 0.3800 & 2 & 20 & 0.6810 & 0.6830 \\
\hline 1 & 0.3800 & 0.3800 & 2 & 20 & 0.6740 & 0.6750 \\
\hline 2 & 0.3800 & 0.3800 & 2 & 21 & 0.6900 & 0.6900 \\
\hline 2 & 0.3800 & 0.3800 & 2 & 21 & 0.6980 & 0.6980 \\
\hline 2 & 0.3800 & 0.3800 & 2 & 21 & 0.7120 & 0.7110 \\
\hline 2 & 0.3800 & 0.3800 & 2 & 21 & 0.6920 & 0.6930 \\
\hline 2 & 0.3800 & 0.3800 & 2 & 21 & 0.6950 & 0.6960 \\
\hline 2 & 0.3800 & 0.3800 & 2 & 21 & 0.6960 & 0.6970 \\
\hline 3 & 0.3870 & 0.3900 & 2 & 22 & 0.7000 & 0.7000 \\
\hline 3 & 0.3930 & 0.3880 & 2 & 22 & 0.6900 & 0.6900 \\
\hline 3 & 0.3880 & 0.3860 & 2 & 22 & 0.6900 & 0.6900 \\
\hline 3 & 0.3880 & 0.3900 & 2 & 22 & 0.6800 & 0.6800 \\
\hline 3 & 0.3880 & 0.3850 & 2 & 22 & 0.6900 & 0.6900 \\
\hline 3 & 0.3900 & 0.3900 & 2 & 22 & 0.6800 & 0.6900 \\
\hline 4 & 0.3900 & 0.3800 & 2 & 23 & 0.6720 & 0.6720 \\
\hline 4 & 0.3800 & 0.3900 & 2 & 23 & 0.6710 & 0.6710 \\
\hline 4 & 0.3800 & 0.3900 & 2 & 23 & 0.6770 & 0.6780 \\
\hline 4 & 0.3900 & 0.3900 & 2 & 23 & 0.6750 & 0.6750 \\
\hline 4 & 0.3900 & 0.3900 & 2 & 23 & 0.6740 & 0.6760 \\
\hline 4 & 0.3800 & 0.3900 & 2 & 23 & 0.6740 & 0.6740 \\
\hline 5 & 0.3900 & 0.3900 & 2 & 24 & 0.6800 & 0.6800 \\
\hline 5 & 0.3900 & 0.3900 & 2 & 24 & 0.6700 & 0.6700 \\
\hline 5 & 0.4000 & 0.3900 & 2 & 24 & 0.6700 & 0.6700 \\
\hline 5 & 0.3900 & 0.3900 & 2 & 24 & 0.6800 & 0.6800 \\
\hline 5 & 0.3900 & 0.3900 & 2 & 24 & 0.6700 & 0.6800 \\
\hline
\end{tabular}




\begin{tabular}{|c|c|c|c|c|c|c|}
\hline 5 & 0.3900 & 0.3900 & 2 & 24 & 0.6800 & 0.6800 \\
\hline 6 & 0.3800 & 0.3800 & 2 & 25 & 0.6940 & 0.6936 \\
\hline 6 & 0.3800 & 0.3800 & 2 & 25 & 0.6897 & 0.6898 \\
\hline 6 & 0.3800 & 0.3800 & 2 & 25 & 0.6887 & 0.6879 \\
\hline 6 & 0.3800 & 0.3800 & 2 & 25 & 0.6910 & 0.6898 \\
\hline 6 & 0.3800 & 0.3800 & 2 & 25 & 0.6868 & 0.6869 \\
\hline 6 & 0.3800 & 0.3800 & 2 & 25 & 0.6906 & 0.6896 \\
\hline 7 & 0.4100 & 0.4100 & 2 & 26 & 0.6900 & 0.6800 \\
\hline 7 & 0.4100 & 0.4100 & 2 & 26 & 0.6800 & 0.6800 \\
\hline 7 & 0.4100 & 0.4100 & 2 & 26 & 0.6900 & 0.6900 \\
\hline 7 & 0.4100 & 0.4100 & 2 & 26 & 0.6900 & 0.6900 \\
\hline 7 & 0.4100 & 0.4100 & 2 & 26 & 0.6900 & 0.6800 \\
\hline 7 & 0.4100 & 0.4100 & 2 & 26 & 0.6900 & 0.6800 \\
\hline 8 & 0.3919 & 0.3919 & 2 & 1 & 0.3350 & 0.3350 \\
\hline 8 & 0.3929 & 0.3919 & 2 & 1 & 0.3390 & 0.3400 \\
\hline 8 & 0.3919 & 0.3939 & 2 & 1 & 0.3340 & 0.3360 \\
\hline 8 & 0.3951 & 0.3932 & 2 & 1 & 0.3410 & 0.3400 \\
\hline 8 & 0.3942 & 0.3932 & 2 & 1 & 0.3400 & 0.3390 \\
\hline 8 & 0.3932 & 0.3941 & 2 & 1 & 0.3330 & 0.3340 \\
\hline 9 & 0.3810 & 0.3790 & 2 & 2 & 0.3820 & 0.3820 \\
\hline 9 & 0.3800 & 0.3810 & 2 & 2 & 0.3760 & 0.3760 \\
\hline 9 & 0.3830 & 0.3790 & 2 & 2 & 0.3770 & 0.3770 \\
\hline 9 & 0.3780 & 0.3790 & 2 & 2 & 0.3820 & 0.3810 \\
\hline 9 & 0.3810 & 0.3790 & 2 & 2 & 0.3760 & 0.3760 \\
\hline 9 & 0.3820 & 0.3830 & 2 & 2 & 0.3760 & 0.3770 \\
\hline 10 & 0.3900 & 0.3900 & 2 & 3 & 0.3800 & 0.3800 \\
\hline 10 & 0.3900 & 0.3900 & 2 & 3 & 0.3800 & 0.3800 \\
\hline 10 & 0.3900 & 0.3900 & 2 & 3 & 0.3800 & 0.3800 \\
\hline 10 & 0.3800 & 0.3800 & 2 & 3 & 0.3800 & 0.3800 \\
\hline 10 & 0.3800 & 0.3800 & 2 & 3 & 0.3800 & 0.3800 \\
\hline 10 & 0.3800 & 0.3800 & 2 & 3 & 0.3800 & 0.3700 \\
\hline 11 & 0.3810 & 0.3730 & 2 & 4 & 0.3930 & 0.3940 \\
\hline 11 & 0.3790 & 0.3810 & 2 & 4 & 0.3920 & 0.3920 \\
\hline 11 & 0.3790 & 0.3900 & 2 & 4 & 0.3930 & 0.3930 \\
\hline 11 & 0.3820 & 0.3790 & 2 & 4 & 0.3950 & 0.3950 \\
\hline 11 & 0.3860 & 0.3840 & 2 & 4 & 0.3950 & 0.3950 \\
\hline 11 & 0.3900 & 0.3800 & 2 & 4 & 0.3930 & 0.3930 \\
\hline 12 & 0.3710 & 0.3720 & 2 & 5 & 0.3700 & 0.3700 \\
\hline 12 & 0.3720 & 0.3740 & 2 & 5 & 0.3700 & 0.3600 \\
\hline 12 & 0.3730 & 0.3860 & 2 & 5 & 0.3600 & 0.3600 \\
\hline 12 & 0.3860 & 0.3860 & 2 & 5 & 0.3700 & 0.3800 \\
\hline 12 & 0.3750 & 0.3740 & 2 & 5 & 0.3700 & 0.3600 \\
\hline 12 & 0.3840 & 0.3840 & 2 & 5 & 0.3600 & 0.3700 \\
\hline 13 & 0.3900 & 0.3900 & 2 & 6 & 0.3800 & 0.3800 \\
\hline 13 & 0.3800 & 0.3800 & 2 & 6 & 0.3800 & 0.3800 \\
\hline 13 & 0.3800 & 0.3800 & 2 & 6 & 0.3800 & 0.3800 \\
\hline 13 & 0.3800 & 0.3700 & 2 & 6 & 0.3800 & 0.3800 \\
\hline 13 & 0.3800 & 0.3800 & 2 & 6 & 0.3800 & 0.3800 \\
\hline 13 & 0.3800 & 0.3800 & 2 & 6 & 0.3800 & 0.3800 \\
\hline
\end{tabular}




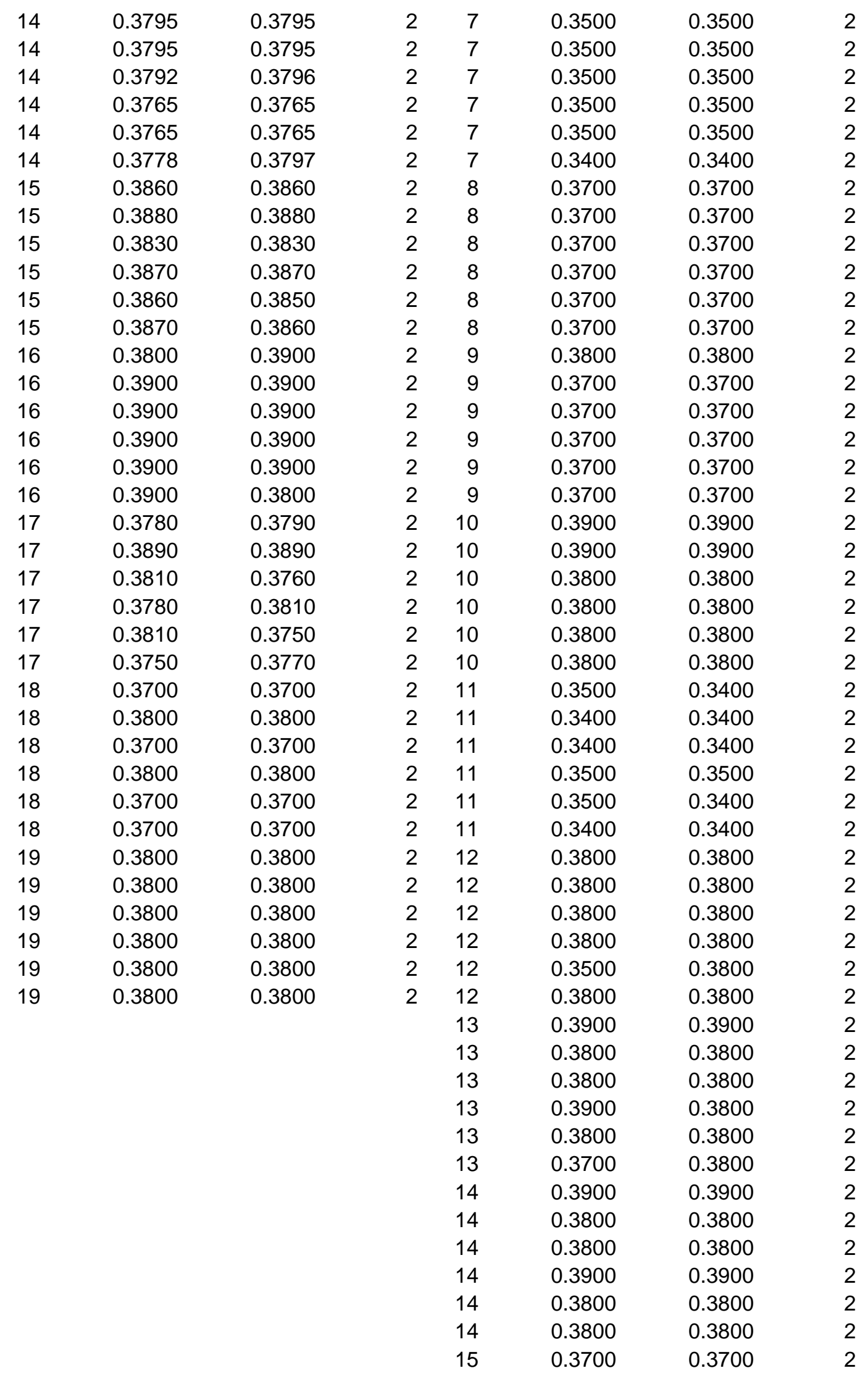




$\begin{array}{llll}15 & 0.3700 & 0.3700 & 2 \\ 15 & 0.3700 & 0.3700 & 2 \\ 15 & 0.3700 & 0.3700 & 2 \\ 15 & 0.3700 & 0.3700 & 2 \\ 15 & 0.3700 & 0.3700 & 2 \\ 16 & 0.3900 & 0.3900 & 2 \\ 16 & 0.3900 & 0.3800 & 2 \\ 16 & 0.3900 & 0.3900 & 2 \\ 16 & 0.3900 & 0.3900 & 2 \\ 16 & 0.3900 & 0.3900 & 2 \\ 16 & 0.3800 & 0.3800 & 2 \\ 17 & 0.3725 & 0.3728 & 2 \\ 17 & 0.3747 & 0.3736 & 2 \\ 17 & 0.3729 & 0.3702 & 2 \\ 17 & 0.3718 & 0.3735 & 2 \\ 17 & 0.3750 & 0.3759 & 2 \\ 17 & 0.3754 & 0.3751 & 2 \\ 18 & 0.3900 & 0.3900 & 2 \\ 18 & 0.3900 & 0.3900 & 2 \\ 18 & 0.3900 & 0.3900 & 2 \\ 18 & 0.4000 & 0.4000 & 2 \\ 18 & 0.4000 & 0.4000 & 2 \\ 18 & 0.3900 & 0.3900 & 2 \\ 19 & 0.3755 & 0.3753 & 2 \\ 19 & 0.3755 & 0.3753 & 2 \\ 19 & 0.3776 & 0.3773 & 2 \\ 19 & 0.3762 & 0.3760 & 2 \\ 19 & 0.3762 & 0.3760 & 2 \\ 19 & 0.3756 & 0.3761 & 2 \\ 20 & 0.3840 & 0.3820 & 2 \\ 20 & 0.3830 & 0.3830 & 2 \\ 20 & 0.3570 & 0.3570 & 2 \\ 20 & 0.3600 & 0.3630 & 2 \\ 20 & 0.3830 & 0.3830 & 2 \\ 20 & 0.3850 & 0.3890 & 2 \\ 21 & 0.3930 & 0.3930 & 2 \\ 21 & 0.4040 & 0.4040 & 2 \\ 21 & 0.3900 & 0.3900 & 2 \\ 21 & 0.4010 & 0.4000 & 2 \\ 21 & 0.3940 & 0.3930 & 2 \\ 21 & 0.3910 & 0.3910 & 2 \\ 22 & 0.3900 & 0.3900 & 2 \\ 22 & 0.3900 & 0.3800 & 2 \\ 22 & 0.3800 & 0.3800 & 2 \\ 22 & 0.3900 & 0.3900 & 2 \\ 22 & 0.3900 & 0.3900 & 2 \\ 22 & 0.3900 & 0.3900 & 2 \\ 23 & 0.3810 & 0.3820 & 2 \\ 23 & 0.3850 & 0.3860 & 2\end{array}$




$\begin{array}{llll}23 & 0.3810 & 0.3820 & 2 \\ 23 & 0.3870 & 0.3850 & 2 \\ 23 & 0.3830 & 0.3840 & 2 \\ 23 & 0.3780 & 0.3800 & 2 \\ 24 & 0.3900 & 0.3900 & 2 \\ 24 & 0.3900 & 0.3900 & 2 \\ 24 & 0.3800 & 0.3800 & 2 \\ 24 & 0.3900 & 0.3900 & 2 \\ 24 & 0.3900 & 0.3900 & 2 \\ 24 & 0.3900 & 0.3900 & 2 \\ 25 & 0.3825 & 0.3822 & 2 \\ 25 & 0.3814 & 0.3808 & 2 \\ 25 & 0.3809 & 0.3806 & 2 \\ 25 & 0.3771 & 0.3773 & 2 \\ 25 & 0.3811 & 0.3806 & 2 \\ 25 & 0.3803 & 0.3802 & 2 \\ 26 & 0.3700 & 0.3800 & 2 \\ 26 & 0.3800 & 0.3800 & 2 \\ 26 & 0.3700 & 0.3800 & 2 \\ 26 & 0.3800 & 0.3800 & 2 \\ 26 & 0.3800 & 0.3800 & 2 \\ 26 & 0.3700 & 0.3800 & 2\end{array}$




\begin{tabular}{|c|c|c|c|c|c|c|c|}
\hline \multicolumn{4}{|c|}{ TiO2: Glass Preparation } & \multicolumn{4}{|c|}{ TiO2: Powder Preparation } \\
\hline Lab & Duplicate 1 & Duplicate 2 & Cement & Lab & Duplicate 1 & Duplicate 2 & Cement \\
\hline 1 & 0.3200 & 0.3200 & 1 & 1 & 0.2900 & 0.2910 & 1 \\
\hline 1 & 0.3200 & 0.3200 & 1 & 1 & 0.2910 & 0.2940 & 1 \\
\hline 1 & 0.3200 & 0.3200 & 1 & 1 & 0.2930 & 0.2870 & 1 \\
\hline 1 & 0.3200 & 0.3200 & 1 & 1 & 0.2900 & 0.2910 & 1 \\
\hline 1 & 0.3200 & 0.3200 & 1 & 1 & 0.2910 & 0.2890 & 1 \\
\hline 1 & 0.3200 & 0.3200 & 1 & 1 & 0.2890 & 0.2930 & 1 \\
\hline 2 & 0.3120 & 0.3060 & 1 & 2 & 0.3030 & 0.3020 & 1 \\
\hline 2 & 0.3080 & 0.3080 & 1 & 2 & 0.3050 & 0.3030 & 1 \\
\hline 2 & 0.3070 & 0.3200 & 1 & 2 & 0.3040 & 0.3030 & 1 \\
\hline 2 & 0.3110 & 0.3070 & 1 & 2 & 0.3010 & 0.3030 & 1 \\
\hline 2 & 0.3160 & 0.3150 & 1 & 2 & 0.3020 & 0.3050 & 1 \\
\hline 2 & 0.3040 & 0.3020 & 1 & 2 & 0.3000 & 0.3020 & 1 \\
\hline 3 & 0.3100 & 0.3100 & 1 & 3 & 0.2600 & 0.2500 & 1 \\
\hline 3 & 0.3100 & 0.3100 & 1 & 3 & 0.2600 & 0.2700 & 1 \\
\hline 3 & 0.3100 & 0.3100 & 1 & 3 & 0.2400 & 0.2600 & 1 \\
\hline 3 & 0.3000 & 0.3100 & 1 & 3 & 0.2600 & 0.2800 & 1 \\
\hline 3 & 0.3100 & 0.3000 & 1 & 3 & 0.2800 & 0.2800 & 1 \\
\hline 3 & 0.3100 & 0.3100 & 1 & 3 & 0.2600 & 0.2600 & 1 \\
\hline 4 & 0.3090 & 0.3060 & 1 & 4 & 0.3000 & 0.3000 & 1 \\
\hline 4 & 0.3100 & 0.3080 & 1 & 4 & 0.3000 & 0.3000 & 1 \\
\hline 4 & 0.3050 & 0.3070 & 1 & 4 & 0.3000 & 0.3000 & 1 \\
\hline 4 & 0.3090 & 0.3070 & 1 & 4 & 0.3000 & 0.3000 & 1 \\
\hline 4 & 0.3090 & 0.3080 & 1 & 4 & 0.3000 & 0.3000 & 1 \\
\hline 4 & 0.3080 & 0.3060 & 1 & 4 & 0.3000 & 0.3000 & 1 \\
\hline 5 & 0.3200 & 0.3200 & 1 & 5 & 0.3160 & 0.3140 & 1 \\
\hline 5 & 0.3200 & 0.3200 & 1 & 5 & 0.3110 & 0.3130 & 1 \\
\hline 5 & 0.3200 & 0.3200 & 1 & 5 & 0.3150 & 0.3110 & 1 \\
\hline 5 & 0.3200 & 0.3200 & 1 & 5 & 0.3110 & 0.3110 & 1 \\
\hline 5 & 0.3200 & 0.3300 & 1 & 5 & 0.3130 & 0.3130 & 1 \\
\hline 5 & 0.3200 & 0.3200 & 1 & 5 & 0.3100 & 0.3080 & 1 \\
\hline 6 & 0.3130 & 0.3020 & 1 & 6 & 0.3020 & 0.3100 & 1 \\
\hline 6 & 0.2980 & 0.3080 & 1 & 6 & 0.3000 & 0.3030 & 1 \\
\hline 6 & 0.3080 & 0.3040 & 1 & 6 & 0.2980 & 0.3030 & 1 \\
\hline 6 & 0.2960 & 0.3090 & 1 & 6 & 0.3000 & 0.3050 & 1 \\
\hline 6 & 0.3020 & 0.3060 & 1 & 6 & 0.3070 & 0.3040 & 1 \\
\hline 6 & 0.2970 & 0.2910 & 1 & 6 & 0.3060 & 0.3070 & 1 \\
\hline 7 & 0.3170 & 0.3170 & 1 & 7 & 0.3000 & 0.3000 & 1 \\
\hline 7 & 0.3210 & 0.3230 & 1 & 7 & 0.3000 & 0.3000 & 1 \\
\hline 7 & 0.3220 & 0.3120 & 1 & 7 & 0.3000 & 0.3000 & 1 \\
\hline 7 & 0.3150 & 0.3250 & 1 & 7 & 0.3000 & 0.3000 & 1 \\
\hline 7 & 0.3160 & 0.3160 & 1 & 7 & 0.3000 & 0.3000 & 1 \\
\hline 7 & 0.3200 & 0.3210 & 1 & 7 & 0.3000 & 0.3000 & 1 \\
\hline 8 & 0.3000 & 0.3000 & 1 & 8 & 0.2880 & 0.2910 & 1 \\
\hline 8 & 0.3000 & 0.3000 & 1 & 8 & 0.2860 & 0.2900 & 1 \\
\hline 8 & 0.3100 & 0.3000 & 1 & 8 & 0.2950 & 0.2840 & 1 \\
\hline 8 & 0.3100 & 0.3000 & 1 & 8 & 0.2830 & 0.2910 & 1 \\
\hline
\end{tabular}




\begin{tabular}{|c|c|c|c|c|c|c|}
\hline 8 & 0.3100 & 0.3000 & 1 & 8 & 0.2850 & 0.2890 \\
\hline 8 & 0.3000 & 0.3000 & 1 & 8 & 0.2870 & 0.2910 \\
\hline 9 & 0.3128 & 0.3145 & 1 & 9 & 0.3200 & 0.3100 \\
\hline 9 & 0.3108 & 0.3111 & 1 & 9 & 0.3100 & 0.3200 \\
\hline 9 & 0.3116 & 0.3123 & 1 & 9 & 0.3200 & 0.3100 \\
\hline 9 & 0.3111 & 0.3106 & 1 & 9 & 0.3200 & 0.3100 \\
\hline 9 & 0.3134 & 0.3122 & 1 & 9 & 0.3200 & 0.3200 \\
\hline 9 & 0.3125 & 0.3112 & 1 & 9 & 0.3200 & 0.3100 \\
\hline 10 & 0.3200 & 0.3100 & 1 & 10 & 0.2900 & 0.3000 \\
\hline 10 & 0.3100 & 0.3200 & 1 & 10 & 0.2900 & 0.3000 \\
\hline 10 & 0.3200 & 0.3100 & 1 & 10 & 0.2900 & 0.2900 \\
\hline 10 & 0.3100 & 0.3100 & 1 & 10 & 0.3000 & 0.3000 \\
\hline 10 & 0.3200 & 0.3200 & 1 & 10 & 0.2900 & 0.2900 \\
\hline 10 & 0.3200 & 0.3100 & 1 & 10 & 0.3000 & 0.2900 \\
\hline 11 & 0.3060 & 0.3040 & 1 & 11 & 0.3063 & 0.3062 \\
\hline 11 & 0.3050 & 0.3060 & 1 & 11 & 0.3067 & 0.3053 \\
\hline 11 & 0.3030 & 0.3050 & 1 & 11 & 0.3061 & 0.3049 \\
\hline 11 & 0.3050 & 0.3030 & 1 & 11 & 0.3047 & 0.3046 \\
\hline 11 & 0.3080 & 0.3050 & 1 & 11 & 0.3069 & 0.3055 \\
\hline 11 & 0.3060 & 0.3060 & 1 & 11 & 0.3046 & 0.3055 \\
\hline 12 & 0.3100 & 0.3000 & 1 & 12 & 0.3070 & 0.3060 \\
\hline 12 & 0.3000 & 0.3000 & 1 & 12 & 0.3110 & 0.3030 \\
\hline 12 & 0.3000 & 0.3000 & 1 & 12 & 0.3070 & 0.3080 \\
\hline 12 & 0.3100 & 0.3100 & 1 & 12 & 0.3010 & 0.3110 \\
\hline 12 & 0.3100 & 0.3100 & 1 & 12 & 0.3110 & 0.3100 \\
\hline 12 & 0.3100 & 0.3100 & 1 & 12 & 0.3030 & 0.3140 \\
\hline 13 & 0.3160 & 0.3160 & 1 & 13 & 0.3001 & 0.2967 \\
\hline 13 & 0.3200 & 0.3210 & 1 & 13 & 0.2904 & 0.2955 \\
\hline 13 & 0.3170 & 0.3150 & 1 & 13 & 0.2990 & 0.3000 \\
\hline 13 & 0.3100 & 0.3140 & 1 & 13 & 0.2966 & 0.2985 \\
\hline 13 & 0.3160 & 0.3180 & 1 & 13 & 0.2947 & 0.2955 \\
\hline 13 & 0.3170 & 0.3170 & 1 & 13 & 0.2970 & 0.2978 \\
\hline 14 & 0.3000 & 0.3100 & 1 & 14 & 0.3000 & 0.2900 \\
\hline 14 & 0.3000 & 0.3000 & 1 & 14 & 0.3000 & 0.3100 \\
\hline 14 & 0.3000 & 0.3000 & 1 & 14 & 0.3000 & 0.3000 \\
\hline 14 & 0.3100 & 0.3000 & 1 & 14 & 0.3000 & 0.3000 \\
\hline 14 & 0.3000 & 0.3100 & 1 & 14 & 0.3000 & 0.3000 \\
\hline 14 & 0.3100 & 0.3000 & 1 & 14 & 0.2900 & 0.2900 \\
\hline 15 & 0.2970 & 0.2970 & 1 & 15 & 0.3100 & 0.3100 \\
\hline 15 & 0.2960 & 0.3070 & 1 & 15 & 0.3100 & 0.3100 \\
\hline 15 & 0.2980 & 0.3000 & 1 & 15 & 0.3100 & 0.3100 \\
\hline 15 & 0.2980 & 0.2970 & 1 & 15 & 0.3100 & 0.3200 \\
\hline 15 & 0.2990 & 0.3080 & 1 & 15 & 0.3100 & 0.3100 \\
\hline 15 & 0.3020 & 0.3110 & 1 & 15 & 0.3100 & 0.3100 \\
\hline 16 & 0.3000 & 0.3000 & 1 & 16 & 0.3000 & 0.3100 \\
\hline 16 & 0.3000 & 0.3000 & 1 & 16 & 0.3000 & 0.3100 \\
\hline 16 & 0.3000 & 0.3000 & 1 & 16 & 0.3000 & 0.3000 \\
\hline 16 & 0.3000 & 0.3000 & 1 & 16 & 0.3000 & 0.3000 \\
\hline 16 & 0.3100 & 0.3100 & 1 & 16 & 0.3000 & 0.3000 \\
\hline
\end{tabular}




\begin{tabular}{|c|c|c|c|c|c|c|}
\hline 16 & 0.3100 & 0.3100 & 1 & 16 & 0.3000 & 0.3000 \\
\hline 17 & 0.3000 & 0.3100 & 1 & 17 & 0.3100 & 0.3120 \\
\hline 17 & 0.3100 & 0.3100 & 1 & 17 & 0.3090 & 0.3100 \\
\hline 17 & 0.3000 & 0.3100 & 1 & 17 & 0.3140 & 0.3130 \\
\hline 17 & 0.3100 & 0.3100 & 1 & 17 & 0.3140 & 0.3140 \\
\hline 17 & 0.3100 & 0.3100 & 1 & 17 & 0.3100 & 0.3130 \\
\hline 17 & 0.3100 & 0.3000 & 1 & 17 & 0.3120 & 0.3130 \\
\hline 1 & 0.3500 & 0.3500 & 2 & 18 & 0.3000 & 0.3100 \\
\hline 1 & 0.3400 & 0.3400 & 2 & 18 & 0.3100 & 0.3000 \\
\hline 1 & 0.3500 & 0.3400 & 2 & 18 & 0.3100 & 0.3000 \\
\hline 1 & 0.3400 & 0.3400 & 2 & 18 & 0.3000 & 0.3000 \\
\hline 1 & 0.3400 & 0.3500 & 2 & 18 & 0.3000 & 0.3000 \\
\hline 1 & 0.3400 & 0.3400 & 2 & 18 & 0.3000 & 0.3000 \\
\hline 2 & 0.3400 & 0.3350 & 2 & 19 & 0.3100 & 0.3100 \\
\hline 2 & 0.3350 & 0.3310 & 2 & 19 & 0.3100 & 0.3100 \\
\hline 2 & 0.3350 & 0.3270 & 2 & 19 & 0.3100 & 0.3100 \\
\hline 2 & 0.3330 & 0.3370 & 2 & 19 & 0.3100 & 0.3100 \\
\hline 2 & 0.3310 & 0.3420 & 2 & 19 & 0.3100 & 0.3100 \\
\hline 2 & 0.3380 & 0.3380 & 2 & 19 & 0.3100 & 0.3100 \\
\hline 3 & 0.3400 & 0.3300 & 2 & 20 & 0.3020 & 0.2998 \\
\hline 3 & 0.3300 & 0.3400 & 2 & 20 & 0.3028 & 0.3012 \\
\hline 3 & 0.3300 & 0.3400 & 2 & 20 & 0.3063 & 0.3035 \\
\hline 3 & 0.3400 & 0.3300 & 2 & 20 & 0.3008 & 0.3000 \\
\hline 3 & 0.3300 & 0.3300 & 2 & 20 & 0.3019 & 0.3013 \\
\hline 3 & 0.3400 & 0.3300 & 2 & 20 & 0.3042 & 0.3049 \\
\hline 4 & 0.3330 & 0.3330 & 2 & 1 & 0.3190 & 0.3190 \\
\hline 4 & 0.3320 & 0.3300 & 2 & 1 & 0.3230 & 0.3210 \\
\hline 4 & 0.3310 & 0.3310 & 2 & 1 & 0.3200 & 0.3220 \\
\hline 4 & 0.3350 & 0.3330 & 2 & 1 & 0.3170 & 0.3150 \\
\hline 4 & 0.3340 & 0.3320 & 2 & 1 & 0.3220 & 0.3230 \\
\hline 4 & 0.3330 & 0.3350 & 2 & 1 & 0.3250 & 0.3210 \\
\hline 5 & 0.3400 & 0.3400 & 2 & 2 & 0.3340 & 0.3360 \\
\hline 5 & 0.3400 & 0.3500 & 2 & 2 & 0.3360 & 0.3340 \\
\hline 5 & 0.3400 & 0.3400 & 2 & 2 & 0.3350 & 0.3340 \\
\hline 5 & 0.3500 & 0.3400 & 2 & 2 & 0.3340 & 0.3340 \\
\hline 5 & 0.3500 & 0.3500 & 2 & 2 & 0.3350 & 0.3290 \\
\hline 5 & 0.3400 & 0.3400 & 2 & 2 & 0.3340 & 0.3320 \\
\hline 6 & 0.3260 & 0.3280 & 2 & 3 & 0.3000 & 0.2900 \\
\hline 6 & 0.3290 & 0.3210 & 2 & 3 & 0.2900 & 0.3000 \\
\hline 6 & 0.3290 & 0.3230 & 2 & 3 & 0.3300 & 0.2800 \\
\hline 6 & 0.3210 & 0.3210 & 2 & 3 & 0.2900 & 0.3100 \\
\hline 6 & 0.3310 & 0.3310 & 2 & 3 & 0.3000 & 0.2900 \\
\hline 6 & 0.3310 & 0.3340 & 2 & 3 & 0.3000 & 0.3000 \\
\hline 7 & 0.3360 & 0.3490 & 2 & 4 & 0.3300 & 0.3300 \\
\hline 7 & 0.3460 & 0.3370 & 2 & 4 & 0.3300 & 0.3300 \\
\hline 7 & 0.3370 & 0.3420 & 2 & 4 & 0.3300 & 0.3300 \\
\hline 7 & 0.3410 & 0.3410 & 2 & 4 & 0.3300 & 0.3300 \\
\hline 7 & 0.3430 & 0.3420 & 2 & 4 & 0.3300 & 0.3300 \\
\hline 7 & 0.3450 & 0.3450 & 2 & 4 & 0.3300 & 0.3300 \\
\hline
\end{tabular}




\begin{tabular}{|c|c|c|c|c|c|c|}
\hline 8 & 0.3300 & 0.3200 & 2 & 5 & 0.3410 & 0.3390 \\
\hline 8 & 0.3200 & 0.3300 & 2 & 5 & 0.3400 & 0.3380 \\
\hline 8 & 0.3300 & 0.3300 & 2 & 5 & 0.3440 & 0.3460 \\
\hline 8 & 0.3300 & 0.3300 & 2 & 5 & 0.3450 & 0.3480 \\
\hline 8 & 0.3300 & 0.3300 & 2 & 5 & 0.3430 & 0.3420 \\
\hline 8 & 0.3200 & 0.3300 & 2 & 5 & 0.3370 & 0.3350 \\
\hline 9 & 0.3415 & 0.3428 & 2 & 6 & 0.3350 & 0.3400 \\
\hline 9 & 0.3415 & 0.3428 & 2 & 6 & 0.3330 & 0.3350 \\
\hline 9 & 0.3424 & 0.3440 & 2 & 6 & 0.3420 & 0.3380 \\
\hline 9 & 0.3424 & 0.3426 & 2 & 6 & 0.3360 & 0.3330 \\
\hline 9 & 0.3424 & 0.3426 & 2 & 6 & 0.3420 & 0.3450 \\
\hline 9 & 0.3429 & 0.3429 & 2 & 6 & 0.3340 & 0.3360 \\
\hline 10 & 0.3400 & 0.3400 & 2 & 7 & 0.3300 & 0.3300 \\
\hline 10 & 0.3400 & 0.3400 & 2 & 7 & 0.3300 & 0.3200 \\
\hline 10 & 0.3400 & 0.3400 & 2 & 7 & 0.3300 & 0.3300 \\
\hline 10 & 0.3400 & 0.3400 & 2 & 7 & 0.3300 & 0.3300 \\
\hline 10 & 0.3400 & 0.3400 & 2 & 7 & 0.3300 & 0.3300 \\
\hline 10 & 0.3400 & 0.3400 & 2 & 7 & 0.3400 & 0.3300 \\
\hline 11 & 0.3320 & 0.3280 & 2 & 8 & 0.3340 & 0.3280 \\
\hline 11 & 0.3290 & 0.3290 & 2 & 8 & 0.3330 & 0.3330 \\
\hline 11 & 0.3310 & 0.3300 & 2 & 8 & 0.3310 & 0.3290 \\
\hline 11 & 0.3310 & 0.3310 & 2 & 8 & 0.3290 & 0.3290 \\
\hline 11 & 0.3310 & 0.3330 & 2 & 8 & 0.3310 & 0.3350 \\
\hline 11 & 0.3330 & 0.3320 & 2 & 8 & 0.3320 & 0.3290 \\
\hline 12 & 0.3300 & 0.3300 & 2 & 9 & 0.3600 & 0.3600 \\
\hline 12 & 0.3300 & 0.3200 & 2 & 9 & 0.3600 & 0.3600 \\
\hline 12 & 0.3300 & 0.3200 & 2 & 9 & 0.3600 & 0.3600 \\
\hline 12 & 0.3400 & 0.3300 & 2 & 9 & 0.3700 & 0.3600 \\
\hline 12 & 0.3400 & 0.3300 & 2 & 9 & 0.3600 & 0.3600 \\
\hline 12 & 0.3300 & 0.3300 & 2 & 9 & 0.3600 & 0.3600 \\
\hline 13 & 0.3340 & 0.3350 & 2 & 10 & 0.3200 & 0.3200 \\
\hline 13 & 0.3390 & 0.3350 & 2 & 10 & 0.3200 & 0.3300 \\
\hline 13 & 0.3400 & 0.3430 & 2 & 10 & 0.3200 & 0.3200 \\
\hline 13 & 0.3360 & 0.3330 & 2 & 10 & 0.3300 & 0.3300 \\
\hline 13 & 0.3460 & 0.3390 & 2 & 10 & 0.3200 & 0.3200 \\
\hline 13 & 0.3340 & 0.3340 & 2 & 10 & 0.3200 & 0.3300 \\
\hline 14 & 0.3300 & 0.3300 & 2 & 11 & 0.3409 & 0.3409 \\
\hline 14 & 0.3300 & 0.3200 & 2 & 11 & 0.3409 & 0.3409 \\
\hline 14 & 0.3300 & 0.3300 & 2 & 11 & 0.3416 & 0.3422 \\
\hline 14 & 0.3300 & 0.3300 & 2 & 11 & 0.3416 & 0.3412 \\
\hline 14 & 0.3200 & 0.3300 & 2 & 11 & 0.3416 & 0.3412 \\
\hline 14 & 0.3300 & 0.3300 & 2 & 11 & 0.3418 & 0.3417 \\
\hline 15 & 0.3240 & 0.3340 & 2 & 12 & 0.3390 & 0.3390 \\
\hline 15 & 0.3150 & 0.3260 & 2 & 12 & 0.3400 & 0.3410 \\
\hline 15 & 0.3160 & 0.3180 & 2 & 12 & 0.3400 & 0.3390 \\
\hline 15 & 0.3300 & 0.3200 & 2 & 12 & 0.3390 & 0.3440 \\
\hline 15 & 0.3290 & 0.3190 & 2 & 12 & 0.3370 & 0.3420 \\
\hline 15 & 0.3280 & 0.3180 & 2 & 12 & 0.3380 & 0.3380 \\
\hline 16 & 0.3200 & 0.3200 & 2 & 13 & 0.3203 & 0.3223 \\
\hline
\end{tabular}




\begin{tabular}{|c|c|c|c|c|c|}
\hline 0.3200 & 0.3200 & 2 & 13 & 0.3228 & 0.3198 \\
\hline 0.3200 & 0.3200 & 2 & 13 & 0.3245 & 0.3225 \\
\hline 0.3300 & 0.3300 & 2 & 13 & 0.3186 & 0.3204 \\
\hline 0.3300 & 0.3300 & 2 & 13 & 0.3234 & 0.3220 \\
\hline 0.3300 & 0.3300 & 2 & 13 & 0.3236 & 0.3268 \\
\hline 0.3300 & 0.3300 & 2 & 14 & 0.3300 & 0.3300 \\
\hline 0.3300 & 0.3300 & 2 & 14 & 0.3200 & 0.3300 \\
\hline 0.3300 & 0.3300 & 2 & 14 & 0.3300 & 0.3200 \\
\hline 0.3200 & 0.3300 & 2 & 14 & 0.3300 & 0.3300 \\
\hline 0.3400 & 0.3200 & 2 & 14 & 0.3200 & 0.3200 \\
\hline \multirow[t]{37}{*}{0.3300} & 0.3300 & 2 & 14 & 0.3200 & 0.3200 \\
\hline & & & 15 & 0.3300 & 0.3400 \\
\hline & & & 15 & 0.3400 & 0.3400 \\
\hline & & & 15 & 0.3400 & 0.3400 \\
\hline & & & 15 & 0.3400 & 0.3400 \\
\hline & & & 15 & 0.3400 & 0.3400 \\
\hline & & & 15 & 0.3400 & 0.3400 \\
\hline & & & 16 & 0.3300 & 0.3300 \\
\hline & & & 16 & 0.3300 & 0.3300 \\
\hline & & & 16 & 0.3300 & 0.3400 \\
\hline & & & 16 & 0.3300 & 0.3300 \\
\hline & & & 16 & 0.3400 & 0.3300 \\
\hline & & & 16 & 0.3300 & 0.3300 \\
\hline & & & 17 & 0.3410 & 0.3430 \\
\hline & & & 17 & 0.3400 & 0.3450 \\
\hline & & & 17 & 0.3390 & 0.3430 \\
\hline & & & 17 & 0.3390 & 0.3440 \\
\hline & & & 17 & 0.3410 & 0.3430 \\
\hline & & & 17 & 0.3440 & 0.3470 \\
\hline & & & 18 & 0.3300 & 0.3300 \\
\hline & & & 18 & 0.3300 & 0.3300 \\
\hline & & & 18 & 0.3300 & 0.3300 \\
\hline & & & 18 & 0.3300 & 0.3300 \\
\hline & & & 18 & 0.3300 & 0.3300 \\
\hline & & & 18 & 0.3300 & 0.3300 \\
\hline & & & 19 & 0.3300 & 0.3300 \\
\hline & & & 19 & 0.3300 & 0.3300 \\
\hline & & & 19 & 0.3300 & 0.3300 \\
\hline & & & 19 & 0.3300 & 0.3200 \\
\hline & & & 19 & 0.3300 & 0.3300 \\
\hline & & & 19 & 0.3300 & 0.3300 \\
\hline & & & 20 & 0.3334 & 0.3345 \\
\hline & & & 20 & 0.3320 & 0.3318 \\
\hline & & & 20 & 0.3293 & 0.3279 \\
\hline & & & 20 & 0.3285 & 0.3310 \\
\hline & & & 20 & 0.3332 & 0.3312 \\
\hline & & & 20 & 0.3334 & 0.3317 \\
\hline
\end{tabular}




\begin{tabular}{|c|c|c|c|c|c|c|c|}
\hline \multicolumn{4}{|c|}{ P205: Glass Preparation } & \multicolumn{4}{|c|}{ P205: Powder Preparation } \\
\hline Lab & Duplicate 1 & Duplicate 2 & Cement & Lab & Duplicate 1 & Duplicate 2 & Cement \\
\hline 1 & 0.2070 & 0.2130 & 1 & 1 & 0.2200 & 0.2200 & 1 \\
\hline 1 & 0.2000 & 0.2090 & 1 & 1 & 0.2200 & 0.2200 & 1 \\
\hline 1 & 0.2200 & 0.2110 & 1 & 1 & 0.2200 & 0.2200 & 1 \\
\hline 1 & 0.2100 & 0.2160 & 1 & 1 & 0.2200 & 0.2200 & 1 \\
\hline 1 & 0.1990 & 0.2110 & 1 & 1 & 0.2200 & 0.2200 & 1 \\
\hline 1 & 0.1990 & 0.2120 & 1 & 1 & 0.2200 & 0.2200 & 1 \\
\hline 2 & 0.2114 & 0.2114 & 1 & 2 & 0.2740 & 0.2760 & 1 \\
\hline 2 & 0.2114 & 0.2104 & 1 & 2 & 0.2750 & 0.2790 & 1 \\
\hline 2 & 0.2114 & 0.2104 & 1 & 2 & 0.2740 & 0.2770 & 1 \\
\hline 2 & 0.2096 & 0.2116 & 1 & 2 & 0.2750 & 0.2790 & 1 \\
\hline 2 & 0.2106 & 0.2116 & 1 & 2 & 0.2770 & 0.0275 & 1 \\
\hline 2 & 0.2106 & 0.2106 & 1 & 2 & 0.2730 & 0.2740 & 1 \\
\hline 3 & 0.2200 & 0.2200 & 1 & 3 & 0.2300 & 0.2300 & 1 \\
\hline 3 & 0.2200 & 0.2200 & 1 & 3 & 0.2300 & 0.2300 & 1 \\
\hline 3 & 0.2200 & 0.2100 & 1 & 3 & 0.2300 & 0.2300 & 1 \\
\hline 3 & 0.2100 & 0.2200 & 1 & 3 & 0.2300 & 0.2300 & 1 \\
\hline 3 & 0.2200 & 0.2100 & 1 & 3 & 0.2300 & 0.2300 & 1 \\
\hline 3 & 0.2200 & 0.2200 & 1 & 3 & 0.2300 & 0.2200 & 1 \\
\hline 4 & 0.2160 & 0.2150 & 1 & 4 & 0.2160 & 0.2180 & 1 \\
\hline 4 & 0.2170 & 0.2180 & 1 & 4 & 0.2170 & 0.2160 & 1 \\
\hline 4 & 0.2140 & 0.2130 & 1 & 4 & 0.2160 & 0.2190 & 1 \\
\hline 4 & 0.2140 & 0.2150 & 1 & 4 & 0.2170 & 0.2150 & 1 \\
\hline 4 & 0.2190 & 0.2140 & 1 & 4 & 0.2160 & 0.2160 & 1 \\
\hline 4 & 0.2160 & 0.2110 & 1 & 4 & 0.2190 & 0.2160 & 1 \\
\hline 5 & 0.2100 & 0.2200 & 1 & 5 & 0.2110 & 0.2100 & 1 \\
\hline 5 & 0.2200 & 0.2100 & 1 & 5 & 0.2120 & 0.2130 & 1 \\
\hline 5 & 0.2200 & 0.2100 & 1 & 5 & 0.2080 & 0.2120 & 1 \\
\hline 5 & 0.2100 & 0.2100 & 1 & 5 & 0.2130 & 0.2090 & 1 \\
\hline 5 & 0.2200 & 0.2000 & 1 & 5 & 0.2100 & 0.2150 & 1 \\
\hline 5 & 0.2100 & 0.2100 & 1 & 5 & 0.2100 & 0.2110 & 1 \\
\hline 6 & 0.2190 & 0.2140 & 1 & 6 & 0.2200 & 0.2200 & 1 \\
\hline 6 & 0.2140 & 0.2180 & 1 & 6 & 0.2200 & 0.2200 & 1 \\
\hline 6 & 0.2150 & 0.2160 & 1 & 6 & 0.2200 & 0.2200 & 1 \\
\hline 6 & 0.2170 & 0.2190 & 1 & 6 & 0.2200 & 0.2200 & 1 \\
\hline 6 & 0.2200 & 0.2190 & 1 & 6 & 0.2200 & 0.2200 & 1 \\
\hline 6 & 0.2200 & 0.2180 & 1 & 6 & 0.2200 & 0.2200 & 1 \\
\hline 7 & 0.2100 & 0.2100 & 1 & 7 & 0.2200 & 0.2200 & 1 \\
\hline 7 & 0.2100 & 0.2200 & 1 & 7 & 0.2200 & 0.2200 & 1 \\
\hline 7 & 0.2100 & 0.2100 & 1 & 7 & 0.2200 & 0.2200 & 1 \\
\hline 7 & 0.2100 & 0.2100 & 1 & 7 & 0.2200 & 0.2200 & 1 \\
\hline 7 & 0.2100 & 0.2100 & 1 & 7 & 0.2200 & 0.2200 & 1 \\
\hline 7 & 0.2200 & 0.2100 & 1 & 7 & 0.2200 & 0.2200 & 1 \\
\hline 8 & 0.1978 & 0.1916 & 1 & 8 & 0.2170 & 0.2180 & 1 \\
\hline 8 & 0.1970 & 0.1955 & 1 & 8 & 0.2160 & 0.2200 & 1 \\
\hline 8 & 0.1952 & 0.1928 & 1 & 8 & 0.2190 & 0.2190 & 1 \\
\hline 8 & 0.1928 & 0.2011 & 1 & 8 & 0.2210 & 0.2190 & 1 \\
\hline
\end{tabular}




\begin{tabular}{|c|c|c|c|c|c|c|}
\hline 8 & 0.1943 & 0.2003 & 1 & 8 & 0.2200 & 0.2180 \\
\hline 8 & 0.1839 & 0.1874 & 1 & 8 & 0.2210 & 0.2200 \\
\hline 9 & 0.2140 & 0.2150 & 1 & 9 & 0.2100 & 0.2100 \\
\hline 9 & 0.2100 & 0.2120 & 1 & 9 & 0.2200 & 0.2200 \\
\hline 9 & 0.2150 & 0.2170 & 1 & 9 & 0.2200 & 0.2200 \\
\hline 9 & 0.2120 & 0.2110 & 1 & 9 & 0.2200 & 0.2200 \\
\hline 9 & 0.2130 & 0.2140 & 1 & 9 & 0.2200 & 0.2200 \\
\hline 9 & 0.2160 & 0.2160 & 1 & 9 & 0.2200 & 0.2200 \\
\hline 10 & 0.2200 & 0.2100 & 1 & 10 & 0.2090 & 0.2090 \\
\hline 10 & 0.2200 & 0.2200 & 1 & 10 & 0.2080 & 0.2100 \\
\hline 10 & 0.2100 & 0.2200 & 1 & 10 & 0.2100 & 0.2080 \\
\hline 10 & 0.2100 & 0.2200 & 1 & 10 & 0.2110 & 0.2120 \\
\hline 10 & 0.2200 & 0.2200 & 1 & 10 & 0.2120 & 0.2120 \\
\hline 10 & 0.2100 & 0.2200 & 1 & 10 & 0.2110 & 0.2110 \\
\hline 11 & 0.2100 & 0.2110 & 1 & 11 & 0.2120 & 0.2130 \\
\hline 11 & 0.2090 & 0.2080 & 1 & 11 & 0.2140 & 0.2130 \\
\hline 11 & 0.2070 & 0.2070 & 1 & 11 & 0.2140 & 0.2140 \\
\hline 11 & 0.2100 & 0.2110 & 1 & 11 & 0.2130 & 0.2110 \\
\hline 11 & 0.2110 & 0.2100 & 1 & 11 & 0.2120 & 0.2140 \\
\hline 11 & 0.2090 & 0.2100 & 1 & 11 & 0.2130 & 0.2100 \\
\hline 12 & 0.2100 & 0.2100 & 1 & 12 & 0.1160 & 0.1200 \\
\hline 12 & 0.2000 & 0.2000 & 1 & 12 & 0.1190 & 0.1190 \\
\hline 12 & 0.2100 & 0.2100 & 1 & 12 & 0.1190 & 0.1190 \\
\hline 12 & 0.2100 & 0.2100 & 1 & 12 & 0.1180 & 0.1220 \\
\hline 12 & 0.2200 & 0.2000 & 1 & 12 & 0.1180 & 0.1190 \\
\hline 12 & 0.2000 & 0.2100 & 1 & 12 & 0.1210 & 0.1220 \\
\hline 13 & 0.2200 & 0.2200 & 1 & 13 & 0.2100 & 0.2100 \\
\hline 13 & 0.2200 & 0.2200 & 1 & 13 & 0.2100 & 0.2100 \\
\hline 13 & 0.2200 & 0.2200 & 1 & 13 & 0.2000 & 0.2000 \\
\hline 13 & 0.2200 & 0.2200 & 1 & 13 & 0.2100 & 0.2000 \\
\hline 13 & 0.2200 & 0.2200 & 1 & 13 & 0.2100 & 0.2000 \\
\hline 13 & 0.2200 & 0.2200 & 1 & 13 & 0.2000 & 0.2100 \\
\hline 14 & 0.2170 & 0.2200 & 1 & 14 & 0.2210 & 0.2210 \\
\hline 14 & 0.2030 & 0.2370 & 1 & 14 & 0.2200 & 0.2200 \\
\hline 14 & 0.2530 & 0.2460 & 1 & 14 & 0.2210 & 0.2200 \\
\hline 14 & 0.2290 & 0.2230 & 1 & 14 & 0.2200 & 0.2200 \\
\hline 14 & 0.2210 & 0.2080 & 1 & 14 & 0.2200 & 0.2200 \\
\hline 14 & 0.2200 & 0.2260 & 1 & 14 & 0.2190 & 0.2220 \\
\hline 15 & 0.2200 & 0.2200 & 1 & 15 & 0.2220 & 0.2180 \\
\hline 15 & 0.2190 & 0.2210 & 1 & 15 & 0.2150 & 0.2130 \\
\hline 15 & 0.2110 & 0.2130 & 1 & 15 & 0.2150 & 0.2140 \\
\hline 15 & 0.2110 & 0.2110 & 1 & 15 & 0.2890 & 0.2860 \\
\hline 15 & 0.2080 & 0.2170 & 1 & 15 & 0.2860 & 0.2860 \\
\hline 15 & 0.2100 & 0.2190 & 1 & 15 & 0.2760 & 0.2760 \\
\hline 16 & 0.2000 & 0.2000 & 1 & 16 & 0.2134 & 0.2135 \\
\hline 16 & 0.2100 & 0.2000 & 1 & 16 & 0.2148 & 0.2146 \\
\hline 16 & 0.2100 & 0.2000 & 1 & 16 & 0.2156 & 0.2157 \\
\hline 16 & 0.2100 & 0.2100 & 1 & 16 & 0.2115 & 0.2108 \\
\hline 16 & 0.2000 & 0.2000 & 1 & 16 & 0.2147 & 0.2142 \\
\hline
\end{tabular}




\begin{tabular}{|c|c|c|c|c|c|c|}
\hline 16 & 0.2100 & 0.2000 & 1 & 16 & 0.2162 & 0.2172 \\
\hline 17 & 0.2146 & 0.2148 & 1 & 17 & 0.2100 & 0.2100 \\
\hline 17 & 0.2146 & 0.2138 & 1 & 17 & 0.2100 & 0.2100 \\
\hline 17 & 0.2144 & 0.2146 & 1 & 17 & 0.2100 & 0.2100 \\
\hline 17 & 0.2141 & 0.2145 & 1 & 17 & 0.2100 & 0.2100 \\
\hline 17 & 0.2146 & 0.2145 & 1 & 17 & 0.2000 & 0.2100 \\
\hline 17 & 0.2147 & 0.2139 & 1 & 17 & 0.2100 & 0.2100 \\
\hline 18 & 0.2100 & 0.2200 & 1 & 18 & 0.2200 & 0.2195 \\
\hline 18 & 0.2100 & 0.2200 & 1 & 18 & 0.2208 & 0.2205 \\
\hline 18 & 0.2100 & 0.2100 & 1 & 18 & 0.2199 & 0.2196 \\
\hline 18 & 0.2100 & 0.2100 & 1 & 18 & 0.2194 & 0.2195 \\
\hline 18 & 0.2100 & 0.2100 & 1 & 18 & 0.2196 & 0.2193 \\
\hline 18 & 0.2100 & 0.2100 & 1 & 18 & 0.2197 & 0.2197 \\
\hline 19 & 0.2100 & 0.2170 & 1 & 19 & 0.2200 & 0.2200 \\
\hline 19 & 0.2110 & 0.2140 & 1 & 19 & 0.2200 & 0.2200 \\
\hline 19 & 0.2090 & 0.2040 & 1 & 19 & 0.2200 & 0.2200 \\
\hline 19 & 0.2100 & 0.2020 & 1 & 19 & 0.2200 & 0.2200 \\
\hline 19 & 0.2160 & 0.2160 & 1 & 19 & 0.2200 & 0.2200 \\
\hline 19 & 0.2150 & 0.2120 & 1 & 19 & 0.2200 & 0.2200 \\
\hline 1 & 0.0880 & 0.0940 & 2 & 20 & 0.2150 & 0.2170 \\
\hline 1 & 0.0920 & 0.0910 & 2 & 20 & 0.2180 & 0.2160 \\
\hline 1 & 0.0990 & 0.0970 & 2 & 20 & 0.2160 & 0.2170 \\
\hline 1 & 0.0920 & 0.0970 & 2 & 20 & 0.2170 & 0.2180 \\
\hline 1 & 0.0950 & 0.0920 & 2 & 20 & 0.2170 & 0.2160 \\
\hline 1 & 0.0920 & 0.0970 & 2 & 20 & 0.2080 & 0.2070 \\
\hline 2 & 0.0918 & 0.0928 & 2 & 21 & 0.2020 & 0.2040 \\
\hline 2 & 0.0908 & 0.0918 & 2 & 21 & 0.2190 & 0.2190 \\
\hline 2 & 0.0928 & 0.0918 & 2 & 21 & 0.2100 & 0.2090 \\
\hline 2 & 0.0938 & 0.0929 & 2 & 21 & 0.2310 & 0.2260 \\
\hline 2 & 0.0929 & 0.0938 & 2 & 21 & 0.2070 & 0.2090 \\
\hline 2 & 0.0919 & 0.0938 & 2 & 21 & 0.2170 & 0.2190 \\
\hline 3 & 0.0900 & 0.0900 & 2 & 1 & 0.0900 & 0.0900 \\
\hline 3 & 0.0900 & 0.0900 & 2 & 1 & 0.0900 & 0.0900 \\
\hline 3 & 0.0900 & 0.0900 & 2 & 1 & 0.0900 & 0.0900 \\
\hline 3 & 0.0900 & 0.0900 & 2 & 1 & 0.1000 & 0.1000 \\
\hline 3 & 0.0900 & 0.0900 & 2 & 1 & 0.0900 & 0.1000 \\
\hline 3 & 0.0900 & 0.0900 & 2 & 1 & 0.0900 & 0.0900 \\
\hline 4 & 0.0890 & 0.0920 & 2 & 2 & 0.1230 & 0.1250 \\
\hline 4 & 0.0940 & 0.0930 & 2 & 2 & 0.1280 & 0.1240 \\
\hline 4 & 0.0930 & 0.0930 & 2 & 2 & 0.1250 & 0.1280 \\
\hline 4 & 0.0920 & 0.0890 & 2 & 2 & 0.1230 & 0.1240 \\
\hline 4 & 0.0920 & 0.0910 & 2 & 2 & 0.1280 & 0.1250 \\
\hline 4 & 0.0920 & 0.0900 & 2 & 2 & 0.1190 & 0.1250 \\
\hline 5 & 0.1000 & 0.1010 & 2 & 3 & 0.1100 & 0.1000 \\
\hline 5 & 0.0990 & 0.1010 & 2 & 3 & 0.1000 & 0.1000 \\
\hline 5 & 0.0980 & 0.1010 & 2 & 3 & 0.1000 & 0.0900 \\
\hline 5 & 0.0890 & 0.0950 & 2 & 3 & 0.1100 & 0.1000 \\
\hline 5 & 0.1010 & 0.1010 & 2 & 3 & 0.1100 & 0.1000 \\
\hline 5 & 0.1000 & 0.1000 & 2 & 3 & 0.1000 & 0.1000 \\
\hline
\end{tabular}




\begin{tabular}{|c|c|c|c|c|c|c|}
\hline 6 & 0.0910 & 0.0950 & 2 & 4 & 0.0910 & 0.0900 \\
\hline 6 & 0.0960 & 0.0910 & 2 & 4 & 0.0920 & 0.0920 \\
\hline 6 & 0.0910 & 0.0930 & 2 & 4 & 0.0870 & 0.0870 \\
\hline 6 & 0.0940 & 0.0920 & 2 & 4 & 0.0890 & 0.0890 \\
\hline 6 & 0.0920 & 0.0910 & 2 & 4 & 0.0900 & 0.0890 \\
\hline 6 & 0.0940 & 0.0930 & 2 & 4 & 0.0880 & 0.0900 \\
\hline 7 & 0.0900 & 0.0900 & 2 & 5 & 0.0890 & 0.0880 \\
\hline 7 & 0.0900 & 0.0900 & 2 & 5 & 0.0850 & 0.0860 \\
\hline 7 & 0.0900 & 0.0900 & 2 & 5 & 0.0850 & 0.0860 \\
\hline 7 & 0.0900 & 0.0900 & 2 & 5 & 0.0890 & 0.0870 \\
\hline 7 & 0.0900 & 0.0900 & 2 & 5 & 0.0880 & 0.0900 \\
\hline 7 & 0.0900 & 0.0900 & 2 & 5 & 0.0860 & 0.0850 \\
\hline 8 & 0.0918 & 0.0912 & 2 & 6 & 0.1000 & 0.1000 \\
\hline 8 & 0.0924 & 0.0976 & 2 & 6 & 0.1000 & 0.1000 \\
\hline 8 & 0.0930 & 0.0928 & 2 & 6 & 0.1000 & 0.1000 \\
\hline 8 & 0.0898 & 0.0927 & 2 & 6 & 0.1000 & 0.1000 \\
\hline 8 & 0.0957 & 0.0947 & 2 & 6 & 0.1000 & 0.1000 \\
\hline 8 & 0.0949 & 0.0912 & 2 & 6 & 0.1000 & 0.1000 \\
\hline 9 & 0.0910 & 0.0950 & 2 & 7 & 0.0900 & 0.0900 \\
\hline 9 & 0.0950 & 0.0900 & 2 & 7 & 0.0900 & 0.0900 \\
\hline 9 & 0.0930 & 0.0920 & 2 & 7 & 0.0900 & 0.0900 \\
\hline 9 & 0.0930 & 0.0920 & 2 & 7 & 0.0900 & 0.0900 \\
\hline 9 & 0.0920 & 0.0950 & 2 & 7 & 0.0900 & 0.0900 \\
\hline 9 & 0.0910 & 0.0960 & 2 & 7 & 0.0900 & 0.0900 \\
\hline 10 & 0.0900 & 0.0900 & 2 & 8 & 0.0890 & 0.0920 \\
\hline 10 & 0.0900 & 0.0900 & 2 & 8 & 0.0910 & 0.0920 \\
\hline 10 & 0.0900 & 0.0900 & 2 & 8 & 0.0910 & 0.0920 \\
\hline 10 & 0.0900 & 0.0900 & 2 & 8 & 0.0890 & 0.0900 \\
\hline 10 & 0.0900 & 0.0900 & 2 & 8 & 0.0920 & 0.0920 \\
\hline 10 & 0.0900 & 0.0900 & 2 & 8 & 0.0900 & 0.0910 \\
\hline 11 & 0.0910 & 0.0910 & 2 & 9 & 0.1000 & 0.1000 \\
\hline 11 & 0.0900 & 0.0900 & 2 & 9 & 0.1100 & 0.1000 \\
\hline 11 & 0.0900 & 0.0900 & 2 & 9 & 0.1000 & 0.1100 \\
\hline 11 & 0.0900 & 0.0900 & 2 & 9 & 0.1000 & 0.1000 \\
\hline 11 & 0.0900 & 0.0910 & 2 & 9 & 0.1000 & 0.1000 \\
\hline 11 & 0.0890 & 0.0900 & 2 & 9 & 0.1000 & 0.1000 \\
\hline 12 & 0.0900 & 0.0900 & 2 & 10 & 0.0910 & 0.0920 \\
\hline 12 & 0.0900 & 0.0900 & 2 & 10 & 0.0900 & 0.0900 \\
\hline 12 & 0.0900 & 0.0900 & 2 & 10 & 0.0900 & 0.0900 \\
\hline 12 & 0.0900 & 0.0900 & 2 & 10 & 0.0940 & 0.0950 \\
\hline 12 & 0.0900 & 0.0900 & 2 & 10 & 0.0930 & 0.0940 \\
\hline 12 & 0.0900 & 0.0900 & 2 & 10 & 0.0920 & 0.0930 \\
\hline 13 & 0.1000 & 0.0900 & 2 & 11 & 0.0930 & 0.0920 \\
\hline 13 & 0.0900 & 0.0900 & 2 & 11 & 0.0910 & 0.0920 \\
\hline 13 & 0.0900 & 0.0900 & 2 & 11 & 0.0900 & 0.0900 \\
\hline 13 & 0.0900 & 0.1000 & 2 & 11 & 0.0900 & 0.0880 \\
\hline 13 & 0.0900 & 0.1000 & 2 & 11 & 0.0910 & 0.0910 \\
\hline 13 & 0.0900 & 0.0900 & 2 & 11 & 0.0910 & 0.0900 \\
\hline 14 & 0.0960 & 0.0820 & 2 & 12 & 0.0700 & 0.0690 \\
\hline
\end{tabular}




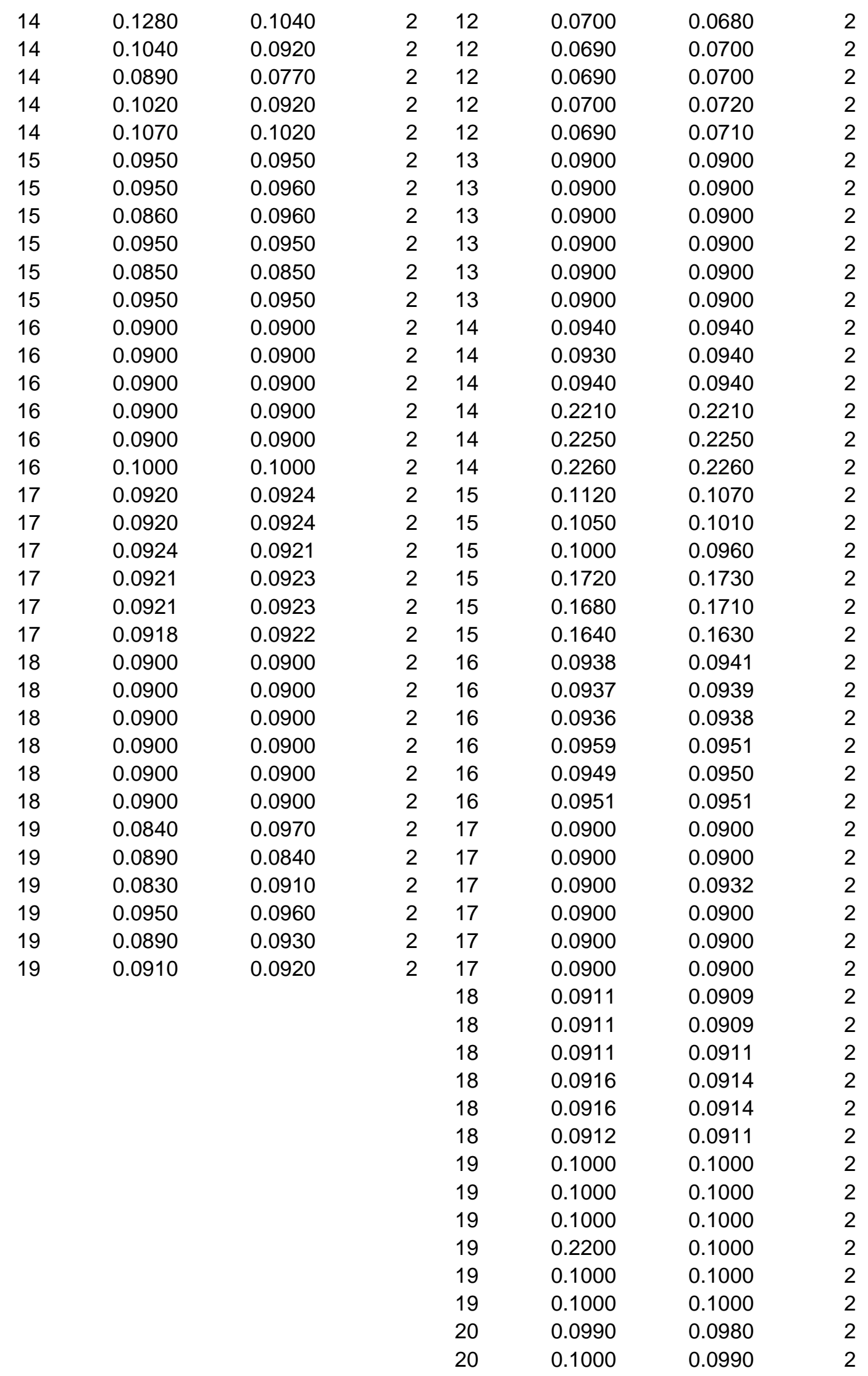




$\begin{array}{llll}20 & 0.0910 & 0.0900 & 2 \\ 20 & 0.0920 & 0.0910 & 2 \\ 20 & 0.0960 & 0.0970 & 2 \\ 20 & 0.0980 & 0.0980 & 2 \\ 21 & 0.0820 & 0.0810 & 2 \\ 21 & 0.0940 & 0.0860 & 2 \\ 21 & 0.0960 & 0.0930 & 2 \\ 21 & 0.0990 & 0.0970 & 2 \\ 21 & 0.0930 & 0.0980 & 2 \\ 21 & 0.0820 & 0.0870 & 2\end{array}$




\begin{tabular}{|c|c|c|c|c|}
\hline Lab & Method & Duplicate 1 & Duplicate 2 & Cement \\
\hline 1 & glass & 0.005 & 0.006 & 1 \\
\hline 1 & glass & 0.003 & 0.005 & 1 \\
\hline 1 & glass & 0.004 & 0.004 & 1 \\
\hline 1 & glass & 0.004 & 0.003 & 1 \\
\hline 1 & glass & 0.002 & 0.003 & 1 \\
\hline 1 & glass & 0.003 & 0.004 & 1 \\
\hline 1 & glass & 0.056 & 0.059 & 2 \\
\hline 1 & glass & 0.067 & 0.067 & 2 \\
\hline 1 & glass & 0.055 & 0.054 & 2 \\
\hline 1 & glass & 0.054 & 0.052 & 2 \\
\hline 1 & glass & 0.057 & 0.059 & 2 \\
\hline 1 & glass & 0.058 & 0.058 & 2 \\
\hline 2 & glass & 0.0123 & 0.0136 & 1 \\
\hline 2 & glass & 0.0117 & 0.0117 & 1 \\
\hline 2 & glass & 0.0111 & 0.0114 & 1 \\
\hline 2 & glass & 0.011 & 0.0117 & 1 \\
\hline 2 & glass & 0.0127 & 0.0108 & 1 \\
\hline 2 & glass & 0.0125 & 0.0115 & 1 \\
\hline 2 & glass & 0.098 & 0.0976 & 2 \\
\hline 2 & glass & 0.0924 & 0.0914 & 2 \\
\hline 2 & glass & 0.094 & 0.0951 & 2 \\
\hline 2 & glass & 0.0922 & 0.0943 & 2 \\
\hline 2 & glass & 0.082 & 0.0819 & 2 \\
\hline 2 & glass & 0.0915 & 0.0918 & 2 \\
\hline 3 & glass & 0.0037 & 0.0038 & 1 \\
\hline 3 & glass & 0.0035 & 0.0037 & 1 \\
\hline 3 & glass & 0.0039 & 0.0034 & 1 \\
\hline 3 & glass & 0.0035 & 0.0034 & 1 \\
\hline 3 & glass & 0.0034 & 0.0034 & 1 \\
\hline 3 & glass & 0.0032 & 0.0034 & 1 \\
\hline 3 & glass & 0.0067 & 0.0076 & 2 \\
\hline 3 & glass & 0.0092 & 0.0089 & 2 \\
\hline 3 & glass & 0.008 & 0.0084 & 2 \\
\hline 3 & glass & 0.0077 & 0.0082 & 2 \\
\hline 3 & glass & 0.0081 & 0.0087 & 2 \\
\hline 3 & glass & 0.006 & 0.0064 & 2 \\
\hline 4 & powder & 0.017 & 0.018 & 1 \\
\hline 4 & powder & 0.017 & 0.017 & 1 \\
\hline 4 & powder & 0.017 & 0.017 & 1 \\
\hline 4 & powder & 0.019 & 0.02 & 1 \\
\hline 4 & powder & 0.017 & 0.017 & 1 \\
\hline 4 & powder & 0.017 & 0.018 & 1 \\
\hline 4 & powder & 0.157 & 0.157 & 2 \\
\hline 4 & powder & 0.158 & 0.159 & 2 \\
\hline 4 & powder & 0.159 & 0.159 & 2 \\
\hline 4 & powder & 0.154 & 0.155 & 2 \\
\hline 4 & powder & 0.156 & 0.159 & 2 \\
\hline
\end{tabular}




\begin{tabular}{|c|c|c|c|}
\hline 4 & powder & 0.157 & 0.158 \\
\hline 6 & powder & 0.0073 & 0.0079 \\
\hline 6 & powder & 0.008 & 0.0083 \\
\hline 6 & powder & 0.0081 & 0.0082 \\
\hline 6 & powder & 0.0082 & 0.0088 \\
\hline 6 & powder & 0.0091 & 0.0099 \\
\hline 6 & powder & 0.0091 & 0.0094 \\
\hline 6 & powder & 0.0854 & 0.0852 \\
\hline 6 & powder & 0.0804 & 0.0813 \\
\hline 6 & powder & 0.0812 & 0.082 \\
\hline 6 & powder & 0.0831 & 0.0853 \\
\hline 6 & powder & 0.0837 & 0.0857 \\
\hline 6 & powder & 0.0815 & 0.0828 \\
\hline 7 & powder & 0.0173 & 0.0176 \\
\hline 7 & powder & 0.0174 & 0.0181 \\
\hline 7 & powder & 0.0172 & 0.0204 \\
\hline 7 & powder & 0.0126 & 0.0127 \\
\hline 7 & powder & 0.0132 & 0.0131 \\
\hline 7 & powder & 0.0134 & 0.0135 \\
\hline 7 & powder & 0.2331 & 0.233 \\
\hline 7 & powder & 0.237 & 0.238 \\
\hline 7 & powder & 0.2375 & 0.2378 \\
\hline 7 & powder & 0.1861 & 0.1869 \\
\hline 7 & powder & 0.1867 & 0.188 \\
\hline 7 & powder & 0.1896 & 0.1867 \\
\hline 8 & powder & 0.004 & 0.004 \\
\hline 8 & powder & 0.004 & 0.004 \\
\hline 8 & powder & 0.004 & 0.004 \\
\hline 8 & powder & 0.004 & 0.004 \\
\hline 8 & powder & 0.004 & 0.004 \\
\hline 8 & powder & 0.004 & 0.004 \\
\hline 8 & powder & 0.0055 & 0.0055 \\
\hline 8 & powder & 0.0055 & 0.0055 \\
\hline 8 & powder & 0.0055 & 0.0055 \\
\hline 8 & powder & 0.0055 & 0.0055 \\
\hline 8 & powder & 0.0055 & 0.0055 \\
\hline 8 & powder & 0.0055 & 0.0055 \\
\hline 9 & powder & 0.0093 & 0.0109 \\
\hline 9 & powder & 0.0099 & 0.0096 \\
\hline 9 & powder & 0.0089 & 0.0095 \\
\hline 9 & powder & 0.0095 & 0.0099 \\
\hline 9 & powder & 0.0097 & 0.0097 \\
\hline 9 & powder & 0.0099 & 0.0099 \\
\hline 9 & powder & 0.0723 & 0.0727 \\
\hline 9 & powder & 0.0734 & 0.0742 \\
\hline 9 & powder & 0.0743 & 0.0746 \\
\hline 9 & powder & 0.0738 & 0.0744 \\
\hline 9 & powder & 0.073 & 0.0741 \\
\hline 9 & powder & 0.0735 & 0.0749 \\
\hline
\end{tabular}




\section{Acknowledgements}

We wish to recognize LeRoy Jacobs, who provided the enthusiasm and his talents to the ASTM C1.23 task group to get this project underway. The time, efforts of all of the participants in this trial program and Robin Haupt of the Cement and Concrete Reference laboratory for sample distribution and collecting data are acknoledged. The comments and suggestions of reviewers Clarissa Ferraris, Kenneth Snyder and Don Broton are gratefully acknowledged. This project was supported by the Early-Age Performance of Concrete project within the Sustainable Engineering Materials program at NIST.

\section{References}

${ }^{1}$ Data Considered by Committee C-1 of the American Society for Testing Materials in Preparing the Standard Specifications and Tests for Portland Cement (serial Designation C 9-17), Committee C-1, American Society for Testing Materials, Philadelphia, PA, July 1919.

${ }^{2}$ C114-11b, "Standard Test Method for Chemical Analysis of Hydraulic Cement," Annual Book of ASTM Standards, Vol. 4.01, ASTM International, West Conshohocken, PA. ${ }^{3}$ J.A. Forrester, T.P. Lees, and A.E. Moore, "The Precision of Standard Cement Analysis nd its Effect on the Calculated Compound Composition," S.C.I. Monograph No. 18, The Analysis of Calcareous Materials, 447-451 (1964)

${ }^{4}$ H.G. Midgley, "Compound calculation in the phases in Portland cement clinker," Cement Technology, Vol. 1, No. 3, 1970, pp. 1-5.

5 A.M. Harrisson, H.F.W. Taylor and N.B. Winter, "Electron-Optical Analysis of the Phases in a Portland Cement Clinker, with some Observations on the Calculation of Quantitative Phase Composition”, Cement and Concrete Research, Vol. 15, pp. 775-780, 1985.

${ }^{6}$ L.P. Aldridge and R.P. Eardley, "Effects of analytical errors on the Bogue calculation of compound composition," Cement Technology, 4, 1973, pp. 177-182.

${ }^{7}$ L.P. Aldridge, "Errors in the analysis of cement," Cement Technology, 7, 1976, pp. 8-11

${ }^{8}$ P.E. Stutzman, and D.S. Lane, "Effects of Analytical Precision on Bogue Calculations of Potential Portland Cement Composition,” Journal of ASTM International, Vol. 7, No. 6, paper ID JAI102697

${ }^{9}$ EN 196-2, Methods of Testing Cement - Part 2: Chemical analysis of cement,

${ }^{10}$ NCHRP 139, "Precision Estimates for AASHTO Test Method T 105, Determined Using CCRL Proficiency Sample Data”. NCHRP Project 09-26A, June 2009,

http://onlinepubs.trb.org/onlinepubs/nchrp/nchrp_w139.pdf

${ }_{11}$ R. Haupt, personal communication

12 ASTM E 691 "Standard Practice for Conducting an Interlaboratory Study to Determine the Precision of a Test Method," Annual Book of ASTM Standards, Vol. 14.02, ASTM International, West Conshohocken, PA.

${ }^{13}$ G.J. Hahn and W.Q. Meeker, Statistical Intervals: A Guide for Practitioners, John Wiley and Sons, New York, 1991. 